\title{
IDENTIFICATION OF A MENINGOCOCCAL ADHESIN THAT INHIBITS EPITHELIAL CELL MIGRATION
}

by

\author{
Gabrielle Alice Greig
}

\begin{abstract}
A thesis
submitted to the Victoria University of Wellington in fulfilment of the requirements for the degree of Doctor of Philosophy in Molecular Microbiology
\end{abstract}





\section{Primary Supervisor}

Dr. Joanna MacKichan

School of Biological Sciences

Victoria University of Wellington

\section{ESR Supervisor}

Dr. Philip Carter

Principal Scientist, Kenepuru Science Centre,

Institute of Environmental Science and Research 



\section{Abstract}

Neisseria meningitidis virulence is polygenic, therefore comparing many genomes may not yield strictly disease-associated virulence factors. An alternative approach is comparing closely related isolates, such as those from household contacts. Disease isolates have been shown to inhibit epithelial cell wound repair, while many carriage isolates do not. In this study, bacteria collected from disease patients and healthy household contacts were compared to identify the meningococcal factor responsible for wound repair inhibition and investigate how it contributes to invasive disease. Host cell wound repair inhibition was compared between diseaseassociated meningococcal isolate, NZ97/052, and isolates NZCM111 and NZCM112, from asymptomatic household contacts. Migrating bronchial airway cells were infected with meningococcal isolates and wound closure was evaluated by microscopy. NZ97/052 and NZCM111 both inhibited wound repair, whereas NZCM112 did not. To investigate if this was due to bacterial consumption of an important nutrient, infected cells were supplemented with nutrients known to be important for meningococcal growth and cell migration. Iron supplementation resulted in carriage associated isolates gaining the ability to inhibit wound repair. Genome and transcriptome comparisons were completed between NZ97/052 and NZCM112, which differ in wound repair inhibition. This analysis identified a frameshift mutation in NZCM112 in the haptoglobin-haemoglobin utilization gene, $h p u A$, which caused complete loss of expression. The hpuA gene was deleted by allelic replacement from NZ97/052, nullifying its ability to inhibit host cell migration. Furthermore, bacterial association and fluorescence microscopy assays suggested that HpuA contributes to meningococcal attachment to bronchial epithelial cells, as the hpuA mutant had significantly lower cell association. Heterologous expression of HpuA in E. coli resulted in higher levels of cell association, indicating that HpuA is sufficient to mediate bacterial adhesion to human bronchial epithelial cells. This work reveals novel roles for HpuA as a meningococcal adhesin and a bacterial factor that inhibits host cell migration. 


\section{Acknowledgements}

First, I would like to express my deepest gratitude to my supervisor Dr. Joanna Mackichan for taking me on as a student. Your guidance, patience, and thoughtful encouragement made this research possible. I am extremely grateful for all you have taught me. Thank you to my ESR supervisor, Dr. Philip Carter, for your sage advice and faith in my abilities.

I would like to acknowledge ESR Kenepuru Science Centre for the use of their lab and office space. To everyone in the invasive pathogens lab; Heather, Julie, Zoe, Michelle, and Audrey, thank you for creating such an awesome and welcoming lab environment. Special thanks to Dr. Una Ren for your expertise and always being willing to give advice and share protocols. Your generosity of knowledge and help over the years has been utterly invaluable.

Thank you to my friends and family for helping me survive the stress of a PhD. Mum, thank you for the emotional support and for always believing that I could. Your pride in me and my research has always meant the world. Dad, thanks for always trying to understand the finer details of this project, and for diligently reading this entire thesis. You know more about PCR than you have any business knowing. Thanks to Max for being a great writing buddy.

Finally, thank you to my incredible fiancé Nick. You're my rock, and your love and support over the years has never wavered. Thank you for believing in me, even when I didn't. 


\section{Table of Contents}

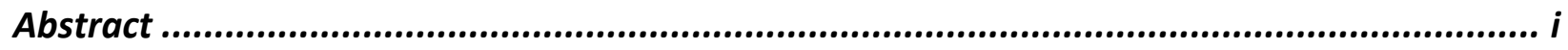





List of Figures













1.1.3 Biology of N. meningitidis and bacterial surface structures ................................... 5

1.1.4 Iron availability in the human host and bacterial iron acquisition ............................ 6



1.1.5.1 Progression of invasive meningococcal disease ............................................. 9





1.1.7.1 New Zealand and Meningococcal disease ....................................................16



1.1.8 Prevention and treatment of N. meningitidis ..................................................... 18

1.2 Epithelial cell wound repair and invasive meningococcal disease .................................20



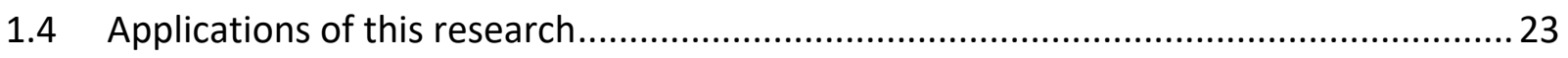

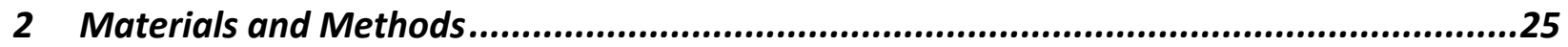








2.1.1.1 Growth and storage of $N$. meningitidis................................................... 27

2.1.1.2 Making frozen stocks of N. meningitidis................................................... 27

2.1.1.3 N. meningitidis growth from frozen stock ............................................. 27





2.1.3 Antibiotic for selective bacterial growth .................................................... 28



2.2.1 Recovery of 16HBE cells from frozen stocks ............................................... 29

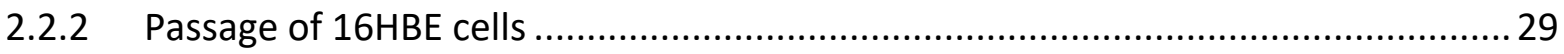





2.2.4.1 Culturing 16HBE cells onto chamber slides for fluorescence microscopy ..........30

2.2.4.2 Culturing 16HBE cells onto Oris cell migration plates .................................. 30

2.2.4.3 Culture of polarised 16 HBE cells .............................................................. 31






























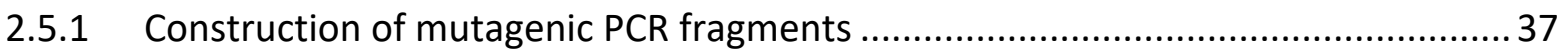

2.5.2 Natural transformation for mutagenesis of $N$. meningitidis. ............................... 38

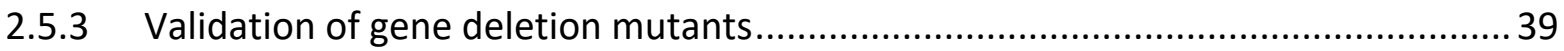

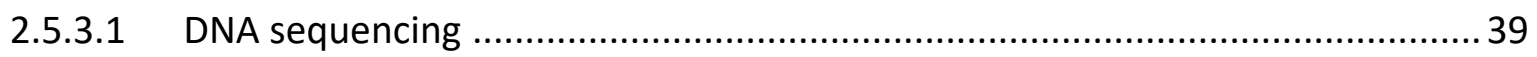





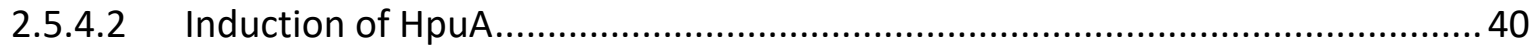

2.5.5 Confirmation of HpuA expression by protein gel analysis ................................ 41



2.5.5.2 Preparation of reagents for SDS-PAGE ................................................... 41



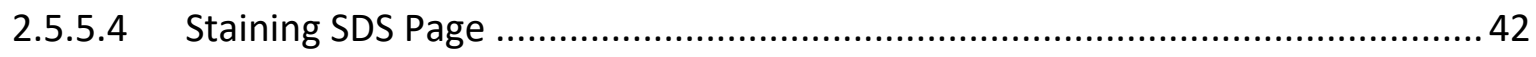

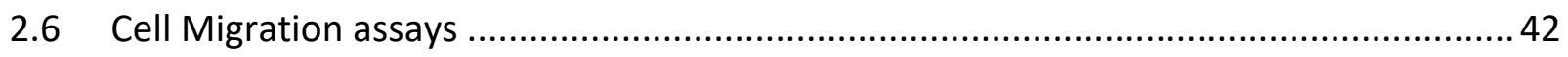



2.6.2 In vitro wound repair scratch assay ........................................................... 43

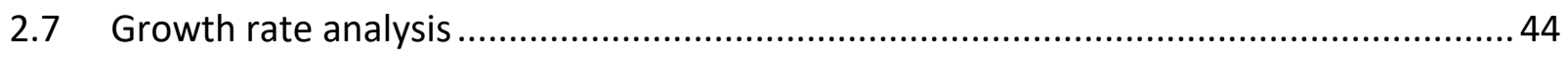



2.7.2 Counting viable colony forming units...................................................... 44

2.8 Cell association and invasion assays ......................................................... 45

2.8.1 Cell-association assay with polarised $16 \mathrm{HBE}$ cells.......................................... 45



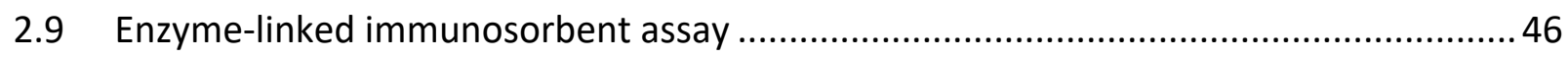

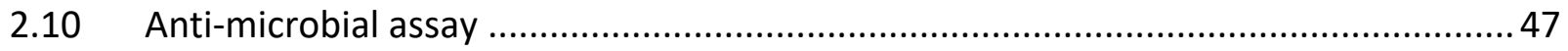

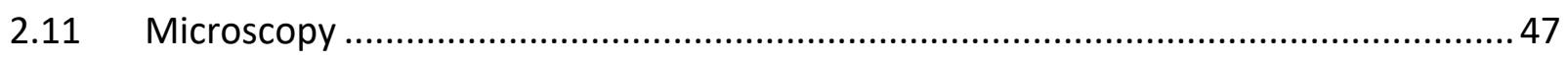

2.11.1 Preparation of paraformaldehyde for fixation of cells .................................. 47

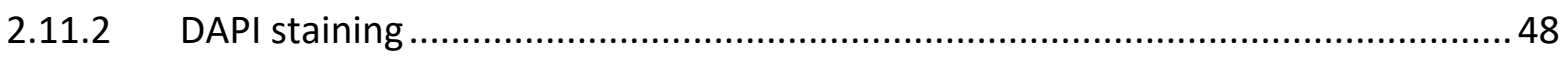



2.11.4 Digital image acquisition of stained chamber slides ...................................... 49 


\section{Investigation of phenotypic differences between NZ97/052 household meningococcal}

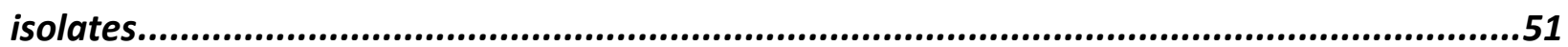

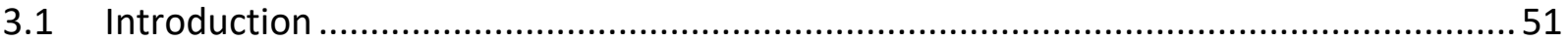





3.2.1 Development of an epithelial cell migration assay ..............................................53

3.2.2 Related N. meningitidis isolates differ in their ability to inhibit cell re-

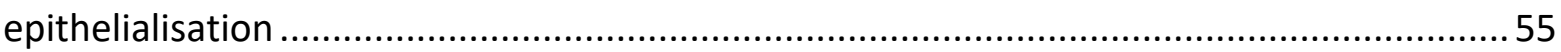

3.2.3 NZCM111 and NZ97/052 grow more efficiently than NZCM112 when cultured with

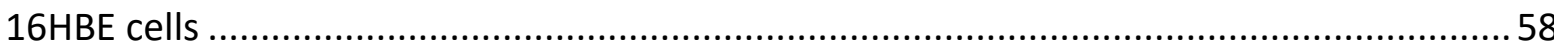

3.2.4 Increasing the multiplicity of infection of $N$. meningitidis does not impact host cell

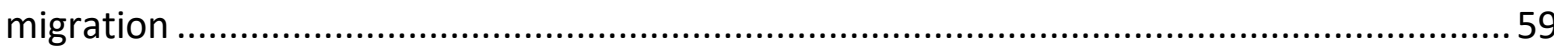

3.2.5 NZ97/052 has greater association to 16HBE cells than NZCM112 .........................61

3.2.6 NZ97/052 isolate has higher association to polarised human respiratory epithelial





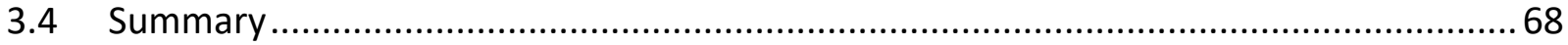

4 Investigation of N. meningitidis metabolism and host cell migration..............................69

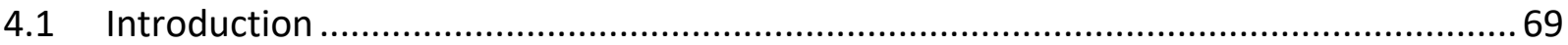

4.1.1 Transition metals, amino acids, and nutritional immunity .................................... 70

4.1.2 Aims of this chapter ..........................................................................................

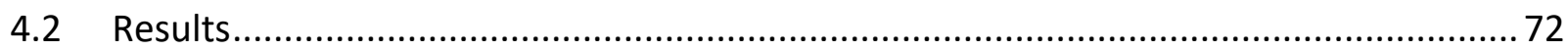

4.2.1 Supplementation with non-essential amino acids or zinc does not affect wound repair inhibition

4.2.2 Iron supplementation increases wound repair inhibition by carriage-associated isolate NZCM112 
4.2.3 Inhibition of re-epithelialisation by other 'carriage-associated' isolates increases in



4.2.4 NZCM112 and NZ97/052 differ in growth rate measured over a time course ........79

4.2.5 Supplementation with iron, zinc, or non-essential amino acids does not affect bacterial growth in NZ97/052 household in the presence of 16 HBE cells ......................... 82

4.2.6 Association of NZCM112 with 16HBE cells increases in the presence of iron..........85

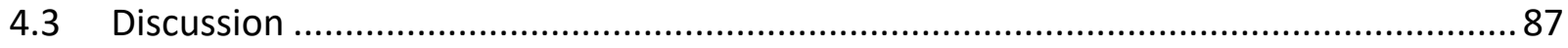

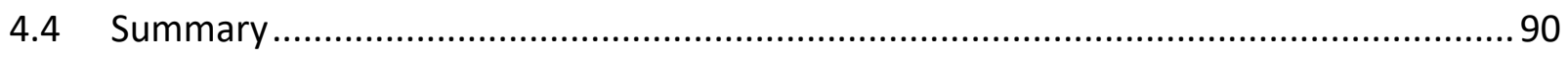

\section{Identification of the meningococcal factor responsible for respiratory epithelial cell} wound repair inhibition .............................................................................91

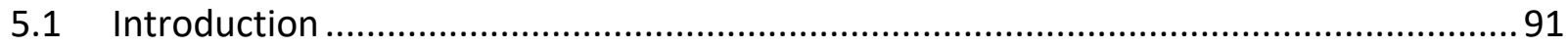





5.2.1 Genome comparisons between isolates in the NZ97/052 household ..................92

5.2.2 Transcriptome comparison of isolates in the NZ97/052 household ................... 100

5.2.3 Candidate genes selected for deletion from NZ97/052 ................................... 109

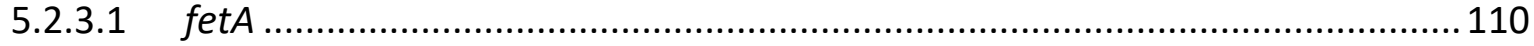



5.2.3.3

5.2.4 Neisseria meningitidis mutagenesis ......................................................... 113

5.2.4.1 PCR amplification of regions of homology flanking candidate genes............. 116

5.2.4.2 PCR amplification of $a p h A 3$ gene ..................................................... 117

5.2.4.3 Construction of fusion products and mutagenesis .................................. 118

5.2.4.4 Selection and validation of gene deletion mutants ................................. 120

5.2.5 Effect of FetA and Fur on wound repair .................................................. 121

5.2.6 Effect of HpuA and HpuB on wound repair ............................................ 122

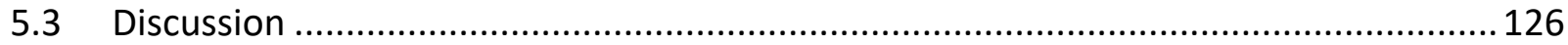


6.1 Introduction 133

6.1.1 HpuAB - A Neisseria haptoglobin-haemoglobin receptor 134

6.1.2 Aims of the chapter .136

6.2 Results

6.2.1 Human haptoglobin supplementation has a slight impact on wound repair inhibition by NZ97/052 and NZCM112.

6.2.2 Haptoglobin levels remain constant in bacteria-host cell co-culture. 139

6.2.3 Fluorescence microscopy identified higher numbers of cell association of NZ97/052 than NZCM112 and NZ97/052 $\triangle h p u A$ 142

6.2.4 Haptoglobin does not exhibit an antimicrobial effect on N. meningitidis isolates 144

6.2.5 Isolate NZCM112, but not NZ97/052 $\triangle h p u A$, grows at a slower rate than NZ97/052. 146

6.2.5.1 NZCM112 has reduced growth in the presence of respiratory epithelial cells, relative to NZ97/052 and NZ97/052 $\triangle$ hpuA. 148

6.2.6 HpuA increases association of bacteria with human respiratory epithelial cells...150

6.2.7 NZ97/052 displays higher levels of invasion of human respiratory epithelial cells than NZCM112 and NZ97/052 $\triangle h p u A$

6.2.7.1 Fluorescence microscopy analysis confirms reduced bacterial attachment of NZCM112 and NZ97/052 $\triangle h p u A$ to $16 \mathrm{HBE}$ cells

6.2.8 Heterologous expression of HpuA in E. coli 157

6.2.8.1 Expression of HpuA by E. coli did not adversely impact bacterial growth....... 159 6.2.8.2 E. coli expressing HpuA has increased association with 16HBE respiratory epithelial cells. 161

6.2.9 Protein sequence alignment of HpuA homologues 163

6.3 Discussion 167

6.4 Summary 173 






7.3 Limitations of research using $N$. meningitidis ................................................. 180



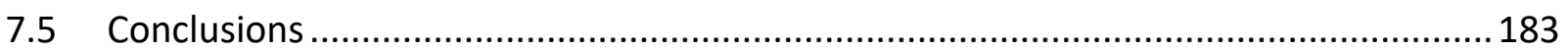

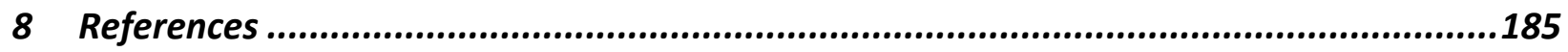

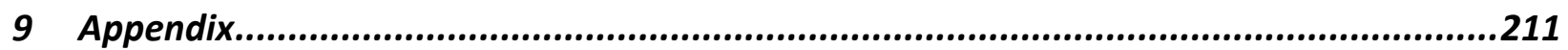



9.2 Expression levels of differentially regulated genes.............................................. 212

9.3 Genes not involved in inhibition of epithelial cell migration................................. 214

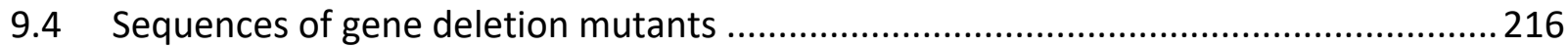

9.5 Sulfur uptake pathway in N. meningitidis ......................................................... 219



9.5.2 Hydrogen peroxide sensitivity assay protocol................................................ 219

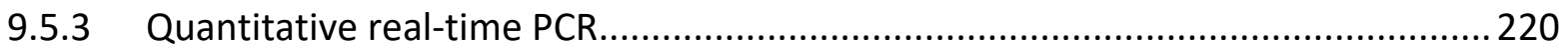






\section{List of Figures}

Figure 1.1 Stages of pathogenesis for $N$. meningitidis. .................................................... 10

Figure 1.2 Common features of the meningococcal outer membrane................................... 12

Figure 1.3 Example of Serogroup-Serotype-Serosubtype classification of N. meningitidis. ........ 13

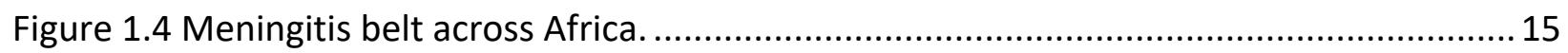

Figure 1.5 Meningococcal disease notifications in New Zealand by group by quarter by year,

Figure 3.1. Oris cell migration assay protocol overview. .54

Figure 3.2. Representative photographs from Oris cell migration assay are shown, including the initial wound area (wound). .56

Figure 3.3. Disease isolate NZ97/052 and carriage isolate NZCM111 inhibit airway cell migration, but NZCM112 does not. .57

Figure 3.4. NZ97/052 grow more efficiently than NZCM112 in the presence of $16 \mathrm{HBE}$ cells......59 Figure 3.5. The inhibition of cell migration did not vary significantly with increasing multiplicity of infection of meningococci. 60

Figure 3.6. NZ97/052 has higher levels of association to 16HBE cells compared to NZCM112...63

Figure 3.7. Transwell system showing basal and apical chambers .64

Figure 3.8. NZ97/052 had significantly higher association with polarised $16 \mathrm{HBE}$ cells, relative to NZCM112, on the apical surface in a Transwell system. .65

Figure 4.1. Supplementation of the cell migration assay with zinc and non-essential amino acids (NEAA) did not affect cell migration, either with or without meningococci. .74

Figure 4.2. Addition of iron ( $>1 \mu \mathrm{M})$ resulted in NZCM112-mediated cell migration inhibition.. 77 Figure 4.3. Addition of $20 \mu \mathrm{M} \mathrm{FeSO}_{4}$ to the cell culture medium resulted in the ability of multiple carriage isolates to inhibit wound repair.

Figure 4.4. The growth rate of NZ97/052 is higher than NZCM112 and iron sulfate supplementation does not impact final concentration reached.

Figure 4.5. Nutrient supplementation did not significantly alter bacterial growth in the presence of host cells. NZ97/052 grew to higher numbers than NZCM112. .84 
Figure 4.6. Iron supplementation increases association to and invasion of $16 \mathrm{HBE}$ cells by NZCM112.

Figure 5.1.Nucleotide sequence alignment of hpuA from NZ97/052 and NZCM112. .99

Figure 5.2. Protein sequence alignment of HpuA from NZ97/052 and NZCM112. 100

Figure 5.3. Volcano plot of differentially expressed genes between isolates NZ97/052 and NZCM112.

Figure 5.4. Forty-eight of the most highly differentially expressed transcripts between isolates NZ97/052 and NZCM112. 102

Figure 5.5. Predicted cellular location and functional classification of differentially expressed genes according to the UniProt database. 109

Figure 5.6. Schematic representation of HpuAB iron acquisition system in N. meningitidis.....113

Figure 5.7. Overview of gene replacement with antibiotic resistant cassette in Neisseria meningitidis. 115

Figure 5.8. Amplification of DNA flanking candidate genes, visualised on $2 \%$ agarose gel.

Figure 5.9. PCR of aphA3 gene, amplified from pUC19 Km, visualized on $2 \%$ agarose gel. 118

Figure 5.10. PCR to form recombinant gene fusion products visualised on a $2 \%$ agarose gel... 119 Figure 5.11. PCR to confirm gene deletion of hpuA gene in NZ97/052 isolate, visualised on a $2 \%$ agarose gel. .121

Figure 5.12. Deletion of fetA and fur from NZ97/052 does not impact cell migration inhibition.

Figure 5.13. Deletion of $h p u A$ and the double mutant $h p u A B$ from NZ97/052 significantly reduces cell migration inhibition, but deletion of $h p u B$ alone does not.

Figure 5.14. Addition of $20 \mu \mathrm{M} \mathrm{FeSO}_{4}$ to the cell culture medium resulted in cell migration inhibition by NZCM112 and NZ97/052 $\triangle$ hpuA. .126

Figure 6.1. Haptoglobin supplementation increases the ability of NZ97/052 $\triangle h p u A$ to inhibit wound repair. 139

Figure 6.2. Human and bovine haptoglobin levels remain constant in bacteria and host cell coculture. .141 
Figure 6.3. Fluorescence microscopy showing haptoglobin localisation in $16 \mathrm{HBE}$ and bacteria coculture. .143

Figure 6.4. Purified human haptoglobin does not exhibit any antimicrobial effects on meningococcal isolates. 145

Figure 6.5. NZCM112 has a reduced growth rate compared with NZ97/052 and NZ97/052 $\Delta h p u A$ in standing liquid culture over a time course.

Figure 6.6. Growth of NZCM112 is lower than NZ97/052 and NZ97/052 $\Delta h p u A$ in the presence of $16 \mathrm{HBE}$ cells. 150

Figure 6.7. Both NZCM112 and NZ97/052 $\triangle$ hpuA are associated with $16 \mathrm{HBE}$ cells at lower rates.

Figure 6.8. NZ97/052 invades epithelial cells at a higher rate than NZCM112 or NZ97/052

\hpuA. .154

Figure 6.9. Fluorescence microscopy showing association of NZCM112, NZ97/052, and NZ97/052 $\triangle h p u A$ isolates with 16HBE cells. .156

Figure 6.10. SDS-PAGE gel of total cell protein fraction for $E$. coli induced with 0.4 mM IPTG or repressed with $1 \%$ glucose for one hour or three hours. 159

Figure 6.11. Growth curve of BL21 E. coli associated to 16HBE cells. 161

Figure 6.12. Expression of HpuA in BL21 E. coli results in increased levels of cell association with $16 \mathrm{HBE}$ cells. 163

Figure 6.13. Protein sequence alignment of HpuA from N. meningitidis isolates. .165

Figure 6.14. Protein sequence alignment of HpuA homologues. 166

Figure 9.1. Assay testing leakage between apical layer and basal layer in Transwell system.... 211

Figure 9.2. Sequence of $h p u A$ region from NZ97/052 $\triangle h p u A$ gene deletion mutant. 216

Figure 9.3. Sequence of $h p u B$ region from NZ97/052 $\triangle h p u B$ gene deletion mutant. 217

Figure 9.4. Sequence of $h p u A B$ region from NZ97/052 $\triangle h p u A B$ gene deletion mutant. 218

Figure 9.5. Gene expression of CysG and NMBNZ0533_0037 in NZ97/192 household isolates.

Figure 9.6. Nucleotide and predicted protein sequences of $h p u A$ in NZ97/052. 224

Figure 9.7. Codon optimised nucleotide sequence and predicted protein sequence of $h p u A . .225$ 
Figure 9.8. Protein sequence alignment of HpuA sequence in NZ97/052 and codon optimised

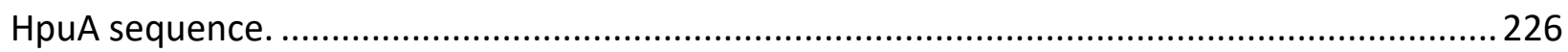




\section{List of Tables}

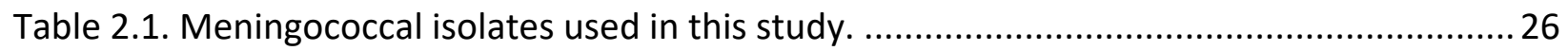

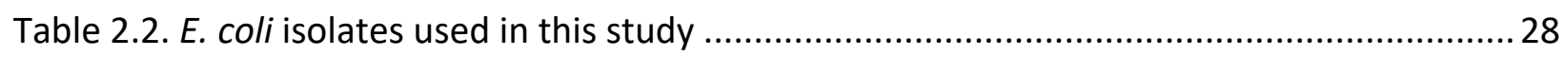



Table 2.4. Oligonucleotide primers used for mutagenesis.....................................................34

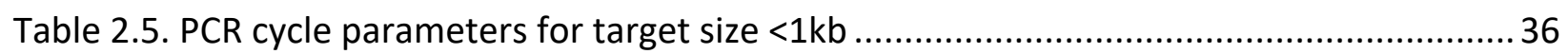

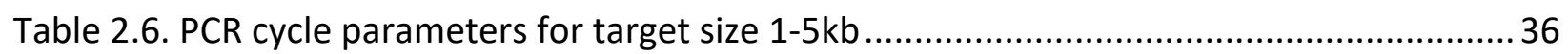

Table 5.1. Number and types of nucleotide variants which occur between isolates in the

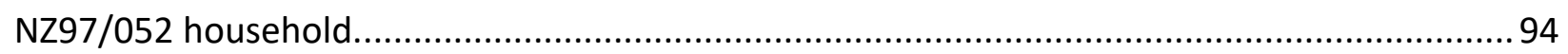

Table 5.2. Variants among the NZ97/052 household. .................................................... 95

Table 5.3. Chromosomal location of the gene, predicted cellular location of the encoded protein, and functional classification of the 48 of the most highly differentially expressed transcripts between isolates NZ97/052 and NZCM112. ............................................... 105 Table 9.1 RNA-seq expression levels of 48 genes most differentially expressed between NZ97/052 and NZCM112.

Table 9.2 Genes previously deleted from disease-associated meningococcal isolates, which did

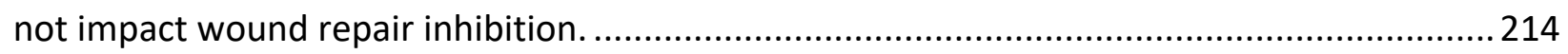



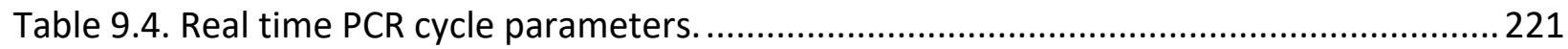




\section{List of Abbreviations}

16HBE

$\mathrm{Amp}^{\mathrm{R}}$

ANOVA

BBB

bp

BLAST

BHI

CC

CBA

CFU

CSF

DAPI

$\mathrm{dH}_{2} \mathrm{O}$

DNA

DMSO

DUS

E. coli

ECM

EDTA

ELISA

ESR

FBS

$\mathrm{Fe}^{2+}$

$\mathrm{Fe}^{3+}$

Fw

gDNA

IPTG
Human bronchial epithelial cell line 16HBE14o-

Ampicillin resistance

Analysis of variance

Blood-brain barrier

Base pair

Basic Local Alignment Search Tool

Brain heart infusion

Clonal complex

Columbia blood agar

Colony forming unit

Cerebrospinal fluid

4',6-diamidino-2-phenylindole

Distilled water

Deoxyribonucleic acid

Dimethyl sulfoxide

DNA uptake sequence

Escherichia coli

Extracellular matrix

Ethylenediaminetetraacetic acid

Enzyme-linked immunosorbent assay

Institute of Environmental Science and Research

Foetal bovine serum

Iron (II) ion

Iron (III) ion

Forward primer

Genomic DNA

Isopropyl $\beta$-D-1-thiogalactopyranoside 


\begin{tabular}{|c|c|}
\hline $\operatorname{Kan}^{\mathrm{R}}$ & Kanamycin resistance \\
\hline $\mathbf{K b}$ & Kilobase \\
\hline kDa & Kilodalton \\
\hline Km & Kanamycin \\
\hline LB & Luria-Bertani broth \\
\hline LPS & Lipooligosaccharide \\
\hline MenB & Meningitis serogroup B \\
\hline MLST & Multilocus sequence typing \\
\hline MOI & Multiplicity of infection \\
\hline mRNA & Messenger RNA \\
\hline MRL & Meningococcal Reference Laboratory \\
\hline Mw & Molecular weight \\
\hline N. gonorrhoeae & Neisseria gonorrhoeae \\
\hline N. lactamica & Neisseria lactamica \\
\hline N. meningitidis & Neisseria meningitidis \\
\hline NB & No bacteria \\
\hline NEAA & Non-essential amino acids \\
\hline NZ & New Zealand \\
\hline NZGL & New Zealand Genomics Limited \\
\hline$O_{600}$ & Optical density at $600 \mathrm{~nm}$ \\
\hline ORF & Open reading frame \\
\hline PBS & Phosphate-buffered saline \\
\hline PCR & Polymerase chain reaction \\
\hline qRT-PCR & Quantitative real-time PCR \\
\hline RNA & Ribonucleic acid \\
\hline RNA-seq & RNA sequencing \\
\hline Rv & Reverse primer \\
\hline S. pyogenes & Streptococcus pyogenes \\
\hline SD & Standard deviation \\
\hline
\end{tabular}




$\begin{array}{ll}\text { SDS } & \text { Sodium dodecyl sulfate } \\ \text { SDS-PAGE } & \text { Sodium dodecyl sulfate polyacrylamide gel electrophoresis } \\ \text { SNP } & \text { Single nucleotide polymorphism } \\ \text { ST } & \text { Sequence type } \\ \text { TCP } & \text { Total cell protein } \\ \text { TE } & \text { Tris-EDTA } \\ \text { VUW } & \text { Victoria University of Wellington } \\ \text { WT } & \text { Wild-type }\end{array}$




\section{Introduction}

\subsection{Neisseria meningitidis}

Neisseria meningitidis, known as meningococcus, is a gram-negative aerobic diplococcoid bacterial species, which resides in the mucous membranes of the nasopharynx. N. meningitidis is the causative agent of meningococcal disease, of which the first description was published by Vieusseux (1805). Gram-negative diplococci were first observed in cerebrospinal fluid from a meningitis case by Marchiafava and Celli (1884). Anton Weichselbaum discovered Neisseria meningitidis as the causative agent of meningococcal disease in 1887 , by isolating the bacterium from cerebrospinal fluid collected from six meningitis patients (Weichselbaum, 1887). The bacterium was first named Diplococcus intracellular meningitidis, but subsequently changed to Neisseria meningitidis.

N. meningitidis can be considered an obligate human commensal pathogen. Commensal pathogens are bacteria which are commonly found as asymptomatic colonisers, have humans as their normal ecological niche, and cause disease rarely, relative to healthy carriage (Henriques-Normark \& Normark, 2010). However, various microbial, environmental or host factors can trigger bacterial infection or invasive disease. In the case of $N$. meningitidis, the bacteria can occasionally cause meningococcal meningitis and septicaemia (Mietzner \& Morse, 2010; Stephens, 2009).

\subsubsection{Bacterial colonisation}

Humans are the only natural host of meningococci, with no other known reservoirs (Rosenstein et al., 2001). N. meningitidis is transmitted through inhalation of respiratory droplets or direct contact with saliva or nasal secretions, however, the inoculum size required for transmission is currently unknown (Tzeng \& Stephens, 2000). Acquisition of meningococci may lead to transient colonisation, invasive disease, or longer term colonisation or carriage (Stephens et al., 2007). 
The colonisation of upper respiratory mucosal surfaces by N. meningitidis is the first step in the establishment of carriage and is a prerequisite for invasive meningococcal disease (IMD) (Townsend et al., 2002). Colonisation is an immunising process that can help prevent future disease, due to the induction of local antibody production at the mucosal surface (Horton et al., 2005). Colonising meningococci must attach to the mucosal surface, utilise local nutrients and evade host immune defences (Yazdankhah \& Caugant, 2004). The host immune status, integrity of the mucosa, and bacterial factors are key to determining if meningococci will cause invasive disease or colonise asymptomatically (Mueller \& Gessner, 2010).

Colonisation by $N$. meningitidis begins with the selective adherence of viable meningococci to non-ciliated mucosal epithelial cells through multiple adhesion factors (Stephens \& Farley, 1991; Stephens et al., 1983). Adhesion to epithelial surfaces is vital for the survival of meningococci; virulence factors that mediate attachment and colonisation of upper respiratory airways are essential for disease (Nagele et al., 2011). Several meningococcal adhesins recognising host receptors have been identified (Hung \& Christodoulides, 2013; Merz \& So, 2000). Type IV mediated pili are considered the main meningococcal adhesions, which target the CD46 receptor, a human cell surface protein (Carbonnelle et al., 2006; Johansson et al., 2003; Kallstrom et al., 1998). The opacity proteins, Opa and Opc, are also involved in bacterial attachment to host cells and target CD66 and heparin receptors respectively (de Vries et al., 1998; Virji et al., 1999; Virji et al., 1993). Minor adhesins include Neisserial adhesin A (NadA) and Neisseria hia/hsf homologue (NhhA) (Comanducci et al., 2002; Scarselli et al., 2006). At the site of initial attachment, meningococci proliferate on the surface of non-ciliated epithelial cells and form microcolonies (Coureuil et al., 2014).

\subsubsection{N. meningitidis carriage}

In 1896, F. Keifer cultured N. meningitidis collected from the nasopharynx of disease patients and their close contacts. He discovered that healthy individuals could be asymptomatic nasopharyngeal carriers (1896). Nasopharyngeal carriage of meningococci is relatively common; 
with approximately $10-15 \%$ of healthy individuals carrying N. meningitidis asymptomatically in non-epidemic periods, and up to $30 \%$ of healthy individuals in epidemic periods (Capecchi et al., 2005; Claus et al., 2002; Nagele et al., 2011; Stephens \& Farley, 1991; Tzeng \& Stephens, 2000). Meningococcal carriage in infants is almost absent, peaking to $\sim 25 \%$ in young adults, and eventually decreasing to 8\% in older adults (Christensen et al., 2010). However, carriage rates, determined by traditional swab, may underestimate actual carriage; a previous study has suggested up to $75 \%$ of carriers may be missed by this detection technique (Sim et al., 2000).

N. meningitidis strains, carried asymptomatically, are eventually cleared and the duration of carriage can vary from several days to several months. Carriage lasts for months in about $25 \%$ of cases, is intermittent in 35\%, and is short-lived or infrequent in the remaining $40 \%$ (Broome, 1986; Stephens, 1999). Individuals may be carriers of various meningococcal strains over their lifetime in multiple asymptomatic episodes (Ala'Aldeen et al., 2000; Altmann et al., 1973). In very rare instances, simultaneous carriage of multiple meningococcal strains has been described (Andersen et al., 1998; Hill et al., 2010). Higher levels of meningococcal carriage typically coincide with higher meningococcal disease rates, however, incidence of invasive disease cannot be accurately predicted from carriage levels (Caugant et al., 2007).

The high rate of asymptomatic carriage has triggered investigations into why $N$. meningitidis causes disease and mortality in some, while remaining completely innocuous in others. When compared to the prevalence of transmission and carriage of $N$. meningitidis, invasive disease is uncommon. This can be partially explained by the low pathogenicity of some meningococcal isolates. Most strains of $N$. meningitidis isolated from the nasopharynx of carriers have limited potential for pathogenicity; thus invasive disease is relatively uncommon. There is considerable variability in the genomes of meningococcal isolates which have been recovered from asymptomatic carriers (Caugant, Froholm, et al., 1986). In most cases, invasive disease is caused by a few "hypervirulent" lineages (Claus et al., 2003). There is substantial genetic diversity between isolates which cause invasive disease and those which are carried asymptomatically (Stephens, 2009). 
Virulence by $N$. meningitidis is polygenic (Joseph et al., 2010), which means there are likely many virulence factors that contribute to the hypervirulent phenotype. This is in contrast with other bacteria which have discreet pathogenicity islands, toxins, or other virulence factors that clearly differentiate commensal strains from virulent strains (Gal-Mor \& Finlay, 2006; Wilson et al., 2002). Factors which help N. meningitidis colonise the nasopharynx are adaptive traits, which enable the bacteria to survive and replicate. Causing invasive disease may not be advantageous to meningococci as this often leads to death of the host, which is frequently a dead-end for the bacteria (Klughammer et al., 2017; Meyers et al., 2003). N. meningitidis virulence may be an accidental side effect of other advantageous phenotypes (Brown et al., 2012). For instance, meningococci may avoid detection or clearance by the host immune system by invading epithelial cells, which can lead to eventual invasion of the bloodstream Therefore, many 'virulence factors' that contribute to invasive disease are often present in both virulent and benign isolates (Snyder \& Saunders, 2006). Due to this, comparing many disease and carriage meningococcal genomes has not effectively yielded virulence factors strictly related to invasive disease.

In 2001, a study in Auckland, New Zealand was published, comparing strains of $N$. meningitidis isolated from patients with meningococcal disease, to those isolated from healthy household contacts (Simmons et al., 2001). The results of this study found in 28 of the 52 households surveyed, at least one carriage isolate was indistinguishable, by standard laboratory serological and sequence typing, from the associated disease isolate (Simmons et al., 2001). These isolates are presumed closely related, belonging to the same clonal complex. However these isolates are not identical as phenotypic differences, such as TNF- $\alpha$ secretion and intracellular glutathione levels, have been observed (Ren et al., 2017). Studying closely related meningococcal isolates, such as these household contact $N$. meningitidis disease-carriage pairs, has proved to be a very useful technique to identify disease-associated virulence factors that cannot be found by comparing large numbers of meningococcal genomes (Ren et al., 2017). 


\subsubsection{Biology of $N$. meningitidis and bacterial surface structures}

N. meningitidis isolates may be encapsulated or non-capsulated. The meningococcal capsule, arguably the most important $N$. meningitidis virulence factor, helps not only with transmission and colonisation, but also protects against desiccation, phagocytic killing, and killing by complement and opsonisation (Moxon \& Kroll, 1990; Stephens, 2009; Stephens et al., 2007). Invasive strains are all encapsulated, whereas all non-capsulated $N$. meningitidis are associated with asymptomatic carriage, and rarely cause disease (Claus et al., 2002; Johswich et al., 2012; Vogel \& Frosch, 1999). By protecting the bacteria, from both phagocytes and complement, the capsule is essential for rapid replication in the bloodstream, the hallmark of IMD. Isolates collected from asymptomatic carriers may be non-groupable (without a capsule), or express various capsules (Claus et al., 2002; Tzeng \& Stephens, 2000; Yazdankhah \& Caugant, 2004). The capsule is therefore necessary for IMD, but not sufficient for virulence.

Environmental adaptation by bacteria contributes to invasive potential. $N$. meningitidis frequently modifies the expression of outer membrane structures to avoid detection by the host immune system. Modulation can occur through phase variation, antigenic variation, or recombination (Caugant, Bovre, et al., 1986; Hill et al., 2010). Phase variation is a reversible switch between an on and off expression phase of a gene (van der Woude \& Bäumler, 2004). Phase variable genes are often controlled by short sequence repeats, positioned upstream, or within the gene of interest. These repeats are prone to mispairing during DNA replication, which can lead to translational changes (Henderson et al., 1999). Antigenic variation refers to the modification in expression of immunogenic modules on the surface of the bacteria, such as the alternate switching between the main pilin antigen, that occurs frequently in vivo in both $N$. meningitidis and N. gonorrhoeae (Vink et al., 2012). N. meningitidis can use antigenic variation to modulate virulence by evading host immune systems (Nassif et al., 1993). Recombination is the exchange of genetics between different organisms which leads to variation (Eisenstark, 1977). 


\subsubsection{Iron availability in the human host and bacterial iron acquisition}

Transition metals are essential for bacterial survival, as they are crucial in many biological processes (Andreini et al., 2008). The human body is a rich source of nutrients, and limiting bacterial access to nutrients is an important host defence (Wallace, 2016; Weinberg, 1975, 2009). 'Nutritional immunity' is the process by which the host prevents growth of invading pathogens by restricting access to essential nutrient metals (Hood \& Skaar, 2012; Stork et al., 2013). Iron in particular is known to contribute to meningococcal pathogenesis (Perkins-Balding et al., 2004). Animal models have demonstrated that pre-treatment of mice with iron increased the lethality of invasive disease following infection with N. meningitidis (Holbein, 1981).

Humans and other mammals utilise iron-binding proteins and iron-sequestering compounds to regulate free iron and keep levels low. There are many mechanisms by which the host withholds iron from bacteria, including storage of Iron(III) $\left(\mathrm{Fe}^{3+}\right)$ intracellularly, complexed with ferritin (Torti \& Torti, 2002). $\mathrm{Fe}^{3+}$ can also be bound by serum transferrin or lactoferrin at mucosal surfaces (Crichton \& Charloteaux-Wauters, 1987; Lonnerdal \& Iyer, 1995). Iron(II) $\left(\mathrm{Fe}^{2+}\right)$ is complexed with haem in the blood, which is then bound by haemoglobin within red blood cells (Hood \& Skaar, 2012). Approximately 65\% of the iron in the human body is stored within haemoglobin (Andrews, 1999). Haptoglobin binds free haemoglobin to form haptoglobin-haemoglobin complexes, which can prevent scavenger microbes from utilising haemoglobin as an iron source (Wassell, 2000).

Although iron is the most copious transition metal in the human host, free ferrous iron $\left(\mathrm{Fe}^{2+}\right)$ is not readily available (Porcheron et al., 2013). The level of free iron in mammals varies throughout the body but the mean value is $10^{-18} \mathrm{M}$, much lower than the $10^{-6} \mathrm{M}$ level required for optimal bacteria growth (Rohmer et al., 2011). The acquisition of nutrients by bacteria is critical in the pathogenesis of infectious disease (Hood \& Skaar, 2012). Successful bacterial colonisers have developed strategies to overcome the limitations of nutrient availability in order to exploit host resources (Perkins-Balding et al., 2004). Bacterial iron acquisition can be 
divided into free $\mathrm{Fe}^{2+}$, haem, and siderophore acquisition systems (Rohmer et al., 2011). Lactoferrin predominates as the main source of iron in the nasopharynx, which is the entry site of N. meningitidis (Perkins-Balding et al., 2004). Intracellular meningococci have also been shown to utilise ferritin as a critical source of iron (Larson et al., 2004). N. meningitidis is able to bind transferrin, lactoferrin, haemoglobin, and haptoglobin-haemoglobin (Weinberg, 2009). The transferrin receptor consists of $\mathrm{Tbp} A$ and $\mathrm{TbpB}$, while the lactoferrin binding receptor is made up of proteins LbpA and LbpB (Noinaj et al., 2012; Perkins-Balding et al., 2004). HmbR functions as a Neisseria haemoglobin receptor (Stojiljkovic et al., 1996), and the HpuAB receptor, consisting of proteins $\mathrm{HpuA}$ and $\mathrm{HpuB}$, is able to bind both haemoglobin and haptoglobinhaemoglobin complexes (Lewis et al., 1999). Many bacteria use siderophore mediated uptake. Siderophores are low molecular weight iron chelators secreted by bacteria, which can bind $\mathrm{Fe}^{3+}$ with a higher affinity than transferrin and lactoferrin (Schalk, 2008). Siderophores acquire iron from host proteins or low molecular mass iron compounds. Although N. meningitidis does not appear to produce siderophores, research suggests meningococci may be able to utilise siderophores secreted by other bacteria (Archibald \& DeVoe, 1979; Rutz et al., 1991).

\subsubsection{Invasive meningococcal disease}

N. meningitidis is a much-feared bacterial pathogen, due to the rapid onset of disease and high mortality rate. IMD can have extremely rapid onset; an individual can progress from healthy to severely ill in a matter of hours. Death can occur 24 to 48 hours after the onset of symptoms (Thompson et al., 2006). Emergence of the disease in previously healthy young adults and children has led to increasing public awareness and research.

Meningococcal disease is used to describe two different serious illnesses which can be caused by $N$. meningitidis; meningitis and septicaemia. Meningococcal meningitis results from meningococci multiplying in the cerebrospinal fluid (CSF) and meninges, which can be fatal due to raised intracranial pressure. Meningococcal septicaemia describes a pathogenic level of bacteria in the bloodstream and can lead to death due to septic shock and cardiovascular failure. Sepsis is recognised to be more dangerous than meningitis, and much of the disease 
pathology is due to the vigorous host immune response (Baraff et al., 1993; Hart \& Thomson, 2006; Mietzner \& Morse, 2010). Patients can present with either illness, with approximately $40 \%$ of IMD patients presenting with both infections (Mehta \& Levin, 2000). Other less common outcomes of meningococcal disease include septic arthritis, pneumonia, purulent pericarditis, conjunctivitis, otitis, sinusitis, and urethritis (Tzeng \& Stephens, 2000; Yazdankhah \& Caugant, 2004). A rare condition, chronic meningococcemia, may present as fever, rash, joint aches and headaches for several weeks (Rosenstein et al., 2001; Tzeng \& Stephens, 2000).

Meningococcal disease occurs one to 14 days after pathogen acquisition (Rosenstein et al., 2001; Stephens, 1999). The disease mortality rate of IMD is high, at approximately $10 \%$, although the range has been reported from 5-20\% (Franzoso et al., 2008; Tzeng \& Stephens, 2000). In New Zealand, the case mortality rate has varied from 4-10\% in the past decade (Lopez \& Sherwood, 2014). This rate remains relatively high, even with prompt antibiotic intervention, due to the rapid progression from IMD to severe meningitis or septicaemia (Tzeng \& Stephens, 2000). Infections can cause multi-organ failure and death within hours of initial presentation, therefore, antibiotic therapy is often administered too late. The high rate of mortality can also be partly attributed to the difficulty of early diagnosis. Early stage symptoms are often nonspecific, such as fever, muscle pain, headache, and respiratory or gastro-intestinal symptoms (Nield \& Weisse, 2008; Pace \& Pollard, 2012). These symptoms resemble many other milder illnesses, such as influenza. By the time symptoms specific to meningococcal disease emerge, such as a rash, neck stiffness, and light sensitivity, treatment is much less effective (Rosenstein et al., 2001; van de Beek et al., 2004). Of those that do survive IMD, up to $25 \%$ suffer from serious life-long sequelae, such as hearing loss, behavioural problems, cognitive dysfunction, seizure disorder, and limb amputation (Comanducci et al., 2004; Mietzner \& Morse, 2010; Stephens, 2009; Tzeng \& Stephens, 2000). The societal impact of meningococcal disease is therefore higher than the annual incidence. The high possibility of adverse outcomes is one reason that preventative measures, such as vaccines, are favoured. 


\subsubsection{Progression of invasive meningococcal disease}

N. meningitidis can be classified as a commensal organism which may become a dangerous pathogen (Comanducci et al., 2004). Four conditions must be met for invasive disease to occur: exposure to bacteria, colonisation resulting in transient or long-term carriage, passage through the mucosa, and survival in the bloodstream (van Deuren et al., 2000). Under certain conditions, N. meningitidis can navigate across the mucosal barrier of the nasopharynx and gain access to the bloodstream, causing bacteraemia (Rosenstein et al., 2001). If the bacteria survive in the bloodstream and cross the blood-brain barrier (BBB), to reach the meninges, meningitis is likely to occur (Mietzner \& Morse, 2010). Invasive disease caused by N. meningitidis, as with other bacterial pathogens, is a complex, multistep process (Finlay \& Falkow, 1997).

Meningococcal disease occurs when colonising bacteria cross the mucosal barrier and enter the bloodstream (Figure 1.1). Access to the bloodstream can be gained by directly invading damaged epithelial surfaces, crossing the epithelium through phagocytes, or via the M cells of the nasal-epithelium-associated lymphoid tissue in the adenoids and tonsils (Stephens et al., 2007; Virji, 2009). N. meningitidis can be found inside non-ciliated epithelial cells, therefore, it has been theorised this may be a mechanism to invade tissues (Stephens et al., 1983). The bacteria may use parasite-directed phagocytosis to enter these respiratory cells and transcytose to underlying submucosal tissues, or they may penetrate between epithelial intracellular junctions. Alternatively, they may be carried across epithelial barriers within cells (Pizarro-Cerda \& Cossart, 2006; Stephens, 2009). N. meningitidis is a human-adapted pathogen (Stephens, 2009). Like other human-adapted pathogens, most of the meningococcal virulence factors target human proteins and disable or compromise elements of the human immune response (Hill et al., 2010). For this reason, there are no animal models that are able to reproduce the natural course of disease and transmission, and as consequence, the mechanism used to invade mucosal tissues is not well understood. Many benign carriage-associated isolates are also highly invasive of epithelial cells in vitro, but rarely cause disease. Therefore, intracellular invasion might be employed as one means to avoid the host immune response, rather than as a route into the bloodstream. 


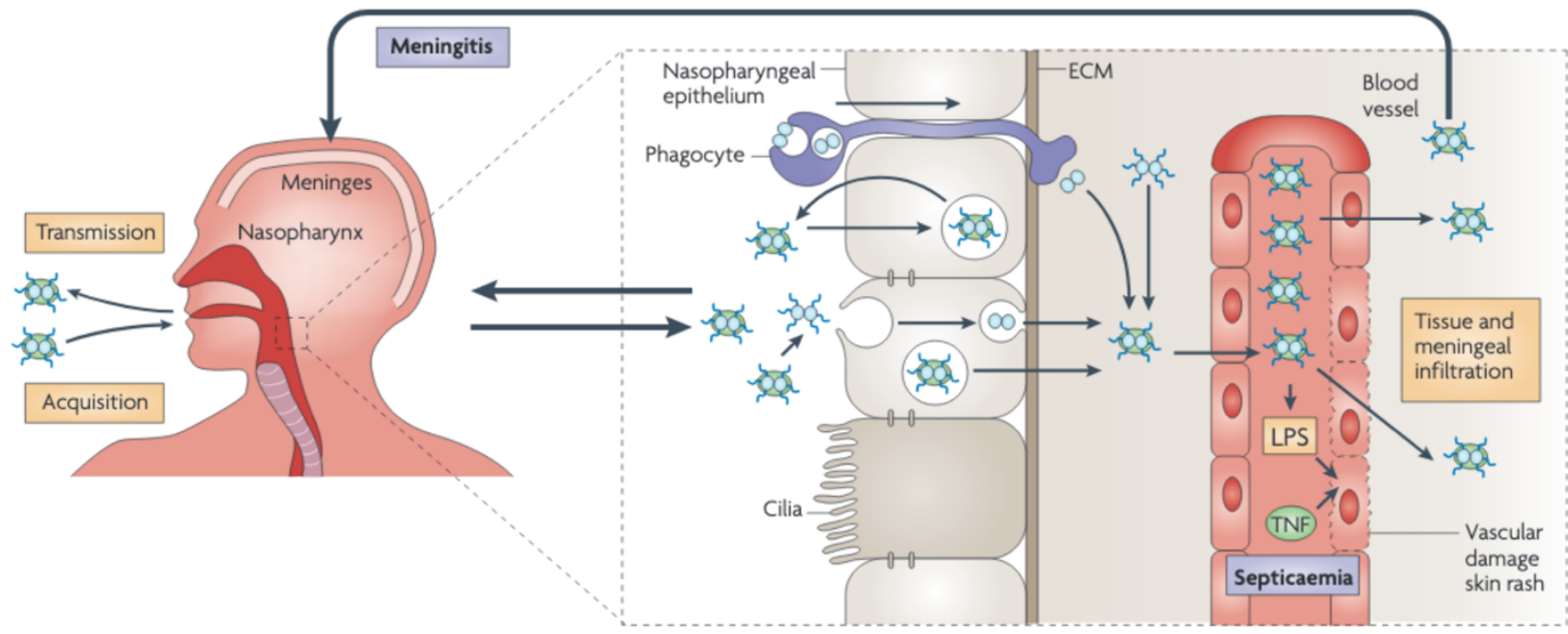

Figure 1.1 Stages of pathogenesis for $\boldsymbol{N}$. meningitidis. N. meningitidis attaches to non-ciliated mucosal epithelial cells. Bacteria may bypass the epithelial layer by transcytosis, through phagocytes, or crossing directly following loss of integrity of the mucosal epithelium. Reproduced with permission from 'Pathogenic neisseriae: surface modulation, pathogenesis and infection control' Virji M, (Nature Reviews Microbiology 2009; 7,4: 274-286) (Virji, 2009), Copyright Springer Nature.

In order to survive within the bloodstream, N. meningitidis must replicate rapidly, while avoiding host immune responses and phagocytic killing. Bacterial multiplication in the bloodstream is associated with the release of host cytokines, including IL-1, IL-6, and TNF- $\alpha$. Release of these cytokines is known to be important in the development of meningococcal sepsis (Brandtzaeg et al., 1992). Meningococcal infections are most often not discovered until infection of the CSF and presentation of meningitis, suggesting that bacteria in the bloodstream may be asymptomatic until they cross the BBB. However, in other patients, $N$. meningitidis in the bloodstream leads to sepsis. These observations highlight how little is known about how the bacteria causes invasive disease.

Most invasive bacteria that reach the bloodstream are prevented from crossing the BBB or infecting the central nervous system (Coureuil et al., 2013). N. meningitidis is one of the few pathogens which is able to cross the BBB and replicate in the CSF. Inside the CSF, bacterial multiplication is largely uncontrolled due to the lack of local complement and immunoglobulins. 
It is not fully understood how meningococci cross the BBB, but it is thought inflammatory cytokines may cause leakage of the barrier (Brandtzaeg et al., 1992). Much remains unknown about how $N$. meningitidis causes IMD, and there are still many mysteries about which bacterial factors can trigger invasive disease. Although most meningococcal strains isolated from healthy individuals rarely cause disease, it is clear certain surface structures that facilitate attachment to host cells, such as pili and adhesins, are important virulence factors.

\subsubsection{Epidemiology of $N$. meningitidis}

N. meningitidis isolates can be classified into 13 identified serogroups based on the immunological reactivity and biological composition of the polysaccharide capsule (Rosenstein et al., 2001; Stephens et al., 2007). Serogroups A, B, C, W-135, X, and Y are responsible for most instances of invasive and potentially fatal meningococcal disease worldwide, accounting for approximately $90 \%$ of cases (Stephens et al., 2007). Serogroup B is responsible for more than $50 \%$ of IMD in the world, and the majority of cases of meningococcal septicaemia and meningitis in developed countries (Comanducci et al., 2002; Franzoso et al., 2008; Tavano et al., 2011).

Further distinguishing features of $N$. meningitidis include outer membrane structures (Figure 1.2). Outer membrane proteins PorB and PorA, determine serotype and serosubtype respectively (Feavers et al., 1996; Jolley et al., 2007). This has led to a naming system of meningococci classified by their serogroup, serotype, and serosubtype (Figure 1.3). 


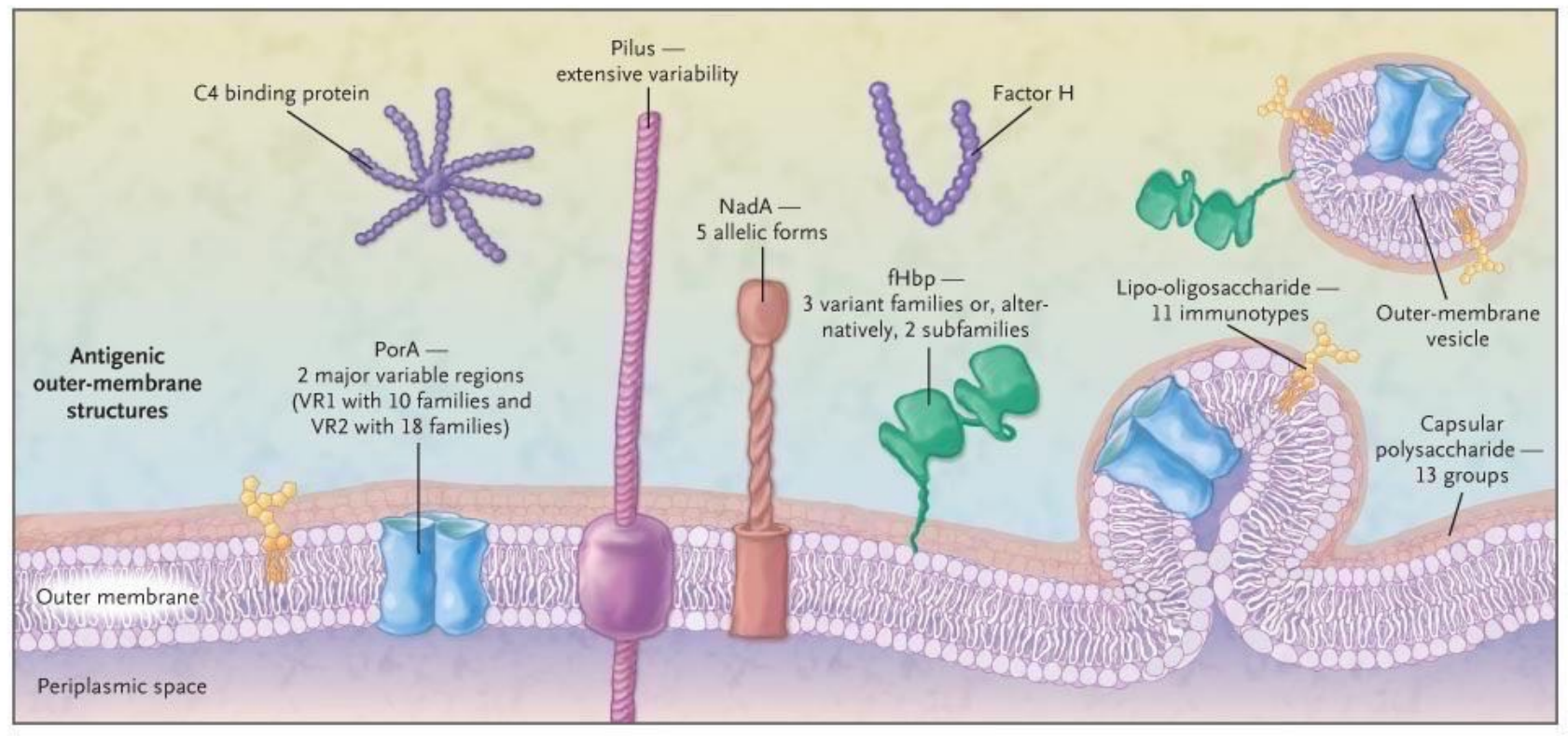

Figure 1.2 Common features of the meningococcal outer membrane. NadA is a meningococcal adhesin. fHbp binds factor $\mathrm{H}$, an inhibitory protein of the complement system. Reproduced with permission from 'Advances in the Development of Vaccines against Neisseria meningitidis' Tan L.K.K. et al, N Engl J Med 2010; 362:1511-1520)(Tan et al., 2010), Copyright Massachusetts Medical Society. 


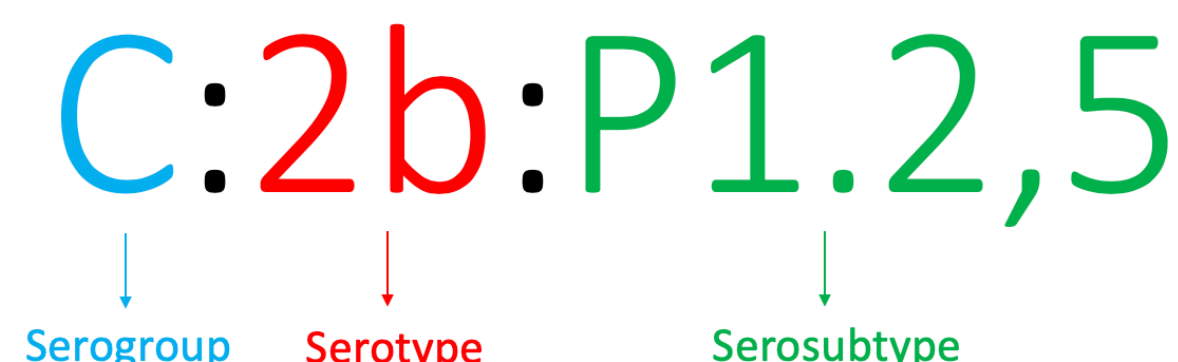

Figure 1.3 Example of Serogroup-Serotype-Serosubtype classification of $\boldsymbol{N}$. meningitidis. Serogroup corresponds to the polysaccharide capsule. Serotype corresponds to the porB allele, and serosubtype to the porA allele.

N. meningitidis is naturally competent and highly naturally transformable, so horizontal gene transfer is a major driver of genome heterogeneity (Schoen et al., 2009). N. meningitidis has been documented to be able to take up DNA from the environment, other meningococcal isolates, carriage Neisseria species, and even other non-Neisseria species (Beddek et al., 2009). Most phylogenetic trees of Neisseria isolates are rootless, which indicates how frequently DNA is taken up and integrated into the genome by homologous recombination. Due to frequent variation of surface structures, a commonly used typing method of $N$. meningitidis is multi locus sequence typing (MLST), which is used to infer genome phylogeny (Maiden et al., 1998; Spratt, 1999). MLST was pioneered in N. meningitidis but is now widespread for other bacterial species. This approach uses the DNA sequence of seven housekeeping genes: $\operatorname{aroE,}$ gah, fumC, $p d h C, a b c Z, a d k$, and pgm. A 450-500 bp internal fragment from each gene is sequenced and assigned an allele number (Maiden et al., 1998). The combination of allele numbers from each locus determines the sequence type. Sequence types that are closely related are grouped into lineages, or clonal complexes (CC) (Feil et al., 1999). Most laboratory typing is now performed by sequencing, but the original serological terminology still persists. 


\subsubsection{N. meningitidis transmission}

Transmission is essential for the life cycle of N. meningitidis (Yazdankhah \& Caugant, 2004). In open populations, the $N$. meningitidis transmission rate is relatively low. Transmission rates are significantly increased in populations living in confined areas, such as military recruits and university students (Harrison et al., 1999; Stephens, 1999). Enclosed populations lead to an increased chance of transmission of bacteria, and carriage rates can reach as high as $70 \%$ (Bidmos et al., 2011; Caugant et al., 1992). Meningococcal disease can present as sporadic cases or as outbreak clusters in both developing and developed countries. The normal incidence of meningococcal disease is between $0.5-5$ cases per 100,000 individuals in the developed world, although these rates vary geographically (Comanducci et al., 2002; Sun et al., 2013; Yazdankhah \& Caugant, 2004). Approximately $1,200,000$ cases of IMD occur annually, resulting in 135,000 deaths per year worldwide (Harrison et al., 2009; Rouphael \& Stephens, 2012).

Epidemic outbreaks are a key characteristic of meningococcal disease (Stephens, 2009); during an epidemic, incidence rates can rise above 15 , up to 400 , cases per 100,000 people (Comanducci et al., 2002). The region known as the "meningitis belt", which is defined by latitude and extends through sub-Saharan areas of Africa, has historically had the highest disease rates in the world (Figure 1.4). Rates of over 1,000 cases per 100,000 individuals have been recorded, a number that is elevated relative to non-epidemic periods in sub-Saharan Africa (Mueller \& Gessner, 2010; Tzeng \& Stephens, 2000; Yazdankhah \& Caugant, 2004). In this region, meningococcal disease can become epidemic at the end of the dry season, typically referred to as "meningitis season" (Mueller \& Gessner, 2010). This has been associated with the Harmattan, a dust-filled wind from the Saharan desert (Agier et al., 2013; Greenwood et al., 1984; Tzeng \& Stephens, 2000). Rates of meningococcal disease have been shown to directly correlate with wind speed and damage to the throat and epithelial layer, without affecting carriage rates (Greenwood et al., 1984; Sultan et al., 2005; Trotter \& Greenwood, 2007). The high mineral dust load, combined with low humidity over several weeks, can cause the mucus membranes to become fragile and can result in tiny wounds and irritations in the pharyngeal mucosa. This provides an opportunity for colonising N. meningitidis to invade deeper tissues 
and reach the bloodstream (Greenwood, 1999; Martiny \& Chiapello, 2013; Mueller \& Gessner, 2010). The majority of IMD cases in the meningitidis belt have historically been caused by serogroup A (Jafri et al., 2013). In recent years, a group A conjugate vaccine, MenAfriVac, has been widely deployed in the African meningitidis belt. This has led to substantial reductions in IMD caused by serogroup A (Trotter et al., 2017).

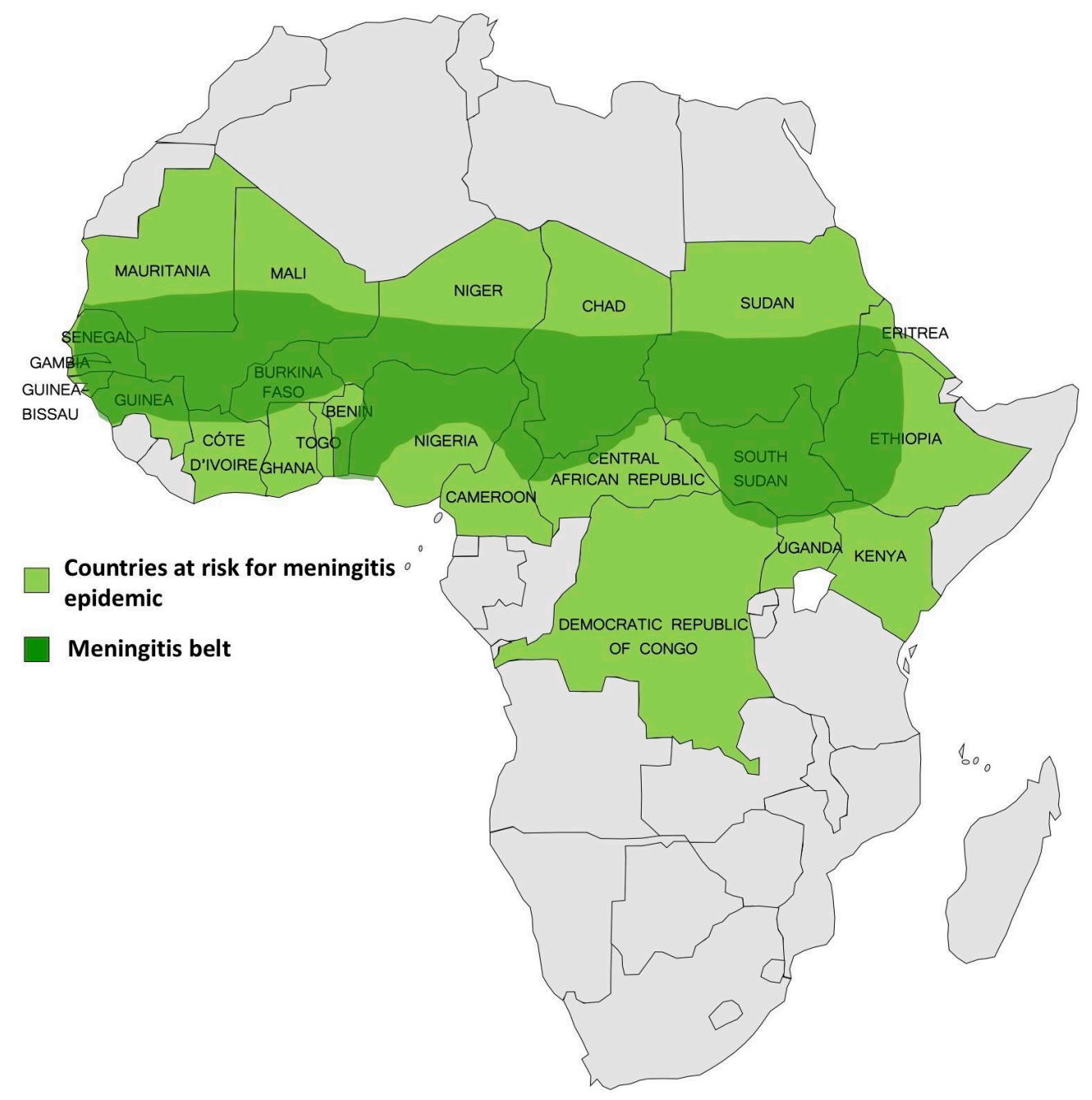

Figure 1.4 Meningitis belt across Africa. Disease data source: World Health Organization. International Travel and Health. Geneva, Switzerland: 2012. (WHO, 2012b). 


\subsubsection{New Zealand and Meningococcal disease}

In New Zealand, the majority of disease cases are caused by serogroups B and C; in 2018 group B was responsible for $45.1 \%$ of disease cases (ESR, 2018b). However, there has recently been an increasing trend of serogroup W-135 meningococcal disease cases in New Zealand (Figure 1.5) (ESR, 2018b). The proportion of serogroup W-135 cases has jumped from $11 \%$ in 2017 to $29.2 \%$ in 2018 (ESR, 2018a).

A New Zealand epidemic of serogroup B occurred between 1991 and 2008, with disease rates reaching 17.4 cases per 100,000 individuals in 2001 (Dyet et al., 2005; Lopez \& Sherwood, 2014; Martin \& Lopez, 2009). During this epidemic, $85 \%$ of disease cases were identified as strain type B:4:P1.7-2,4, and belonged to sequence type (ST) ST-42 or ST-154 of the ST-41/44 clonal complex (Dyet \& Martin, 2006). A specially manufactured vaccine, MeNZB, was produced and administered in a mass vaccination effort from 2004 to 2008. The vaccine was administered to all children in New Zealand between the ages of six weeks and 19 years (Kelly et al., 2007; Martin \& Lopez, 2009). Rates of IMD have since dropped, but localised outbreaks continue to occur, most recently with serogroup W-135 in Northland in 2018 (ESR, 2018b).

In 2018, a total of 115 cases of meningococcal disease, were reported in New Zealand, with 10 deaths (ESR, 2019). This resulted in a national disease rate of 2.5 cases per 100,000 individuals, with the highest number of cases in Northland, where disease rates reached 7.4 cases per 100,000 individuals (ESR, 2019). In response to the Northland outbreak, a vaccination programme was launched to target the most vulnerable population. Free meningococcal W vaccinations were offered to children between nine months and five years, and adolescents between the ages of 13 and 20. 


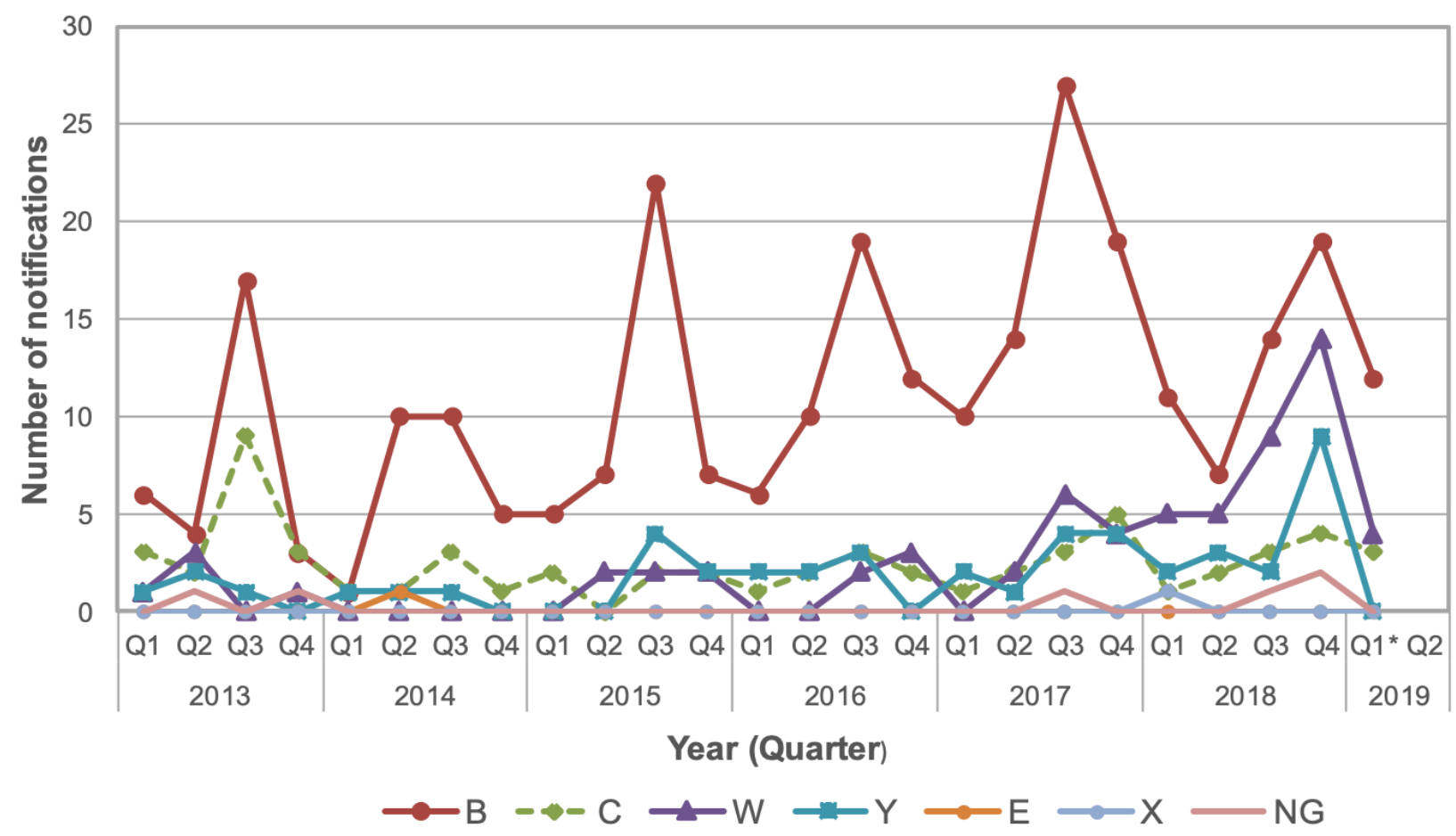

NG - non-groupable.

${ }^{*}$ Cases reported up to 31 March 2019 only.

Figure 1.5 Meningococcal disease notifications in New Zealand by group by quarter by year, 2013-2019 (Cases reported up to 31 March 2019 only). Serogroup B continues to cause the highest number of notifications. However, there is a noticeable rise in IMD notifications caused by serogroup W-135 and Y. Image reproduced from ESR Meningococcal Disease Quarterly Surveillance Report Jan-March 2019, with permission.

\subsubsection{Risk factors for IMD}

There are several factors which play a role in the development of invasive disease, including virulence of the strain, host susceptibility, and environmental influences. Although IMD can affect anyone, age is one of the most important risk factors for disease. In New Zealand, in 2018, the highest rate of meningococcal disease was in infants less than one year old (24.8 cases per 100000 individuals). This is followed by young adults aged 15-19 (5.1 cases per 100 $000)$ and children aged one to four (4.8 cases per 100000$)$ (ESR, 2019). Various other factors can increase the risk of contracting meningococcal disease, including disruption of the nasopharynx epithelium due to smoking or upper respiratory infections, travel to epidemic 
areas, household crowding, and asplenia deficiencies in complement components or mannose binding lectin (Coen et al., 2006; Stephens, 1999, 2009). Environmental factors, such as changes in temperature or humidity, that result in damage to the nasopharynx epithelium, also increase risk of developing IMD (Stephens et al., 2007). IMD risk is also increased with behaviours such as intimate kissing, sharing of drink bottles, and bar patronage (Imrey et al., 1996; Swain \& Martin, 2007; Tully et al., 2006).

Lower socio-economic status is also correlated with higher incidence of IMD (Rosenstein et al., 2001). Meningococcal disease also disproportionately affects certain ethnicities, possibly as a result of genetic predispositions found in specific ethnic groups. For instance, IMD is three-fold more common in New Caledonia than in France. This was found to be due to a high prevalence of complement deficiency status (C-deficiency) in Melanesian patients, particularly those originating from the Loyalty Islands (Daures et al., 2015). In New Zealand, the ethnic groups with the highest rates of disease in 2018 were Pacific peoples (5.1 cases per 100 000) and Māori (4.7 cases per 100 000) (ESR, 2018b). Disease rates for European or Other were 1.9 cases per 100000 individuals (ESR, 2018b). The reasons underlying the differences in prevalence between ethnic groups in New Zealand are not known. They could be impacted by higher rates of smoking, increased respiratory illness, poor quality housing, and household crowding, all of which can inflate disease rates in certain populations.

\subsubsection{Prevention and treatment of $\boldsymbol{N}$. meningitidis}

$N$. meningitidis is primarily controlled by mass vaccination of target groups. Due to the rapid onset of disease and serious sequelae, prevention is a far better option than treatment. Meningococcal disease caused by serogroups A, C, W-135 and $\mathrm{Y}$ are generally preventable by conjugated quadrivalent vaccines targeting their polysaccharide capsules (Deeks, 2010; Lucidarme et al., 2010; Reisinger et al., 2009).

Serogroup B (MenB) capsular polysaccharide is modified with sialic acid, which is frequently found on human cells; as a result, it has low immunogenicity. Furthermore, inducing an immune 
response against the serogroup B capsule would likely induce autoimmunity, which should be avoided (Capecchi et al., 2005; Comanducci et al., 2002; Lucidarme et al., 2010; Montanari et al., 2012). Vaccination against MenB requires stimulation of an immune response to components other than the capsule, such as highly variable outer membrane proteins, for instance, PorA. Subsequently, MenB vaccines have historically been strain-specific, targeting the PorA allele of an outbreak or epidemic strain. Recently, a more universal MenB vaccine has been licensed (Gorringe \& Pajon, 2012). This multi-component recombinant vaccine, Bexsero, is made up of factor $\mathrm{H}$ binding protein ( $\mathrm{fHbp}$ ), neisserial adhesion $\mathrm{A}(\mathrm{NadA})$, Neisseria heparin binding antigen (NHBA), PorA, and outer membrane vesicles (OMV) from New Zealand group B strain NZ98/254 (Gorringe \& Pajon, 2012).

Where meningococcal disease cannot be prevented, prompt diagnosis and antibiotic treatment is extremely important due to rapid disease progression. Antibiotics are used to treat not only individuals with IMD, but also their close contacts. In the case of suspected meningococcal disease, blood, CSF and nasopharyngeal secretions are routinely collected and examined for the presence of microbes. Once diagnosis has occurred, treatment by antibiotics is effective if given in a timely manner (Mietzner \& Morse, 2010). If disease symptoms are suspected during diagnosis, treatment should be given immediately, prior to confirmation of $N$. meningitidis in the CSF (Hase et al., 2014). Previously penicillin was used as the primary antimicrobial agent against $N$. meningitidis (Rosenstein et al., 2001). Recently, however, ceftriaxone has replaced penicillin in New Zealand as the first recommended antibiotic for patients with suspected IMD $(\mathrm{MOH}, 2018)$. Until the $20^{\text {th }}$ century, IMD was associated with mortality rates of up to $70 \%$ (Flexner, 1913; Frosch \& Maiden, 2006). However, antimicrobial therapy, and increased public awareness, have reduced the mortality rate to around 10\% (Franzoso et al., 2008; Tzeng \& Stephens, 2000). 


\subsection{Epithelial cell wound repair and invasive meningococcal disease}

Tight junctions between respiratory epithelial cells act as a physical barrier to prevent invasion by most colonising bacteria (Kojima et al., 2013). These tissues are patrolled by cells of the innate immune system (Parker \& Prince, 2011). The bronchial epithelium, colonised by many types of bacteria, must also have efficient repair machinery to prevent bacterial infiltration of underlying tissue. Immediately following damage to the airway epithelium, epithelial cells function to restore the barrier integrity. In vitro, ex vivo, and in vivo assays have been used to describe the process of wound repair (Coraux et al., 2005; Zahm et al., 1991). The first and most important step of wound repair is epithelial cell migration into the wound area (reepithelialisation), which occurs in the first 24 hours (Perrio et al., 2007; Zahm et al., 1997). Small wounds in the mucosal layer can be efficiently resealed through migration, without the need for cellular proliferation (Dupuit et al., 2000; Leoni et al., 2015). The extracellular matrix, consisting primarily of collagen, fibronectin, elastin, and laminin, functions as a scaffold for migrating cells (Badylak, 2002). RhoA and Rac1 proteins re-organise the cytoskeleton for efficient migration in the appropriate direction (Desai et al., 2004). Proliferation of epithelial cells occurs after 24 hours, but cell differentiation and recovery of pseudostratified epithelium may require weeks (Dupuit et al., 2000; Erjefalt et al., 1995).

Many environmental and host factors that damage the mucosal epithelial surface integrity have been shown to increase the risk for IMD. This includes disruption due to smoking, or exposure to second hand smoke, which may increase the rate of meningococcal disease by $20 \%$ (Coen et al., 2006; Tzeng \& Stephens, 2000). Smoking has been estimated to be responsible for over a third of all IMD cases in the developed world (Fischer et al., 1997). Other causes for respiratory nasopharynx damage, which increases the risk of IMD, include changes in temperature or humidity, and viral or mycoplasma infections (Cartwright et al., 1991; Stephens, 1999, 2009; Stephens et al., 2007). Epidemic periods of IMD correlate with damage to the throat and epithelial layer caused by the Harmattan, in the "meningitis belt" in Africa (Greenwood et al., 1984; Sultan et al., 2005; Trotter \& Greenwood, 2007). Another study demonstrated that $N$. 
meningitidis colonisation actually led to epithelial damage and tissue atrophy, especially in the olfactory epithelial region (Sjolinder \& Jonsson, 2010). Furthermore, it has been found that a polymorphism in the -308 position of the tumour necrosis factor-alpha (TNF- $\alpha$ ) promoter increases the risk of meningococcal disease, without altering the risk of carriage (Read et al., 2009; Westendorp et al., 1997). This same polymorphism also increases the risk of sepsis following a burn wound (Barber et al., 2004; O'Keefe et al., 2002). As TNF- $\alpha$ is known to regulate re-epithelisation, it is possible that impaired re-epithelisation increases the risk for both wound infection and IMD (Feiken et al., 1995; Scott et al., 2004).

All of this evidence suggests damage to the nasopharynx and respiratory epithelium increases the likelihood that colonising N. meningitidis can cross the epithelial layer, reach deeper tissues, and cause invasive disease. If colonising $N$. meningitidis are able to prevent rapid repair of microscopic tears in the epithelium or prevent cell migration, one of the important early steps in wound repair, the opportunity to invade deeper tissues and reach the bloodstream is prolonged. Inhibition of host cell wound repair could contribute to an increased probability of IMD. Previous research, using an in vitro model for host cell wound repair, discovered that lowpassage virulent isolates of $N$. meningitidis were indeed effective at inhibiting wound repair, while carriage isolates were more variable, with many not interfering with wound repair at all (Ren \& MacKichan, 2014). This process was shown to require live bacteria undergoing active protein synthesis and was found to be independent of multiple major surface adhesins and antigens that are known to interact with host cells. These included type IV pili, the Opc opacity adhesin, bioactive components of the lipooligosaccharides (i.e., lacto- $N$-neotetraose $\alpha$-chain), and the capsule (Ren \& MacKichan, 2014). 


\subsection{Aims of this thesis}

The overall goal of this thesis was to compare closely related $N$. meningitidis isolates to identify the meningococcal factor responsible for wound repair inhibition by virulent isolates, and investigate how it may contribute to IMD. Unpublished experiments conducted at ESR measured wound repair inhibition by closely related isolates. Those experiments identified one household collection that contained the isolate NZ97/052, obtained from a disease patient, and isolates from asymptomatic healthy household contacts, NZCM111 and NZCM112. These isolates were indistinguishable using standard laboratory typing methods, and were all identified as belonging to the strain type C:2b:P1.5,2 (Simmons et al., 2001). Isolates NZ97/052 and NZCM111 are effective at inhibiting in vitro host cell wound repair, whereas NZCM112 does not inhibit wound repair to the same degree. This thesis focused on this trio of closely-related isolates. In order to determine the bacterial factor responsible, a series of smaller aims were addressed.

Well-characterised in vitro wound repair assays were used to investigate the effect of $N$. meningitidis isolates on host cells, by measuring cell re-epithelialisation (Geiser et al., 2000; Kheradmand et al., 1994). Within the first 24 hours, closure of this area has been shown to be due to cell migration, rather than proliferation (Kheradmand et al., 1994). Cell and bacteria cocultures were supplemented with various nutrients to determine if supplementation affected meningococcal growth or wound repair. These experiments were carried out to determine if bacterial metabolism may play a role in the inhibition of cell migration.

Genome and transcriptome data of isolates belonging to the NZ97/052 household were mined to identify candidate meningococcal genes which may be responsible for the difference in cell migration inhibition. Mutants were made by natural transformation, followed by allelic replacement in the wild-type, with top candidate genes replaced by an antibiotic resistant cassette. The goal was to transform the disease-associated isolate, NZ97/052 into a more carriage-like isolate, by revoking the ability to inhibit wound repair. 
Mutants were analysed using several assays, including bacterial growth assays and in vitro cell migration assays, to test the ability of gene deletion mutants to inhibit host cell wound repair. Gene deletion mutants were compared to the wild-type parent strain, to reveal whether the genes selected played a role in wound repair inhibition by N. meningitidis.

Finally, gene deletion mutants, which differed in their ability to inhibit respiratory epithelial cell migration, were further investigated using antimicrobial assays, growth rate analyses, host cell invasion and adhesion assays, and fluorescence microscopy. The goal of these experiments was to further characterise the deletion mutants and attempt to identify how genes of interest contributed to the inhibition of host cell migration.

\subsection{Applications of this research}

Vaccination can be effective at preventing meningococcal disease. However, multiple factors, including variable vaccine efficacy against serogroup B strains, low vaccination rates, and exclusion of meningococcal vaccinations from routine childhood immunisation, contribute to the persistence of IMD. The meningococcal vaccinations currently in use are still being studied to determine whether they provide long lasting coverage across all age groups. There is currently no universal meningococcal vaccination which effectively targets all strains.

Consequently, vaccine uptake is not very high, and current vaccines are not included on childhood vaccination schedules in many countries. The development of a safe, inexpensive, universal meningococcal vaccine would help to eradicate IMD, and remains a top public health priority. In order to develop improved interventions for meningococcal disease, improving our understanding of the basis of the disease is paramount. With N. meningitidis, vaccines that interrupt asymptomatic carriage have proved to be extremely effective at halting transmission and reducing disease incidence. However, our understanding of host-pathogen interactions during the initiation of invasive disease, carriage, and traversal of the mucosal barrier, is limited. The more that is known about the bacteria and the interaction with the host, the better equipped we can be to prevent onset of disease and treat infections more effectively. Factors 
that distinguish virulent strains, which lead to IMD, from benign strains, which only cause asymptomatic carriage, must be identified.

Clinical isolates that were obtained during the meningococcal epidemic in New Zealand created an extensive library of isolates collected from disease patients, as well as carriage-associated isolates collected from asymptomatic contacts of patients, university students and military recruits. This library of isolates has proved to be extremely useful in investigating characteristics specific to virulent meningococcal strains. Closely related isolates are a useful tool to identify bacterial factors that promote invasive disease. If a bacterial factor associated with virulent meningococci is targeted by vaccination, novel drugs, antibiotics, or antibodies, it can help reduce the damage caused by the bacteria and allow the host to stage an effective immune response in order to clear the infection and recover.

Experiments from this thesis were designed to provide insights into differences between disease-associated and carriage-associated isolates, shed light on how N. meningitidis inhibits wound repair, and identify novel targets for medical intervention or diagnostics. 


\section{Materials and Methods}

\subsection{Bacterial Strains}

\subsubsection{Neisseria meningitidis}

Neisseria meningitidis isolates used for this study were collected and maintained by the Meningococcal Reference Laboratory (MRL) at the Institute of Environmental Science and Research (ESR), as part of the surveillance of meningococcal disease in New Zealand on behalf of the Ministry of Health. The isolates used in this thesis were collected as part of a household contact carriage study, carried out in Auckland, New Zealand in the late 1990s (Simmons et al., 2001). Isolates collected from index patients were cultured from blood or cerebrospinal fluid (CSF) samples. Carriage isolates were collected from household contacts of meningococcal disease patients by nasopharyngeal swab. All isolates were immediately frozen down at $-70^{\circ} \mathrm{C}$ in $15 \% \mathrm{v} / \mathrm{v}$ glycerol following minimal laboratory passage. Frozen working stocks of frequently accessed isolates are maintained to prevent repeated freeze-thaw cycles.

$N$. meningitidis isolates were routinely typed using serological and sequencing methods to determine the serogroup and serosubtype (porA allele). Additional characterisation of isolates, including sequence typing, was performed in some instances. Three of the $N$. meningitidis isolates used in this study were derived from a single household. The NZ97/052 diseaseassociated isolate was derived from a patient with meningococcal disease in 1997; The NZCM111 and NZCM112 isolates were derived from the same household, but were isolated from healthy carriers, and collected as part of the household carriage study (Simmons et al., 2001). These three isolates were indistinguishable by laboratory typing, all were identified as belonging to the strain type C:2b:P1.2,5 (Simmons et al., 2001). The isolates all belonged to clonal complex ST-8. N. meningitidis isolates used in this project are displayed in Table 2.1 
Table 2.1. Meningococcal isolates used in this study.

\begin{tabular}{|c|c|c|c|}
\hline Isolate & Source & $\begin{array}{l}\text { Typing (serogroup: serotype: } \\
\text { serosubtype; sequence type) }\end{array}$ & Reference \\
\hline NZ97/052 & Blood sample & $\mathrm{C}: 2 \mathrm{~b}: \mathrm{P} 1.2,5 ; \mathrm{ST}-8$ & ESR \\
\hline NZCM111 & Nasopharyngeal swab & $\mathrm{C}: 2 \mathrm{~b}: \mathrm{P} 1.2,5 ; \mathrm{ST}-8$ & ESR \\
\hline NZCM112 & Nasopharyngeal swab & $\mathrm{C}: 2 \mathrm{~b}: \mathrm{P} 1.2,5 ; \mathrm{ST}-8$ & ESR \\
\hline NZCM107 & Nasopharyngeal swab & B:nt:19-15; ST-178 & ESR \\
\hline NZCM237 & Nasopharyngeal swab & NG:nt:P1.16; unknown ST & ESR \\
\hline NZCM158 & Nasopharyngeal swab & NG:1:P1.22,14-6,36.2; ST-2154 & ESR \\
\hline NZ97/192 & CSF sample & B:4:P1.7-2,4, ST-41/44 & ESR \\
\hline NZCM238 & Nasopharyngeal swab & B:4:P1.7-2,4, ST-41/44 & ESR \\
\hline NZCM239 & Nasopharyngeal swab & B:4:P1.7-2,4, ST-41/44 & ESR \\
\hline NZCM240 & Nasopharyngeal swab & B:4:P1.7-2,4, ST-41/44 & ESR \\
\hline NZ97/052 $\Delta h p u A$ & $\begin{array}{l}\text { Constructed by gene } \\
\text { deletion }\end{array}$ & $\mathrm{C}: 2 \mathrm{~b}: \mathrm{P} 1.2,5, \mathrm{ST}-8$ & This Study \\
\hline NZ97/052 $\triangle h p u B$ & $\begin{array}{l}\text { Constructed by gene } \\
\text { deletion }\end{array}$ & $\mathrm{C}: 2 \mathrm{~b}: \mathrm{P} 1.2,5, \mathrm{ST}-8$ & This Study \\
\hline NZ97/052 $\triangle h p u A B$ & $\begin{array}{l}\text { Constructed by gene } \\
\text { deletion }\end{array}$ & $\mathrm{C}: 2 \mathrm{~b}: \mathrm{P} 1.2,5, \mathrm{ST}-8$ & This Study \\
\hline $\mathrm{NZ97/052 \Delta fetA}$ & $\begin{array}{l}\text { Constructed by gene } \\
\text { deletion }\end{array}$ & $\mathrm{C}: 2 \mathrm{~b}: \mathrm{P} 1.2,5, \mathrm{ST}-8$ & This Study \\
\hline NZ97/052 $\Delta$ fur & $\begin{array}{l}\text { Constructed by gene } \\
\text { deletion }\end{array}$ & $\mathrm{C}: 2 \mathrm{~b}: \mathrm{P} 1 \cdot 2,5, \mathrm{ST}-8$ & This Study \\
\hline
\end{tabular}




\subsubsection{Growth and storage of $\boldsymbol{N}$. meningitidis}

N. meningitidis isolates were routinely grown on commercially acquired Columbia Blood Agar (CBA) plates (Fort Richard Laboratories, Auckland, New Zealand) at $37^{\circ} \mathrm{C}$ in a humidified $5 \% \mathrm{CO}_{2}$ incubator, for routine passage, or on BBL Brain Heart Infusion (BHI) agar plates (Oxoid, UK), for selective growth with kanamycin. All handling of $N$. meningitidis clinical isolates was carried out following strict biosafety standards; live meningococci were exclusively handled in a Class II biosafety cabinet.

\subsubsection{Making frozen stocks of $\boldsymbol{N}$. meningitidis}

To make frozen stocks, freshly grown N. meningitidis was collected with a sterile cotton swab and resuspended in trypticase soy broth with $15 \%$ glycerol, and frozen at $-80^{\circ} \mathrm{C}$ following minimal laboratory passage.

\subsubsection{N. meningitidis growth from frozen stock}

N. meningitidis was streaked from frozen glycerol stocks and incubated overnight on CBA plates. The next morning, the bacteria from overnight plates were re-streaked onto fresh CBA plates and returned to the incubator for three hours, to ensure the meningococci were in a healthy, exponential phase. The growth from these plates was removed with a sterile cotton swab and resuspended in pre-warmed BHI broth (BD Biosciences, USA) or M199 medium (Thermo Fisher Scientific, USA). One millilitre of this solution was pipetted into a disposable cuvette and optical density (OD) was measured with a Jenway 6300 Visible spectrophotometer. Bacteria were diluted to an $\mathrm{OD}_{600}$ of 0.002 for use in infection experiments, or other optical densities for other experiments (e.g., growth assays). 


\subsubsection{Escherichia coli}

Escherichia coli (E. coli) was used for cloning of a kanamycin resistant cassette and expression of recombinant $N$. meningitidis protein. Specific $E$. coli strains used in this study are displayed in Table 2.2.

Table 2.2. E. coli isolates used in this study

\begin{tabular}{lll}
\hline Isolate & Application & Source \\
\hline BL21(DE3) Hi-Control E. coli & Inducible protein expression & Lucigen \\
& & \\
$\mathrm{DH} 5 \alpha$ E. coli & Cloning & Life Technologies \\
\hline
\end{tabular}

\subsubsection{Growth and storage of $E$. coli}

E. coli was grown on Luria-Bertani (LB) agar at $37^{\circ} \mathrm{C}$ in a humidified $5 \% \mathrm{CO}_{2}$ incubator or in LB broth at $37^{\circ} \mathrm{C}$ at a shaking frequency of $250 \mathrm{rpm}$.

\subsubsection{Antibiotic for selective bacterial growth}

Kanamycin sulfate (Fluka Biochemika, Switzerland) was made up as a 1000x working stock concentration of $50 \mathrm{mg} \mathrm{mL}^{-1}$ in $\mathrm{dH}_{2} \mathrm{O}$ and was filter-sterilised using a $0.22 \mu \mathrm{m}$ filter. This stock was stored at $-20^{\circ} \mathrm{C}$ until needed. LB broth or plates, or $\mathrm{BHI}$ plates were supplemented with 50 $\mu \mathrm{g} / \mathrm{mL}$ kanamycin where required. 


\subsection{Tissue cell culture}

The human bronchial epithelial cell line 16HBE14o- (abbreviated as 16HBE) is comprised of airway bronchial epithelial cells that have been immortalised with origin of replicationdefective SV40 (Cozens et al., 1994). This transformed cell line may be differentiated to form polarized cell layers in vitro, providing an opportunity to develop an epithelial cell culture model. 16HBE cells were chosen due to their formation of tight junctions between cells. Tight junctions are an important trait, as this project focused on how $N$. meningitidis penetrates the epithelial layer to cause invasive disease. 16HBE cells were used for all experiments to investigate interactions between $N$. meningitidis and host cells. All cell culture was carried out using aseptic technique in a cell culture hood.

\subsubsection{Recovery of 16HBE cells from frozen stocks}

Frozen vials of $16 \mathrm{HBE}$ cells were removed from liquid nitrogen storage and thawed quickly in warm water. A frozen stock was pipetted into a T-25 flask with $9 \mathrm{~mL}$ of GIBCO Medium M199 (Thermo Fisher Scientific, USA), supplemented with 10\% heat-inactivated Foetal Bovine Serum (FBS). Flasks were incubated at $37^{\circ} \mathrm{C}$ and medium was replaced every two days until cells were confluent.

\subsubsection{Passage of $16 \mathrm{HBE}$ cells}

Cells were grown in $25 \mathrm{~mL}$ of M199 medium (Thermo Fisher Scientific, USA) supplemented with $10 \%$ FBS in a T75 flask (Sigma-Aldrich, Missouri, USA) and incubated at $37^{\circ} \mathrm{C}, 5 \% \mathrm{CO}_{2}$. When $16 \mathrm{HBE}$ cells were passaged, medium was aspirated and $10 \mathrm{~mL}$ pre-warmed phosphate-buffered saline (PBS) was added to the wells, and cells were incubated at $37^{\circ} \mathrm{C}$ for 10 minutes. PBS was then removed and $1.5 \mathrm{~mL}$ of trypsin-EDTA was added. The flasks were incubated for 20-30 minutes at $37^{\circ} \mathrm{C}$. Ten millilitres of M199 medium with $10 \%$ FBS was added and cells were pipetted up and down 50 times to break up tight junctions. Cells were counted by pipetting 10 
$\mu \mathrm{L}$ of cell suspension onto the grid of a haemocytometer. This count was then used to calculate the volume of cell suspension needed to further passage or split cells.

\subsubsection{Making frozen stocks of cells}

To make liquid nitrogen stocks of $16 \mathrm{HBE}$, cells were passaged as described in section 2.2.2. After trypsin-EDTA lifted the cells from the T-75 flask, the suspension was spun at $1000 \mathrm{rpm}$ for 10 minutes. The resulting pellet was gently resuspended in $4.5 \mathrm{~mL} F B S$ and $0.5 \mathrm{~mL}$ dimethyl sulfoxide (DMSO). The cell suspension was aliquoted into $1 \mathrm{~mL}$ cryotubes and frozen using an isopropanol chamber at $-80^{\circ} \mathrm{C}$ for approximately two hours. When cells were frozen, tubes were transferred to liquid nitrogen.

\subsubsection{Growing confluent cells for assays}

16HBE cells were cultured onto 24 well plates for bacterial growth assays, invasion assays, adhesion assays, and in vitro scratch assays. Five hundred microlitres of cell suspension at $6 \times 10^{4}$ cells $/ \mathrm{mL}$ was added to each well in the plate, and cells were grown for two days to reach confluence.

\subsubsection{Culturing 16HBE cells onto chamber slides for fluorescence microscopy}

16HBE cells were seeded onto Nunc Lab-Tek II eight-well chamber slide system (Thermo Fisher Scientific, USA). Each chamber well had $450 \mu \mathrm{L}$ of cell suspension at $1.2 \times 10^{5}$ cells $/ \mathrm{mL}$ added and cells were grown for 24 hours, until confluent.

\subsubsection{Culturing 16HBE cells onto Oris cell migration plates}

Collagen I coated Oris ${ }^{\mathrm{TM}}$ Cell Migration Assay plates (Platypus Technologies Madison, WI) were warmed to room temperature prior to the addition of cell suspension, and cell seeding stoppers were pressed securely in place with tweezers that had been sterilised with ethanol. One hundred microlitres of suspended $16 \mathrm{HBE}$ cells at a density of $3 \times 10^{5}$ cells $/ \mathrm{mL}$ were added to each 
well of the 96 well Oris plate. Plates were incubated at $37^{\circ} \mathrm{C}, 5 \% \mathrm{CO}_{2}$ for 24 hours to permit cell attachment.

\subsubsection{Culture of polarised 16HBE cells}

A Corning Transwell system (Sigma-Aldrich, Missouri, USA) was used to investigate bacterial attachment to fully polarised $16 \mathrm{HBE}$ cells. Pore permeable supports $(0.4 \mu \mathrm{m})$ were warmed at $37^{\circ} \mathrm{C}$ for one hour in M199 medium prior to seeding cells, in order to warm the membrane. After this incubation, $600 \mu \mathrm{L}$ of M199 medium with 10\% FBS was added to the basal well. $16 \mathrm{HBE}$ cells were seeded at $10^{4}$ cells $/ \mathrm{cm}^{2}$, with $100 \mu \mathrm{L}$ of cell suspension added to the apical well. Transwells were incubated overnight at $37^{\circ} \mathrm{C}$ to allow attachment of $16 \mathrm{HBE}$ cells to the Transwell membrane. The next day, when cells were attached and confluent, the apical well supernatant was removed. The membranes were washed three times with PBS, then M199 medium (without FBS) was added back to the apical chamber. For three weeks apical and basal medium was replaced daily, to allow for full polarisation of $16 \mathrm{HBE}$ cells. To determine the permeability of cells on Transwell filters, Transwell Inserts were washed with pre-warmed PBS before adding $500 \mu \mathrm{L}$ of Evans blue $(0.67 \mathrm{mg} / \mathrm{ml}$ ) (Sigma-Aldrich, Missouri, USA), diluted in M199 medium with 10\% FBS, to the apical chamber. M199 medium with $10 \%$ FBS was added to the basal chamber. After 10 minutes, $\mathrm{OD}_{600}$ was measured in the lower chamber.

\subsubsection{Infection experiments}

Prior to initiating infection, confluent cells were washed with PBS and incubated in serum-free M199 medium for 24 hours to synchronise cells to same cell cycle phase. A visible spectrophotometer was used to dilute bacteria to an appropriate $\mathrm{OD}_{600}$, in order to infect with bacteria at a molarity of infection (MOI) of 10. For certain migration assays, growth assays, and association assays, $16 \mathrm{HBE}$ cell and bacteria co-culture were supplemented with various metal ions using solutions of $\mathrm{FeSO}_{4}\left(\mathrm{Fe}^{2+}\right), \mathrm{FeCl}_{3}\left(\mathrm{Fe}^{3+}\right)$, and $\mathrm{ZnSO}_{4}$ at various concentrations. Each supplement was made by combining crystals with $\mathrm{dH}_{2} \mathrm{O}$ to a $1 \mathrm{M}$ concentration and sterilising with a $0.22 \mu \mathrm{m}$ filter. Each solution was diluted as needed and made fresh for each experiment. 
Pooled non-essential amino acids (NEAA) were also used for supplementation experiments, and this included glycine, L-alanine, L-asparagine, L-aspartic acid, L-glutamic acid, L-proline, and Lserine (Thermo Fisher Scientific, USA).

\subsection{DNA and RNA purification}

\subsubsection{Plasmids}

Plasmids used in this study are listed in Table 2.3 along with relevant properties

Table 2.3. Plasmids used in this study

\begin{tabular}{lll}
\hline Plasmid & Properties & Reference \\
\hline pUC19 & Cloning vector, Amp ${ }^{\mathrm{R}}$ & (Norrander et al., 1983) \\
pET28a & Expression vector, $\mathrm{Kan}^{\mathrm{R}}$, IPTG inducible & Novagen \\
\hline
\end{tabular}

\subsubsection{Plasmid mini preparation}

E. coli, containing the pUC19 plasmid with the aphA3 kanamycin resistance gene cloned into its unique EcoRI restriction site, was transferred from frozen glycerol stocks into $3 \mathrm{~mL}$ LB broth, supplemented with $50 \mu \mathrm{g} / \mathrm{mL}$ kanamycin. Bacteria were grown at $37^{\circ} \mathrm{C}$ overnight on a shaking incubator. Zyppy ${ }^{\text {TM }}$ Plasmid Miniprep Kit (Zymo Research, California, USA) was used to isolate the plasmid, according to the manufacturer's instructions. Plasmid DNA was eluted in $30 \mu \mathrm{L}$ Elution Buffer and stored at $-20^{\circ} \mathrm{C}$.

\subsubsection{Genomic DNA isolation}

N. meningitidis was grown overnight on CBA plates for 14 hours. Overnight growth was swabbed directly from the plates into $300 \mu \mathrm{L}$ Cell Lysis Solution (QIAGEN, Venlo, Netherlands), and heat killed for 60 minutes at $56^{\circ} \mathrm{C}$. Samples were pipetted up and down and incubated at $80^{\circ} \mathrm{C}$ for five minutes to fully lyse the cells. DNA was purified from lysed Gram-negative bacteria using Gentra Puregene Yeast/Bact. Kit (QIAGEN, Venlo, Netherlands), according to the 
manufacturer's instructions. Genomic DNA was resuspended in $100 \mu \mathrm{L}$ DNA hydration solution and incubated for $65^{\circ} \mathrm{C}$ for one hour to dissolve DNA, stored at $-20^{\circ} \mathrm{C}$.

\subsubsection{RNA isolation}

Mid-log phase N. meningitidis were added to $16 \mathrm{HBE}$ cells, to stimulate expression of proteins involved in host interactions. Fresh $N$. meningitidis, swabbed from plates, was resuspended at an $\mathrm{OD}_{600}$ of 0.25 in M199 medium, with 10\% FBS. This bacterial suspension was added to confluent $16 \mathrm{HBE}$ cells in a 24-well plate. The cell culture medium was removed from each well and replaced with $1 \mathrm{ml}$ of bacterial suspension. After two hours of incubation at $37^{\circ} \mathrm{C}, 900 \mu \mathrm{L}$ of bacterial solution was combined with $100 \mu \mathrm{l}$ of 10X "Stop Solution" (5\% buffer-equilibrated phenol [pH 7.4] in ethanol) (Bernstein et al., 2002). The total RNA was purified as described by Gaynor et al (2004). Briefly, tubes were centrifuged for five minutes to pellet cells. The supernatant was removed and discarded, and pellets were frozen, at least overnight, at $-80^{\circ} \mathrm{C}$. The bacterial pellets were briefly thawed and resuspended in $50 \mu \mathrm{L}$ of $0.4 \mathrm{mg} / \mathrm{mL}$ lysozyme in TE and incubated at room temperature for five minutes. Tubes had $950 \mu \mathrm{L}$ of TRIzol reagent (Thermo Fisher Scientific, USA) added to lyse the cells. The tubes were capped and vortexed for 30 seconds, then allowed to sit at room temperature for five minutes. Next $200 \mu \mathrm{L}$ of chloroform was added; after capping the tubes, they were shaken vigorously by hand for 15 seconds and incubated at room temperature for three minutes. Samples were centrifuged at $12,000 \times g$ for 15 minutes. The top phase was then combined with an equivalent volume of $70 \%$ ethanol in a new tube. This solution was applied to an RNEasy Mini column (QIAGEN, Venlo, Netherlands) and washed according to the manufacturer's instructions. On-column RNAse-free DNAse treatment (QIAGEN, Venlo, Netherlands) was used to digest any remaining DNA. The concentration and quality of RNA was assessed with a 2100 Bioanalyzer Instrument (Agilent Technologies, California, USA). Double stranded cDNA was generated using SuperScript DoubleStranded cDNA Synthesis Kit (Thermo Fisher Scientific, USA) with $2 \mu \mathrm{g}$ of bacterial RNA and a random hexamer primer. 


\subsection{PCR and Sequencing}

\subsubsection{Primers}

Primers were designed based on the N. meningitidis strain NMBG2136 (Budroni et al., 2011) sequence from the NCBI database. This serogroup B strain belongs to the same hypervirulent clonal complex (CC-8) as the NZ97/052 isolate at ESR, although they belong to different serogroups. Primer3 software (Rozen \& Skaletsky, 2000) was used to design the primers and Oligoanalyser 3.1 (Integrated DNA Technologies, Singapore) was used to check the primers for hairpins, self-dimers, and hetero-dimers. NCBI Basic Local Alignment Search Tool (BLAST) (Altschul et al., 1990) was used to check for any possible binding of the primers to sequences outside the gene regions. Primers were designed such that the outermost primers were tagged with unique restriction sites that were not present in the genes to be deleted. This was done in case the PCR product needed to be cloned into a plasmid, such as pUC19. The inner primers contained sequences that overlapped with the primers that amplify the aphA3 kanamycin resistance gene (Table 2.4). Primers were synthesised by Integrated DNA Technologies ${ }^{\text {TM }}$ (IDT; Singapore) and were provided lyophilised. Primers were suspended in $\mathrm{dH}_{2} \mathrm{O}$ to a concentration of $100 \mu \mathrm{M}$. Working stocks were diluted to a final concentration of $10 \mu \mathrm{M}$.

Table 2.4. Oligonucleotide primers used for mutagenesis

\begin{tabular}{clll}
\hline Gene & Primer name & Nucleotide sequence (5'-3') & Features \\
\hline hpuA & HpuA_Xbal_Fw_1 & GCGTCTAGACAAAGCCCTGTCCTTACTGC & $\underline{\text { Xbal site }}$ \\
\cline { 2 - 4 } & HpuA_Kan_Rv_2 & GCTGGTTCAGACGGCGAATTCCGCCGGATGTGT & Anneals to Km1; \\
& TTCCAGTGTTG & $\underline{\text { EcoR1 site }}$ \\
\cline { 2 - 4 } & HpuA_Kan_Fw_3 & AGATGTCTAAAAAGCGAATTCCGCTATCACCGC & Anneals to Km2; \\
& HpuA_BamHI_Rv_4 & GCGGGATCC CTTCCAATTTGCCGTTTTTG & $\underline{\text { EcoR1 site }}$ \\
\cline { 2 - 4 } & & & BamHI site
\end{tabular}




\begin{tabular}{|c|c|c|c|}
\hline Gene & Primer name & Nucleotide sequence (5'-3') & Features \\
\hline \multirow[t]{6}{*}{ hpuB } & HpuB_Sacl_Fw_1 & GCGGAGCTCATCCCCTTCAAACCCGTATT & Sacl site \\
\hline & \multirow[t]{2}{*}{ HpuB_Kan_Rv_2 } & GCTGGTTCAGACGGCGAATTCCGCTGTTCGTCG & Anneals to $\mathrm{Km} 1$; \\
\hline & & TTGACCAAGAG & EcoR1 site \\
\hline & \multirow[t]{2}{*}{ HpuB_Kan_Fw_3 } & AGATGTCTAAAAAGCGAATTCCGCGCAAGGCC & Anneals to Km2; \\
\hline & & TATACGCTGTTC & EcoR1 site \\
\hline & HpuB_Sphl_Rv_4 & GCGGCATGCGAGGTAAAGCGTTGGATGCC & Sphl site \\
\hline \multirow[t]{6}{*}{ fetA } & FetA_Scal_Fw_1 & GCGAGTACTAGGACGAACAAATGAACGCC & Scal site \\
\hline & \multirow[t]{2}{*}{ FetA_Kan_Rv_2 } & GCTGGTTCAGACGGCGAATTCCGCATATCGGTT & Anneals to $\mathrm{Km} 1$; \\
\hline & & GCGGTGCTTTC & EcoR1 site \\
\hline & \multirow[t]{2}{*}{ FetA_Kan_Fw_3 } & AGATGTCTAAAAAGCGAATTCCGCGTGGGTTCG & Anneals to $\mathrm{Km} 2$; \\
\hline & & ATATTGGTGGC & EcoR1 site \\
\hline & FetA_Sphl_Rv_4 & GCGGCATGCGCTGTGCGGATAGTAGAACG & Sphl site \\
\hline \multirow[t]{6}{*}{ fur } & Fur_Sacl_Fw_1 & GCGAGTACTCACCCTGTCTGTGTCCGAC & $\underline{\text { Sacl site }}$ \\
\hline & \multirow[t]{2}{*}{ Fur_Kan_Rv_2 } & GCTGGTTCAGACGGCGAATTCCGCGCCGGTAA & Anneals to Km1; \\
\hline & & CTTTCAGTCCAC & EcoR1 site \\
\hline & \multirow[t]{2}{*}{ Fur_Kan_Fw_3 } & AGATGTCTAAAAAGCGAATTCCGCACGCGCTTT & Anneals to $\mathrm{Km} 2$; \\
\hline & & ATATGTACGGC & EcoR1 site \\
\hline & Fur_XBal_Rv_4 & GCGTCTAGAATAGGCAGGATCGGGAAAGG & Xbal site \\
\hline \multirow[t]{3}{*}{ aphA3 } & \multirow[t]{2}{*}{ Km_Fw_1 } & GCGGAATTCGCCGTCTGAACCAGCGAACCATTT & EcoRI site \\
\hline & & GAGG & \\
\hline & Km_Rv_1 & GCGGAATTCGCTTTTTAGACATCTAAATCTAGG & EcoRI site \\
\hline
\end{tabular}




\subsubsection{Polymerase chain reaction}

Advantage $^{\circledR} 2$ PCR Kit (Clontech, California, USA) was used for the polymerase chain reaction (PCR). A reaction mixture was prepared in a $200 \mu \mathrm{L}$ tube on ice, containing $2.5 \mu \mathrm{L}$ PCR buffer, 0.5 $\mu \mathrm{L}$ dNTP mix, $0.5 \mu \mathrm{L}$ polymerase mix, $1 \mu \mathrm{L}$ DNA template, oligonucleotide primers to a final concentration of $0.2 \mathrm{mM}$ each, and PCR-grade $\mathrm{H}_{2} \mathrm{O}$ to a total volume of $25 \mu \mathrm{L}$. PCR was carried out in ProFlex ${ }^{\mathrm{TM}}$ 96-well PCR System (Thermo Fisher Scientific, USA). Cycle parameters were dependent on target product size and are listed in Table 2.5 and Table 2.6.

\subsubsection{PCR cycle parameters}

Table 2.5. PCR cycle parameters for target size $<1 \mathrm{~kb}$

\begin{tabular}{lll}
\hline Number of cycles & Temperature & Duration \\
\hline 1 cycle & $95^{\circ} \mathrm{C}$ & 60 seconds \\
35 cycles & $95^{\circ} \mathrm{C}$ & 30 seconds \\
& $68^{\circ} \mathrm{C}$ & 60 seconds \\
1 cycle & $68^{\circ} \mathrm{C}$ & 60 seconds \\
1 cycle & $4^{\circ} \mathrm{C}$ & Indefinitely \\
\hline
\end{tabular}

Table 2.6. PCR cycle parameters for target size $1-5 \mathrm{~kb}$

\begin{tabular}{lll}
\hline Number of cycles & Temperature & Duration \\
\hline 1 cycle & $95^{\circ} \mathrm{C}$ & 60 seconds \\
35 cycles & $95^{\circ} \mathrm{C}$ & 30 seconds \\
& $62^{\circ} \mathrm{C}$ & 30 seconds \\
& $68^{\circ} \mathrm{C}$ & 3 minutes \\
1 cycle & $68^{\circ} \mathrm{C}$ & 60 seconds \\
1 cycle & $4^{\circ} \mathrm{C}$ & Indefinitely \\
\hline
\end{tabular}




\subsubsection{DNA gel electrophoresis}

$\mathrm{E}-G e l^{\circledR} 2 \%$ agarose gels (Thermo Fisher Scientific, USA) were used to perform the analysis of the products of PCR. Each well was loaded with $5 \mu \mathrm{L}$ PCR product and $15 \mu \mathrm{L} d \mathrm{H}_{2} \mathrm{O}$. O'RangeRuler

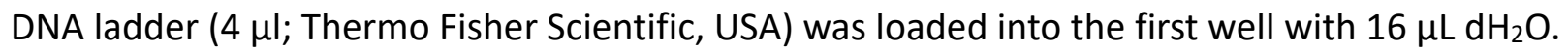
The DNA ladder used was either $100 \mathrm{bp}$ or $200 \mathrm{bp}$, as specified in each figure in the results section. Electrophoresis was run in an E-Gel ${ }^{\circledR}$ Base (Thermo Fisher Scientific, USA) for 15 minutes. Gels were analysed by Gel Doc ${ }^{\mathrm{TM}}$ XR+ System (Bio-Rad, USA). Images were inverted using ImageJ (Schneider et al., 2012).

\subsubsection{DNA purification}

PCR products were purified using the DNA Clean \& Concentrator ${ }^{\mathrm{TM}}-5$ kit (Zymo Research, California, USA) according to the manufacturer's instructions and DNA was eluted in $6 \mu \mathrm{L} \mathrm{dH2O}$ and stored at $-20^{\circ} \mathrm{C}$.

\subsection{Natural transformation and mutagenesis}

\subsubsection{Construction of mutagenic PCR fragments}

N. meningitidis NZ97/052 genomic DNA was used as a template to amplify about 200 bp of flanking regions of each target gene ( $h p u A, h p u B, f e t A$, or fur). The aphA3 gene was amplified from pUC19 AphA3 plasmid (kindly provided by Assoc Prof Charlene Kahler, University of Western Australia, Perth). PCR products were checked by agarose gel and, if the correct size, purified (see section 2.4.3) and used to set up a fusion PCR reaction. One microlitre of purified PCR product from each flanking region of the target gene was combined with $1 \mu \mathrm{L}$ of the purified aphA3 PCR product, and the three products together formed an internally-priming DNA template for a fusion PCR reaction. The fusion PCR product was checked on an agarose gel to confirm that it was the right size, then purified. 
Once successful fusion of the PCR products had been confirmed, the final product was used as a template in multiple replicate PCR reactions to generate more product for mutagenesis. These replicate PCR reactions were combined and purified with the DNA Clean \& Concentrator-5 kit (Zymo Research, California, USA), to have sufficiently concentrated DNA for natural

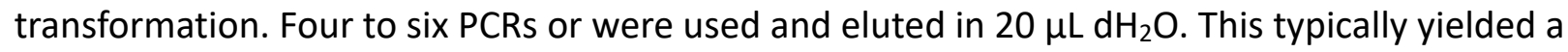
concentration of about $350 \mathrm{ng} / \mu \mathrm{L}$.

\subsubsection{Natural transformation for mutagenesis of $\boldsymbol{N}$. meningitidis.}

Natural transformations of $N$. meningitidis were performed using purified, concentrated PCR products containing homologous regions flanking the target gene, on either side of the kanamycin resistance cassette, aphA3. N. meningitidis isolate NZ97/052 was streaked onto room temperature CBA plates from $-80^{\circ} \mathrm{C}$ freezer glycerol stocks and grown overnight in a $37^{\circ} \mathrm{C}$ humidified $\mathrm{CO}_{2}$ incubator. Bacteria were re-streaked the next morning onto fresh CBA plates and left to grow for three hours. $N$. meningitidis growth was gathered with an inoculating loop and plated in a circular shape $\sim 1 \mathrm{~cm}$ in diameter on a fresh CBA plate. Ten microlitres of highly concentrated purified fusion PCR DNA (typically about 3.50 $\mu \mathrm{g}$ DNA) was pipetted onto this circle of bacteria and mixed with an inoculating loop. Plates were incubated upright at $37^{\circ} \mathrm{C}$ for five hours to allow for DNA uptake by natural transformation. After incubation, $N$. meningitidis was gathered with a swab and resuspended in $150 \mu \mathrm{L} \mathrm{BHI}$ broth. One hundred microlitres of the bacterial suspension was spread onto a BHI agar plate supplemented with $50 \mu \mathrm{g} / \mathrm{mL}$ kanamycin, and $50 \mu \mathrm{L}$ were spread onto a second plate. These plates were incubated overnight at $37^{\circ} \mathrm{C}$ with $5 \% \mathrm{CO}_{2}$. The next morning six individual colonies for each mutagenesis were selected, and each was re-streaked onto a fresh kanamycin $\mathrm{BHI}$ plate and incubated overnight at $37^{\circ} \mathrm{C}$. The following morning, half of the bacteria from each plate was harvested for a glycerol stock and stored in the $-80^{\circ} \mathrm{C}$ freezer; the remaining bacteria were used for genomic DNA purification (Section 2.3.3) using Gentra Puregene Yeast/Bact. Kit (QIAGEN, Venlo, Netherlands). 


\subsubsection{Validation of gene deletion mutants}

PCR was used to validate mutants and verify successful deletion of the targeted gene. Genomic DNA was isolated from the mutagenic isolates (Section 2.3.3). One microlitre of genomic DNA was used for PCR, and a reaction was completed with genomic DNA from the wild-type NZ97/052 parent isolate as a template. To amplify the gene region, the outer primers (e.g. those ending with F_1 and R_4) were used (Table 2.4). Mutants were confirmed by checking the product size on a $2 \%$ Agarose E-gel, compared with the wild-type parent. In each case, the wildtype sequence was a different size from the mutagenic sequence with inserted aphA3, resulting in different band sizes on the agarose gel.

\subsubsection{DNA sequencing}

PCR products from the mutants were also sequenced to further confirm that the aphA3 kanamycin resistance cassette was incorporated into the chromosome at the site expected. DNA was sequenced using in-house Sanger sequencing at ESR. The 4Peaks software (Griekspoor \& Groothuis, 2005) was used to analyse the sequencing chromatogram. NCBI BLAST (Altschul et al., 1990) search was used to compare the gene region from the mutants to the predicted sequence of kanamycin resistance aphA3 gene.

\subsubsection{Heterologous expression of HpuA in E. coli}

\subsubsection{Transformation of E. coli BL21}

To heterologously complement $E$. coli with the $N$. meningitidis $h p u A$ gene, the $h p u A$ sequence from the NMBG2136 isolate (Budroni et al., 2011) was downloaded using NCBI gene. The coding region was converted to a codon-optimised sequence for expression in E. coli, using the Codon Optimisation Tool (IDTDNA.com). The predicted amino acid sequence was checked to confirm that it was not altered from that of NMBG2136; this also had the added benefit of enabling removal of the homopolymeric G-tract in hpuA that is a frequent site of slipped-strand mispairing and might contribute to plasmid instability in E. coli. The codon optimised hpuA sequence contains a His-tag and a linker sequence at the $\mathrm{N}$-terminus, however, this was not 
predicted to alter the function. Using this sequence, a plasmid construct of the synthetic $h p u A$ gene, cloned into the Ndel and Xhol sites of the pET28a(+) expression vector, was ordered from Twist Bioscience. The ATG start codon for hpuA was incorporated into the ATG of the Ndel restriction site to ensure proper orientation for the T7lac promoter. The stop codon was also removed, to add a His-tag to the protein and enable purification for future experiments. Vector constructs were resuspended in TE to a final concentration of $50 \mathrm{ng} / \mu \mathrm{l}$. One microlitre (50 $\mathrm{ng}$ ) was used to transform BL21 (DE3) Hi-Control cells (Lucigen), according to the manufacturer's protocol. Hi-Control cells were thawed on wet ice and transferred to a pre-chilled $15 \mathrm{~mL}$ culture tube. One microlitre (50 ng) of DNA was added to the cells, which were stirred with a pipette tip. The culture tube, containing cells and DNA, was incubated on ice for 30 minutes. Cells were heat shocked by placing the tube in a $42^{\circ} \mathrm{C}$ water bath for 45 seconds, then returned to ice for two minutes, followed by addition of $960 \mu \mathrm{L}$ of the provided room temperature Recovery Medium. The resuspended cells were placed in a shaking incubator at $250 \mathrm{rpm}$ for one hour at $37^{\circ} \mathrm{C}$. Fifty microlitres of the transformed cells were plated on LB agar containing $50 \mu \mathrm{g} / \mathrm{mL}$ kanamycin and incubated overnight at $37^{\circ} \mathrm{C}$. One colony was picked, grown overnight, and used for glycerol stocks and subsequent experiments.

\subsubsection{Induction of HpuA}

A single colony was picked from an overnight plate of $E$. coli containing the $h p u A$ pET28a plasmid. The colony was grown in $5 \mathrm{~mL}$ of kanamycin LB broth at $37^{\circ} \mathrm{C}$ with shaking, until the culture reached an $\mathrm{OD}_{600}$ of 0.6 . This starter culture was split into two $50 \mathrm{~mL}$ conical tubes. The expression of HpuA was induced with 0.4 mM IPTG in one tube and repressed with $10 \mathrm{~g} / \mathrm{L}$ glucose in the other tube. Cultures were incubated at $37^{\circ} \mathrm{C}$ with shaking. Heterologous expression of HpuA was confirmed by protein gel analysis. Impact of HpuA on E. coli growth rate was monitored by optical density of induced and uninduced cultures; $50 \mu \mathrm{L}$ of culture was removed every hour to measure optical density. The experiment was performed in triplicate, two independent times. 


\subsubsection{Confirmation of HpuA expression by protein gel analysis}

\subsubsection{Total cell protein fraction}

The total cell protein fraction was collected for induced and uninduced HpuA-expressing $E$. coli at zero, one, three, and five hours. Optical density was used to normalise cell loading volume of samples. A $1 \mathrm{~mL}$ sample of each culture was centrifuged at $10,000 \times \mathrm{g}$ for one minute. The supernatant was discarded, and the cell pellet was drained by inverting the tube onto a paper towel. The pellet was resuspended in $100 \mu \mathrm{L}$ of $1 \mathrm{X}$ PBS, giving a concentration factor of 10 -fold. One hundred microlitres of $2 X$ sodium dodecyl sulfate (SDS) sample buffer (Thermo Fisher Scientific, USA) was added and sample was passed through a 27-gauge needle several times to reduce viscosity. The sample was heated for three minutes at $85^{\circ} \mathrm{C}$ and then stored at $-20^{\circ} \mathrm{C}$ until use in Sodium dodecyl sulfate polyacrylamide gel electrophoresis (SDS-PAGE) analysis.

\subsubsection{Preparation of reagents for SDS-PAGE}

PBS

1X Phosphate Buffered Saline was made up by dissolving PBS tablets (Sigma-Aldrich, Missouri, USA) in $\mathrm{dH}_{2} \mathrm{O}$. PBS was sterilised by autoclaving at $121^{\circ} \mathrm{C}$ for 15 minutes.

\section{Tris-Glycine SDS Running buffer}

10X Tris-Glycine SDS Running Buffer (25 mM Tris Base, 192 mM Glycine, 0.1\% SDS) was

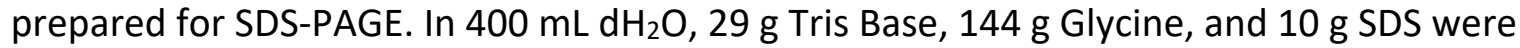
dissolved with assistance of a magnetic stir bar. Once reagents were dissolved, the volume was adjusted to $1000 \mathrm{~mL}$ with $\mathrm{dH}_{2} \mathrm{O}$. Before electrophoresis, buffer was diluted to $1 \mathrm{X}$ with $\mathrm{dH}_{2} \mathrm{O}$. 


\subsubsection{SDS Page}

A prepared Novex 10-20\% Tris-Glycine mini wedge well gel (Thermo Fisher Scientific, USA) was used for SDS-PAGE. To prepare the mini gel, the comb was removed, and gel wells were washed three times with running buffer. Each well was loaded with $40 \mu \mathrm{L}$ of total cell pellet fraction combined with sample buffer. One well was loaded with $10 \mu \mathrm{L}$ SeeBlue Plus2 Pre-Stained protein standard (Thermo Fisher Scientific, USA) as a size reference. The gel was run at $225 \mathrm{~V}$ constant voltage for 45 minutes.

\subsubsection{Staining SDS Page}

After running, the mini gel was removed from the cassette and washed three times for five minutes in $200 \mathrm{~mL} \mathrm{dH}{ }_{2} \mathrm{O}$. The gel was then stained in $50 \mathrm{~mL}$ Bio-Safe Coomassie G-250 stain (Bio-Rad, USA) for one hour, with gentle rocking. The gel was rinsed with $\mathrm{dH}_{2} \mathrm{O}$ to remove the stain and was de-stained overnight in $200 \mathrm{~mL} \mathrm{dH_{2 }}$ O. The gel was photographed and the image then analysed by ImageJ software (Schneider et al., 2012).

\subsection{Cell Migration assays}

\subsubsection{Oris cell migration assay}

Collagen I coated Oris ${ }^{\mathrm{TM}}$ Cell Migration Assays (Platypus Technologies, Madison, WI) were used to detect cell migration. Each well in the Oris cell migration plates contains a flexible plastic stopper insert to prevent cells from adhering to a small section in the centre of the well. When the plates had been seeded with $16 \mathrm{HBE}$ cells and incubated for 24 hours (Section 2.2.4.2), all stoppers were removed from the wells using a sterile Oris Stopper Tool. The stoppers were left in place for 8-10 wells, which were retained until the results were read. The cell-free area from these wells was used as a proxy for the initial wound area. 16HBE cells were infected by adding $14 \mu \mathrm{L}$ of a freshly-made $N$. meningitidis $\mathrm{M} 199$ medium suspension (at $\mathrm{OD}_{600}=0.002$ ) to each well, resulting in an approximate $\mathrm{MOI}$ of 10 . Plates were covered with a Breathe-Easy sealing membrane (Sigma-Aldrich, Missouri, USA) to prevent internal contamination and incubated for 
16 hours at $37^{\circ} \mathrm{C}$ and $5 \% \mathrm{CO}_{2}$. Following 16 hours of incubation of $16 \mathrm{HBE}$ cells with $\mathrm{N}$. meningitidis, the Breathe-Easy membrane was peeled off the plate in the Class II cabinet. Stoppers were removed from the reference wells and medium was aspirated from all 96 wells using a multichannel pipette. Cells were washed twice with $100 \mu \mathrm{L}$ pre-warmed PBS, which was then completely removed. The cells were fixed with $100 \mu \mathrm{L}$ ice cold methanol per well for three minutes. The methanol was then entirely aspirated, and wells were air dried. External surfaces of the Oris plates were disinfected with $70 \%$ ethanol and sealed inside a zip lock bag before the plate was removed from the biosafety cabinet and examined under the microscope. Each well was imaged and photographed using an Olympus IX71 inverted microscope on 4X magnification. ImageJ software (Schneider et al., 2012) was used to trace the outline of and measure the wound area. The remaining wound area was calculated as a percentage of the area of the wells that had not had their stoppers removed. P-value was calculated in a One-Way analysis of variance (ANOVA), using SAS (2013), to determine significant difference in wound repair from the wild-type disease isolate NZ97/052.

\subsubsection{In vitro wound repair scratch assay}

16HBE cells were grown to $90 \%$ confluence in a 24 -well plate, then a single scratch was made carefully down the middle of each well using a sterile $20 \mu \mathrm{L}$ pipette tip. The cells were washed three times with warm PBS to remove any cellular debris, and $1 \mathrm{~mL}$ of fresh M199 medium with $10 \%$ FBS was added back to the wells. The plates were returned to the incubator for two hours, after which each well was photographed with a $4 \mathrm{X}$ objective lens, using an inverted microscope (Olympus), prior to the addition of bacteria. These images represented the initial wound area and were used to calculate the final extent of wound repair. The wound area that was photographed was marked with permanent marker on the underside of the plate. Cells were infected with $N$. meningitidis, at an approximate $\mathrm{MOI}$ of 10 , and plates were covered with a Breathe-Easy sealing membrane (Sigma-Aldrich, Missouri, USA) and incubated for 16 hours at $37^{\circ} \mathrm{C}$ and $5 \% \mathrm{CO}_{2}$. The following day the cell culture supernatants were aspirated and discarded. The cells were washed gently once with $1 \mathrm{~mL}$ of warm PBS and fixed with $1 \mathrm{~mL}$ of ice-cold methanol for three minutes. The methanol was aspirated, and the plate was air-dried for five 
minutes. The wells were again photographed in the identical area with an inverted microscope (Olympus), and the same wound area was measured with ImageJ software (Schneider et al., 2012); the degree of wound closure was calculated as a percentage of the initial wound area. SAS (2013) was used to calculate significance using a one-way ANOVA.

\subsection{Growth rate analysis}

\subsubsection{Growth curve assay}

Growth of meningococcal isolates was measured using the optical density of bacteria grown in liquid cultures over a time course. $N$. meningitidis was grown overnight on CBA plates, then collected with a sterile cotton swab and resuspended in either BHI broth (Oxoid), or M199 medium with $10 \%$ inactivated FBS. Bacteria were diluted to an $\mathrm{OD}_{600}$ of 0.2 . Fifteen millilitres of this suspension were then added to an empty T-75 flask (Sigma-Aldrich, Missouri, USA), along with nutritional supplementation as required (e.g. $\mathrm{FeSO}_{4}, \mathrm{FeCl}_{3}, \mathrm{ZnSO}_{4}$, or NEAA). Flasks were placed flat in the incubator, to prevent oxygen limitation in liquid culture. Every hour, cultures were mixed by pipetting up and down and $50 \mu \mathrm{L}$ of culture was removed to measure optical density. Measurements were taken in triplicate and the experiment was performed three separate times.

\subsubsection{Counting viable colony forming units}

Growth of meningococcal isolates was measured by enumeration of colony forming units (CFU) of bacteria grown in liquid medium. To mimic the conditions of the Oris cell migration assay, $16 \mathrm{HBE}$ cells were cultured in 24 well plates until confluent. Prior to infection, the $16 \mathrm{HBE}$ cells were washed with PBS and incubated in serum-free M199 medium for 24 hours. The next day, the medium was aspirated, and cells were washed twice with PBS. Then $500 \mu \mathrm{L}$ of M199 medium with $10 \%$ FBS was added back to all wells. $16 \mathrm{HBE}$ cells were infected with $N$. meningitidis at an approximate $\mathrm{MOI}$ of 10 . Plates were covered with a Breathe-Easy sealing membrane (Sigma-Aldrich, Missouri, USA) to prevent internal contamination. Dilutions of the bacterial suspension used to infect the cells were immediately plated out on CBA plates to 
calculate the input bacteria. After 16 hours of incubation, dilutions were made of the cell culture supernatant, and were plated out on CBA. Isolates were assayed in parallel with three to five replicates per isolate. Experiments were performed three independent times.

\subsection{Cell association and invasion assays}

The number of $16 \mathrm{HBE}$ cell-associated $N$. meningitidis (adherent and intracellular) was quantified. Confluent 24-well plates of $16 \mathrm{HBE}$ cells were serum-starved for 24 hours, then washed and the medium containing $10 \%$ FBS was replaced. The cells were then infected with a fresh suspension of $N$. meningitidis for a final $\mathrm{MOI}$ of approximately 10 . Immediately after adding the bacteria to the wells, $10 \mu \mathrm{L}$ samples were taken for dilution and enumeration of CFUs. Cells and bacteria were co-incubated for one hour for cell association assays, and four hours for gentamicin protection assays. After incubation, cells were washed with warm PBS three times to remove all bacteria that were not adherent or intracellular. Cells were lysed with $500 \mu \mathrm{L}$ 1\% saponin (Sigma-Aldrich, Missouri, USA) in PBS per well, and incubated at room temperature for 10 minutes with gentle shaking. Dilutions of the cell lysate were made and plated onto CBA plates. To determine the number of intracellular bacteria, the same method was used, but prior to the addition of saponin, $500 \mu \mathrm{L}$ gentamicin $(150 \mu \mathrm{g} / \mathrm{mL})$ (Thermo Fisher Scientific, USA) was added for one hour to kill extracellular bacteria as described by Lambotin et al (2005). Gentamicin was removed and cells were thoroughly washed with PBS to remove all traces of gentamicin before addition of saponin. The cell lysates were diluted and plated onto CBA plates. Following overnight incubation, CFU were counted; input and output numbers of CFU were used to calculate percentage of intracellular or cell-associated bacteria. Isolates were assayed in parallel with three to five replicates per isolate. Experiments were performed three times. Statistical significance was tested using a one-way ANOVA.

\subsubsection{Cell-association assay with polarised $16 \mathrm{HBE}$ cells}

Transwell filters were allowed to fully polarise for three weeks as described (Section 2.2.4.3) and were checked to ensure tight junctions were intact. Before infection with $N$. meningitidis, apical and basal wells of the Transwell system were washed with PBS and replaced with serum-free 
M199 medium for 24 hours. The next day, the medium was aspirated, cells were washed twice with PBS and $600 \mu \mathrm{L}$ M199 with 10\% FBS was added to the basal well, $100 \mu \mathrm{L}$ serum-free M199 was added to the apical well. The apical well of the Transwells were infected with $N$. meningitidis to achieve an $\mathrm{MOI}$ of 10 . Ten microlitres from each apical well was removed, and serial dilutions were plated on CBA to calculate input bacteria by counting viable colony forming units. Cell and bacteria co-cultures were incubated at $37^{\circ} \mathrm{C}$ and $5 \% \mathrm{CO}_{2}$ for one hour. After incubation, basal and apical medium was aspirated, and the apical wells were washed three times with PBS to remove non-adherent bacteria. $100 \mu \mathrm{L}$ of $1 \%$ saponin was added to the apical wells and incubated at room temperature for 10 minutes with gentle agitation to lyse cells. The solution was pipetted up and down to mix, and dilutions of cell lysate were plated on CBA to determine number of adherent bacteria. CBA plates were incubated at $37^{\circ} \mathrm{C}$ with $5 \% \mathrm{CO}_{2}$ overnight and CFU were counted the following day. Isolates were assayed in parallel with three replicates. Experiments were performed three times. Statistical significance was tested using an unpaired t-test.

\subsubsection{E. coli association assay}

$16 \mathrm{HBE}$ cells were grown to confluence on a 24 well plate. E. coli expression of HpuA was either induced with $0.4 \mathrm{mM}$ Isopropyl $\beta$-D-1-thiogalactopyranoside (IPTG) or repressed with $10 \mathrm{~g} / \mathrm{L}$ glucose, and bacteria were used to infect $16 \mathrm{HBE}$ cells at an MOI of approximately 10 . The cell association assay was performed as detailed in Section 2.8, however, infection was limited to 30 minutes to reduce $E$. coli-mediated cell toxicity. Induced and non-induced $E$. coli were assayed in parallel with three replicates per condition. Experiments were performed three independent times. Statistical significance was tested using an unpaired t-test.

\subsection{Enzyme-linked immunosorbent assay}

Confluent $16 \mathrm{HBE}$ cells, in a 24 well plate, were infected with $N$. meningitidis at an $\mathrm{MOI}$ of approximately 10. Plates were covered with a Breathe-Easy sealing membrane (Sigma-Aldrich, Missouri, USA) to prevent internal contamination. Cell culture supernatants were collected 
hourly from each well for the first 12 hours, and again after 24 hours. The cell supernatants were incubated for one hour at $56^{\circ} \mathrm{C}$ to heat-kill $N$. meningitidis and were then spun at $13,000 \mathrm{x}$ $g$ for three minutes to pellet bacteria and debris. The supernatants were transferred to a fresh tube and diluted 1:4 for use in enzyme-linked immunosorbent assay (ELISA). Human Haptoglobin ELISA kit (Abcam, Cambridge, UK) and Haptoglobin Bovine ELISA Kit (Abcam, Cambridge, UK) were used according to the manufacturer's instructions to measure levels of human and bovine haptoglobin in the samples. A FLOUstar OPTIMA (BMG LABTECH) plate reader was used to measure the final OD at a wavelength of $450 \mathrm{~nm}$. The sample concentration was calculated from the standard curve and multiplying the value by the dilution factor. Isolates were assayed in parallel with three to five replicates per isolate. Significance was calculated using a one-way ANOVA.

\subsection{Anti-microbial assay}

16HBE cells were grown to confluence in a 24 well plate and infected with fresh $N$. meningitidis suspension at an estimated MOI of 10 . Bacteria were co-incubated with increasing concentrations of pooled human haptoglobin (Sigma-Aldrich, Missouri USA), to achieve a final concentration of $0 \mathrm{ng} / \mathrm{mL}$ up to $1000 \mathrm{ng} / \mathrm{mL}$. Immediately, $10 \mu \mathrm{L}$ were removed from each well and serial dilutions were plated to determine input CFU of bacteria. After 24 hours of incubation, $100 \mu \mathrm{L}$ was collected from each well and serial dilutions were plated on CBA to determine CFU of bacteria in presence of haptoglobin. Statistical significance was determined using a one-way ANOVA.

\subsection{Microscopy}

\subsubsection{Preparation of paraformaldehyde for fixation of cells}

To make a 4\% paraformaldehyde solution, $160 \mathrm{~mL}$ PBS was warmed on a hot plate with a magnetic stir bar on low until it reached approximately $60^{\circ} \mathrm{C}$. Eight grams of paraformaldehyde powder was added to the PBS. Sodium hydroxide solution (1N) was added drop-wise to slowly 
raise the $\mathrm{pH}$ until the solution cleared and the paraformaldehyde was completely dissolved. The solution was then cooled and filtered. PBS was added to adjust the final volume to $200 \mathrm{~mL}$. The final $\mathrm{pH}$ was checked and adjusted with dilute hydrochloric acid $(\mathrm{HCl})$ solution to achieve a final $\mathrm{pH}$ of 7.4. The solution was dispensed into $50 \mathrm{~mL}$ conical tubes and stored at $-20^{\circ} \mathrm{C}$.

\subsubsection{DAPI staining}

Bacterial attachment to $16 \mathrm{HBE}$ cells was detected by staining DNA (i.e., cell nuclei and bacteria) with DAPI. 16HBE cells were grown to confluence on eight-well chamber slides (Thermo Fisher Scientific, USA), and infected with fresh $N$. meningitidis at an MOI of 10 . Immediately, $10 \mu \mathrm{L}$ from each well was removed and dilutions were plated out on CBA plates to determine input number of bacteria. After infection for four hours, the medium was removed, and cells were washed three times with cold PBS. The cells were immediately fixed with $4 \%$ paraformaldehyde in PBS for 20 minutes at room temperature. The paraformaldehyde was removed by washing with PBS. Cells were permeabilised with $0.5 \%$ Triton X-100 for 10 minutes. Cells were blocked with a solution made up with 10\% heat-inactivated FBS, and 0.1\% Triton X-100 in PBS for 20 minutes, then were actin stained with Alexa Fluor 647 phalloidin (Thermo Fisher Scientific, USA) in blocking solution for 20 minutes. Cells were washed three times in PBS then stained with DAPI (4', 6-Diamidino-2-Phenylindole, Dihydrochloride) nucleic acid stain (Thermo Fisher Scientific, USA) in PBS for 10 minutes, before being washed three times with PBS. Slides were mounted with Prolong Gold Antifade Mountant (Thermo Fisher Scientific, USA) and stored in the dark at $4^{\circ} \mathrm{C}$ until analysis.

\subsubsection{Haptoglobin staining}

For immunofluorescence staining of cellular haptoglobin, 8-well chamber slides were prepared, infected, and stained as detailed for DAPI staining. After the PBS washes following DAPI staining, wells were circled with hydrophobic Mini Super HP PAP pen (Biotium, USA) and $30 \mu \mathrm{L}$ of a 1:50 dilution of primary anti-human haptoglobin polyclonal antibody (PA5-24174; Thermo Fisher Scientific, USA) was added to each well and incubated overnight at $4^{\circ} \mathrm{C}$. The following day, slides were washed three times with PBS and $4 \mu \mathrm{g} / \mathrm{mL}$ anti-rabbit Alexa Fluor 488-conjugated 
secondary antibody. One well was reserved for a control without primary antibody, and another for a control without secondary antibody. The slides were washed three times with PBS before being mounted with Prolong Gold Antifade Mountant (Thermo Fisher Scientific, USA) and stored in the dark at $4^{\circ} \mathrm{C}$ until analysis.

\subsubsection{Digital image acquisition of stained chamber slides}

Chamber slides were analysed at 100x magnification on an Olympus upright fluorescence microscope (BX-51), and images were recorded with a digital camera attached to the microscope. For each well, eight pictures were taken at random positions. Images were merged and using ImageJ (Schneider et al., 2012). 


\section{Investigation of phenotypic differences between NZ97/052 household meningococcal isolates}

\subsection{Introduction}

The processes that lead to invasive meningococcal disease (IMD) are not fully understood. However, as discussed in Chapter One, there is major evidence suggesting damage to the respiratory epithelium may increase the risk of IMD. Evidence for this includes disease rates in the African "meningitis belt", which can be some of the highest in the world. Disease rates are associated with the dry season and the Harmattan, a dust-filled wind from the Saharan desert (Agier et al., 2013; Greenwood et al., 1984; Tzeng \& Stephens, 2000). Without altering carriage rates, wind speed is correlated to rates of IMD, suggesting that risk is related to damage of the respiratory epithelium (Greenwood et al., 1984; Sultan et al., 2005; Trotter \& Greenwood, 2007). Many other environmental and host factors that damage the mucosal epithelial surface integrity have also been shown to increase the risk of IMD. This includes epithelial disruption due to smoking, or exposure to second hand smoke, which may increase the rate of meningococcal disease by $20 \%$ (Coen et al., 2006; Tzeng \& Stephens, 2000). Other causes of respiratory nasopharynx damage, such as changes in temperature or humidity, and viral or mycoplasma infections, have also been shown to increase the risk of IMD (Cartwright et al., 1991; Stephens, 1999, 2009; Stephens et al., 2007).

Colonising N. meningitidis that inhibit rapid wound repair of epithelial cells prolong their opportunity for entry into the bloodstream and increase the risk of invasive disease. Inhibition of wound repair may be one key mechanism by which virulent isolates of $N$. meningitidis breach the epithelium. Previous research at ESR revealed that disease-associated isolates inhibit wound closure effectively, while carriage isolates are much more variable, with many not inhibiting wound closure at all (Ren \& MacKichan, 2014). This suggests inhibition of cell migration may be necessary, albeit not sufficient, for the initiation of IMD. However, the mechanism for how $N$. meningitis interferes with cell migration is unknown. 
There are no animal infection models for the natural course of carriage, transmission and development of meningococcal disease, therefore molecular epidemiology of natural infection can provide clues about virulence. $N$. meningitidis is naturally competent and has highly heterogeneous genomes, therefore, comparisons of disease and carriage genomes have not yielded strictly disease-associated virulence factors (Schoen et al., 2008; Stephens, 2009). Closely related isolates, such as those recovered from household contacts, are likely to have less variants than isolates not from the same household (Ren et al., 2017). Comparing closely related isolates can provide a key tool for linking phenotypes to specific genes.

From 1991 to 2008 there were epidemic periods of meningococcal disease in New Zealand. During this epidemic, a carriage study was conducted in Auckland, where isolates of meningococcal disease were collected from the healthy household contacts of disease patients (Simmons et al., 2001). Nasopharyngeal swabs were collected from 160 disease patients and 954 household contacts of these patients. The study was carried out to estimate carriage prevalence during epidemic periods of disease and to compare meningococcal isolates collected from disease patients to those from the nasopharynx of their healthy household contacts. In 50\% of the 108 asymptomatic N. meningitidis carriers in the study, the carried strain was indistinguishable by standard laboratory typing from that of the index patient from their household (Simmons et al., 2001). Laboratory typing included multilocus sequence typing (MLST), serotyping by PCR and ELISA, and porA subtyping. A study by Ren et al showed some isolates obtained from the household contacts of the disease patients, indistinguishable by typical laboratory typing, exhibited phenotypic differences that were not easily or readily attributable to a phase variable gene (Ren et al., 2017). These phenotypic differences could reveal key bacterial factors that lead to virulence.

A trio of isolates collected from a single household during the Auckland carriage study were further investigated in this project. These isolates were identified as serogroup C ST-8 lineage, and were all identical by standard laboratory typing (Claus et al., 2003). One isolate, NZ97/052, was collected from the disease patient. The other two isolates, NZCM111 and NZCM112, were 
collected from asymptomatic carriers residing in the same household. An in vitro assay for wound repair inhibition revealed only NZ97/052 and NZCM111 were highly effective at inhibition of 16HBE human respiratory epithelial cell migration. NZCM112 appeared to have lost the ability to inhibit the cell migration of airway epithelial cells.

\subsubsection{Aims of this chapter}

Inhibiting migrating respiratory epithelial cells may be an important virulence factor for $N$. meningitidis, however the bacterial factor responsible for this inhibition is unknown. In order to learn more about the differences between the disease-associated and carriage-associated isolates the NZ97/052 household was characterised and the interaction of these isolates with host cells was investigated. Bacterial growth, inhibition of cell migration, and the capability of isolates to adhere to and invade respiratory epithelial cells were explored. The goal was to characterise interactions between $N$. meningitidis and epithelial cells in order to identify differences between isolates in the NZ97/052 household, which may lead to understanding the variability in cell migration inhibition.

\subsection{Results}

\subsubsection{Development of an epithelial cell migration assay}

The first goal of this project was to characterise the isolates in the NZ97/052 household and to confirm the differences between the isolates in their ability to prevent host cell migration. Cell re-epithelialisation is the first, and arguably most important, step in wound repair (Perrio et al., 2007; Zahm et al., 1997). The degree to which N. meningitidis inhibits cell migration was determined by adding bacteria to cells undergoing cell migration. A well-characterised in vitro scratch assay and Oris cell migration assay (Platypus Technologies) were used to measure cell re-epithelialisation (Geiser et al., 2000; Kheradmand et al., 1994). 
16HBE140- (16HBE) cells were used for these assays; this immortalised bronchial epithelial cell line forms tight junctions and effectively mimics the morphology and function of epithelia in healthy human airways (Cozens et al., 1994). The tight junctions are useful for investigating how bacteria penetrate the epithelial layer to cause IMD. To carry out scratch assays, confluent cell monolayers were scratched with pipette tip, and wound closure was assessed by microscopy. For Oris cell migration assays, 16HBE cells were grown to confluence in a collagen-coated 96well plate, fitted with rubber stoppers that prevented cells from attaching to the middle of the well. When the stoppers were removed the cells could migrate into the 'wound' area (Figure 3.1).

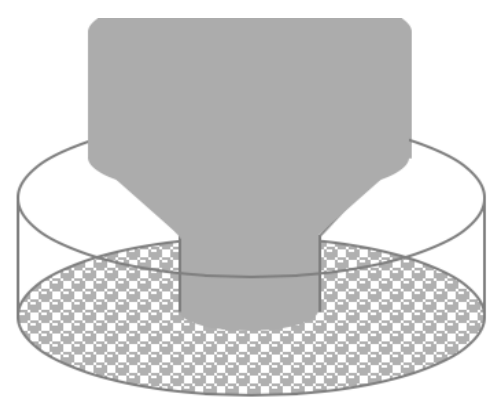

Cells seeded onto plate with stopper in place

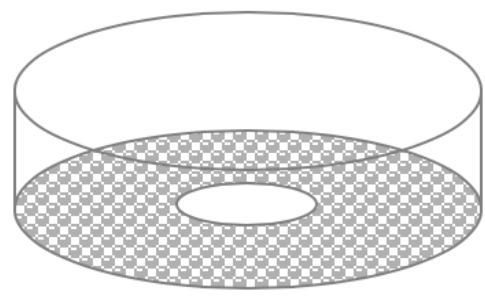

Stopper removed

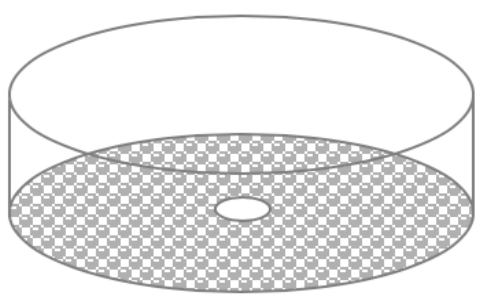

Allow cells to migrate into central region

Figure 3.1. Oris cell migration assay protocol overview. Cells were seeded into the wells of a 96-well plate, which were fitted with a stopper to prevent cells from adhering to the centre of the well. When cells reached confluence, the stoppers were removed and bacteria added. The remaining wound area was measured 16 hours later. Wells that did not have their stopper removed until the final time point were used as a proxy for the initial time point. 
Cells which had been infected with virulent and non-virulent meningococcal isolates were used. For each assay, meningococci were added after scratching the cell monolayer, or removing the rubber stopper. Following the addition of bacteria, cell cultures were placed in an incubator. After 16 hours, the wound areas were analysed by inverted microscope and were photographed to measure the remaining wound area. Within the first 24 hours, closure of this area has been shown to be due to cell migration, rather than proliferation (Kheradmand et al., 1994).

\subsubsection{Related N. meningitidis isolates differ in their ability to inhibit cell re-epithelialisation}

Previous work at ESR tested the ability of many different isolates in the meningococcal reference isolate collection to inhibit epithelial cell migration. Of all the isolates tested, one set of closely-related household isolates, identical by laboratory typing, differed in their ability to inhibit cell migration. Disease-associated isolate, NZ97/052, and carriage-associated isolates, NZCM111 and NZCM112, were tested for their ability to inhibit host cell re-epithelialisation using cell migration assays. Representative images of the Oris cell migration assay with uninfected and infected 16HBE cells are shown in Figure 3.2. 


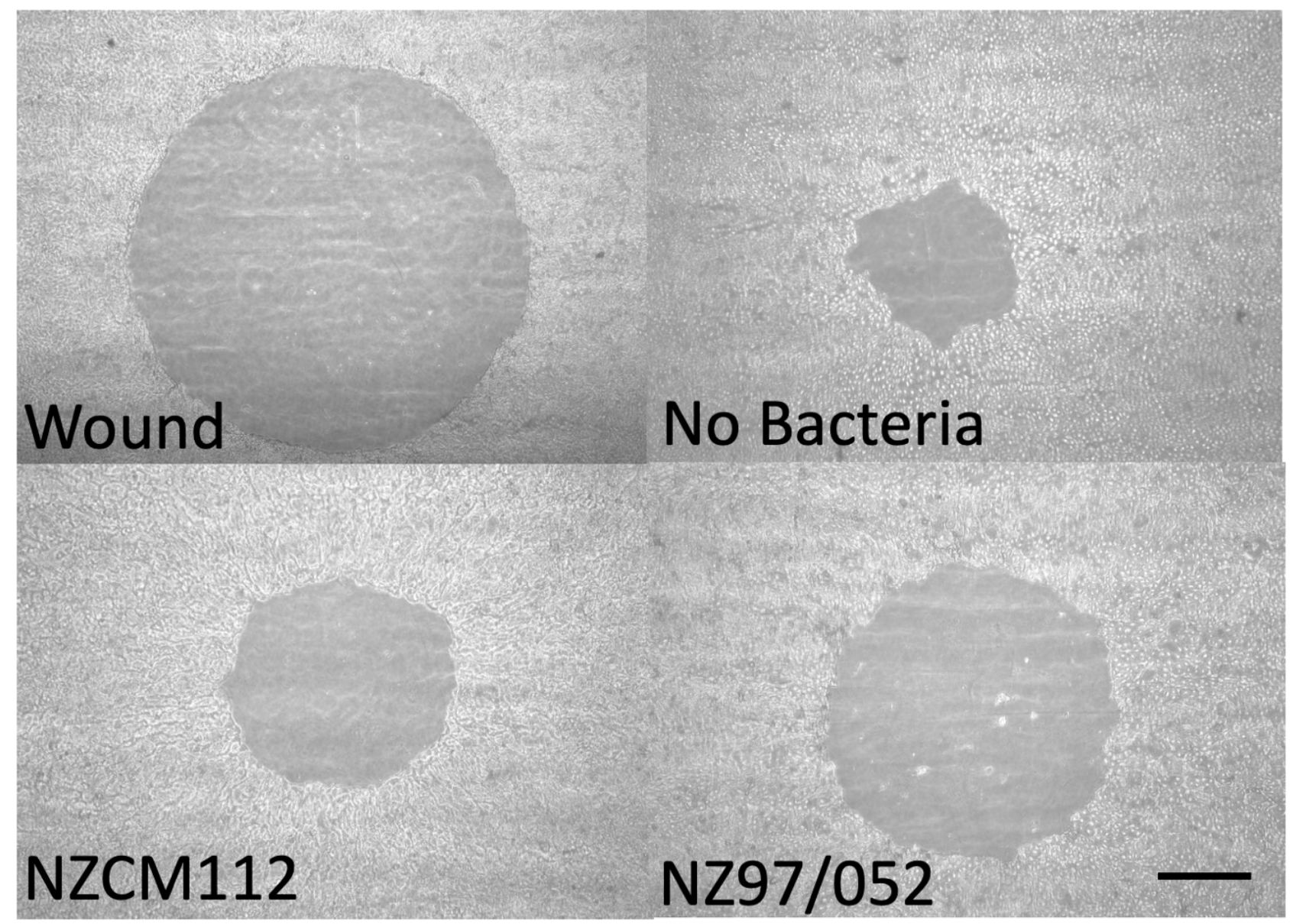

Figure 3.2. Representative photographs from Oris cell migration assay are shown, including the initial wound area (wound). Images demonstrate remaining wound area of 16HBE cells infected with NZ97/052 is much greater after 16 hours, than cells infected with NZCM112. Scale bar, $500 \mu \mathrm{m}$.

The degree of cell migration inhibition by $N$. meningitidis was determined by measuring the final wound area, and calculating it as a percentage of the wound area where stoppers were left in place as a control (i.e., initial wound area). The isolates NZ97/052, NZCM111, and NZCM112 were tested, using 12 replicates for each isolate. The results are summarised in Figure 3.3.

After 16 hours, uninfected $16 \mathrm{HBE}$ cells migrated to almost completely close the wound area, with only $12.69 \%$ of the initial wound area remaining. The nasopharyngeal carriage isolate NZCM112 was the least effective at inhibiting migrating $16 \mathrm{HBE}$ cells; the cells migrated to leave $25.15 \%$ of the initial wound area. NZCM111 and NZ97/052 isolates were much more effective at 
inhibiting cell migration, leaving $42.07 \%$ and $42.39 \%$ of the wound area respectively. The cell migration inhibition by NZ97/052 and NZCM111 did not significantly differ ( $p=0.902)$. There was a significant difference between NZ97/052 and NZCM112 isolates $(p<0.001)$ and a significant difference between NZCM111 and NZCM112 isolates $(p<0.001)$.

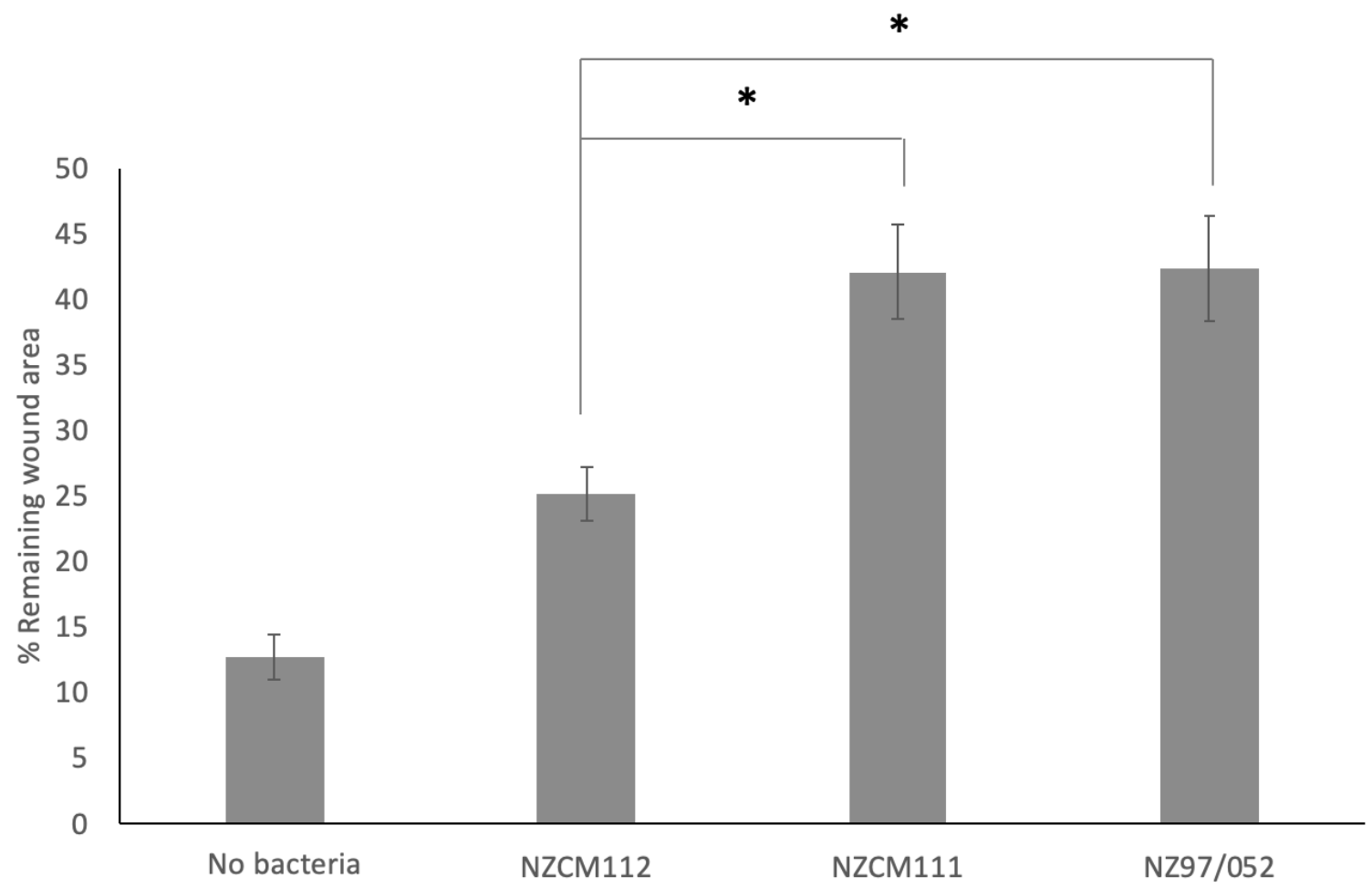

Figure 3.3. Disease isolate NZ97/052 and carriage isolate NZCM111 inhibit airway cell migration, but NZCM112 does not. Error bars show standard deviation. Data points are determined from means of 12 replicates. Significant difference from NZ97/052 was determined with One-Way ANOVA. P-value $<0.05=*$. 


\subsubsection{NZCM111 and NZ97/052 grow more efficiently than NZCM112 when cultured with 16HBE cells}

It was hypothesised that carriage-associated NZCM112 bacteria may not inhibit cell migration as they simply may not have reached as high a cell density over the 16 hours. To address this concern, $N$. meningitidis was co-cultured with $16 \mathrm{HBE}$ cells and bacterial growth assays were completed to compare growth of the isolates. Results from growth assays were compared with those of the cell migration assays to determine if any significant effects of specific isolates on cell migration inhibition could be explained due to varying numbers of bacteria.

To mimic the conditions of the in vitro wound repair assays, bacteria were grown in the presence of $16 \mathrm{HBE}$ cells and enumerated by colony forming units (CFU). Meningococcal isolates were co-cultured with bronchial epithelial cells for 16 hours. Growth was normalised to the initial inoculate, determined by CFU counts at time of infection. Bacterial growth was measured by counting CFUs for the meningococcal isolates belonging to the NZ97/052 household (Figure 3.4). Results from this assay revealed a significantly higher level $(p=0.04)$ of bacterial growth in NZ97/052 compared to NZCM112. The final number of NZCM112 cells was $38.05 \%$ that of NZ97/052. This revealed an approximately 2.5 fold difference in final cell numbers at 16 hours. The difference in final numbers of bacteria between isolates NZ97/052 and NZCM111 or NZCM111 and NZCM112 did not reach statistical significance. Growth rate could not be calculated for the CFU growth assays, as there was no way of knowing how much of the growth period the bacteria were in exponential phase, as only 0 and 16 hour measurements were taken. 


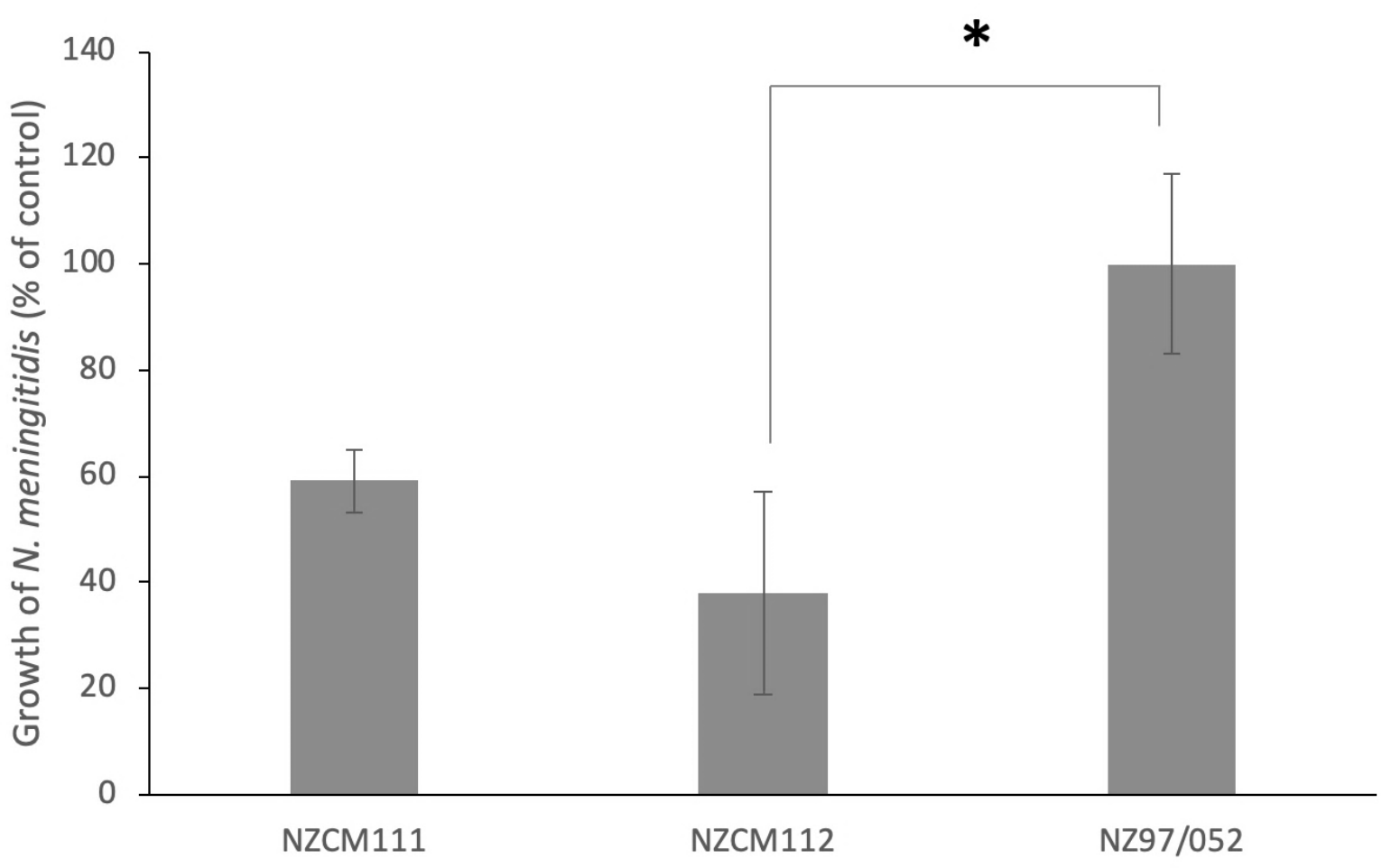

Figure 3.4. NZ97/052 grow more efficiently than NZCM112 in the presence of 16HBE cells. This experiment was performed in triplicate, three individual times. This assay compared the isolates CFU after 16 hours, relative to the starting CFU. Results are presented normalised relative to the growth of NZ97/052 isolate. ${ }^{*}$ Represents $p$ value $<$ 0.05 .

\subsubsection{Increasing the multiplicity of infection of $\boldsymbol{N}$. meningitidis does not impact host cell migration}

Results from the growth assays measuring CFU of the three bacterial isolates revealed a significant difference in the final number of viable bacteria of NZ97/052 and NZCM112 after 16 hours (Figure 3.4). To determine whether increased bacterial numbers of NZ97/052 were responsible for increased inhibition of cell migration, the cell migration assays were inoculated with higher numbers of bacteria for the NZCM112 isolate. The focus of these experiments was to investigate the difference between disease- and carriage-associated isolates which differed in their ability to inhibit cell migration. Therefore, NZCM111 was excluded from this assay. 
The estimated multiplicity of infection (MOI) that was typically used for all cell migration assays was 10, meaning approximately 10 meningococcal cells were inoculated for each $16 \mathrm{HBE}$ cell. Here the Oris cell migration assays were completed with a range of bacterial MOI, including 10, 15, and 20 (Figure 3.5). After 16 hours, 16HBE cells infected with NZCM112 at an MOI of 10, 15, and 20 had wound areas which measured $22.48 \%, 22.65 \%$, and $24.62 \%$ of the initial wound area respectively $(n=8)$, which did not significantly differ. Cells infected with NZ97/052 at MOI of 10, 15 , and 20 also did not differ significantly in their levels of cell migration inhibition. Wound areas at 16 hours were $42.28 \%, 42.34 \%$, and $44.97 \%$ respectively. Infection of $16 \mathrm{HBE}$ cells with higher levels of bacteria did not lead to increased inhibition of cell migration by either NZ97/052 or NZCM112. This result suggests that inhibition of cell migration was independent of the differing concentrations of bacteria tested. Therefore, it is unlikely the difference in inhibition of cell migration between NZ97/052 and NZCM112 can be explained by the difference in bacterial growth.

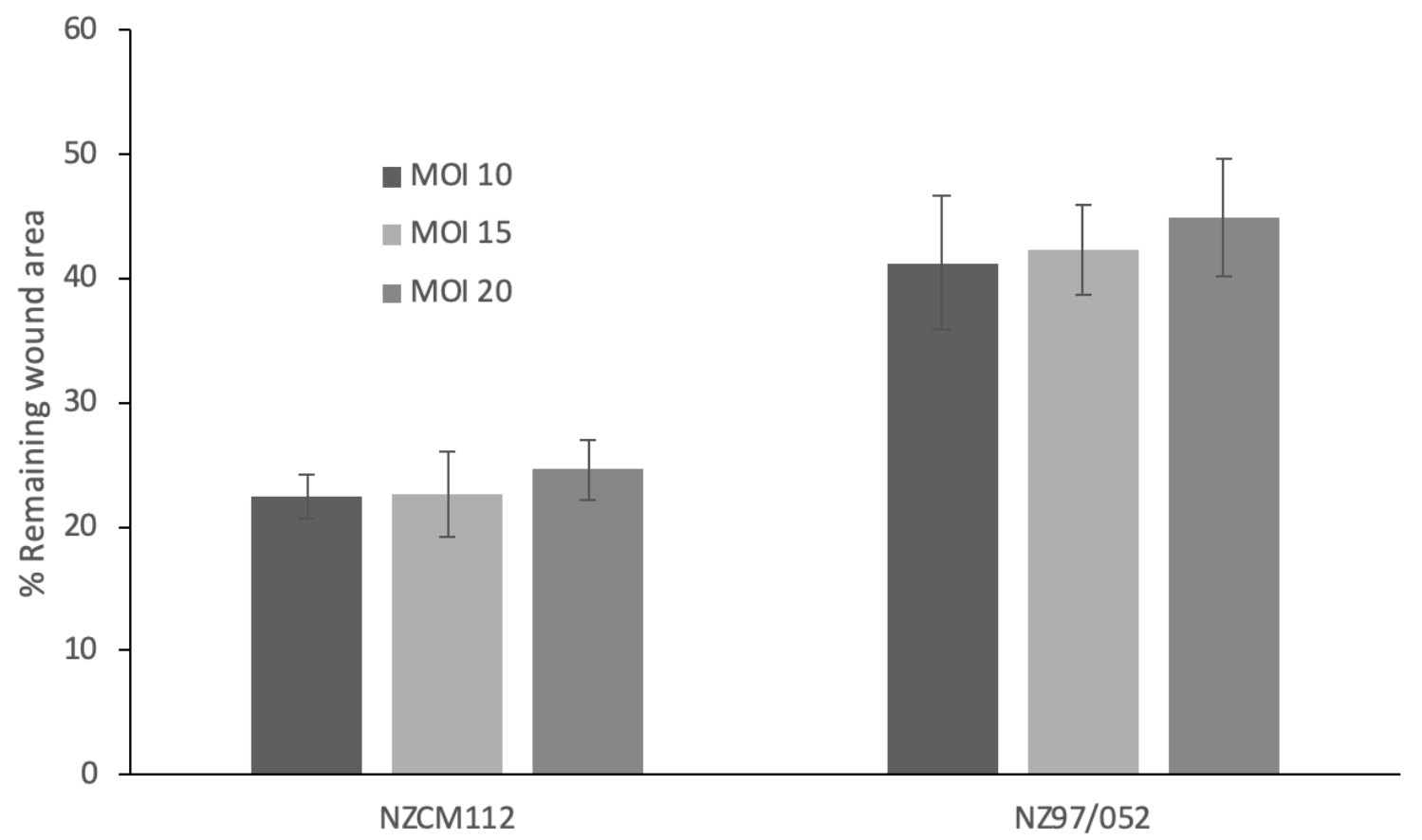

Figure 3.5. The inhibition of cell migration did not vary significantly with increasing multiplicity of infection of meningococci. Error bars show standard deviation. Data points were determined from means of eight replicates. 


\subsubsection{NZ97/052 has greater association to $16 \mathrm{HBE}$ cells than NZCM112}

Adherence to respiratory epithelial cells is essential for meningococcal colonisation. The ability for bacteria to be taken up by non-phagocytic cells, such as respiratory epithelial cells, may provide a sheltered niche and opportunity to evade the human immune system, and has been suggested to contribute to the development of IMD (Hill et al., 2010; Stephens et al., 1983). It is unknown if bacterial adherence or invasion can cause an interruption of cell migration, therefore, association assays and gentamicin protection assays were completed to determine if NZ97/052 and NZCM112 differ in their adhesion or invasion of human host cells.

Adherence was determined by measuring viable $N$. meningitidis closely associated with respiratory epithelial cells. The association assays cannot exclude intracellular bacteria, however cellular invasion occurs at a much lower rate than adhesion. Cell association is therefore a good representation of adherence. Mid-logarithmic phase meningococci were co-cultured with $16 \mathrm{HBE}$ cells. After an incubation period of one hour, cells were carefully and thoroughly washed to remove non-adherent bacteria. Saponin was used to lyse $16 \mathrm{HBE}$ cells, without affecting meningococcal viability. Enumeration of cell-associated bacteria was determined by dilution plating and counting colonies the following day. Cell association of NZCM112 and NZ97/052 was calculated as a percentage of the initial inoculant for each, also enumerated by CFU (Figure 3.6A). The NZ97/052 isolate displayed significantly higher levels of association with 16HBE cells; about 1.8 fold higher than NZCM112 ( $p<0.001)$. These results suggest that cell association and the inhibition of cell migration are linked. This is possibly because adherence is necessary for inhibition of cell migration, or perhaps bacteria must adhere to deliver a virulence factor that interrupts cell migration.

A gentamicin protection assay was used to enumerate intracellular bacteria in $16 \mathrm{HBE}$ cells. Gentamicin was used to kill all of the extracellular bacteria after co-culturing $N$. meningitidis and $16 \mathrm{HBE}$ cells for four hours. A longer incubation period was used than that for the association assays, as invasion is a more complex process than adhesion and typically takes longer. Gentamicin was removed, and the cells were washed and lysed with saponin detergent to 
release the intracellular bacteria for plating and enumeration. The original inocula of both NZCM112 and NZ97/052 added to the cell cultures were also counted to calculate the intracellular bacteria as a percentage of the initial inoculum. Intracellular CFU of the NZ97/052 isolate, relative to the input, were significantly more abundant than those of intracellular NZCM112 CFU ( $p=0.011$ ) (Figure 3.6B). The difference in invasion between NZ97/052 and NZCM112 follows the same trend as levels of cell association. Intracellular bacteria account for a very small fraction of all cell-associated bacteria, which suggests the higher level of invasion seen in NZ97/052 results from higher numbers of bacteria adhering to host cells. 


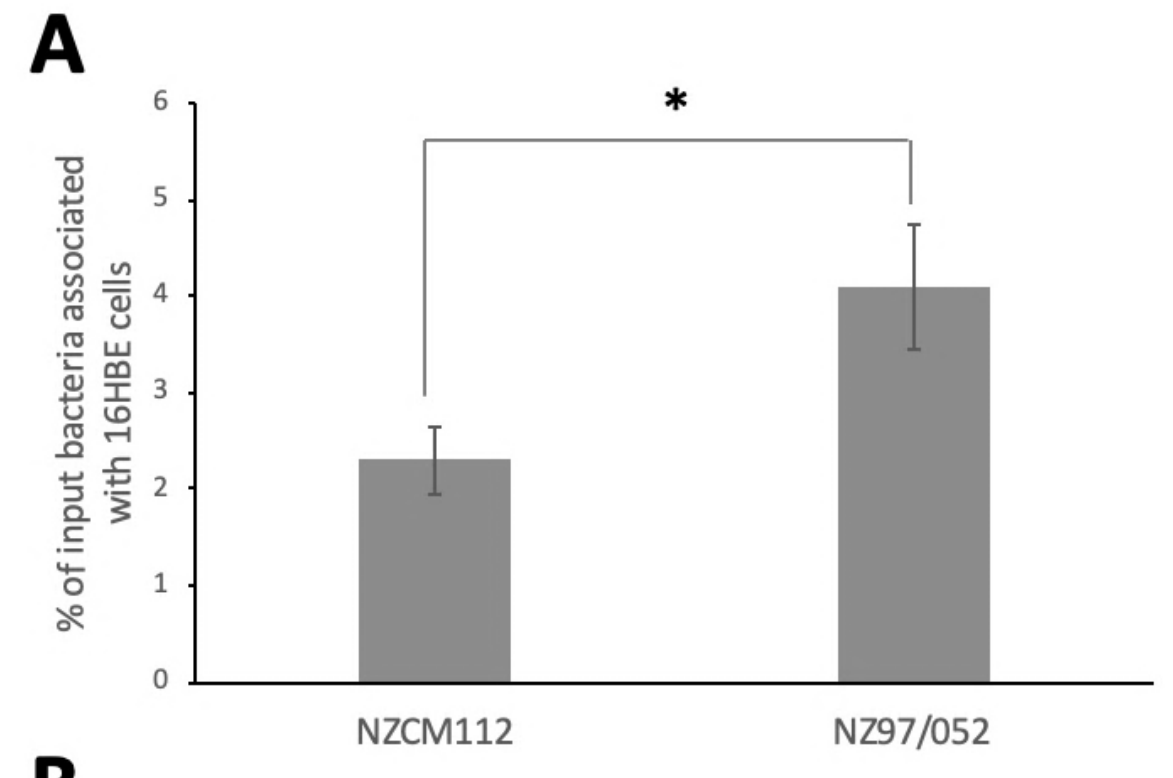

B

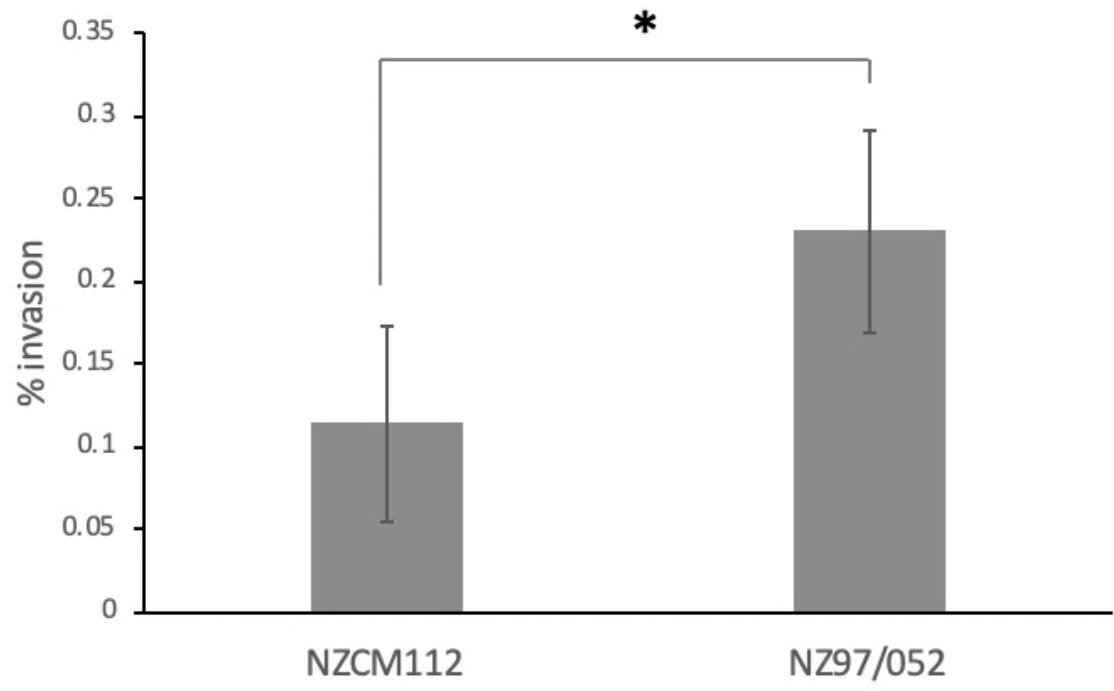

Figure 3.6. NZ97/052 has higher levels of association to 16HBE cells compared to NZCM112. (A) NZ97/052 had higher rate of cell association, as a percent of input bacteria, relative to NZCM112, after one hour. (B) NZ97/052 had higher rate of cell invasion, as a percent of input bacteria, relative to NZCM112, after four hours. Both experiments were performed in triplicate, three independent times. Error bars represent standard deviation. * Represents $p$-value $<0.05$. 


\subsubsection{NZ97/052 isolate has higher association to polarised human respiratory epithelial cells compared to NZCM112}

An association assay in a Transwell system was used to confirm the results from the association assays (Figure 3.6), using a cell culture model that more closely represents the respiratory epithelium in the human host. A Transwell filter system can be used to develop polarised layers of epithelial cells, to mimic the configuration of intact tissue polarity. This system allows tight separation of the apical and basolateral regions of the epithelium (Figure 3.7). 16HBE cells, when grown on a Transwell filter, polarise and form a barrier with tight junctions between the two chambers preventing medium in the basal chamber from leaking into the apical chamber (Pentecost et al., 2006; Vogelmann \& Nelson, 2005). 16HBE cells were grown in a monolayer on a permeable membrane, and were fed basolaterally with a nutrient-rich medium. The basolateral medium was M199 supplemented with 10\% foetal bovine serum, while the apical medium, above the $16 \mathrm{HBE}$ cells, was serum-free M199 medium.

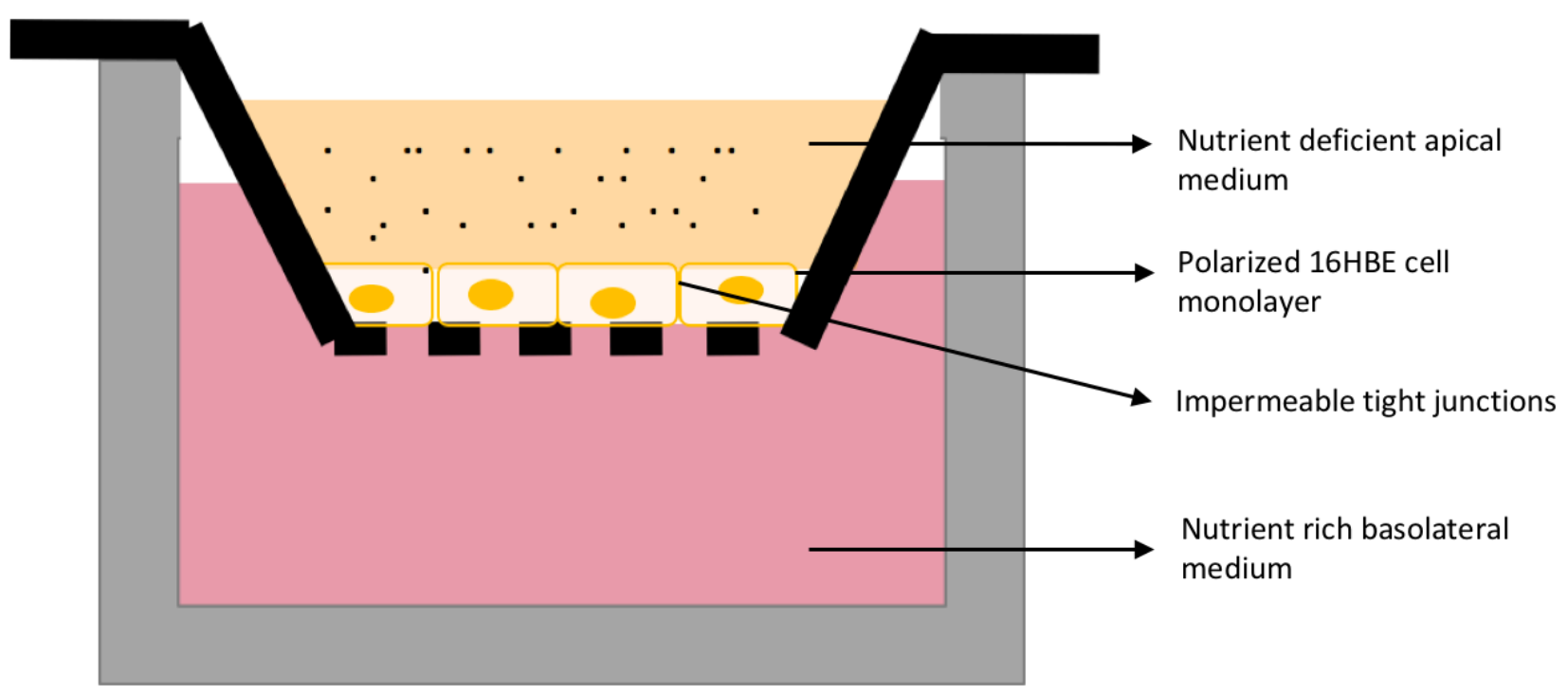

Figure 3.7. Transwell system showing basal and apical chambers 
16HBE cells were grown to a monolayer on the permeable membrane of the Transwell chamber and allowed to fully polarise for three weeks. The Transwell cell monolayers were tested for leaks using Evans blue, a molecule which that binds to serum albumin (Hawkins \& Egleton, 2006). Transport of Evans blue is considered to represent albumin transport, which does not occur across an intact cellular barrier (Hawkins \& Egleton, 2006). Leakage between apical and basolateral wells was detected by spectrophotometer. Cell monolayers were determined to be intact as there was no significant leakage between wells (Appendix, Figure 9.1). Apical wells were infected with $N$. meningitidis and cell association assays were completed as described in Section 2.8.1. Results from the association assays using the Transwell system (Figure 3.8) were consistent with the results from the 16HBE monolayer association assays (Figure 3.6). The NZ97/052 isolates displayed higher levels of association to $16 \mathrm{HBE}$ cells (3.5\% of input) relative to NZCM112 (1.4\% of input). This was an approximately 2.5 fold increase in association, and the difference was statistically significant $(p=0.032)$. This result suggests that using non-polarised cells is a sufficient model, and there does not appear to be any specific features of the apical surface which alter bacterial adherence.

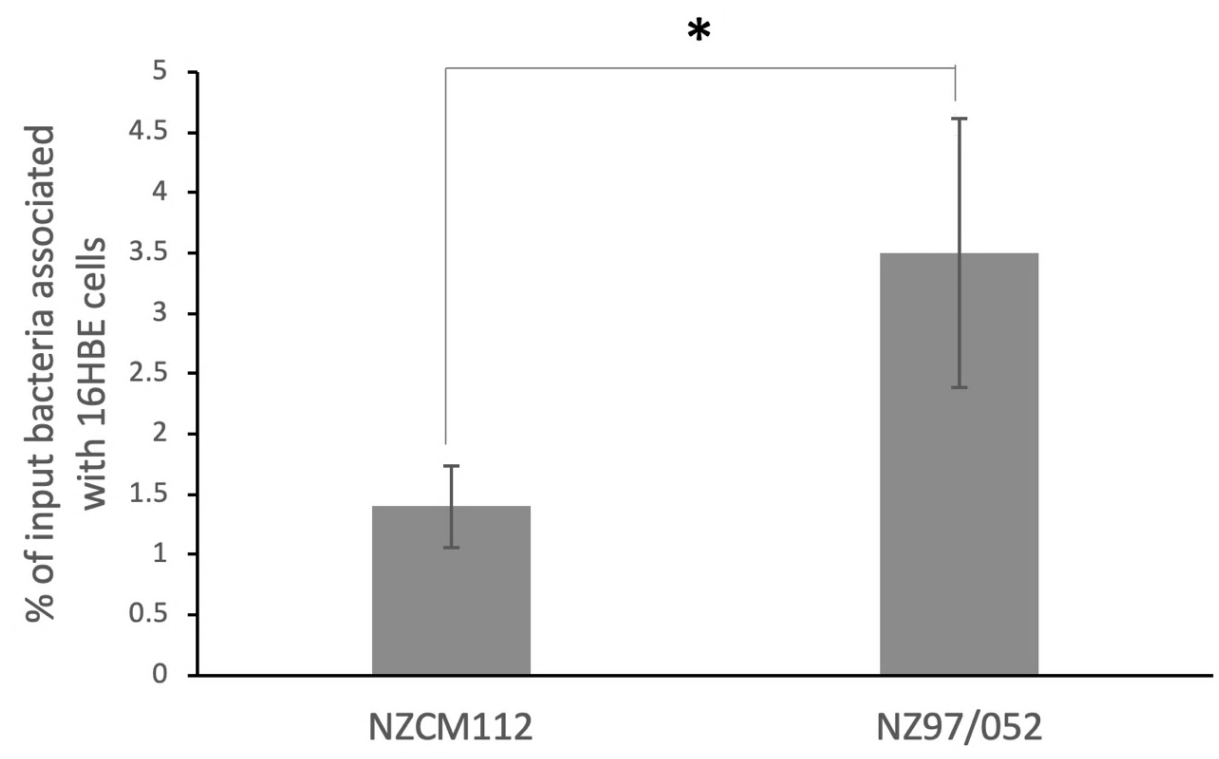

Figure 3.8. NZ97/052 had significantly higher association with polarised 16HBE cells, relative to NZCM112, on the apical surface in a Transwell system. Experiments were performed in triplicate, two independent times. Error bars represent standard deviation. ${ }^{*}$ Represents $p$-value $<0.05$. 


\subsection{Discussion}

Due to the lack of animal models for $N$. meningitidis nasopharyngeal carriage and the natural route of infection, there is a major gap in the understanding of how the bacteria residing in the nasopharynx can cause invasive disease. Disease-associated isolates of $N$. meningitidis are effective at inhibiting respiratory epithelial cell migration, while carriage-associated isolates are variable (Ren \& Mackichan, 2014). The inhibition of cell migration is potentially key to understanding how bacteria, which normally reside in the nasopharynx, can occasionally breach the epithelium barrier causing invasive disease. Inhibiting wound closure may provide bacteria a prolonged opportunity to invade deeper tissues and cause IMD.

Previous attempts at ESR to identify the meningococcal factor responsible for bacterial inhibition of cell migration have not been successful (Ren \& MacKichan, 2014). However, in this study, a trio of closely-related isolates collected from a single household were identified. These isolates, NZ97/052, NZCM111, and NZCM112, were identical by standard laboratory typing. However, carriage-associated isolate NZCM112 showed a significantly decreased ability to prevent epithelial cell migration in an in vitro model, relative to the related clinical isolate, NZ97/052. These isolates may be useful in understanding the process of inhibition of cell migration. The goal of this chapter was to characterise the interactions of these closely related isolates with $16 \mathrm{HBE}$ cells, and to identify additional differences between the isolates which may contribute to virulence and wound repair inhibition.

Growth of these meningococcal isolates was investigated to detect any differences between NZ97/052 and NZCM112. This was completed to determine whether the differences in cell migration inhibition could be explained by variable numbers of viable bacteria. The diseaseassociated isolate, NZ97/052, grew to higher final numbers of viable cells compared to the carriage associated isolate NZCM112. In order to determine whether this difference in growth is responsible for altered cell migration inhibition, Oris cell migration assays were completed with increasing concentrations of input bacteria. Higher numbers of infecting bacteria did not 
significantly change cell migration inhibition, for either NZ97/052 or NZCM112. It is therefore unlikely that the difference in cell migration inhibition between isolates is solely due to the more rapid growth of NZ97/052.

Tight adherence of meningococci to airway epithelial cells is thought to be important in carriage, although the significance of cellular invasion remains unclear. The importance of meningococcal adherence and invasion of epithelial cells in the inhibition of wound repair is also unknown. Cell association and invasion assays demonstrate the ability of bacteria to adhere to and invade normally non-phagocytic host cells.

There are significant gaps in what is currently known about N. meningitidis invasion of epithelial cells. Many harmless carriage isolates can often invade host cells, therefore entry into epithelial cells may not be the route of invasive disease (Stephens, 2009; Stephens et al., 1983). Adherence to respiratory epithelial cells is clearly necessary but not sufficient for causing invasive disease. Adherence is equally important for carriage isolates, carried asymptomatically, and also for other non-virulent Neisseria species, including N. lactamica and N. polysaccharea (Hung \& Christodoulides, 2013). However, association with 16HBE cells was significantly higher for NZ97/052 than for NZCM112. These results mirrored those seen in the cell migration assays. This may suggest a possible link between cell association and the inhibition of cell migration, potentially due to interactions between the bacteria and respiratory epithelial cells. The bacteria may adhere to host cells to deliver a virulence factor that prevents cell migration or, alternatively, migration of host cells may be interrupted by alteration of cell signalling.

The gentamicin protection assay revealed NZ97/052 had higher invasion of 16HBE cells than NZCM112. This result is likely explained by increased adherence of the bacteria to the epithelial cells, but it is possible that intracellular bacteria are important for the inhibition of cell migration. Perhaps once inside the cells, $N$. meningitidis interferes with normal cellular signalling or actin dynamics necessary for cell migration. This hypothesis has yet to be tested. 
N. meningitidis virulence is believed to be polygenic (Joseph et al., 2010), and development of invasive disease is likely the result of the contribution of many genes. Therefore, studying large numbers of meningococcal genomes may not yield genes strictly involved in virulence, however these experiments show the utility of using closely related household genes which differ phenotypically. The major meningococcal virulence factors are those which enable the bacteria to multiply rapidly in the bloodstream. Our goal was to understand what other factors may be important in the nasopharynx, and how they may contribute to entry of the bacterium into the bloodstream.

\subsection{Summary}

Various differences in closely related disease- and carriage-associated meningococcal isolates were revealed. Experiments completed in this chapter confirmed the difference in the ability to inhibit cell migration between disease-associated and carriage-associated isolates of the NZ97/052 household. Bacterial growth in a cell co-culture was also significantly higher for NZ97/052 than NZCM112, however increasing the starting numbers of bacteria did not impact the cell migration assay, suggesting the difference in growth does not directly impact inhibition of cell migration. Association assays also identified NZ97/052 had higher levels of association with $16 \mathrm{HBE}$ cells than NZCM112, indicating a possibility that adherence to $16 \mathrm{HBE}$ cells and inhibition of cell migration may be linked. These differences are remarkable in light of how similar these isolates are. 


\section{Investigation of $\boldsymbol{N}$. meningitidis metabolism and host cell migration}

\subsection{Introduction}

'Nutritional immunity' is the process by which the host limits the growth of invading pathogens by restricting access to essential nutrient metals (Hood \& Skaar, 2012; Stork et al., 2013). In order for successful bacterial colonisers to survive and replicate inside a host, they have developed strategies to overcome the limitation of host resources, and scavenge essential nutrients (Perkins-Balding et al., 2004). Metabolism has therefore shown to play an important role in bacterial virulence, as it allows bacterial pathogens to exploit host resources (Schoen et al., 2014; Shelburne et al., 2008). Essential metals, such as iron and zinc, are particularly scarce on mucosal surfaces, such as the nasopharyngeal airways. Bacteria that normally reside in this environment have evolved multiple mechanisms for scavenging these nutrients.

The inhibition of respiratory epithelial cell migration by N. meningitidis was previously shown to be independent of many surface adhesins and other bacterial factors, such as pili, that are known to interact with the host cell (Ren \& MacKichan, 2014). However, preliminary transcriptome comparisons between disease isolates, that inhibit wound repair, and unrelated carriage isolates, that do not, suggested a role for bacterial metabolism in the inhibition of epithelial cell migration (Ren \& MacKichan, unpublished data).

It is becoming clear there are key metabolic differences between hypervirulent and carriageassociated meningococcal isolates; isolates often vary in their metabolism, for instance having differing rates of nutrient consumption. (Schoen et al., 2014). Nutrient deficiency in the host is known to impair wound repair and increase vulnerability to infectious diseases (Katona \& Katona-Apte, 2008; Thompson \& Fuhrman, 2005). It is possible consumption of nutrients by certain meningococcal isolates could result in "starving" the host cells of factors required for epithelial cell migration. 


\subsubsection{Transition metals, amino acids, and nutritional immunity}

Nutrients, particularly transition metals, are crucial in many biological processes.

Meningococcal transport systems for certain metals, such as iron, have been well established

(Perkins-Balding et al., 2004). However others, such as those which acquire zinc, are currently less well understood.

Iron is essential for almost all bacteria, apart from a few examples, e.g. Borrelia burgdorferi and Lactobacillus spp., that do not use iron at all (Pandey et al., 1994; Posey \& Gherardini, 2000). N. meningitidis requires iron, and it is known to contribute to its pathogenesis (Perkins-Balding et al., 2004). Although iron is the most copious transition metal in the human host, free ferrous iron $\left(\mathrm{Fe}^{2+}\right)$ is extremely scarce, due to mechanisms for withholding iron from bacteria and preventing infection (Porcheron et al., 2013). Free iron levels vary throughout the body but the mean value is $10^{-18} \mathrm{M}$, which is much lower than the level required for optimal bacterial growth, $10^{-6} \mathrm{M}$ (Rohmer et al., 2011). Meningococcal systems for iron acquisition are detailed in Chapter One. Meningococci can obtain iron from human transferrin, lactoferrin, haem, haemoglobin, and haptoglobin-haemoglobin complexes (Perkins-Balding et al., 2004; Weinberg, 2009). Some research suggests $N$. meningitidis may also be able to utilise siderophores secreted by other bacteria (Rutz et al., 1991).

Zinc is an essential structural or catalytic co-factor for many enzymes and DNA-binding proteins (Grass et al., 2002; Ohalloran, 1993). Zinc is required by N. meningitidis and consequently, bacteria have evolved mechanisms to acquire adequate intracellular zinc concentrations from host sources. Zinc is second only to iron in abundance of trace metals in the human body, although free zinc levels vary considerably in different tissues. The average levels of zinc are $120 \mu \mathrm{M}$ in the blood, $18 \mu \mathrm{M}$ in the serum, and less than $76 \mu \mathrm{M}$ in the urine (Kaur et al., 2014). In zinc-deficient environments, the human protein ZIP-4 (Guerinot, 2000) is rapidly expressed on the microvilli of enterocytes to compensate for inadequate zinc intake (Lansdown et al., 2007). In Gram-negative bacteria, ZupT, a low affinity zinc transporter also belonging to the ZIP protein family, is thought to be responsible for acquisition of zinc in limiting conditions (Cerasi 
et al., 2013). However, previous work done in our laboratory at ESR demonstrated that deletion of this gene in disease-associated meningococci did not affect the ability of the bacteria to inhibit host cell wound repair (Greig, 2014). Neisseria meningitidis, and some other Gramnegative bacteria, encode ZnuD, a TonB-dependent receptor also involved in zinc uptake (Stork et al., 2010).

Zinc oxide is widely used to promote wound repair, both as an oral supplement and as a topical application (Agren, 1990). Zinc is reported to play an important role in promoting reepithelialisation; a study by Lansdown et al demonstrated that many biochemical and molecular events in wound repair are accelerated by the addition of supplementary zinc (2007). Zinc ions mimic the action of growth factors by enhancing intracellular mitogenic signalling pathways and there is suggestion that zinc involvement in DNA polymerases causes the burst of mitosis that precedes epithelialisation (Lansdown et al., 2007). However, other research suggests that zinc supplementation increases rates of wound repair only in zinc deficient conditions (Thompson \& Fuhrman, 2005).

Essential amino acids are those which humans must obtain from their diet, while non-essential amino acids may be synthesised, mainly from glucose. Many pathogens scavenge amino acids from their hosts and have evolved ways to evade nutritional immunity that hosts uses to starve bacteria of nutrients (Zhang \& Rubin, 2013). The meningococcal genome encodes all pathways for the biosynthesis of amino acids, but also encodes several transporters for amino acids (Lipsitch \& Moxon, 1997). Non-essential amino acids, such as glutamine and arginine, are thought to be involved in wound repair, and there is suggestion that supplementation with these amino acids may increase wound repair rates (Collins, 2002; Ellinger, 2014; Kesici et al., 2015). 


\subsubsection{Aims of this chapter}

Current literature suggests nutrients, such as iron, zinc, and non-essential amino acids, may play a role in cell re-epithelialisation (Collins, 2002; Ellinger, 2014; Kesici et al., 2015; Lansdown et al., 2007). This led to the hypothesis that the inhibition of epithelial cell wound repair by $N$. meningitidis could be due to the bacterial metabolism and depletion of nutrients in the medium necessary for host cells to migrate. The trio of NZ97/052 household isolates, which differ in their ability to inhibit cell migration, were used in this chapter to investigate whether bacterial metabolism is involved in host cell wound repair. If inhibition of cell migration by diseaseassociated isolates of $N$. meningitidis resulted from their efficient uptake of essential nutrients, supplementing the bacteria and host cell co-cultures could compensate for NZ97/052-mediated inhibition of epithelial cell migration. The impact of nutritional supplementation was assessed for cell migration assays, growth rate analysis, cell invasion, and cell association assays. Diseaseand carriage-associated isolates were supplemented with nutrients important for meningococcal growth and cell wound repair.

\subsection{Results}

\subsubsection{Supplementation with non-essential amino acids or zinc does not affect wound repair inhibition}

It was hypothesised that bacterial consumption of key nutrients could be directly resulting in impaired cell migration in the wound repair assay. To test this, cell migration inhibition by disease- and carriage-associated $N$. meningitidis was measured in the presence of additional nutrients. Co-cultures were supplemented with nutrients that have been shown to be essential for bacterial growth, cell migration, or are limited by host cells in nutritional immunity (Lansdown et al., 2007; Weinberg, 2009; Zhang \& Rubin, 2013). Evidence suggests that nonessential amino acids (NEAA) and zinc are both important in wound repair (Collins, 2002; Lansdown et al., 2007). If $N$. meningitidis are consuming these important nutrients, adding these nutrients back to damaged epithelial layers may result in restoration of normal wound 
healing. To test this theory, epithelial cell wound repair assays were completed for $16 \mathrm{HBE}$ cells infected with NZ97/052 and NZCM112 with and without amino acid and zinc supplementation.

Supplementation with $2 \%$ NEAA had no significant effect on the inhibition of cell migration of $16 \mathrm{HBE}$ cells that were uninfected ( $p=0.901)$, or infected with NZCM112 ( $p=0.872)$ or $\mathrm{NZ97/052}(p=0.760)$ (Figure 4.1A). Addition of $20 \mu \mathrm{M}$ zinc sulfate also did not have a significant impact on migration of 16HBE cells infected with NZCM112 $(p=0.213)$ or NZ97/052 ( $p=0.191)$ (Figure 4.1B). Zinc supplementation had the largest effect on cell migration of uninfected 16HBE cells; at 16 hours the wound area was $12.7 \%$ of the initial wound area without $\mathrm{ZnSO}_{4}$ supplementation, and $8.88 \%$ of the initial wound area with $20 \mu \mathrm{M} \mathrm{ZnSO} \mathrm{Z}_{4}$ supplementation. However, this was a modest change in final wound area and it did not reach significance $(p=$ 0.117). Supplementation of cell cultures with NEAA or zinc did not affect cell migration or the inhibition of this process by $N$. meningitidis. These results suggest that neither NEAA nor zinc are limited under these culture conditions, and that the inhibition of wound repair does not result from their limitation. 

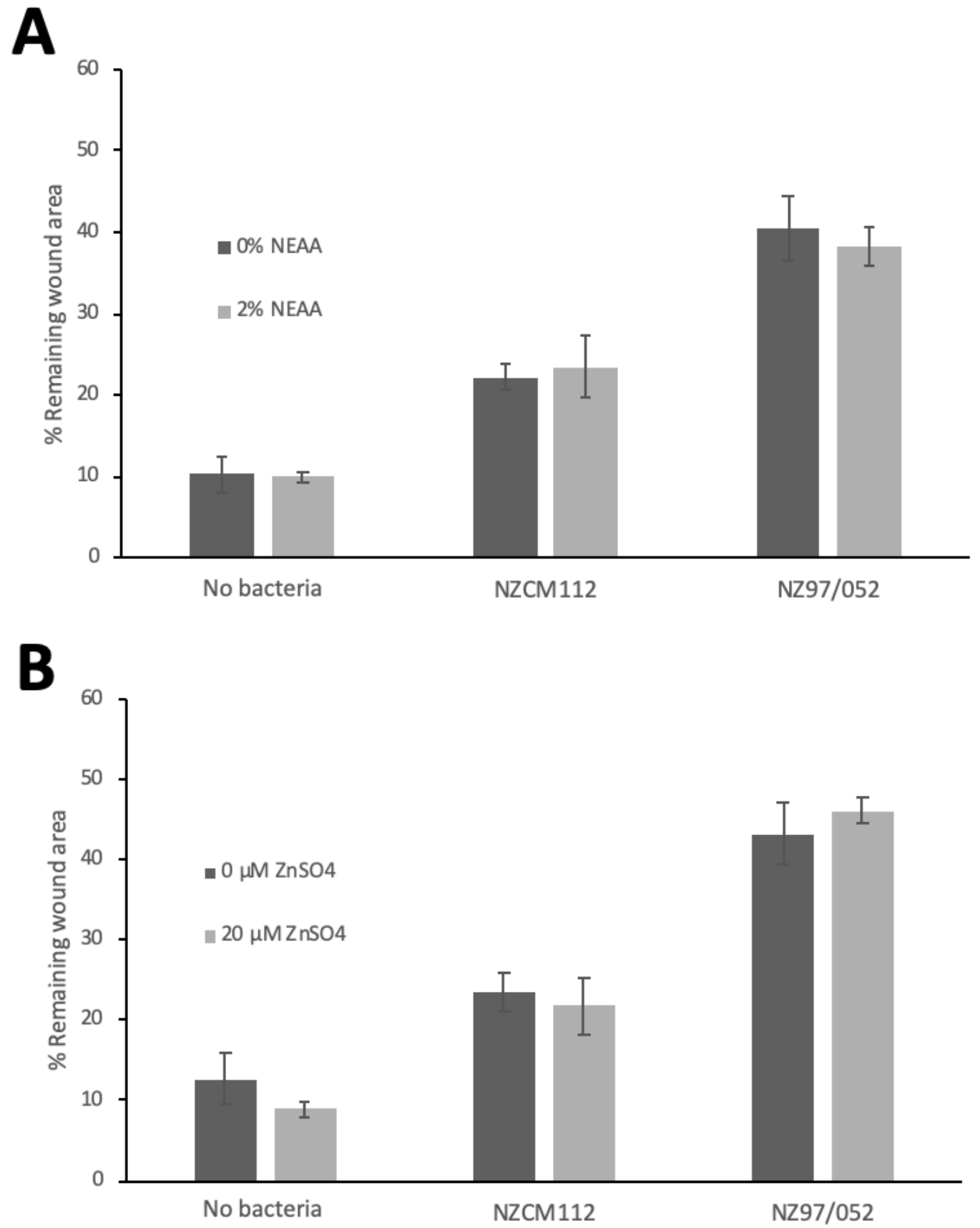

Figure 4.1. Supplementation of the cell migration assay with zinc and non-essential amino acids (NEAA) did not affect cell migration, either with or without meningococci. Addition of $2 \% \mathrm{NEAA}(\mathrm{A})$ or $20 \mu \mathrm{M} \mathrm{ZnSO} \mathrm{Z}_{4}$ (B) did not have a significant effect on wound repair inhibition. Error bars show standard deviation. Data points are determined from means of eight replicates. 


\subsubsection{Iron supplementation increases wound repair inhibition by carriage-associated isolate NZCM112}

Iron is essential to virtually all cells, including $N$. meningitidis. Limiting bacterial access to iron is an important host defence, and the majority of bacterial pathogens have therefore evolved to compete for iron (Weinberg, 1975, 2009; Wooldridge \& Williams, 1993). It was hypothesised that $N$. meningitidis may consume iron, which is essential for respiratory epithelial cells, thus limiting its availability to host cells and impacting migration.

Epithelial cell wound repair assays were completed with iron supplementation to determine whether adding iron to the cells could overcome the inhibition of cell migration by $N$. meningitidis. Iron sulfate $\left(\mathrm{Fe}^{2+}\right)$ and iron chloride $\left(\mathrm{Fe}^{3+}\right)$ were both used as iron sources to determine whether different iron oxidation states could have different effects on the inhibition of cell migration. Oris cell migration assays were supplemented with iron chloride (Figure 4.2A). Twenty micromoles of iron chloride $\left(\mathrm{FeCl}_{3}\right)$ significantly improved cell migration of uninfected $16 \mathrm{HBE}$ cells $(p=0.032)$. After 16 hours the wound area was $12.64 \%$ of the initial area without $\mathrm{FeCl}_{3}$ supplementation, and $6.32 \%$ of the initial wound area with $20 \mu \mathrm{M} \mathrm{FeCl}_{3}$ supplementation. The NZ97/052 isolate was able to inhibit cell migration to a slightly greater degree with $\mathrm{FeCl}_{3}$ supplementation (47.94\% initial wound area vs $43.32 \%$ initial wound area without $\mathrm{FeCl}_{3}$ ), but this effect was not significant $(p=0.064)$. However, surprisingly, there was a significant increase in the ability of the NZCM112 isolate to inhibit cell migration, with the final wound area increasing from $23.62 \%$ without iron, to $43.47 \%$ with iron $(p<0.001)$. This increase was so large that the ability of NZCM112 to inhibit cell migration was no longer significantly different from that of the disease-associated isolate, NZ97/052 ( $p=0.942)$. The results with iron sulfate were very similar, although it did not have a significant effect on uninfected migrating 16HBE cells $(p=$ 0.068) (Figure 4.2B) or on 16HBE cells infected with the disease-associated isolate, NZ97/052 ( $p$ $=0.081$ ). However, as seen with $\mathrm{FeCl}_{3}$, addition of iron sulfate resulted in a large and significant increase in the ability of the carriage-associated isolate, NZCM112, to inhibit cell migration. The addition of $20 \mu \mathrm{M} \mathrm{FeSO}_{4}$ resulted in a final wound area that was $43.47 \%$ of the initial wound area, compared to $23.62 \%$ of initial wound area without iron supplementation $(p<0.001)$. 
Inhibition of cell migration by NZCM112, with $\mathrm{FeSO}_{4}$ supplementation, no longer significantly differed from that observed with the NZ97/052 isolate $(p=0.922)$.

It was investigated if increasing concentrations of $\mathrm{FeSO}_{4}$ would have a dose-dependent effect on the inhibition of cell migration, or if a minimum threshold concentration that had an effect on wound repair could be identified (Figure 4.2C). This effect did not appear to be dosedependent; instead an increase in cell migration inhibition by NZCM112 at concentrations of 1 $\mu \mathrm{M}$ or higher was observed, with no effect seen at a concentration of $0.1 \mu \mathrm{M} \mathrm{FeSO}_{4}$.

The results from these experiments were unexpected, as it had been hypothesised that supplementation with iron would decrease the ability of disease-associated isolate to inhibit respiratory epithelial cell wound repair by replacing nutrients essential for $16 \mathrm{HBE}$ cells that were consumed by the bacteria. It had not been predicted that iron supplementation would have an impact on the ability of NZCM112 to inhibit wound repair. These results suggested that the initial hypothesis was not correct. Instead, it was found that iron conferred the ability of one carriage-associated isolate NZCM112 to inhibit wound repair, to similar levels as those seen with $\mathrm{NZ97/052}$. Furthermore, this effect was seen with both $\mathrm{FeCl}_{3}\left(\mathrm{Fe}^{3+}\right)$ and $\mathrm{FeSO}_{4}\left(\mathrm{Fe}^{2+}\right)$ supplementation. 


\section{A}

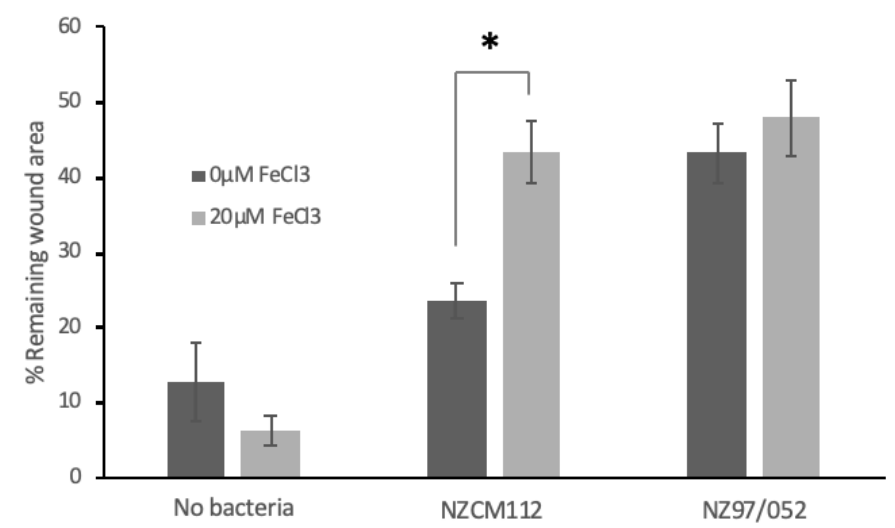

B
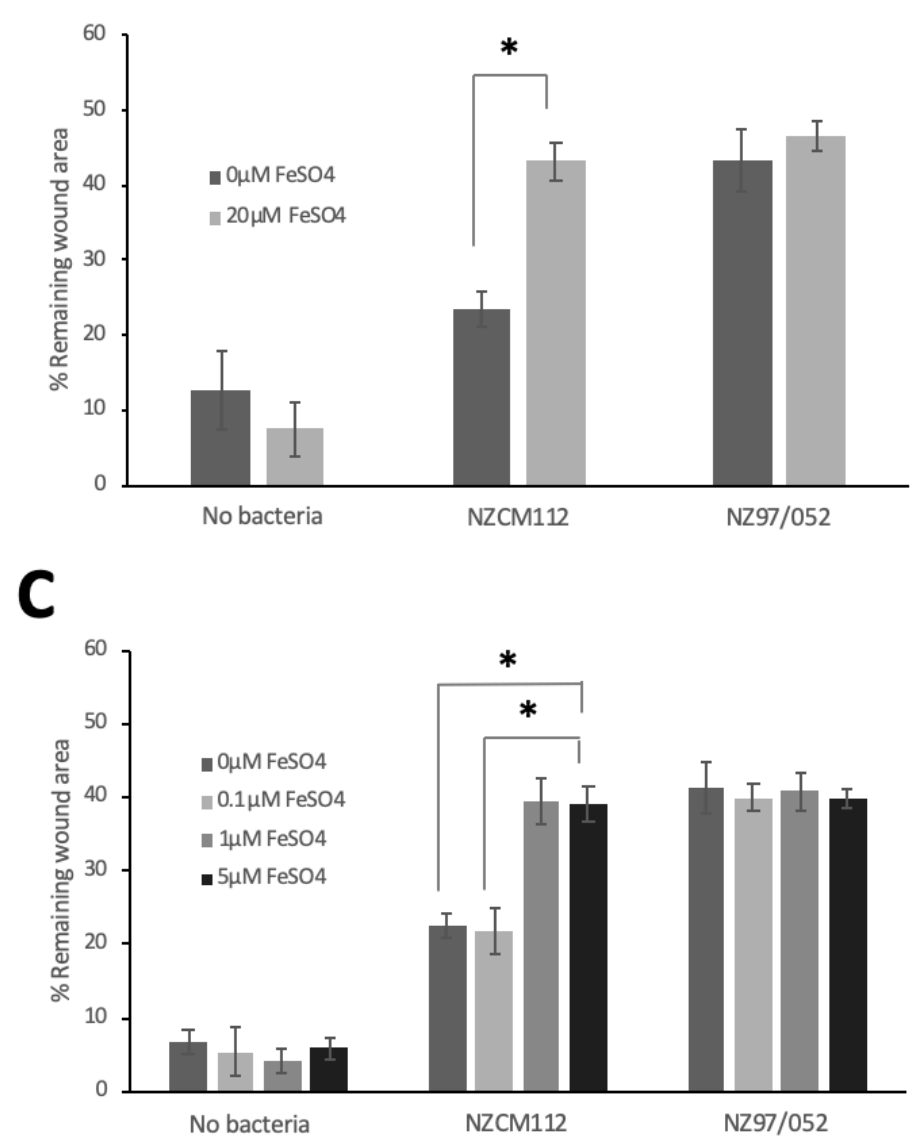

Figure 4.2. Addition of iron ( $>1 \mu \mathrm{M})$ resulted in NZCM112-mediated cell migration inhibition. Supplementation with $20 \mu \mathrm{M} \mathrm{FeCl}_{3}(\mathrm{~A})$ and $\mathrm{FeSO}_{4}$ (B) both increased the ability of the carriage-associated isolate NZCM112 to inhibit wound repair $(p<0.01)$. (C) Iron supplementation does not appear to increase NZCM112-mediated cell migration inhibition in a dose-dependent manner; instead inhibition of cell migration by NZCM112 is seen at $\mathrm{FeSO}_{4}$ concentrations of $1 \mu \mathrm{M}$ or higher. Error bars show standard deviation. Data points are determined from means of eight replicates. P-value $<0.05=*$. 


\subsubsection{Inhibition of re-epithelialisation by other 'carriage-associated' isolates increases in the presence of iron}

In order to determine whether the effect of iron on cell migration inhibition was specific to NZCM112, or whether this phenomenon occurred more widely, iron supplemented cell migration assays were carried out for other carriage-associated isolates (Figure 4.3). The carriage isolates tested were isolates originally obtained from the same household carriage study as the NZ97/052 household group (Simmons et al., 2001). All carriage isolates used for this assay were previously shown to exhibit minimal inhibition of cell migration under normal conditions (Ren \& MacKichan, 2014). Serogroups, strain, and sequence types of these isolates are listed in Chapter Two, Table 2.1. They represent isolates with a serogroup B capsule type and two that were non-groupable (no detectable capsule). NZCM112 is a serogroup C isolate. Although the capsule has previously been shown to not have any impact on the ability of meningococci to inhibit wound repair (Ren \& MacKichan, 2014), these different serogroups were selected to test a diversity of unrelated isolates.

A significant increase in the ability to inhibit cell migration was seen for all of the carriage isolates tested when $20 \mu \mathrm{M}$ of iron sulfate was added to the culture medium ( $p<0.001$ for each isolate, comparing with and without iron addition). The average remaining wound area, as a percent of the initial wound area, increased for all carriage isolates tested by about 2.8 fold. As seen before (Section 4.2.2), addition of iron sulfate to the cell culture medium did not have a significant effect on uninfected migrating $16 \mathrm{HBE}$ cells $(p=0.298)$, which suggests that this effect is not due to the inhibition of $16 \mathrm{HBE}$ cell migration by iron alone. These results were striking, because they suggest the effect of iron on the interaction of NZCM112 with epithelial cells is also seen in a diverse array of carriage isolates that are unable to inhibit cell migration under normal conditions. 


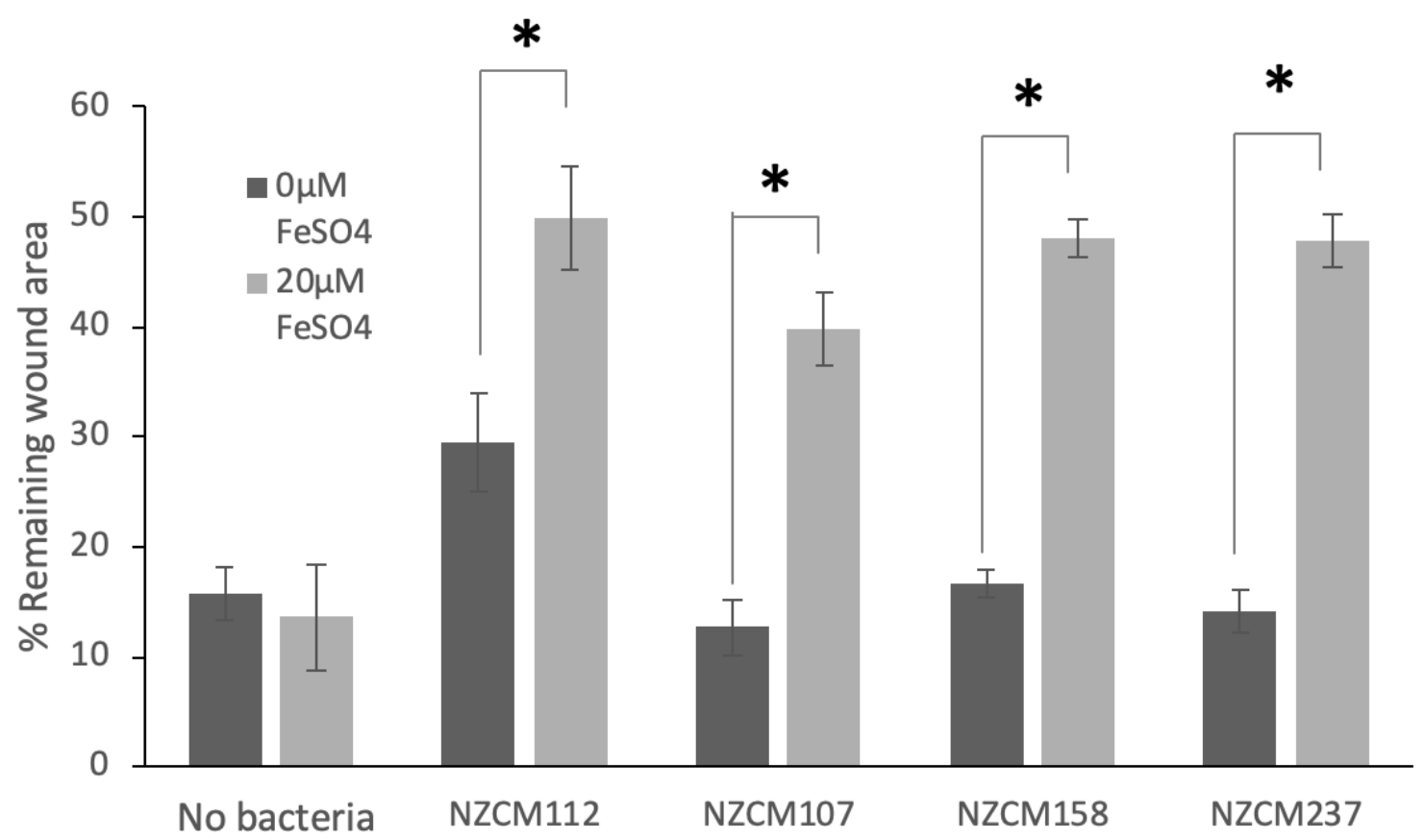

Figure 4.3. Addition of $20 \mu \mathrm{M} \mathrm{FeSO}_{4}$ to the cell culture medium resulted in the ability of multiple carriage isolates to inhibit wound repair. Error bars show standard deviation. Data points are determined from means of eight replicates. * Represents $p<0.05$.

\subsubsection{NZCM112 and NZ97/052 differ in growth rate measured over a time course}

NZ97/052 grew better than NZCM112 under the conditions tested (Chapter Three, Figure 3.4), and the possibility that this was related to the reduced ability of NZCM112 to inhibit cell migration could not be excluded. To address this, growth assays tested whether nutrient supplementation would enable NZCM112 to grow as well as NZ97/052. Iron supplementation greatly increased the ability for carriage associated isolates to inhibit re-epithelialisation (Figure 4.3). Although experiments detailed in Chapter Three suggested that the inhibition of host cell migration is not explained by increased bacterial numbers, experiments were designed to determine whether iron supplementation alters the growth of NZCM112.

Bacterial growth assays were carried out to determine whether meningococcal growth rates were affected by any nutrients. Bacterial growth was measured for NZCM112 and NZ97/052 
isolates in M199 medium, with 10\% FBS. Absorbance was measured over a time course, with and without $20 \mu \mathrm{M}$ iron sulfate $\left(\mathrm{FeSO}_{4}\right)$ supplementation, to determine bacterial growth (Figure 4.4). The assay involved resuspension of overnight cultures of $N$. meningitidis from CBA plates into M199 medium with 10\% FBS to reach an initial optical density at $600 \mathrm{~nm}\left(\mathrm{OD}_{600}\right)$ of 0.2 . Although this assay was completed in the absence of 16HBE cells, serum-supplemented M199 medium was selected to best mimic the conditions of the cell migration assays. The bacterial cultures were grown in a T-75 flask placed flat in a $37^{\circ} \mathrm{C}$ incubator, to prevent oxygen limitation in liquid culture, and without shaking to avoid the formation of aerosols. Cultures were thoroughly mixed before a sample was collected and the $\mathrm{OD}_{600}$ was measured every two hours, until stationary phase was reached.

Optical density measurements were plotted on a semi-logarithmic scale and the bacterial growth rate was calculated from the linear section of the growth curve, which was from two to six hours. The specific growth rate $(k)$ was calculated for each isolate using the following equation:

$$
\mathrm{k}=2.303\left(\log N-\log N_{0}\right) / t
$$

where $t$ represents time elapsed (in hours), and where $N$ and $N_{0}$ are the $O D_{600}$ readings (assumed to be proportional to the numbers of cells) at the final and initial time points respectively. Specific growth rate was calculated for multiple cultures and then averaged $(n=3)$. The average growth rate of the NZ97/052 isolate was $0.195 \mathrm{hr}^{-1}$ and the average growth rate of NZCM112 was a much lower $0.118 \mathrm{hr}^{-1}$. The addition of iron did not significantly impact the growth rate of NZ97/052 (0.195 hr-1 without iron and $0.190 \mathrm{hr}^{-1}$ with $20 \mu \mathrm{M} \mathrm{FeSO}$ ) or NZCM112 (0.118 $\mathrm{hr}^{-1}$ without iron and $0.123 \mathrm{hr}^{-1}$ with $20 \mu \mathrm{M} \mathrm{FeSO}_{4}$ ).

The final $O D_{600}$ of isolates with and without iron sulfate were also compared. At 26 hours, the mean $\mathrm{OD}_{600}$ of $\mathrm{N97/052}$ isolate without $\mathrm{FeSO}_{4}$ was 0.841 and 0.892 in the presence of $20 \mu \mathrm{M}$ $\mathrm{FeSO}_{4}$. This increase was modest but statistically significant $(\mathrm{p}=0.037)$. There was also a small 
but significant increase $(p=0.004)$ when comparing final $\mathrm{OD}_{600}$ of the NZCM112 isolate with (0.466) and without (0.431) $\mathrm{FeSO}_{4}$ supplementation. Although the differences in final $\mathrm{OD}_{600}$ reached significance, the slight variations in starting $\mathrm{OD}_{600}$ should be taken into consideration. Furthermore, at the 8 hour time point, $\mathrm{OD}_{600}$ for the different conditions was very similar. Therefore, these results suggest that the increase in cell migration inhibition by carriage isolates with iron supplementation is not due to increased bacterial numbers.

There are limitations to carrying out growth assays manually, either due to human error or increased chances of contamination. To minimise this, multiple readings were taken and the experiments were carried out multiple times to have at least three triplicates and three technical replicates. An easier and more consistent way of measuring absorbance is on a plate reader, which automatically reads absorbance in each well over a set time. However, due to the dangers of working with live clinical $N$. meningitidis isolates in liquid suspension, a plate reader could not be used in a safe manner, so these absorbance measurements had to be taken manually in a Class II biosafety cabinet. 


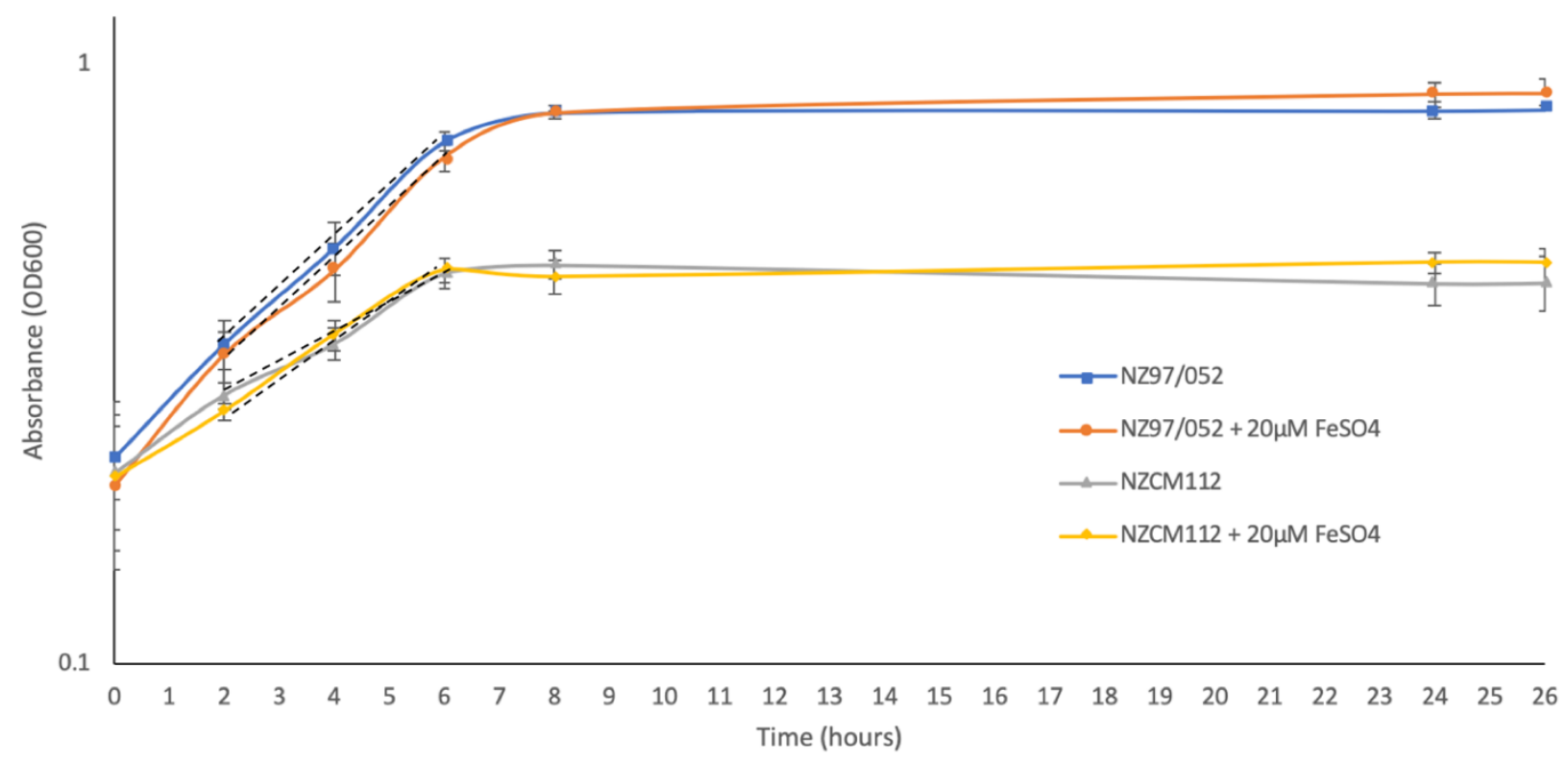

Figure 4.4. The growth rate of NZ97/052 is higher than NZCM112 and iron sulfate supplementation does not impact final concentration reached. Isolates tested were NZ97/052 without iron (blue) or with $20 \mu \mathrm{M} \mathrm{FeSO}$ (orange) and NZCM112 without iron (grey) or with $20 \mu \mathrm{M} \mathrm{FeSO}$ (yellow). The experiment was performed three times with three technical replicates for each condition. Error bars represent standard deviation. Dashed lines show linear section of the curve which was used to calculate the rate of growth.

\subsubsection{Supplementation with iron, zinc, or non-essential amino acids does not affect bacterial growth in NZ97/052 household in the presence of $16 \mathrm{HBE}$ cells}

The growth curve measuring absorbance (Figure 4.4) enabled calculation of the bacterial specific growth rate, but could not detect differences between viable and non-viable bacteria. Therefore, a second growth assay was conducted to measure colony forming units (CFU) at 0 and 16 hours to measure bacterial growth. This assay was carried out in the presence of $16 \mathrm{HBE}$ cells, to mimic the conditions of the respiratory epithelial cell migration assays. To find out whether a difference in cell migration inhibition could be explained by increased bacterial numbers, growth assays were supplemented with the same nutrients used in the Oris cell migration assays (Figure 4.1 and Figure 4.2). Bacterial growth was measured for NZCM112 and NZ97/052 while supplemented with iron sulfate, iron chloride, zinc sulfate, or NEAA (Figure 4.5), to detect whether any nutrients would have a significant impact on bacterial numbers. Iron 
sulfate $\left(\mathrm{Fe}^{2+}\right)$ and iron chloride $\left(\mathrm{Fe}^{3+}\right)$ were both used to detect if different iron oxidation states had different effects. The growth of NZ97/052 remained significantly higher than growth of NZCM112, in terms of final numbers of viable bacteria that were reached $(p<0.001)$ (Figure 4.5). Supplementation with $5 \mu \mathrm{M}$ or $20 \mu \mathrm{M} \mathrm{ZnSO}_{4}$ did not have a significant effect on bacterial growth for either NZCM112 or NZ97/052 (Figure 4.5A). Previous results suggested a modest but significant increase of final concentration of bacteria in M199 medium with the addition of iron (Figure 4.4). However, this effect was not detectable when measuring CFU in the presence of 16HBE cells (Figure 4.5B). This is presumably because the effect of iron supplementation on growth was modest, and the variation between replicates was larger when measuring CFU than when measuring optical density. Supplementation with $5 \mu \mathrm{M}$ or $20 \mu \mathrm{M}$ NEAA also did not have any significant effect on bacterial growth for either isolate (Figure $4.5 \mathrm{C}$ ). These results suggest the nutrients were not growth-limiting for the bacteria in the conditions tested. The number of bacterial generations $(n)$ were calculated using the following equation:

$$
n=3.3\left(\log N-\log N_{0}\right)
$$

where $\mathrm{N}$ was the final number of viable bacteria, and $\mathrm{N}_{0}$ was the initial number of bacteria. The number of bacterial generations for NZ97/052 over 16 hours was calculated to be 8.4 , while the number of generations for NZCM112 was 6.2. These results confirm observations that NZCM112 enters stationary phase at a lower cell density, relative to NZ97/052 (Figure 4.4).

These results supported the growth assays completed in Chapter 3 (Figure 3.4) and confirmed NZ97/052 grows to higher numbers than NZCM112. The differences in growth between NZ97/052 and NZCM112 were clearly not altered by iron supplementation. Iron was the only supplement which conferred the ability of NZCM112 to inhibit cell migration, however it did not substantially alter bacterial growth (Figure 4.4, Figure 4.5). Altered bacterial growth, or increased bacterial numbers, is therefore not likely to account for the increase in inhibition of re-epithelisation observed when NZCM112 infects $16 \mathrm{HBE}$ cells in the presence of iron. 


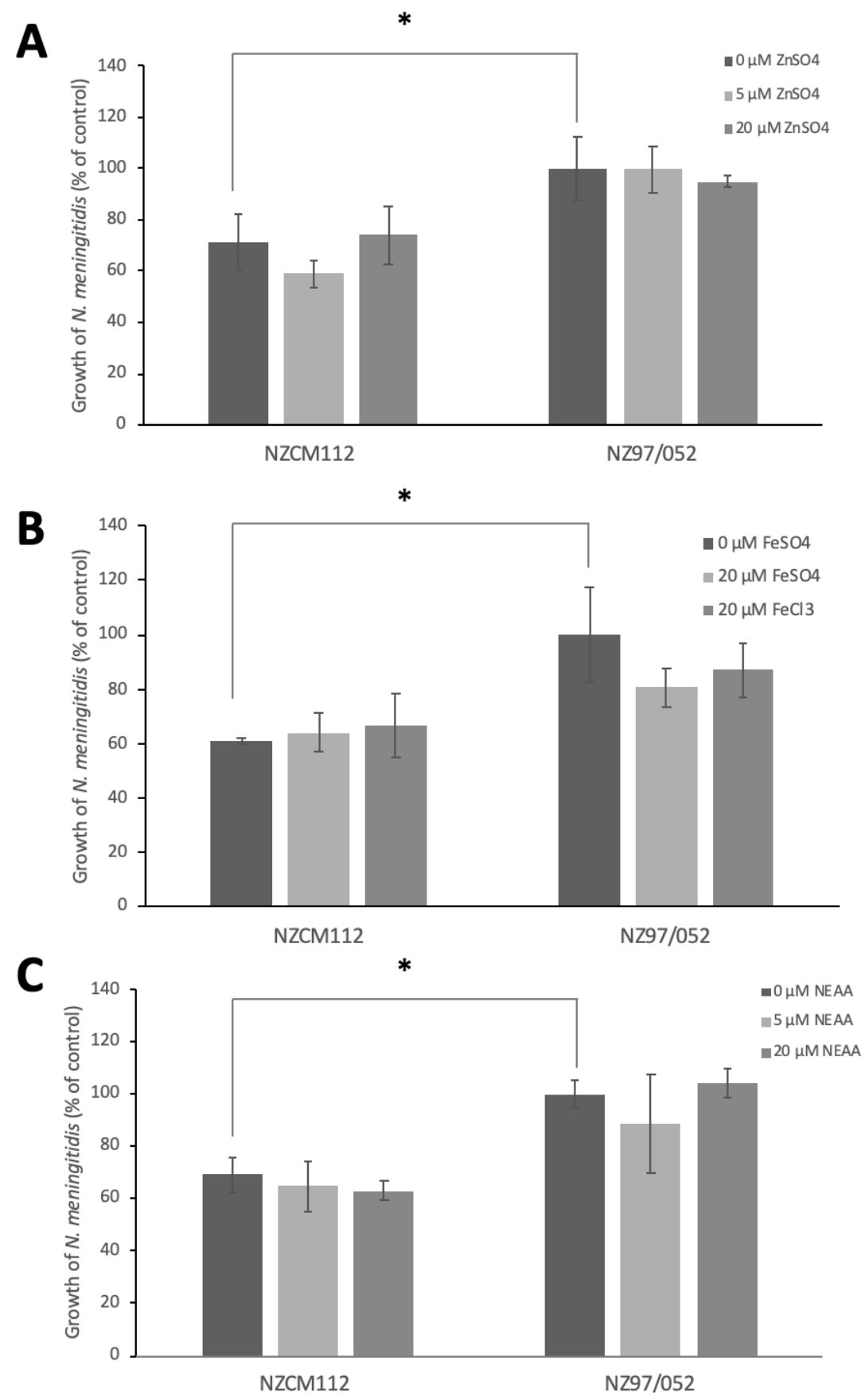

Figure 4.5. Nutrient supplementation did not significantly alter bacterial growth in the presence of host cells. NZ97/052 grew to higher numbers than NZCM112. A viable cell count was completed to assess the growth of NZCM112 and NZ97/052 isolates with (A) $0 \mu \mathrm{M}, 5 \mu \mathrm{M}$ and $20 \mu \mathrm{M}$ zinc sulfate supplementation, (B) $20 \mu \mathrm{M}$ iron sulfate or iron chloride supplementation, and (C) $0 \mu \mathrm{M}, 5 \mu \mathrm{M}$ and $20 \mu \mathrm{M}$ non-essential amino acids (NEAA) supplementation. This assay compared viable bacterial cells (CFU) after 16 hours of co-incubation to input CFU, normalised relative to the growth of NZ97/052 isolate. Each experiment was performed three times with three replicates. ${ }^{*}$ Represents $p$ value $<0.05$. 


\subsubsection{Association of NZCM112 with 16HBE cells increases in the presence of iron}

Iron was shown to increase inhibition of cell migration by NZCM112 (Figure 4.3). Experiments were designed to determine whether iron supplementation also impacts bacterial adhesion to host cells, as results from Chapter Three (Section 3.2.5) suggested adhesion to respiratory epithelial cells corresponded to the inhibition of cell migration. The effect of iron supplementation on the degree of cell association was measured for NZ97/052 and NZCM112. The cell culture supernatants were supplemented with $20 \mu \mathrm{M} \mathrm{FeSO}_{4}$, and incubated for one hour (Figure 4.6A). Interestingly, iron sulfate supplementation increased the mean percent of cell-associated NZCM112 from $1.5 \%$ to $2.8 \%$ of the initial inoculum ( $p<0.001$ ). No difference in cell association was seen in NZ97/052 isolate with or without iron supplementation $(4.9 \%$ and $4.8 \%$, respectively). These results were intriguing as iron supplementation was also shown to increase inhibition of cell migration by NZCM112 only (Figure 4.3). This provides further evidence that inhibition of cell migration may be linked to cell adhesion. The difference in association cannot likely be attributed to a difference in bacterial growth, as after one hour growth differences between NZCM112 and NZ97/052 are not significantly different (Figure 4.4).

A gentamicin protection assay was also carried out in the presence of $20 \mu \mathrm{M} \mathrm{FeSO}_{4}$, to determine whether iron supplementation had an impact on the invasion of human host cells (Figure 4.6B). The results were analogous to those of the cell association assay; no significant difference was seen in the percentage of intracellular NZ97/052, with or without iron sulfate supplementation (0.34\% and $0.32 \%$ respectively). However, supplementation with $20 \mu \mathrm{M} \mathrm{FeSO}$ increased the percentage of intracellular carriage-associated isolate NZCM112 CFU, from 0.13\% to $0.20 \%$ of the initial inoculum. This difference was statistically significant $(p=0.027)$. This result can likely be explained due to the increased number of bacteria adhered to the $16 \mathrm{HBE}$ cells. 


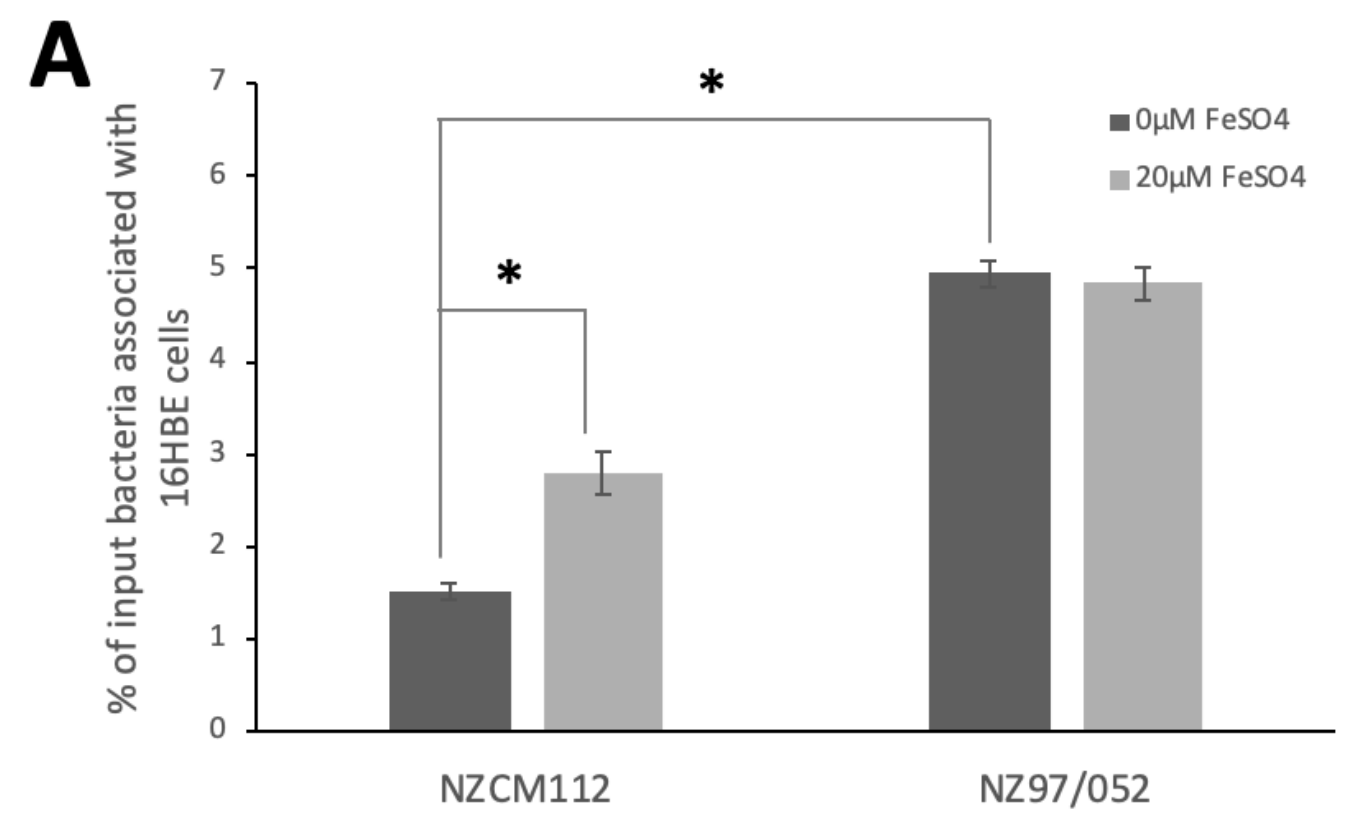

B

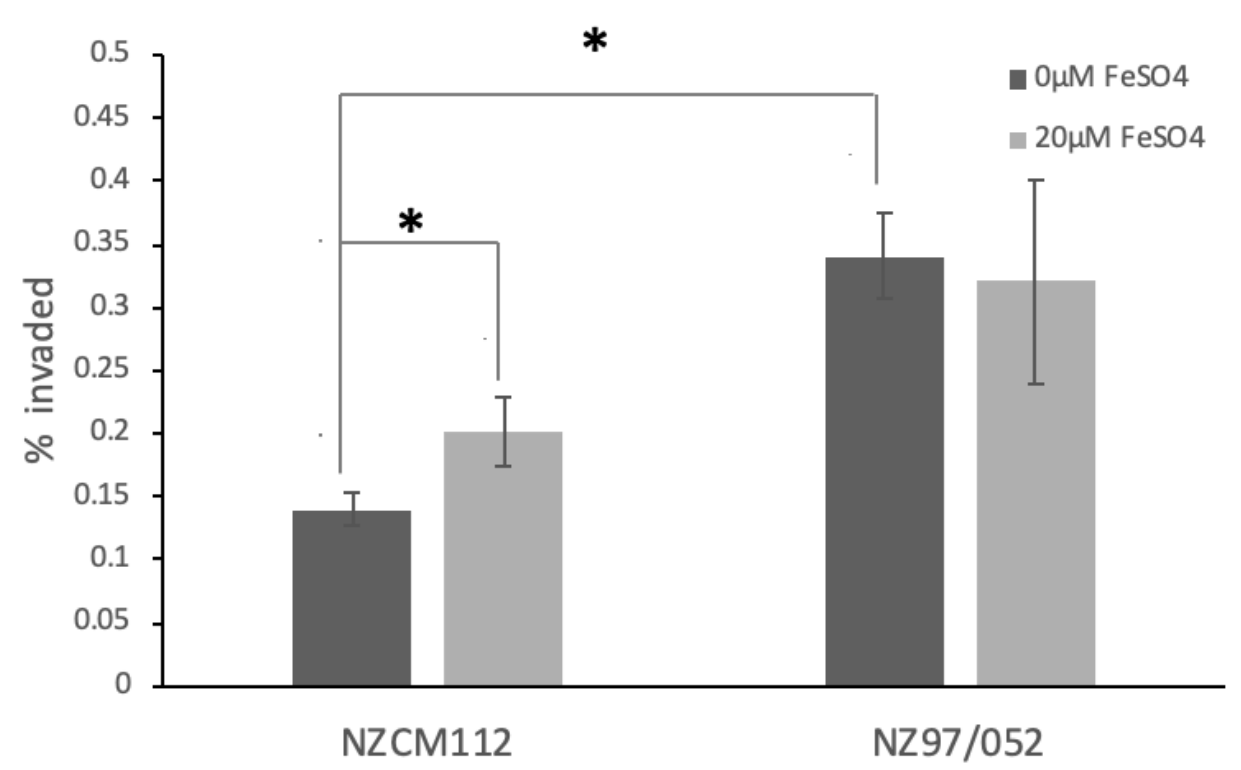

Figure 4.6. Iron supplementation increases association to and invasion of 16HBE cells by NZCM112. (A)

Supplementation of bacteria-cell co-culture with $20 \mu \mathrm{M}$ of $\mathrm{FeSO}_{4}$ resulted in a significant increase in cell association for the NZCM112 isolate, but not the NZ97/052 isolate (B) Supplementation of bacteria-cell co-culture with $20 \mu \mathrm{M}$ $\mathrm{FeSO}_{4}$ caused a significant increase in cell invasion for NZCM112 isolate only. Experiments were performed in triplicate, three independent times. Error bars represent standard deviation. ${ }^{*}$ Represents $p$-value $<0.05$ 


\subsection{Discussion}

Inhibition of cell migration by $N$. meningitidis may be a route by which virulent isolates cause invasive disease, however it is unknown how some isolates prevent host cell re-epithelisation. It is possible a phase variable gene is involved, however many phase variable genes and surface factors that are known to interact with host cells have already been excluded (Ren \& MacKichan, 2014). Nutritional virulence is a crucial determinant of invasive capability, particularly the uptake of nutrients in restricted conditions, and it has been established that metabolic differences exist between carriage and disease meningococcal isolates (Schoen et al., 2014). It was hypothesised that invasive isolates of $N$. meningitidis, which were highly effective at inhibiting wound repair, were starving respiratory epithelial cells by consuming nutrients in the medium essential for host cell migration. The goal of this chapter was to explore whether bacterial metabolism and nutrient uptake could be a mechanism of inhibition of cell migration by disease-associated $N$. meningitidis isolates. It was expected that supplementing the medium with nutrients might be able to 'overcome' the ability for the disease-associated bacteria to inhibit host cell migration.

Cell migration assays were supplemented with various nutrients important for bacterial growth or cell migration. This included single additives, such as zinc sulfate $\left(\mathrm{ZnSO}_{4}\right)$, iron sulfate $\left(\mathrm{FeSO}_{4}\right)$, and iron chloride $\left(\mathrm{FeCl}_{3}\right)$ as well as pooled supplements such as non-essential amino acids (NEAA). Uninfected cell migration controls were included in each assay. Supplementation of Oris cell migration assays with zinc and NEAA did not cause any noticeable change in cell migration inhibition by any meningococcal isolates. Supplementation with iron did not have any effect on the migration $16 \mathrm{HBE}$ cells infected with disease-associated isolate NZ97/052. These results suggested that the hypothesis, that disease-associated isolate NZ97/052 was consuming nutrients in the medium necessary for cell migration, was incorrect.

However, unexpected and striking results were seen when migrating $16 \mathrm{HBE}$ cells, infected with the carriage-associated isolate NZCM112, were supplemented with iron. Supplementation with 
iron resulted in increased NZCM112-mediated inhibition of cell migration, to a rate where it was indistinguishable from the disease-associated isolate, NZ97/052. Iron sulfate did not significantly alter cell migration of uninfected $16 \mathrm{HBE}$ cells, so this cannot be due to iron toxicity or iron-mediated inhibition of host cell migration. The effect of iron supplementation of NZCM112 does not appear to be dose-dependent, and was independent of iron oxidation state, as it was observed with supplementation with both iron sulfate $\left(\mathrm{Fe}^{2+}\right)$ and iron chloride $\left(\mathrm{Fe}^{3+}\right)$. Interestingly, this effect was not specific to NZCM112, as iron sulfate supplementation of multiple carriage isolates from different serogroups ( $B, C$ and non-groupable) and sequence types (ST-8, ST-2154, ST-178 and an unknown ST) resulted in all of them gaining the ability to inhibit cell migration. This suggests the effect is mediated by a meningococcal factor that is common among carriage-associated isolates.

Transition metal levels must be carefully controlled by bacteria (Guilhen et al., 2013) as these can be toxic at high concentrations due to their catalytic activity (Hood \& Skaar, 2012). Therefore, nutrient supplementation may be beneficial or detrimental at different concentrations. Growth assays were carried out with iron, zinc and non-essential amino acid supplementation. These were completed to ensure the nutrients did not have a detrimental effect on the bacteria and to further investigate if differences in growth rate could explain variations in cell migration inhibition. None of the supplemented nutrients had a significant effect on the growth rate of NZ97/052 or NZCM112. Addition of iron did not lead to increased bacterial numbers, although it did result in NZCM112 gaining the ability to inhibit cell migration. Association and invasion assays were also carried out with iron supplementation to detect whether iron also has an impact on association to respiratory epithelial cells. Iron caused increased cell association of NZCM112 only.

The disease-associated isolate NZ97/052 inhibits cell migration and associates with epithelial cells without iron supplementation, and adding iron does not impact this phenotype. However, when iron was added to NZCM112 it not only increased the ability to inhibit cell migration but also improved adherence. These results implicate iron in an important role in cell migration 
inhibition, though this is not mediated by limitation of iron to host cells. Free iron may result in increased gene expression, in the carriage isolates, of a gene that facilitates inhibition of host cell wound repair. This implies alteration of a meningococcal factor on the surface of the bacteria that also mediates adhesion. These results cannot identify whether the diseaseassociated isolate NZ97/052 lacks this iron-induced gene, or whether its impact is masked by another factor that is made constitutively.

This hypothesised surface factor is unlikely to be involved in iron scavenging from the host, as many iron uptake proteins are usually turned on under low iron conditions (Grifantini et al., 2003; Jordan \& Saunders, 2009). Instead, the carriage isolate factor appears to be switched on in high iron conditions. Because its effect becomes apparent when the bacteria and cells are supplemented with ferrous or ferric iron, the factor could be a transporter that plays a role in the acquisition of iron that is not in a complex with host proteins, such as haem or transferrin.

A previous study by Grifantini et al showed that addition of iron to N. meningitidis serogroup B strain MC58 caused upregulation of 153 genes (2003). Of these, the candidate gene(s) involved in cell association and migration inhibition are predicted to be surface exposed, enabling them to mediate adhesion. One interesting candidate is outer membrane protein NspA (Neisseria surface protein A), which is a highly conserved surface protein of unknown function, and has been shown to be highly upregulated in the presence of iron (Grifantini et al., 2003; Shaik et al., 2007). Other surface exposed genes which have been shown to be upregulated in the presence of iron include pan1, siaD, lipA, lipB, murE, and mraY (Grifantini et al., 2003). All of these genes, and their potential impact on cell migration or adherence, would be interesting to investigate further. Iron had the same effect on cell migration inhibition by carriage isolates from diverse lineages, suggesting the meningococcal factor that impacts cell migration is widely conserved.

Additional experiments to identify this iron-inducible meningococcal factor could include transcriptome profiling experiments to assess the effect of iron supplementation on multiple carriage isolates. This approach could allow identification of candidate genes which are turned 
on in the presence of iron, which could be deleted in NZCM112. Resulting mutants could be tested for the ability to increase cell migration inhibition and adherence when supplemented with iron. These experiments were not carried out in this project, as the focus was instead on the identification of the meningococcal factor responsible for adherence and inhibition of wound repair in NZ97/052. However, it is important to keep in mind that there are likely additional factors to be discovered.

There are intriguing potential clinical considerations for this finding. Iron has been well established to be important for bacterial virulence. Some clinical observations support this; patients with haemochromatosis have shown increased susceptibility to infection by certain pathogens, including E. coli, Vibrio cholerae, and Listeria monocytogenes (Christopher, 1985; Fernandez et al., 2000; Manso et al., 1997). However there have been no clinical trials to evaluate patients with infections and the potential negative impact of taking iron supplementation, which could be an interesting avenue to explore. Based on findings from this chapter, it could be speculated that taking an iron supplement may increase the likelihood for a 'carriage-associated' isolate, which would normally be carried asymptomatically, to cause invasive disease, provided it also carries virulence factors, such as a capsule, that enable multiplication in the bloodstream.

\subsection{Summary}

The effect of nutrient supplementation on various in vitro assays disproved the hypothesis that disease-associated isolate NZ97/052 was inhibiting host cell wound repair by consuming nutrients in the medium necessary for re-epithelisation of host cells. Unexpectedly, iron availability promoted the phenotype of cell migration inhibition and cell association in carriage associated isolate NZCM112, and other unrelated carriage isolates. This surprising effect suggests an important role of iron in wound repair inhibition by N. meningitidis. These results implicated a gene involved with iron uptake or an iron-regulated gene. Iron may be 'switching on' a gene in carriage associated bacteria which is involved in host cell wound repair. 


\section{Identification of the meningococcal factor responsible for respiratory epithelial cell wound repair inhibition}

\subsection{Introduction}

Damage to the nasopharynx and respiratory epithelium increases the likelihood that $N$. meningitidis can cross the epithelial layer, reach deeper tissues, and cause invasive disease (Coen et al., 2006; Stephens et al., 2007; Stephens et al., 1983). Inhibition of respiratory epithelial cell wound repair may be an important phenotype related to the initiation of invasive disease, as this prolongs the opportunity for colonising $N$. meningitidis to enter the bloodstream (Ren \& MacKichan, 2014). The goal of this chapter was to identify the meningococcal factor responsible for the inhibition of host cell migration.

The NZ97/052 household contains three isolates which were characterised in Chapter Three. These three isolates are indistinguishable by standard laboratory typing, and all belong to the strain type C:2b:P1.2,5 (Simmons et al., 2001). The isolates in this household differed in the phenotype of wound repair inhibition; NZ97/052 and NZCM111 were effective at inhibiting cell migration of $16 \mathrm{HBE}$ cells, whereas NZCM112 was not. Analysing closely related household bacterial isolates can be a useful tool for discovering genes responsible for a particular phenotypic trait, as it can allow for easier identification of candidate meningococcal transcripts than comparing many meningococcal genomes at once. Whole genome-sequencing of the NZ97/052 household isolates was utilised to identify variants which differ between bacterial isolates. However, even closely related isolates can vary widely in the number of single nucleotide polymorphisms (SNPs). A study by Ren et al. used whole-genome sequencing to identify SNPs in household meningococcal isolates, which demonstrated that the number of variants between household isolates could range from as little as nine to as many as 210 . The later was a case where an epidemic strain acquired $\sim 5 \mathrm{~kb}$ of DNA from the commensal Neisseria polysaccharae while in the nasopharynx (Ren et al., 2017). To further narrow down the list of candidate genes, NZ97/052 and NZCM112 were analysed by RNA-seq profiling. To assess if a 
particular gene is involved in a phenotypic trait, candidate genes can be deleted by allelic replacement. If a gene deletion mutant differs in phenotype to the wild-type parent strain, this suggests the deleted gene is responsible for that particular phenotype.

\subsubsection{Aims of this chapter}

The aim of this chapter was to examine genomic and transcriptomic differences between disease- and carriage-associated isolates of the NZ97/052 household, and to identify candidate genes responsible for cell migration inhibition. Transcriptome analysis identified changes in expression between isolates and genomic comparisons identified nucleotide variants. Genome and transcriptome data were cross-referenced to the current literature to identify which genes could confer the ability to inhibit cell migration. Top candidate genes were deleted by allelic replacement from the disease-associated isolate NZ97/052. Cell migration assays were used to compare gene deletion mutants to the original parent isolate. The aim of these assays was to determine whether the absence of the candidate genes abrogated the ability of the diseaseassociated isolate to inhibit cell migration. This chapter aimed to identify the meningococcal factor responsible for wound repair inhibition, and to use mutagenesis to transform a virulent disease-associated isolate into a more carriage-like isolate, by revoking the ability to inhibit wound repair.

\subsection{Results}

\subsubsection{Genome comparisons between isolates in the NZ97/052 household}

Genomic analysis of relatedness among household isolates can provide insights and explanations as to why certain genes are more highly expressed in one isolate over another. Genome sequencing was completed to identify coding regions missing from the NZCM112 isolate which could be responsible for the reduction in cell migration inhibition. The goal was to identify the bacterial factor essential for inhibition of host cell migration. 
Genomic DNA was purified from the three N. meningitidis isolates, and whole-genome sequencing was performed by New Zealand Genomics Limited (NZGL). Isolates were sequenced by Illumina MiSeq using paired-end sequencing. Reads of NZ97/052, NZCM112, and NZCM111 meningococcal genomes were aligned against the $N$. meningitidis NMBG2136 reference genome, which belongs to ST-8, serogroup B isolate (RefSeq genome NC_017513.1) (Budroni et al., 2011). NMBG2136 was selected as a reference genome since it has the same sequence type as the NZ97/052 household, even though this isolate belongs to serogroup B instead of serogroup C. Nucleotide variants were identified using Freebayes 1.0 workflow (Garrison \& Marth, 2012), Bowtie 2.1.0 (Langmead \& Salzberg, 2012), and GATK 3.3-0 UnifiedGenotyper (McKenna et al., 2010). Genome comparisons were completed for the isolates in the NZ97/052 household to identify differences in the genome sequence between NZ97/052 and NZCM111, which both inhibit wound repair, and NZCM112, which does not. Variants were analysed in "clumped" format, where variants close to each other were presented as one change; this method ensures that changes within a codon are annotated correctly. A total of 49 clumped variants were identified, where nucleotide differences occurred between the household isolates (Table 5.1). Intergenic variants, classified as those over $100 \mathrm{bp}$ away from the nearest predicted coding region, made up 20 of the polymorphisms detected. Nine variants were fewer than $100 \mathrm{bp}$ upstream of an annotated transcript, and one was fewer than $100 \mathrm{bp}$ downstream. Within coding regions, four variants caused synonymous changes, where the encoded amino acid was not changed. Nine variants caused missense changes, four variants were predicted to cause a frameshift, one was classified as an in-frame deletion and one was an in-frame insertion. 
Table 5.1. Number and types of nucleotide variants which occur between isolates in the NZ97/052 household.

\begin{tabular}{ll}
\hline Variant type & Count \\
\hline Intergenic & 20 \\
Upstream & 9 \\
Downstream & 1 \\
Synonymous & 4 \\
In-frame insertion & 1 \\
In-frame deletion & 1 \\
Missense & 9 \\
Frameshift & 4 \\
\hline Total variants & 49 \\
\hline
\end{tabular}

The variants of greatest interest were those that were found only in NZCM112, but not NZ97/052 or NZCM111 (Table 5.2). There were 17 variants which met this criteria. Two variants were synonymous and two were missense, neither of which were likely to result in major phenotypic differences. There were eight upstream and four intergenic variations. The upstream variants were primarily clustered in front of two genes, trmA and NMBG2136_1658. These variant clusters occurred in a space of fewer than 50 nucleotides. This small degree of difference suggested that a recombination event had occurred, although it is unknown which isolates contained the original and which contained the recombination sequence. The significance of variants in the non-coding regions is not entirely understood, and the possibility that these variants could affect gene expression of nearby coding regions cannot be excluded.

One variant of particular interest was the replacement of a six-base sequence, CGGGGG, by a five-base sequence, CAGCA. This resulted in a frameshift in $h p u A$, which occurred in the carriage-associated isolate NZCM112 only. The variant in hpuA is the only one of the 49 total variants that resulted in the loss of expression of a protein. Furthermore, this loss occurred only in NZCM112, but not NZ97/052 or NZCM111. Therefore, hpuA was identified as a candidate gene. 
The other frameshift variants found in the NZ97/052 household were in NMBG2136_1563, which encodes a putative deoxyribonuclease, however these were not considered as candidates, as the variants occurred in both NZ97/052 and NZCM112 and could not account for the difference in wound repair inhibition between these isolates. Furthermore, the NZ97/052 and NZCM112 allele of NMBG2136_1563 was entered into the PubMLST allele database (Jolley \& Maiden, 2014) and was identical to alleles in unrelated N. meningitidis isolates. This suggested these variants resulted from a crossover event with another carriage isolate. Although there are four frameshift mutations in this gene, they essentially cancel each other out and do not result in a premature stop codon. As this variant is identical to alleles found in other isolates, it is likely to be functional. These frameshift variants demonstrate the genome plasticity seen in N. meningitidis.

Table 5.2. Variants among the NZ97/052 household.

\begin{tabular}{|c|c|c|c|c|c|c|}
\hline $\begin{array}{l}\text { Position } \\
\text { (on } \\
\text { NMBG2136 } \\
\text { genome) }\end{array}$ & $\begin{array}{l}\text { Reference } \\
\text { sequence } \\
\text { NMBG } 2136\end{array}$ & NZ97/052 & NZCM111 & NZCM112 & Locus & $\begin{array}{l}\text { Type of } \\
\text { variant }\end{array}$ \\
\hline 46951 & $A$ & G & $A$ & G & $\begin{array}{l}\text { NZBM2136_0045- } \\
\text { NMBG2136_0046 }\end{array}$ & Intergenic \\
\hline 46958 & $A$ & G & $A$ & $A$ & $\begin{array}{l}\text { NZBM2136_0045- } \\
\text { NMBG2136_0046 }\end{array}$ & Intergenic \\
\hline 46970 & $\mathrm{~T}$ & C & $\mathrm{T}$ & $\mathrm{T}$ & $\begin{array}{l}\text { NZBM2136_0045- } \\
\text { NMBG2136_0046 }\end{array}$ & Intergenic \\
\hline $115414 *$ & $\mathrm{C}$ & $T$ & $T$ & $\mathrm{C}$ & NMBG2136_0108 & Missense \\
\hline $499812 *$ & C & $T$ & $T$ & $\mathrm{C}$ & NMBG2136_0471 & Synonymous \\
\hline 525233 & GCACCA & ACATCC & GCACCA & ACATCC & $\begin{array}{l}\text { NMBG2136_0489- } \\
\text { NMBG2136_0490 }\end{array}$ & Intergenic \\
\hline 592932 & C & $\mathrm{T}$ & C & $\mathrm{C}$ & $\begin{array}{l}\text { NMBG2136_0553- } \\
\text { NMBG2136_0554 }\end{array}$ & Intergenic \\
\hline 592941 & $\mathrm{C}$ & $T$ & C & $\mathrm{T}$ & $\begin{array}{l}\text { NMBG2136_0553- } \\
\text { NMBG2136_0554 }\end{array}$ & Intergenic \\
\hline 592951 & ATCCC & АТССТССС & ATCCC & АТССТССС & $\begin{array}{l}\text { NMBG2136_0553- } \\
\text { NMBG2136_0554 }\end{array}$ & Intergenic \\
\hline 592981 & G & G & $\mathrm{T}$ & $\mathrm{T}$ & $\begin{array}{l}\text { NMBG2136_0553- } \\
\text { NMBG2136_0554 }\end{array}$ & Intergenic \\
\hline 593023 & C & C & G & G & $\begin{array}{l}\text { NMBG2136_0553- } \\
\text { NMBG2136_0554 }\end{array}$ & Intergenic \\
\hline
\end{tabular}




\begin{tabular}{|c|c|c|c|c|c|c|}
\hline $\begin{array}{l}\text { Position } \\
\text { (on } \\
\text { NMBG2136 } \\
\text { genome) } \\
\end{array}$ & $\begin{array}{l}\text { Reference } \\
\text { sequence } \\
\text { NMBG2136 }\end{array}$ & NZ97/052 & NZCM111 & NZCM112 & Locus & $\begin{array}{l}\text { Type of } \\
\text { variant }\end{array}$ \\
\hline 593083 & $\mathrm{~T}$ & $\mathrm{C}$ & $\mathrm{T}$ & $\mathrm{C}$ & $\begin{array}{l}\text { NMBG2136_0553- } \\
\text { NMBG2136_0554 }\end{array}$ & Intergenic \\
\hline $786237 *$ & $\mathrm{G}$ & G & $\mathrm{G}$ & A & NMBG2136_0758 & Missense \\
\hline 891302 & $\mathrm{~A}$ & $\mathrm{G}$ & $\mathrm{A}$ & $\mathrm{G}$ & NMBG2136_0882 & Synonymous \\
\hline 891363 & $\mathrm{C}$ & $\mathrm{T}$ & $\mathrm{C}$ & $\mathrm{C}$ & NMBG2136_0882 & Missense \\
\hline 910821 & $\mathrm{~T}$ & G & $\mathrm{T}$ & $\mathrm{T}$ & $\begin{array}{l}\text { NMBG2136_0898- } \\
\text { NMBG2136_0899 }\end{array}$ & Intergenic \\
\hline 1053414 & $\begin{array}{l}\text { TGGCTGT } \\
\text { AGAGATG }\end{array}$ & CGG & $\begin{array}{l}\text { TGGCTGT } \\
\text { AGAGATG }\end{array}$ & CGG & NMBG2136_1043 & Downstream \\
\hline $1145829 *$ & $\mathrm{C}$ & $\mathrm{C}$ & $\mathrm{C}$ & $A$ & $\begin{array}{l}\text { NMBG2136_1137- } \\
\text { NMBG2136_1138 }\end{array}$ & Intergenic \\
\hline 1184195 & $\mathrm{G}$ & C & $\mathrm{G}$ & C & NMBG2136_1170 & Missense \\
\hline $1503614 *$ & CCA & TTC & TTC & CAA & $\begin{array}{l}\text { NMBG2136_1422- } \\
\text { NMBG2136_1423 }\end{array}$ & Intergenic \\
\hline $1649365 *$ & $\begin{array}{l}\text { CACTGCA } \\
\text { AAA }\end{array}$ & $\begin{array}{l}\text { TGCCTCGC } \\
\text { CG }\end{array}$ & $\begin{array}{l}\text { TGCCTCGC } \\
\text { CG }\end{array}$ & $\begin{array}{l}\text { CACTGCA } \\
\text { AAA }\end{array}$ & $\begin{array}{l}\text { NMBG2136_1555 } \\
\text { (trmA) }\end{array}$ & Upstream \\
\hline $1649382 *$ & $\mathrm{CCC}$ & $\mathrm{ACT}$ & ACT & $\mathrm{CCC}$ & $\begin{array}{l}\text { NMBG2136_1555 } \\
\text { (trmA) }\end{array}$ & Upstream \\
\hline $1649388 *$ & TAC & TGACTGC & TGACTGC & TAC & $\begin{array}{l}\text { NMBG2136_1555 } \\
\text { (trmA) }\end{array}$ & Upstream \\
\hline $1649393 *$ & GCATATA & ССССАТСА & ССССАТСА & GCATATA & $\begin{array}{l}\text { NMBG2136_1555 } \\
\text { (trmA) }\end{array}$ & Upstream \\
\hline $1649400 *$ & $\begin{array}{l}\text { ACCCGTTT } \\
\text { TCCTCCAA }\end{array}$ & $\begin{array}{l}\text { ATGACGG } \\
\text { ACGGG }\end{array}$ & $\begin{array}{l}\text { ATGACGG } \\
\text { ACGGG }\end{array}$ & $\begin{array}{l}\text { ACCCGTTT } \\
\text { TCCTCCAA }\end{array}$ & $\begin{array}{l}\text { NMBG2136_1555 } \\
\text { (trmA) }\end{array}$ & Upstream \\
\hline 1657564 & GG & GATG & GG & GATG & NMBG2136_1563 & Frameshift \\
\hline 1657567 & $A C$ & $\begin{array}{l}\text { ATAACCTA } \\
\text { CC }\end{array}$ & $A C$ & $\begin{array}{l}\text { ATAACCTA } \\
\text { CC }\end{array}$ & NMBG2136_1563 & Frameshift \\
\hline 1657569 & GGAG & GTCCCAA & GGAG & GTCCCAA & NMBG2136_1563 & $\begin{array}{l}\text { In frame } \\
\text { insertion }\end{array}$ \\
\hline 1657577 & CTG & GCA & CTG & GCA & NMBG2136_1563 & Missense \\
\hline 1657591 & $\mathrm{~T}$ & $\mathrm{C}$ & $\mathrm{T}$ & C & NMBG2136_1563 & Synonymous \\
\hline 1657599 & $\begin{array}{l}\text { TCGCGGC } \\
\text { GATTTCCG } \\
\text { CCGCGCC } \\
\text { GGTA }\end{array}$ & $\begin{array}{l}\text { CCTTGGCA } \\
\text { ATCTCTTC } \\
\text { CACACAG } \\
\text { GTT }\end{array}$ & $\begin{array}{l}\text { TCGCGGC } \\
\text { GATTTCCG } \\
\text { CCGCGCC } \\
\text { GGTA }\end{array}$ & $\begin{array}{l}\text { CCTTGGCA } \\
\text { ATCTCTTC } \\
\text { CACACAG } \\
\text { GTT }\end{array}$ & NMBG2136_1563 & Missense \\
\hline 1657630 & $\begin{array}{l}\text { TATCTGCG } \\
\text { CG }\end{array}$ & $\begin{array}{l}\text { GATTTCCG } \\
\text { CA }\end{array}$ & $\begin{array}{l}\text { TATCTGCG } \\
\text { CG }\end{array}$ & $\begin{array}{l}\text { GATTTCCG } \\
\text { CA }\end{array}$ & NMBG2136_1563 & Missense \\
\hline 1657645 & TTCG & CTTT & TTCG & CTTT & NMBG2136_1563 & Missense \\
\hline 1657653 & $\mathrm{TC}$ & TACC & $\mathrm{TC}$ & TACC & NMBG2136_1563 & Frameshift \\
\hline
\end{tabular}




\begin{tabular}{|c|c|c|c|c|c|c|}
\hline $\begin{array}{l}\text { Position } \\
\text { (on } \\
\text { NMBG2136 } \\
\text { genome) }\end{array}$ & $\begin{array}{l}\text { Reference } \\
\text { sequence } \\
\text { NMBG2136 }\end{array}$ & NZ97/052 & NZCM111 & NZCM112 & Locus & $\begin{array}{l}\text { Type of } \\
\text { variant }\end{array}$ \\
\hline 1657656 & GAAG & GG & GAAG & GG & NMBG2136_1563 & Frameshift \\
\hline 1746502 & $\begin{array}{l}\text { ACTTTTTT } \\
\text { A }\end{array}$ & $\begin{array}{l}\text { GCTTTTTT } \\
\text { TA }\end{array}$ & $\begin{array}{l}\text { ACTTTTTT } \\
\text { A }\end{array}$ & $\begin{array}{l}\text { GCTTTTTT } \\
\text { TA }\end{array}$ & NMBG2136_1640 & Upstream \\
\hline 1747219 & C & $\mathrm{T}$ & C & $\mathrm{C}$ & $\begin{array}{l}\text { NMBG2136_1640- } \\
\text { NMBG2136_1641 }\end{array}$ & Intergenic \\
\hline 1767864 * & A & A & $A$ & C & NMBG2136_1658 & Upstream \\
\hline $1767871 *$ & $\mathrm{~T}$ & $\mathrm{~T}$ & $\mathrm{~T}$ & $\mathrm{C}$ & NMBG2136_1658 & Upstream \\
\hline $1767905 *$ & $\mathrm{~T}$ & $\mathrm{~T}$ & $\mathrm{~T}$ & $\mathrm{C}$ & NMBG2136_1658 & Upstream \\
\hline $1768894 *$ & C & $\mathrm{T}$ & $\mathrm{T}$ & $\mathrm{C}$ & $\begin{array}{l}\text { NMBG2136_1658- } \\
\text { NMBG2136_1659 }\end{array}$ & Intergenic \\
\hline $1836562 *$ & $\mathrm{G}$ & $\mathrm{G}$ & $\mathrm{G}$ & $A$ & NMBG2136_1716 & Synonymous \\
\hline $1981679 *$ & CGGGGG & CGGGGG & CGGGGG & CAGCA & $\begin{array}{l}\text { NMBG2136_1863 } \\
\text { (hpuA) }\end{array}$ & $\begin{array}{l}\text { Substitution/ } \\
\text { Frameshift }\end{array}$ \\
\hline 1988383 & TATCC & CATTT & TATCC & CATTT & NMBG2136_1867 & Missense \\
\hline $2005505 *$ & G & $A$ & $A$ & G & $\begin{array}{l}\text { NMBG2136_1879- } \\
\text { NMBG2136_1880 }\end{array}$ & Intergenic \\
\hline 2078354 & ACATT & GCATC & ACATT & ACATT & $\begin{array}{l}\text { NMBG2136_1940- } \\
\text { NMBG2136_1941 }\end{array}$ & Intergenic \\
\hline 2078385 & TAGA & CAGG & TAGA & TAGA & $\begin{array}{l}\text { NMBG2136_1940- } \\
\text { NMBG2136_1941 }\end{array}$ & Intergenic \\
\hline 2078417 & C & $\mathrm{T}$ & C & $\mathrm{T}$ & $\begin{array}{l}\text { NMBG2136_1940- } \\
\text { NMBG2136_1941 }\end{array}$ & Intergenic \\
\hline 2078430 & CA & TG & $\mathrm{CA}$ & TG & $\begin{array}{l}\text { NMBG2136_1940- } \\
\text { NMBG2136_1941 }\end{array}$ & Intergenic \\
\hline
\end{tabular}

Grey shading indicates the isolate has a different sequence from the NMBG2136 reference genome. Variation in hpuA is shown in bold. The 17 variants where NZCM112 diverges from NZ97/052 and NZCM111 are indicated by * in the position column.

HpuA is a phase variable protein that has been shown to play a role in acquiring iron from the host, either from haemoglobin or haptoglobin-haemoglobin complexes (Chen et al., 1998). It has a homopolymeric $\mathrm{G}$ tract, which is prone to slipped-strand mispairing errors, resulting in a subsequent frameshift and premature stop codon (Chen et al., 1998). In this case, the mutation in the NZCM112 isolate did not result from slipped-strand mispairing, although the mutation was in the homopolymeric $\mathrm{G}$ tract. Instead, a CGGGGG sequence from the tract was replaced by a five base pair sequence CAGCA. A ClustalW alignment (Thompson et al., 1994) of the 
nucleotide sequences of NZ97/052 and NZCM112 revealed the location of the mutation in the homopolymeric G tract (Figure 5.1).

To further investigate the role of HpuA in the NZ97/052 household, the predicted translated protein sequences were also aligned using ClustalW (Thompson et al., 1994) (Figure 5.2). This pairwise alignment revealed a premature stop codon very early in the NZCM112 HpuA sequence as a result of the homopolymeric G-tract mutation. From these results, absent or reduced expression of $h p u A$ in NZCM112 isolate would be predicted, relative to hpuA expression in NZ97/052. Expression of $h p u A$ and $h p u B$, which are organised in an operon, have been shown to be linked, with slipped-strand mispairing mutations in hpuA resulting in loss of hpuB translation (Chen et al., 1998). The frameshift and premature stop codon in the hpuA gene would therefore likely result in reduced expression of $\mathrm{HpuB}$ as well. 


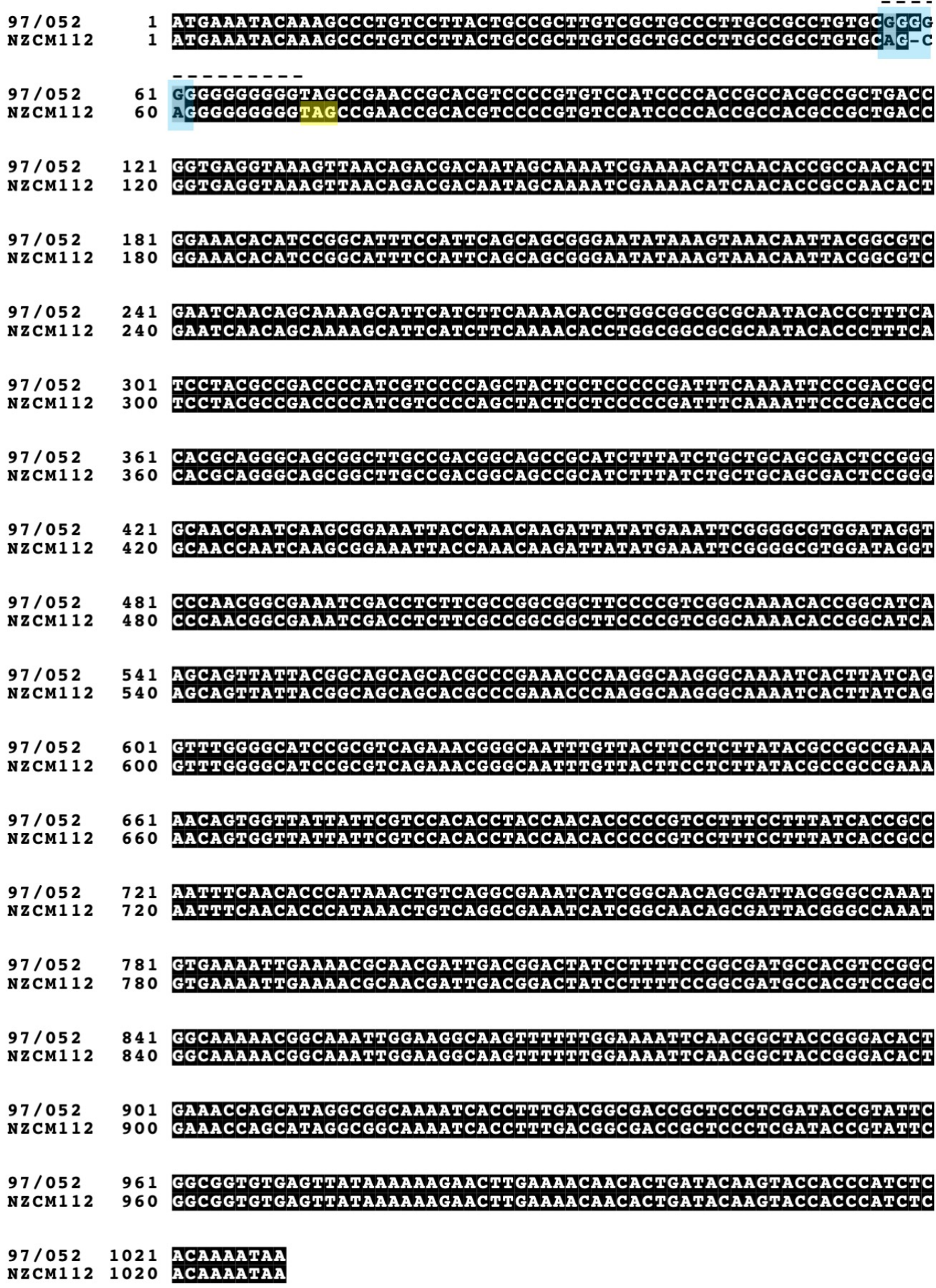

Figure 5.1.Nucleotide sequence alignment of hpuA from NZ97/052 and NZCM112. Aligned using ClustalW (Thompson et al., 1994). Images were created using BOXSHADE 3.21. Black shading indicates conserved residues. Blue box represents the region of mutation in NZCM112. Yellow box indicates the premature stop codon in NZCM112. Dotted line indicates homopolymeric G tract. 


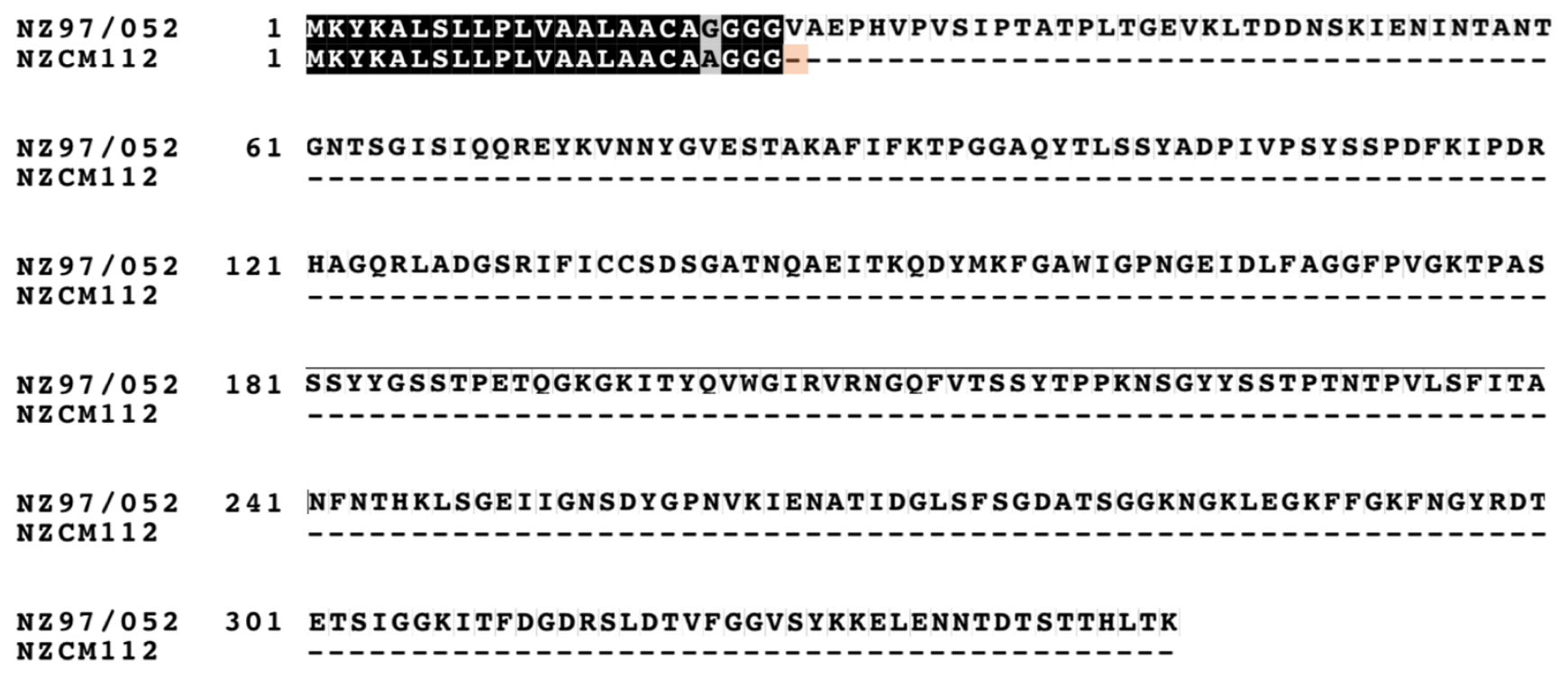

Figure 5.2. Protein sequence alignment of HpuA from NZ97/052 and NZCM112. Aligned using ClustalW

(Thompson et al., 1994). Images were created using BOXSHADE 3.21. Black shading indicates conserved residues and grey shading indicates conservative variation. Orange shading shows the site of the premature stop codon in NZCM112.

\subsubsection{Transcriptome comparison of isolates in the NZ97/052 household}

Many of the SNP variants that differed between NZCM112 compared to NZ97/052 and NZCM111 were in upstream or intergenic regions, suggesting that differences in the ability to inhibit cell migration could be the result of altered gene expression between the isolates. RNAseq analysis was carried out to dissect the genetic differences underpinning the phenotypic variations observed between NZ97/052 and NZCM112. For simplicity, the transcriptome data was limited to comparison of just NZ97/052 and NZCM112 as these two isolates differed in their ability to inhibit cell migration. Total RNA was purified from mid-log-phase N. meningitidis that were co-cultured with 16HBE bronchial epithelial cells for two hours; this was done to detect differences in expression of genes that are regulated in the presence of epithelial cells. Once the RNA was confirmed to be high quality, it was used as a template for the synthesis of double-stranded CDNA, which was shipped to Ambry Genetics (CA, USA) for Illumina 
sequencing. RNA-seq reads were aligned to the ST-8 serogroup B reference genome NMBG2136, using Bowtie2 (Langmead \& Salzberg, 2012). To identify candidate genes, transcriptome comparisons were completed between NZ97/052 and the NZCM112 isolate. The transcription profiles of the NZ97/052 and NZCM112 household isolates in the presence of respiratory epithelial cells differed substantially. A total of 190 transcripts were significantly differentially expressed between NZCM112 and NZ97/052 (adjusted p value < 0.05) (Figure 5.3). Many of the genes that significantly differed in their expression between NZ97/052 and NZCM112 are predicted to play a role in iron homeostasis (indicated by the coloured dots in Figure 5.3).

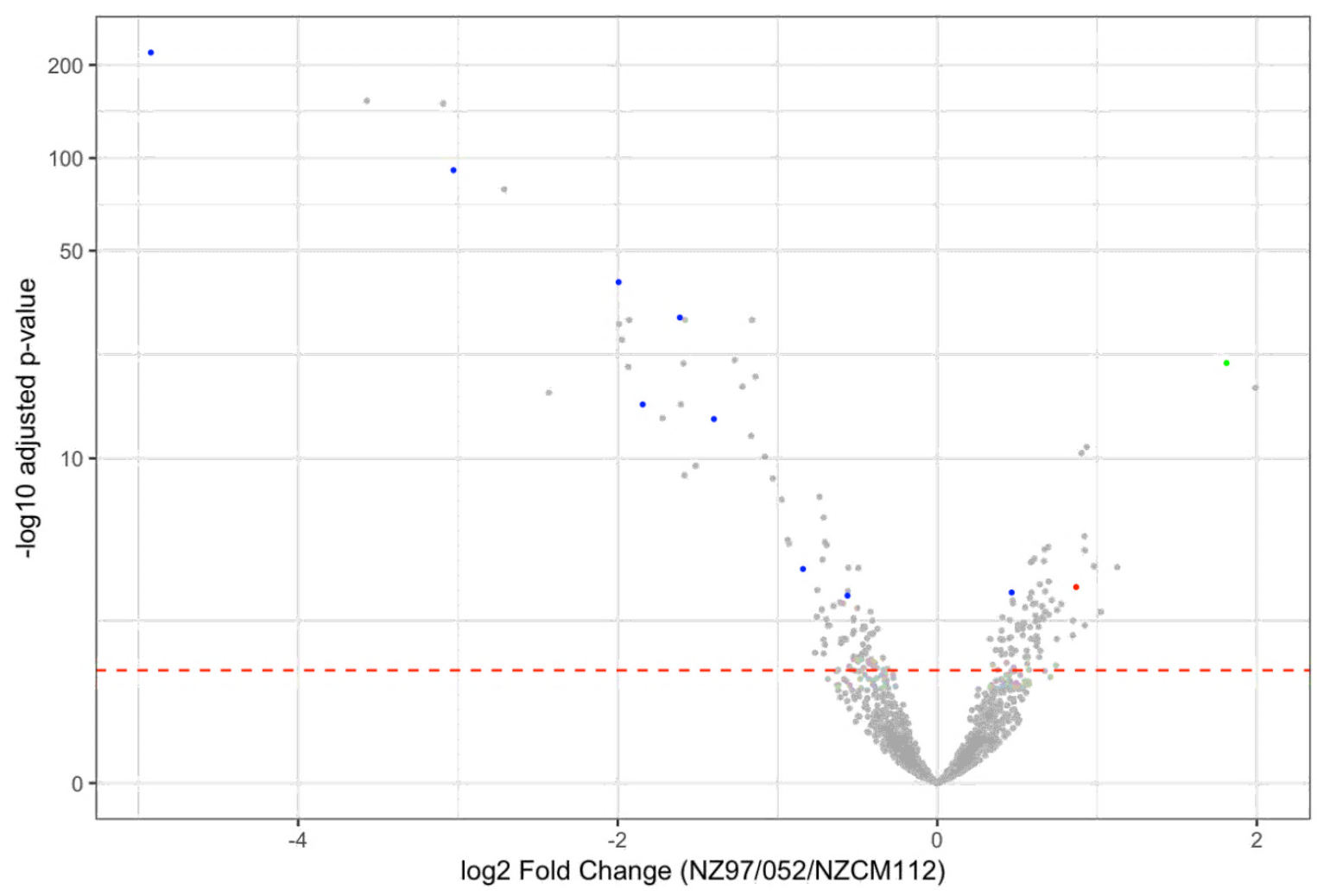

Figure 5.3. Volcano plot of differentially expressed genes between isolates NZ97/052 and NZCM112. Blue dots are genes involved in iron uptake (fetA, fetB, tbpA, tbpB, IbpA, IbpB, efeO, efeU, and NMBG2136_0573). The red dot represents gene hpuA (NMBG2136_1863), the light green dot represents hpuB (NMBG2136_1864). The red line represents the threshold of significance $\left(-\log _{10}\right.$ adjusted $p$-value $\left.>1.30103\right)$. Positive $\log _{2}$ fold change represents higher expression in NZ97/052 (dots on the right side of the volcano plot), while negative $\log _{2}$ fold change represents higher expression in NZCM112 (left side). Analysis was conducted in R (R Core Development Team, 2014) and the figure was produced using package ggplot2 (Wickham, 2009). 
The transcripts with the largest differences in expression between NZ97/052 and NZCM112 were identified using more stringent criteria $\left(\log _{2}\right.$ fold change $+->0.7$, adjusted $p<0.05$ ) and analysed (Figure 5.4), as it was theorised that genes with the largest differences in expression between NZ97/052 and NZCM112 were more likely to have an impact on the phenotype of inhibition of wound repair. Expression levels of these 48 genes are detailed in Appendix Table 9.1. Fourteen proteins had increased expression in NZ97/052 over NZCM112 (Figure 5.4, orange bars). Thirty-four proteins had decreased expression in NZ97/052 relative to NZCM112 (Figure 5.4 , blue bars). The 14 genes with a positive $\log _{2}$ fold change value were genes of interest that may be affecting wound repair inhibition, therefore, these genes were investigated more closely.

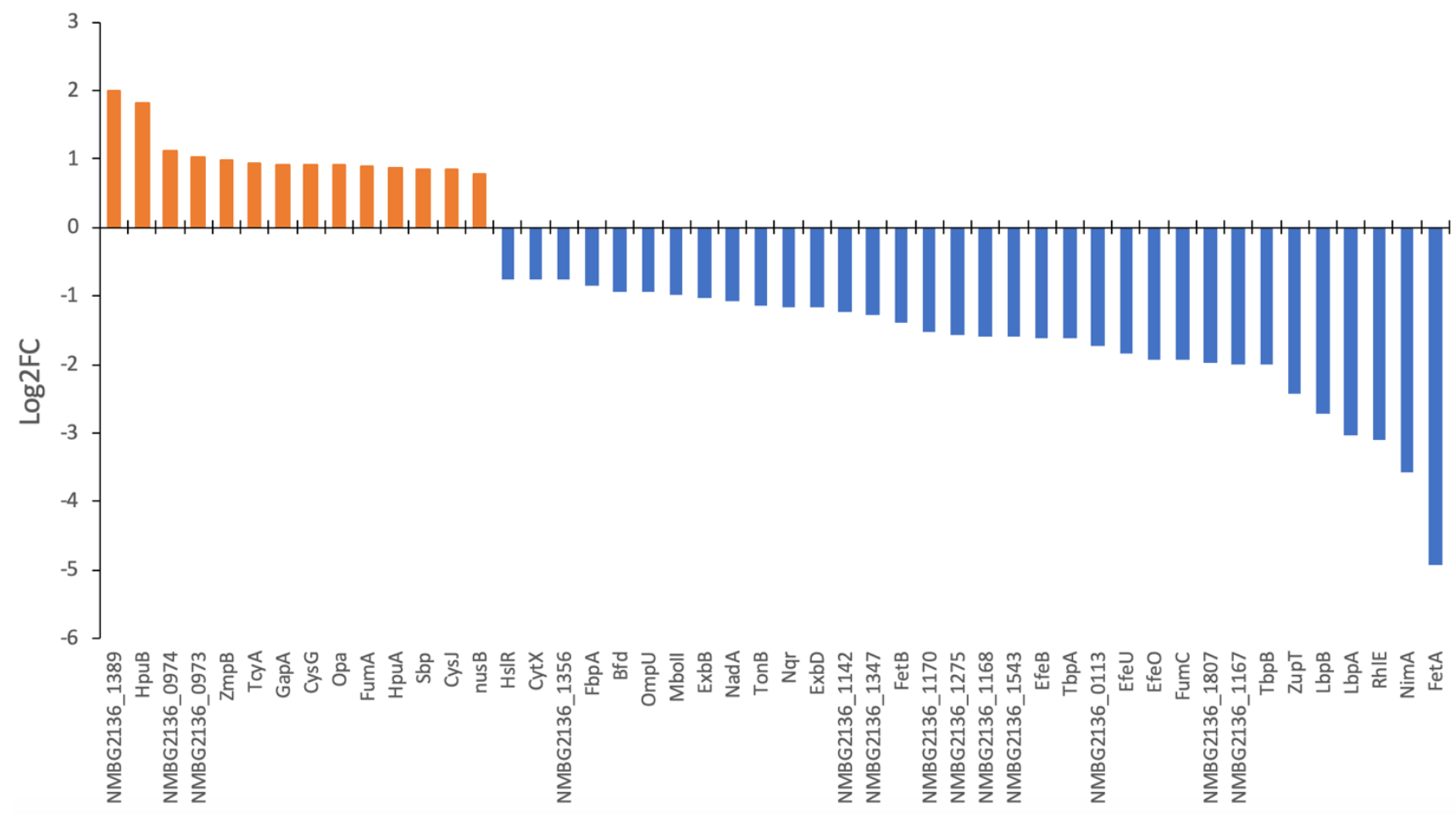

Figure 5.4. Forty-eight of the most highly differentially expressed transcripts between isolates NZ97/052 and NZCM112. $\left(\log _{2} F C+->0.7\right.$, adjusted $\left.p<0.05\right)$. Positive $\log _{2}$ fold change $\left(\log _{2} F C\right)$ values represent increased expression in NZ97/052 (orange bars). Negative $\log _{2} \mathrm{FC}$ values represent increased expression in NZCM112 (blue bars). 
Genomic analysis (Section 5.2.1) revealed a frameshift mutation causing a premature stop codon in hpuA in the NZCM112 isolate (Table 5.2). Transcriptome analysis showed reduced expression of hpuA and $h p u B$ in the NZCM112 isolate, relative to NZ97/052 (Figure 5.4). This result was expected, as these genes are co-transcribed (Chen et al., 1998). The introduction of a premature stop codon likely resulted in an hpuA-hpuB mRNA transcript that is not stabilised by translation or ribosomes, and therefore rapidly degraded. Comparing the chromosomal location of the most highly differentiated transcripts (Table 5.3) to the location of SNP variants did not identify any SNPs, other than that in $h p u A$, that could be predicted to cause a difference in gene expression between NZ97/052 and NZCM112. HpuA was the only protein with altered expression that also had a SNP within the coding region, which differed between NZ97/052 and NZCM112. Another transcript, NMBG2136_1170, is predicted to encode an outer membrane $\beta$ barrel protein. Genomic analysis identified a missense mutation which occurred in both NZ97/052 and NZCM112. However, this transcript was differentially expressed between the two isolates, but as the variant occurred in both isolates the differential expression is not likely due to the nucleotide variant. The differential expression of many transcripts cannot be easily explained by the genomic analysis. However, the loss of HpuA and HpuB in NZCM112 could alter the intracellular environment sufficiently to alter expression of many genes, particularly if it plays an active role in acquisition of iron from the host in the tissue culture model. HpuA has long been known to play a role in the acquisition of iron from haemoglobin, or haemoglobin complexed with haptoglobin. A role for iron acquisition from cultured epithelial cells is supported by the large number of genes involved in acquiring iron that are upregulated in NZCM112, relative to NZ97/052. However, the possibility that other SNPs many indirectly result in altered expression cannot be ruled out.

Table 5.3 lists predicted functional classification of transcripts which differ in expression levels between NZ97/052 and NZCM112. Interestingly, the loss of HpuA and HpuB in NZCM112 appears to result in upregulation of many different iron acquisition genes. These iron acquisition proteins all acquire iron from different sources. For instance, transferrin binding 
proteins TbpA and TbpB are involved in extracting iron from host transferrin in the blood, while LbpA and LbpB are important for extracting iron from lactoferrin, thought to be the main form of iron in the mucosa (Perkins-Balding et al., 2004). Proteins encoded by the $f b p A$, efeO, efeU, and $e f e B$ genes are involved in free iron binding and uptake. Finally, $b f d, f e t A$, and $f e t B$ encode proteins that are involved in pirating iron from siderophores made by other bacteria. This result suggests that NZCM112 is experiencing and responding to low iron levels, likely due to the lack of HpuAB. Additionally, multiple genes encoding proteins that can acquire iron from different host iron sources are upregulated. This implies that, in the cell culture model, the HpuAB uptake system is acquiring iron. This result is strong evidence that expression of HpuA results in iron-replete cells, and isolates that lack HpuA upregulate many other iron-acquisition genes in the bacterial arsenal.

Transcriptome analysis also indicates the sulfur metabolism pathway, including tcyA, cysJ, cys $G$, and sbp genes, is upregulated in the NZ97/052 isolate. A previous study, comparing the transcriptome profiles of disease- and carriage-associated meningococcal isolates, observed upregulation of transcripts involved in sulfur and cystine transport in the disease-associated isolate (Ren et al., 2017). Increased iron may concurrently increase the demand for sulfur in constructing iron-sulfur cluster proteins, or alternatively increased import of sulfur could aid in synthesising the antioxidant glutathione, to protect against reactive species which are generated by iron. Further experiments may reveal the link between increased iron levels and upregulation of the sulfur metabolism pathway. 
Table 5.3. Chromosomal location of the gene, predicted cellular location of the encoded protein, and functional classification of the 48 of the most highly differentially expressed transcripts between isolates NZ97/052 and NZCM112.

\begin{tabular}{|c|c|c|c|c|c|}
\hline Locus Tag & Annotation & $\begin{array}{l}\text { Chromosome } \\
\text { location }\end{array}$ & $\begin{array}{l}\text { Predicted } \\
\text { location }\end{array}$ & $\begin{array}{l}\text { Functional } \\
\text { classification }\end{array}$ & $\log _{2} \mathrm{FC}$ \\
\hline $\begin{array}{l}\text { NMBG2136 } \\
0035\end{array}$ & EfeU & $\begin{array}{l}34740- \\
35579\end{array}$ & $\begin{array}{l}\text { Outer } \\
\text { membrane }\end{array}$ & $\begin{array}{l}\text { Metal iron } \\
\text { binding/ uptake }\end{array}$ & -1.8439282 \\
\hline $\begin{array}{l}\text { NMBG2136_ } \\
0036\end{array}$ & EfeO & $\begin{array}{l}35576- \\
36742\end{array}$ & $\begin{array}{l}\text { Outer } \\
\text { membrane }\end{array}$ & $\begin{array}{l}\text { Metal iron } \\
\text { binding/ uptake }\end{array}$ & -1.9282163 \\
\hline $\begin{array}{l}\text { NMBG2136 } \\
0037\end{array}$ & EfeB & $\begin{array}{l}36886- \\
38151\end{array}$ & $\begin{array}{l}\text { Outer } \\
\text { membrane }\end{array}$ & $\begin{array}{l}\text { Metal iron } \\
\text { binding/ uptake }\end{array}$ & -1.6051488 \\
\hline $\begin{array}{l}\text { NMBG2136 } \\
0113\end{array}$ & $\begin{array}{l}\text { NMBG2136 } \\
0113\end{array}$ & $\begin{array}{l}121363- \\
122058\end{array}$ & Unknown & Unknown & -1.719867 \\
\hline $\begin{array}{l}\text { NMBG2136_ } \\
0171\end{array}$ & ZupT & $\begin{array}{l}163380- \\
164189\end{array}$ & $\begin{array}{l}\text { Outer } \\
\text { membrane }\end{array}$ & $\begin{array}{l}\text { Metal iron } \\
\text { binding/ uptake }\end{array}$ & -2.4308209 \\
\hline $\begin{array}{l}\text { NMBG2136 } \\
0202\end{array}$ & GapA & $\begin{array}{l}199243- \\
200274\end{array}$ & Unknown & Oxidoreductase & 0.92346826 \\
\hline $\begin{array}{l}\text { NMBG2136_ } \\
0421\end{array}$ & $\mathrm{Nqr}$ & $\begin{array}{l}443836- \\
444402\end{array}$ & Cytoplasm & Oxidoreductase & -1.1605483 \\
\hline $\begin{array}{l}\text { NMBG2136_ } \\
0573\end{array}$ & FbpA & $\begin{array}{l}615346- \\
616341\end{array}$ & $\begin{array}{l}\text { Periplasmic } \\
\text { space }\end{array}$ & $\begin{array}{l}\text { Metal iron } \\
\text { binding/ uptake }\end{array}$ & -0.8405905 \\
\hline $\begin{array}{l}\text { NMBG2136 } \\
0631\end{array}$ & NusB & $\begin{array}{l}662800- \\
663225\end{array}$ & Cytoplasm & Transcription & 0.77604229 \\
\hline $\begin{array}{l}\text { NMBG2136_ } \\
0697\end{array}$ & NimA & $\begin{array}{l}728603- \\
729082\end{array}$ & Cytoplasm & Transcription & -3.5706136 \\
\hline $\begin{array}{l}\text { NMBG2136_ } \\
0705\end{array}$ & $\mathrm{Bfd}$ & $\begin{array}{l}734010- \\
734210\end{array}$ & Unknown & Catalytic activity & -0.9289363 \\
\hline $\begin{array}{l}\text { NMBG2136_ } \\
0737\end{array}$ & TсуA & $\begin{array}{l}766930- \\
767757\end{array}$ & $\begin{array}{l}\text { Outer } \\
\text { membrane }\end{array}$ & $\begin{array}{l}\text { Sulfur } \\
\text { metabolism }\end{array}$ & 0.93423459 \\
\hline $\begin{array}{l}\text { NMBG2136_ } \\
0973\end{array}$ & $\begin{array}{l}\text { NMBG2136 } \\
0973\end{array}$ & $\begin{array}{l}983026- \\
984117\end{array}$ & $\begin{array}{l}\text { Plasma } \\
\text { membrane }\end{array}$ & Oxidoreductase & 1.02266001 \\
\hline $\begin{array}{l}\text { NMBG2136 } \\
0974\end{array}$ & $\begin{array}{l}\text { NMBG2136 } \\
0974\end{array}$ & $\begin{array}{l}984252- \\
984935\end{array}$ & Unknown & Oxidoreductase & 1.12628972 \\
\hline $\begin{array}{l}\text { NMBG2136 } \\
0997\end{array}$ & Sbp & $\begin{array}{l}1007334- \\
1008389\end{array}$ & $\begin{array}{l}\text { Outer } \\
\text { membrane }\end{array}$ & $\begin{array}{l}\text { Sulfur } \\
\text { metabolism }\end{array}$ & 0.84916172 \\
\hline $\begin{array}{l}\text { NMBG2136 } \\
1063\end{array}$ & HsIR & $\begin{array}{l}1068276- \\
1068677\end{array}$ & Unknown & $\begin{array}{l}\text { Response to } \\
\text { heat }\end{array}$ & -0.7511499 \\
\hline $\begin{array}{l}\text { NMBG2136 } \\
1076\end{array}$ & CysJ & $\begin{array}{l}1081107- \\
1082921\end{array}$ & Cytoplasm & $\begin{array}{l}\text { Sulfur } \\
\text { metabolism }\end{array}$ & 0.84787993 \\
\hline $\begin{array}{l}\text { NMBG2136 } \\
1080\end{array}$ & CysG & $\begin{array}{l}1085955- \\
1087397\end{array}$ & Cytoplasm & $\begin{array}{l}\text { Sulfur } \\
\text { metabolism }\end{array}$ & 0.92270471 \\
\hline $\begin{array}{l}\text { NMBG2136_ } \\
1142\end{array}$ & $\begin{array}{l}\text { NMBG2136_ } \\
1142\end{array}$ & $\begin{array}{l}1151082- \\
1152551\end{array}$ & $\begin{array}{l}\text { Plasma } \\
\text { membrane }\end{array}$ & Unknown & -1.2203634 \\
\hline $\begin{array}{l}\text { NMBG2136 } \\
1167\end{array}$ & $\begin{array}{l}\text { NMBG2136 } \\
1167\end{array}$ & $\begin{array}{l}1178504- \\
1181443\end{array}$ & Unknown & Unknown & -1.9909041 \\
\hline
\end{tabular}




\begin{tabular}{|c|c|c|c|c|c|}
\hline Locus Tag & Annotation & $\begin{array}{l}\text { Chromosome } \\
\text { location }\end{array}$ & $\begin{array}{l}\text { Predicted } \\
\text { location }\end{array}$ & $\begin{array}{l}\text { Functional } \\
\text { classification }\end{array}$ & $\log _{2} \mathrm{FC}$ \\
\hline $\begin{array}{l}\text { NMBG2136_ } \\
1168\end{array}$ & $\begin{array}{l}\text { NMBG2136 } \\
1168\end{array}$ & $\begin{array}{l}1181456- \\
1182367\end{array}$ & Unknown & Unknown & -1.5827738 \\
\hline $\begin{array}{l}\text { NMBG2136_ } \\
1170\end{array}$ & $\begin{array}{l}\text { NMBG2136 } \\
1170\end{array}$ & $\begin{array}{l}1182970- \\
1184275\end{array}$ & $\begin{array}{l}\text { Outer } \\
\text { membrane }\end{array}$ & Transport & -1.5129915 \\
\hline $\begin{array}{l}\text { NMBG2136_ } \\
1274\end{array}$ & Mboll & $\begin{array}{l}1307396- \\
1309606\end{array}$ & Unknown & Methylation & -0.974063 \\
\hline $\begin{array}{l}\text { NMBG2136_ } \\
1275\end{array}$ & $\begin{array}{l}\text { NMBG2136 } \\
1275\end{array}$ & $\begin{array}{l}1309596- \\
1312370\end{array}$ & $\begin{array}{l}\text { Plasma } \\
\text { membrane }\end{array}$ & Metabolism & -1.5781243 \\
\hline $\begin{array}{l}\text { NMBG2136_ } \\
1276\end{array}$ & RhlE & $\begin{array}{l}1312519- \\
1313679\end{array}$ & Cytoplasm & Transcription & -3.0918234 \\
\hline $\begin{array}{l}\text { NMBG2136 } \\
1347\end{array}$ & $\begin{array}{l}\text { NMBG2136 } \\
1347\end{array}$ & $\begin{array}{l}1404717- \\
1405892\end{array}$ & Cytoplasm & Oxidoreductase & -1.267371 \\
\hline $\begin{array}{l}\text { NMBG2136_ } \\
1351\end{array}$ & FumC & $\begin{array}{l}1410800- \\
1412188\end{array}$ & Cytoplasm & Metabolism & -1.9338733 \\
\hline $\begin{array}{l}\text { NMBG2136_ } \\
1356\end{array}$ & $\begin{array}{l}\text { NMBG2136 } \\
1356\end{array}$ & $\begin{array}{l}1417032- \\
1417190\end{array}$ & Unknown & Catalytic activity & -0.7649251 \\
\hline $\begin{array}{l}\text { NMBG2136 } \\
1358\end{array}$ & Opa & $\begin{array}{l}1419339- \\
1420010\end{array}$ & $\begin{array}{l}\text { Outer } \\
\text { membrane }\end{array}$ & Adhesion & 0.91991125 \\
\hline $\begin{array}{l}\text { NMBG2136 } \\
1389\end{array}$ & $\begin{array}{l}\text { NMBG2136 } \\
1389\end{array}$ & $\begin{array}{l}1460046- \\
1461464\end{array}$ & $\begin{array}{l}\text { Plasma } \\
\text { membrane }\end{array}$ & Catalytic activity & 1.99110613 \\
\hline $\begin{array}{l}\text { NMBG2136_ } \\
1421\end{array}$ & LbpA & $\begin{array}{l}1497406- \\
1500240\end{array}$ & $\begin{array}{l}\text { Outer } \\
\text { membrane }\end{array}$ & $\begin{array}{l}\text { Metal iron } \\
\text { binding/ uptake }\end{array}$ & -3.0279845 \\
\hline $\begin{array}{l}\text { NMBG2136 } \\
1422\end{array}$ & LbpB & $\begin{array}{l}1500237- \\
1502471\end{array}$ & $\begin{array}{l}\text { Outer } \\
\text { membrane }\end{array}$ & $\begin{array}{l}\text { Metal iron } \\
\text { binding/ uptake }\end{array}$ & -2.711906 \\
\hline $\begin{array}{l}\text { NMBG2136 } \\
1491\end{array}$ & FumA & $\begin{array}{l}1573559- \\
1575082\end{array}$ & Cytoplasm & Metabolism & 0.90218988 \\
\hline $\begin{array}{l}\text { NMBG2136 } \\
1543\end{array}$ & $\begin{array}{l}\text { NMBG2136 } \\
1543\end{array}$ & $\begin{array}{l}1633542- \\
1635904\end{array}$ & Unknown & Unknown & -1.5887339 \\
\hline $\begin{array}{l}\text { NMBG2136 } \\
1600\end{array}$ & ExbD & $\begin{array}{l}1703238- \\
1703672\end{array}$ & $\begin{array}{l}\text { Plasma } \\
\text { membrane }\end{array}$ & Transport & -1.1648299 \\
\hline $\begin{array}{l}\text { NMBG2136 } \\
1601\end{array}$ & ExbB & $\begin{array}{l}1703675- \\
1704337\end{array}$ & $\begin{array}{l}\text { Plasma } \\
\text { membrane }\end{array}$ & Transport & -1.029428 \\
\hline $\begin{array}{l}\text { NMBG2136_ } \\
1602\end{array}$ & TonB & $\begin{array}{l}1704403- \\
1705245 \\
\end{array}$ & $\begin{array}{l}\text { Plasma } \\
\text { membrane }\end{array}$ & $\begin{array}{l}\text { Metal iron } \\
\text { binding/ uptake }\end{array}$ & -1.1383945 \\
\hline $\begin{array}{l}\text { NMBG2136_ } \\
1639\end{array}$ & TbpA & $\begin{array}{l}1741456- \\
1744203 \\
\end{array}$ & $\begin{array}{l}\text { Outer } \\
\text { membrane }\end{array}$ & $\begin{array}{l}\text { Metal iron } \\
\text { binding/ uptake }\end{array}$ & -1.6113297 \\
\hline $\begin{array}{l}\text { NMBG2136_ } \\
1640\end{array}$ & TbpB & $\begin{array}{l}1744290- \\
1746434\end{array}$ & $\begin{array}{l}\text { Outer } \\
\text { membrane }\end{array}$ & $\begin{array}{l}\text { Metal iron } \\
\text { binding/ uptake }\end{array}$ & -1.9947031 \\
\hline $\begin{array}{l}\text { NMBG2136 } \\
1708\end{array}$ & NadA & $\begin{array}{l}1824974- \\
1826086\end{array}$ & $\begin{array}{l}\text { Outer } \\
\text { membrane }\end{array}$ & Adhesion & -1.0795069 \\
\hline $\begin{array}{l}\text { NMBG2136 } \\
1785\end{array}$ & OmpU & $\begin{array}{l}1901222- \\
1902634\end{array}$ & $\begin{array}{l}\text { Outer } \\
\text { membrane }\end{array}$ & Transport & -0.9356677 \\
\hline $\begin{array}{l}\text { NMBG2136 } \\
1807\end{array}$ & $\begin{array}{l}\text { NMBG2136 } \\
1807\end{array}$ & $\begin{array}{l}1922387- \\
1924498\end{array}$ & Unknown & Unknown & -1.9737996 \\
\hline
\end{tabular}




\begin{tabular}{|c|c|c|c|c|c|}
\hline Locus Tag & Annotation & $\begin{array}{l}\text { Chromosome } \\
\text { location }\end{array}$ & $\begin{array}{l}\text { Predicted } \\
\text { location }\end{array}$ & $\begin{array}{l}\text { Functional } \\
\text { classification }\end{array}$ & $\log _{2} F C$ \\
\hline $\begin{array}{l}\text { NMBG2136 } \\
1863\end{array}$ & HрuA & $\begin{array}{l}1981624- \\
1982652\end{array}$ & $\begin{array}{l}\text { Outer } \\
\text { membrane }\end{array}$ & $\begin{array}{l}\text { Metal iron } \\
\text { binding/ uptake }\end{array}$ & 0.86872091 \\
\hline $\begin{array}{l}\text { NMBG2136_ } \\
1864\end{array}$ & HpuB & $\begin{array}{l}1982683- \\
1985115\end{array}$ & $\begin{array}{l}\text { Outer } \\
\text { membrane }\end{array}$ & $\begin{array}{l}\text { Metal iron } \\
\text { binding/ uptake }\end{array}$ & 1.81016584 \\
\hline $\begin{array}{l}\text { NMBG2136_ } \\
1880\end{array}$ & FetA & $\begin{array}{l}2007474- \\
2009654\end{array}$ & $\begin{array}{l}\text { Outer } \\
\text { membrane }\end{array}$ & $\begin{array}{l}\text { Metal iron } \\
\text { binding/ uptake }\end{array}$ & -4.9220283 \\
\hline $\begin{array}{l}\text { NMBG2136_ } \\
1881\end{array}$ & FetB & $\begin{array}{l}2010353- \\
2011318\end{array}$ & $\begin{array}{l}\text { Outer } \\
\text { membrane }\end{array}$ & $\begin{array}{l}\text { Metal iron } \\
\text { binding/ uptake }\end{array}$ & -1.3980111 \\
\hline $\begin{array}{l}\text { NMBG2136_ } \\
1968\end{array}$ & CytX & $\begin{array}{l}2109884- \\
2111107\end{array}$ & $\begin{array}{l}\text { Plasma } \\
\text { membrane }\end{array}$ & Transport & -0.7541613 \\
\hline $\begin{array}{l}\text { NMBG2136_ } \\
1994\end{array}$ & $\mathrm{ZmpB}$ & $\begin{array}{l}2131585- \\
2132235\end{array}$ & $\begin{array}{l}\text { Plasma } \\
\text { membrane }\end{array}$ & Metabolism & 0.979251 \\
\hline
\end{tabular}

Positive $\log _{2} \mathrm{FC}$ values represent increased expression in NZ97/052 (dark grey). Negative $\log _{2} \mathrm{FC}$ values represent increased expression in NZCM112 (light grey). Cellular location and functional classification were determined using the UniProt database (UniProt, 2008).

Genes which were upregulated in NZ97/052 were examined further to identify additional candidates predicted to alter wound repair inhibition. The cellular location and functional classification were predicted for each of the fourteen transcripts more highly expressed in NZ97/052, using the UniProt database (UniProt, 2008). Functional analysis of these proteins is summarised in Figure 5.5A. As previously noted, four transcripts encoded proteins predicted to be involved in cystine and sulfur uptake or metabolism (cysG, cysJ, tcyA and sbp). Three transcripts encoded proteins predicted to have oxidoreductase activity (gapA , NMBG2136_0974 and NMBG2136_0973), two encoded proteins involved in metal ion binding and uptake (hpuA and $h p u B$ ), and two encoded proteins predicted to have metabolic activity (fumA and $z m p B$ ). Other transcripts were predicted to encode proteins that function in transcription (nusB), catalytic activity (NMBG2136_1389), and cell adhesion (opa). The predicted cellular locations were also analysed (Figure 5.5B). Five transcripts were predicted to encode outer membrane proteins ( $s b p, o p a, h p u A, h p u B$, and tcyA). These genes were of particular interest because outer membrane proteins are more likely to interact with host cells, and could be responsible for the inhibition of cell migration. Three transcripts expressed at higher levels in NZ97/052 encoded putative cytoplasmic proteins and three were predicted to be in the plasma membrane. The cellular location of three of these proteins was unknown. 
Transcripts which had a - $\log _{2}$ fold change $<-0.7$ had greater expression in isolate NZCM112 relative to NZ97/052, which represents more than a 1.6 fold difference in transcript levels. Functional analysis was predicted for 34 genes highly upregulated in NZCM112 relative to NZ97/052 (Figure 5.5C). Cellular location and molecular function were unknown for five transcripts missing from the UniProt database, as they were not predicted to encode functional proteins (NMBG2136_1168, NMBG2136_1543, NMBG2136_1807, NMBG2136_1167, NMBG2136_0113). The largest proportion of upregulated genes were involved in metal ion binding and uptake, with 13 of the 34 transcripts predicted to encode proteins with this function ( $f b p A, b f d$, ton $B, f e t B, e f e B, t b p A, t b p B$, efeU, efeO, zupT, IbpA, IbpB, and fet $A$ ). Five proteins were predicted to have transport activity $(c y t X, o m p U, \operatorname{exbB}, \operatorname{exbD}$, and NMBG2136_1170), three were predicted as metabolic proteins (NMBG2136_1275, fumC, and $\operatorname{nimA}$ ), and two were predicted with oxidoreductase activity (nqr and NMBG2136_1347). Other transcript products were predicted to function in cell adhesion ( $\operatorname{nad} A)$, catalytic activity (NMBG2136_1356), DNA methylation (mboll), transcription ( $r h / E)$, and response to heat (hs/R). Uniprot analysis of the cellular location (Figure 5.5D) predicted 11 transcripts to encode outer membrane proteins, nine with an unknown location, seven were predicted as plasma membrane proteins, six were predicted as cytoplasmic proteins, and one was predicted in the periplasmic space. 
A

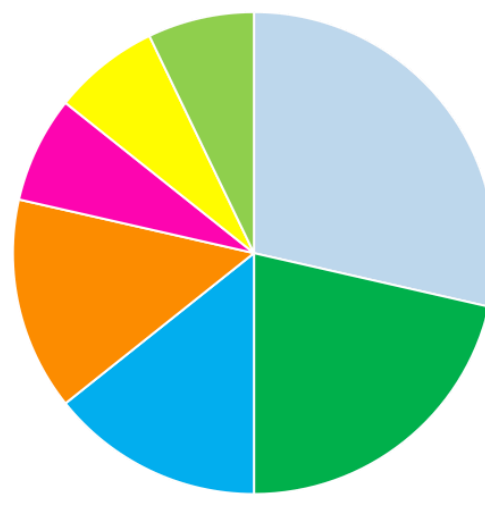

= Sulfur uptake and meta bolism (4)

- Oxidoreductase activity (3)

- Metal ion binding/uptake (2)

- Metabolism (2)

- Cell adhesion (1)

- Catalytic activity (1)

- Transcription (1)

C

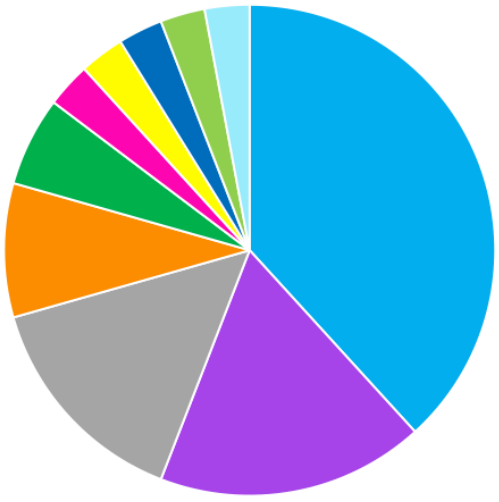

B

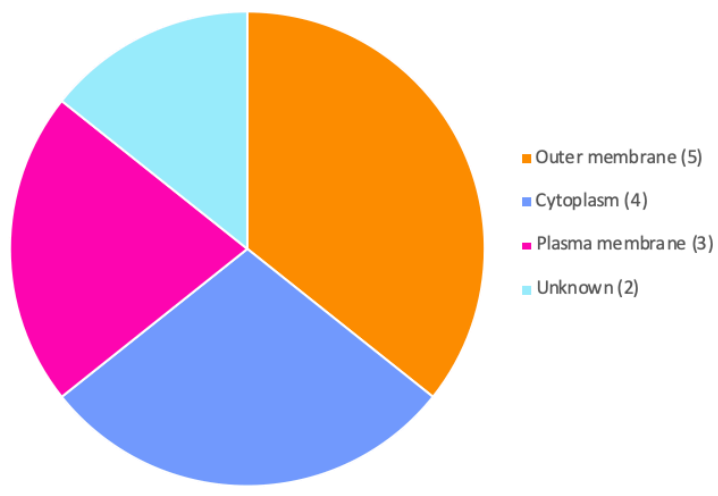

D

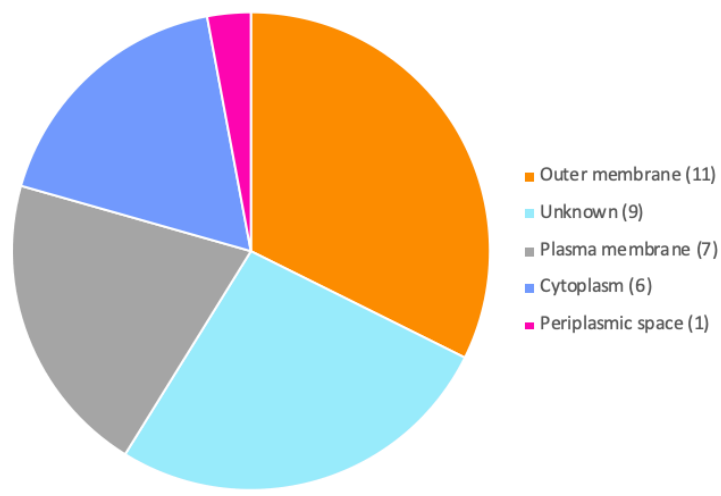

Figure 5.5. Predicted cellular location and functional classification of differentially expressed genes according to the UniProt database. (A) Functional analysis of genes upregulated in NZ97/052 predicted 3/14 encode proteins involved in oxidoreductase activity, and 4/14 encode proteins involved in sulfur uptake and metabolism. (B) Cellular location analysis of transcripts upregulated in NZ97/052 show 5/14 were predicted to encode outer membrane proteins (sbp, opa, hpuA, hpuB, and tcyA). (C) Functional analysis of genes upregulated in NZCM112 relative to NZ97/052 predict 13/34 transcripts were involved in metal iron binding/uptake. (D) Cellular location of 9/34 transcript products upregulated in NZCM112 were unknown, they were either absent from the UniProt database or their function was unknown (see Table 5.3 for details).

\subsubsection{Candidate genes selected for deletion from NZ97/052}

The 48 genes which had the largest change in expression between NZ97/052 and NZCM112 were examined to identify any candidate genes for deletion. Genes which were previously deleted from disease-associated isolates at ESR, and were tested for any impact on wound repair, were eliminated (Appendix, Section 9.2). The functions of the remaining genes were 
hypothesised from the available literature to determine whether any were likely to play a role in wound repair inhibition. The gene expression analysis suggested a striking difference in iron uptake between NZ97/052 and NZCM112. Therefore, there was a focus on genes involved in iron acquisition or regulation. Genome and transcriptome results comparing carriage- and disease-associated isolates highlighted fet $A$, fur, $h p u A$, and $h p u B$ as top candidate genes which could affect host cell wound repair inhibition. The rationale for selecting each gene is described below. The well-characterised adhesin, opa, was of interest as this outer membrane protein was more highly expressed in NZ97/052 compared to NZCM112; however, the opa gene could not be deleted as there are three copies of this gene in the NMBG2136 genome.

\subsubsection{1 fetA}

Siderophores are small but very powerful iron chelators, which are often produced by colonising bacteria to obtain iron from the host (Raymond et al., 2003). Although iron is abundant within the host, most of it is sequestered and unavailable to colonising bacteria. Siderophores bind free iron with high affinity, enabling them to scavenge iron. One example of a high-affinity siderophore is enterobactin, produced by enteric bacteria to acquire iron (Raymond et al., 2003). No potential N. meningitidis siderophores have been identified (Archibald \& DeVoe, 1980). However, some studies have suggested $N$. meningitidis may be able to utilise siderophores which have been secreted by other bacteria (Rutz et al., 1991). FetA is an outer membrane protein and enterobactin receptor expressed by some meningococcal isolates (Thompson et al., 2003). FetA was previously named FrbP (Beucher \& Sparling, 1995), and it encodes a TonB-dependent transporter. The gene was renamed in 1999 when Carson et al (1999) showed low affinity binding and transport of ferric enterobactin by FetA (Carson et al., 1999; Pettersson et al., 1995).

The transcriptome data identified differential expression of the fet $A$ gene between diseaseassociate isolate NZ97/052 and NZCM112, with higher levels of fetA expression in NZCM112. It was selected for deletion as it was the most differentially expressed gene between the two isolates. The partner FetA transporter is FetB, and fetB was also differentially expressed 
between NZ97/052 and NZCM112. These genes are presumed to be co-transcribed (Carson et al., 1999), therefore, deletion of fetA will also likely lead to loss of expression of fetB. FetA is a major antigen and is known to be expressed on the surface of $N$. meningitidis (Thompson et al., 2003). Therefore, the possibility that FetA could be interacting with host cells, and thereby interfering with normal wound repair, was investigated.

\subsubsection{2 fur}

Iron can be toxic to bacteria at high concentrations due to its catalytic activity and capacity to generate reactive molecules via the Fenton reaction (Andrews et al., 2003; Hood \& Skaar, 2012). In the host, free iron is limiting and colonising pathogens must employ various mechanisms to obtain sufficient iron for growth. Transition metal levels must therefore be carefully controlled by the bacteria in order to prevent cellular damage (Guilhen et al., 2013). The Ferric Uptake Regulator (Fur) is present in many bacterial genera and is a key regulator of multiple genes that maintain bacterial iron homeostasis (Troxell \& Hassan, 2013). Fur binds iron as a co-repressor, blocking transcription of specific genes when iron levels are sufficient (Bagg \& Neilands, 1987; Delany et al., 2003). Up to $50 \%$ of iron-regulated genes in N. meningitidis contain a putative Fur binding sequence in their promoter regions (Grifantini et al., 2003). When free iron levels are low, which is the usual state inside the human host, Fur undergoes a conformational change which prevents binding to DNA and it no longer functions as a repressor. However, Grifantini et al demonstrated that Fur can also act as a transcriptional activator; their study described Furregulated genes which were upregulated with the addition of iron (2003). This is particularly interesting as results reported in Chapter Four (Figure 4.2) and transcriptome data, described in Section 5.2.2, implicates iron-regulated genes in the difference in wound repair inhibition by disease-associated and carriage associated isolates. The carriage-associated isolate, NZCM112, appeared to express a factor which increased wound repair inhibition in the presence of iron (Figure 4.2). It is possible this could be an iron-activated Fur-regulated gene. Although fur was not differentially regulated between the isolates, it was selected for deletion as it is involved in iron regulated expression of many $N$. meningitidis genes. 


\subsubsection{3 hрuAB}

Most iron in the body is stored within haemoglobin (Andrews, 1999). Haptoglobin is an acute phase protein, primarily produced in the liver (Shih et al., 2014). Haptoglobin can bind haemoglobin to form haptoglobin-haemoglobin complexes, which prevents scavenging microbes from utilising haemoglobin and limits oxidative damage of haemoglobin to specific organs (Lim et al., 1998; Tseng et al., 2004). In N. meningitidis, hpuA is a phase variable gene that encodes the first of a pair of outer membrane proteins; HpuA and HpuB. HpuAB binds haemoglobin, haptoglobin, and haptoglobin-haemoglobin complexes, and allows $N$. meningitidis to thrive in the low-iron environment (Chen et al., 1998; Lewis \& Dyer, 1995; Rohde \& Dyer, 2004). HpuA is a lipoprotein, anchored to the outer membrane, and is largely surface exposed (Lewis et al., 1997). HpuB is predicted to be $\beta$-barrel structure, forming a pore in the outer membrane (Rohde \& Dyer, 2004) (Figure 5.6). The hpuB gene does not contain its own promoter; rather, the $h p u A$ and $h p u B$ genes are co-transcribed, and deletion or frameshift mutations of hpuA result in lack of expression of $h p u B$ (Chen et al., 1998; Lewis et al., 1997). HpuB was more highly expressed in NZ97/052 compared to NZCM112, and multiple bases were substituted in hpuA in NZCM112, resulting in a frameshift mutation and premature stop codon. HpuA and HpuB are two of the five outer membrane proteins which were upregulated in $\mathrm{NZ97/052,} \mathrm{therefore} \mathrm{these} \mathrm{genes} \mathrm{were} \mathrm{identified} \mathrm{as} \mathrm{top} \mathrm{candidates.} \mathrm{Allelic} \mathrm{replacement}$ deletions of the $h p u A$ and $h p u B$ genes were created individually, as well as both $h p u A$ and $h p u B$ together. Presumably the replacement of the hpuA gene with an antibiotic resistance cassette had a polar effect, resulting in loss of $h p u B$ expression as well. 


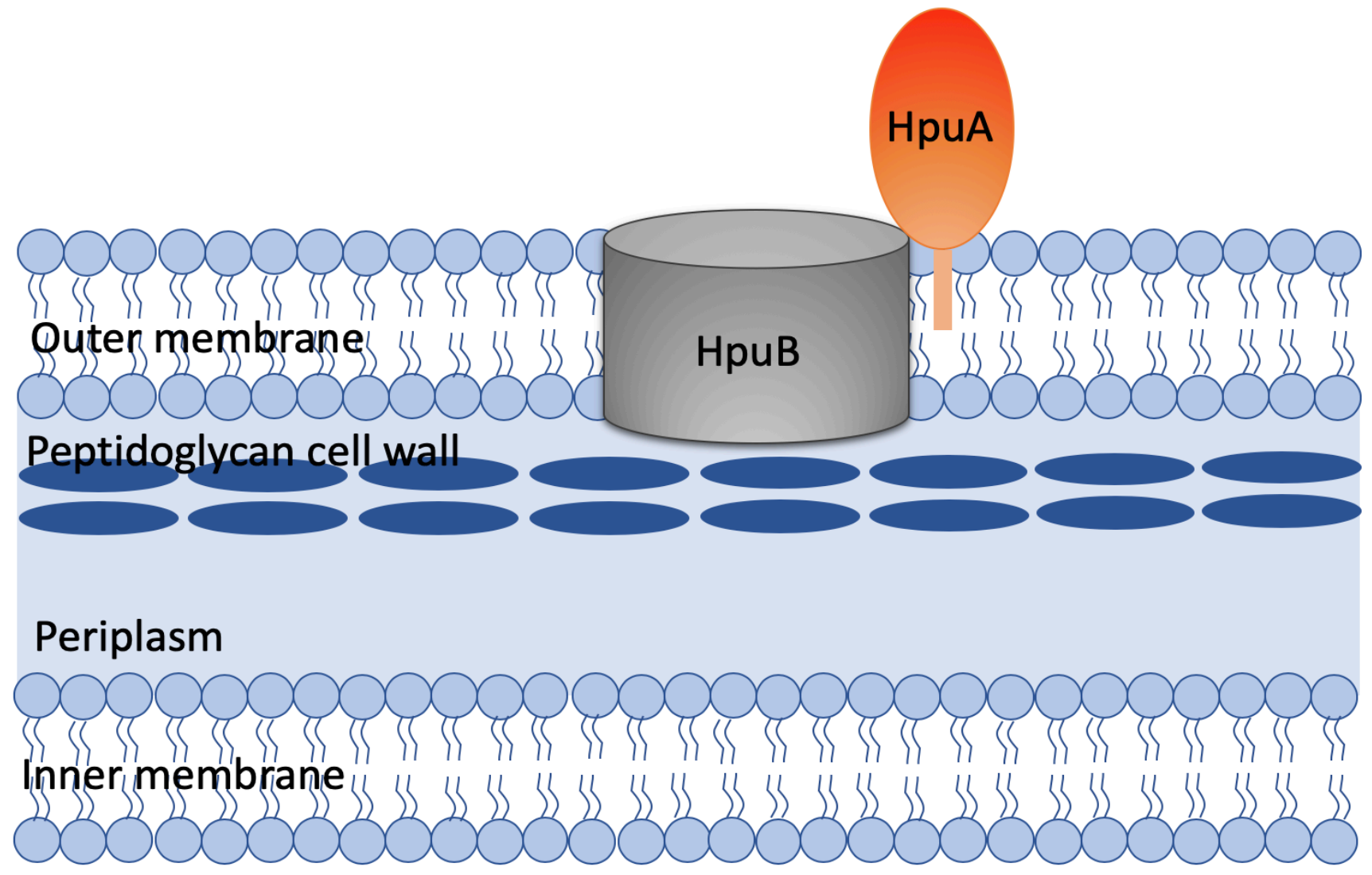

Figure 5.6. Schematic representation of HpuAB iron acquisition system in $N$. meningitidis.

\subsubsection{Neisseria meningitidis mutagenesis}

Candidate genes were deleted from parent strain NZ97/052. NZ97/052 was selected because it inhibits host cell wound repair and loss of this function was screened for. The goal was to revoke the ability of a disease-associated isolate to inhibit wound repair, making it resemble a carriagelike isolate. Doing so would indicate successful identification of the meningococcal gene responsible for cell migration inhibition.

$N$. meningitidis is a naturally competent bacterium, and is competent in all phases of growth (Kline et al., 2003). Natural competence for transformation aids in the generation of gene deletion mutants. Transformation involves the binding and uptake of naked DNA by the bacterium and homologous recombination (Ambur et al., 2012). For mutagenesis of targeted genes the wild-type allele is replaced with a selectable antibiotic cassette. The mutagenic DNA 
transformed into N. meningitidis is a fusion product comprised of two roughly $200 \mathrm{bp}$ sequences from either side of the targeted gene, flanking the gene which confers resistance to kanamycin, aphA3. The recombinant mutagenic DNA was made by PCR fusion (Figure 5.7). The amplified sequences flanking the targeted gene were fused to the aphA3 gene, amplified by PCR from a plasmid. The three fragments were joined together by PCR, using the outermost primers, and with internal priming of complementary sequences enabling the fusion of all three products. The resulting fusion product was amplified and verified, then used for natural transformation. Transformation of the mutagenic PCR product resulted in double homologous recombination, which occurred in the two flanking regions. This resulted in allelic replacement, with the gene of interest replaced by the aphA3 antibiotic resistance cassette. Mutants were then selected on plates supplemented with kanamycin. In Neisseria, DNA uptake sequences (DUS) are short, conserved sequences dispersed throughout the bacterial genome, which allow N. meningitidis to favour uptake of DNA from Neisseriae over foreign DNA, which amplifies transformation efficiency (Frye et al., 2013; Spencer-Smith et al., 2016). The aphA3 primers used in this study were designed to contain the 10 bp 5'-GCCGTCTGAA-3' DUS, to facilitate transformation. Because the mutagenic DNA is a linear PCR product, it cannot replicate and the transformed strain only becomes resistant to kanamycin following integration of the DNA into the chromosome. Mutagenesis primers were designed to ensure that the majority of the targeted gene was removed, with minimal polar effects on adjacent genes. 

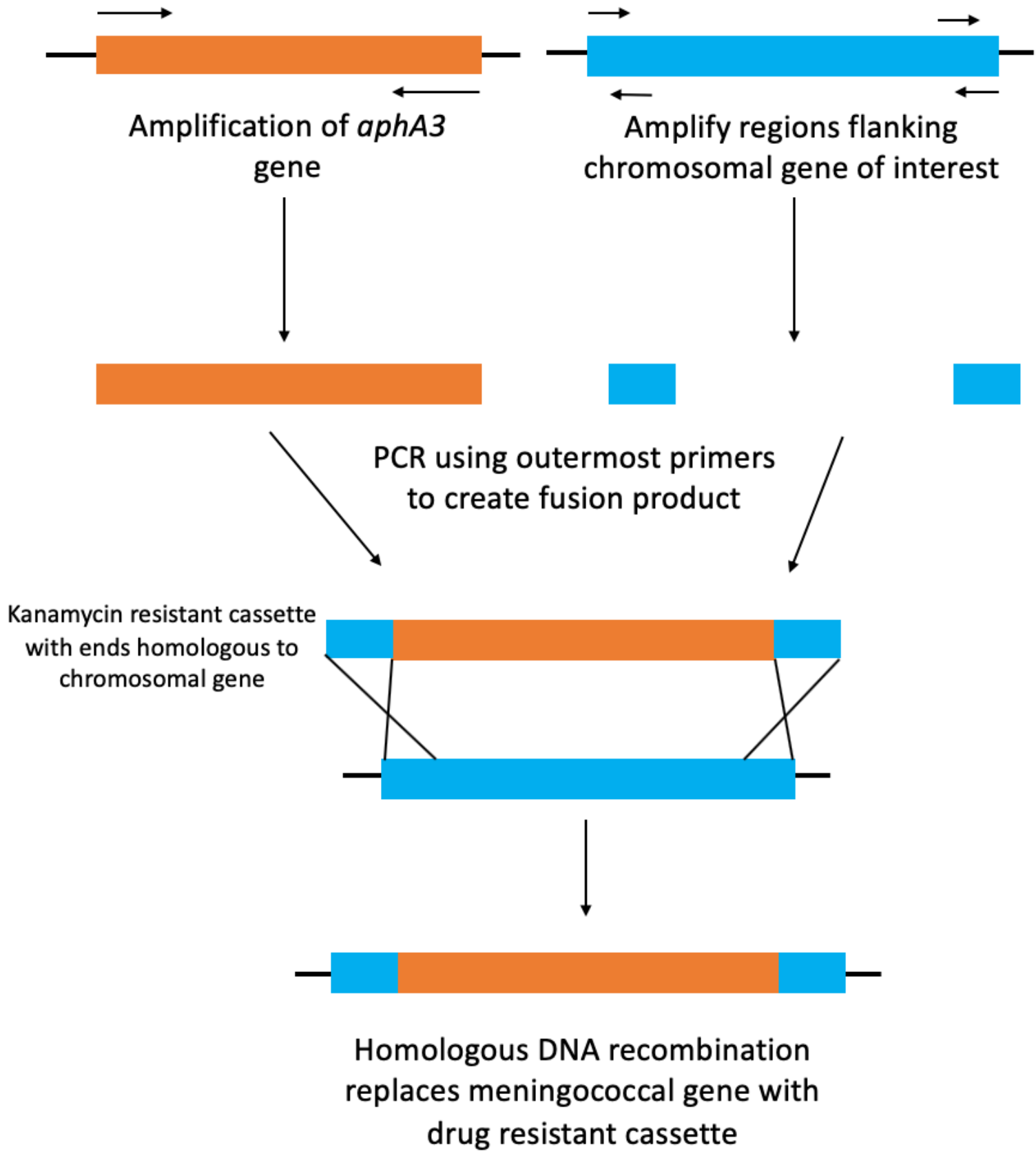

Figure 5.7. Overview of gene replacement with antibiotic resistant cassette in Neisseria meningitidis. The orange bars represent the aphA3 gene, which is amplified from a plasmid. The blue bars represent chromosomal DNA from NZ97/052. 


\subsubsection{PCR amplification of regions of homology flanking candidate genes}

Four genes of interest were replaced in the wild-type isolate with an antibiotic resistant cassette, flanked by regions homologous to the targeted gene. One double mutant was also constructed. Once primers were designed to amplify the flanking regions of the target genes, fusion PCR products were generated, with the aphA3 gene positioned between flanking regions. The reactions to amplify the sequences flanking the candidate genes were carried out using NZ97/052 genomic DNA as a template. Specific primers used are detailed in Chapter Two, Table 2.4. Primers were designed, where necessary, to anneal to the aphA3 primers to enable internal priming. To ensure sufficient crossover, the region of homology between primers was at least 20 bp. PCR products were run on an agarose gel (Figure 5.8A). The predicted product sizes for PCR targets were determined based on the $N$. meningitidis NMBG2136 reference genome. The expected product sizes were $217 \mathrm{bp}$ for the region upstream of fet $A$ and $177 \mathrm{bp}$ for the region downstream. The expected product size for the regions flanking the fur gene were $189 \mathrm{bp}$ upstream and 179 bp downstream. The expected PCR product sizes for the hpuA gene were 195 bp for the upstream region and $152 \mathrm{bp}$ for the downstream region. The expected product size for the region downstream of $h p u B$ was $233 \mathrm{bp}$. The actual PCR product sizes for all amplified gene regions were as expected and were used for generation of the PCR fusion products. As seen in Figure $5.8 \mathrm{~A}$, initially only the region downstream of the $h p u B$ gene was successfully amplified. New primers to amplify the upstream region of $h p u B$ were designed and used for a second PCR. The expected product size was $190 \mathrm{bp}$, and the actual product size was as expected (Figure 5.8B). The size of all PCR products corresponded to the expected size, therefore these products were used to proceed with generating mutagenic PCR fusion products for fet $A$, fur, hpuA, and hpuB. 


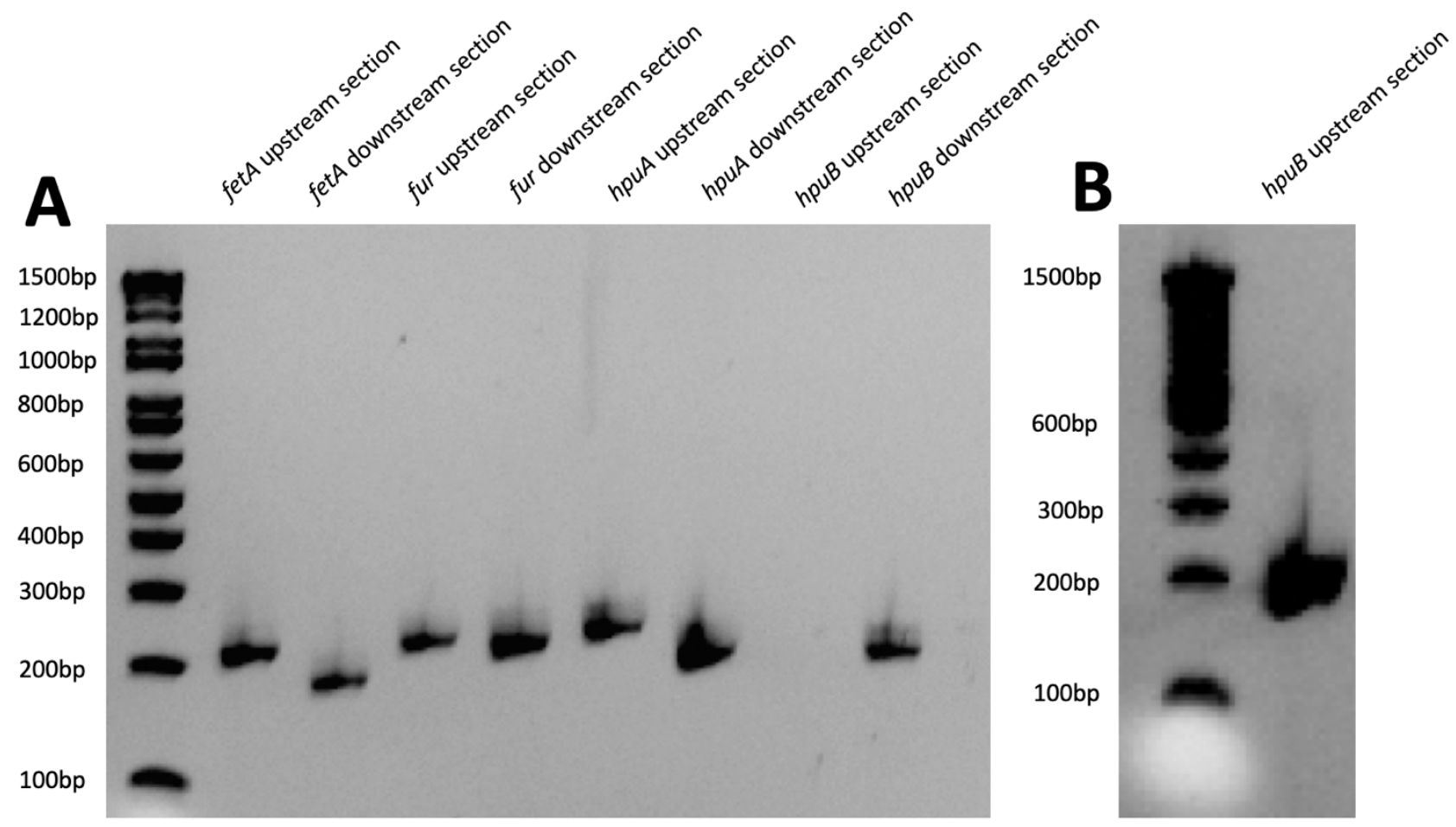

Figure 5.8. Amplification of DNA flanking candidate genes, visualised on $2 \%$ agarose gel. (A) Amplification of $f e t A$, fur, hpuA upstream and downstream regions of candidate genes, and downstream region of hpuB. Expected PCR product sizes were 217 and 177 bp (fetA), 189 and 179 bp (fur), and 195 and 152 (hpuA). The downstream section of $h p u B$ was predicted to be $233 \mathrm{bp}$. (B) Repeat amplification of region upstream to hpuB gene with new primers, expected product size 190 bp. 100 bp O'RangeRuler DNA ladder (Thermo Fisher Scientific, USA) was used to estimate PCR product size.

\subsubsection{PCR amplification of aphA3 gene}

The pUC19 Km (kanamycin) plasmid DNA was used as a template for PCR amplification of the aphA3 gene using primers listed in Chapter Two, Table 2.4. The expected PCR product size was $\sim 1300 \mathrm{bp}$. The DNA gel revealed the PCR product was the size expected (Figure 5.9), so was purified and used in subsequent steps to generate the PCR fusion products. 


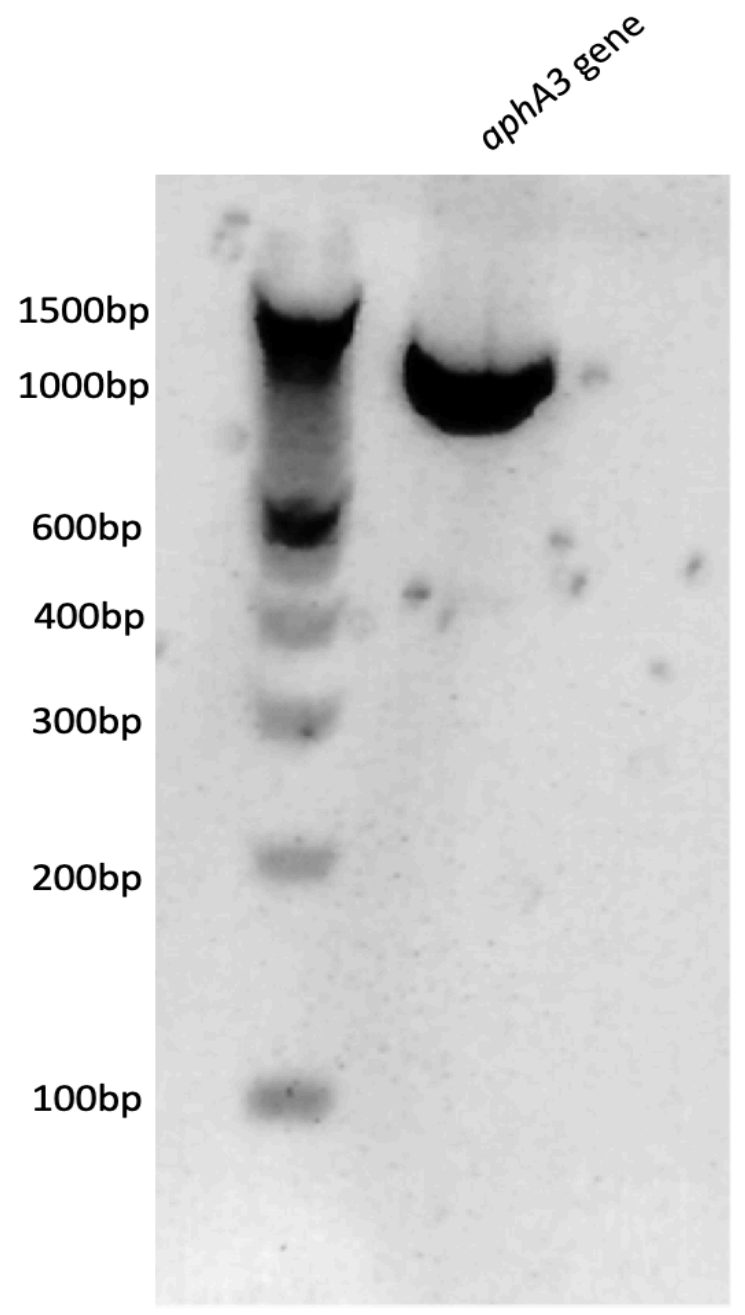

Figure 5.9. PCR of aphA3 gene, amplified from pUC19 Km, visualized on $2 \%$ agarose gel. The $100 \mathrm{bp}$ O'RangeRuler DNA ladder (Thermo Fisher Scientific, USA) was used to estimate PCR product size.

\subsubsection{Construction of fusion products and mutagenesis}

For each candidate gene, the purified PCR products of the sequences flanking the gene and the purified aphA3 PCR product were combined and used as a template in a final PCR to form a fusion product. Only the outermost primers were used, as the internal primers had been designed to enable internal priming between the PCR products. The expected size of the fusion products were approximately $1700 \mathrm{bp}$ for each candidate gene, which was in line with the size of the products seen on the agarose gel (Figure 5.10). To generate the hpuA-hpuB double deletion, a PCR fusion product was made with the upstream flanking region from $h p u A$, the 
aphA3 gene, and the downstream flanking region from hpuB. Double homologous recombination in sites upstream of $h p u A$ and downstream of $h p u B$ would result in the replacement of both hpuA and hpuB with aphA3.

For each fusion product, multiple rounds of PCR were carried out and their products combined to obtain a sufficiently large quantity of DNA for mutagenesis of NZ97/052, as previous gene deletions completed in the laboratory found that higher concentrations of DNA resulted in more colonies. Purified PCR products were used for natural transformation of NZ97/052 as described in Chapter Two, Section 2.5. The next day, six individual colonies per mutagenesis were selected to verify natural transformation and allelic exchange.

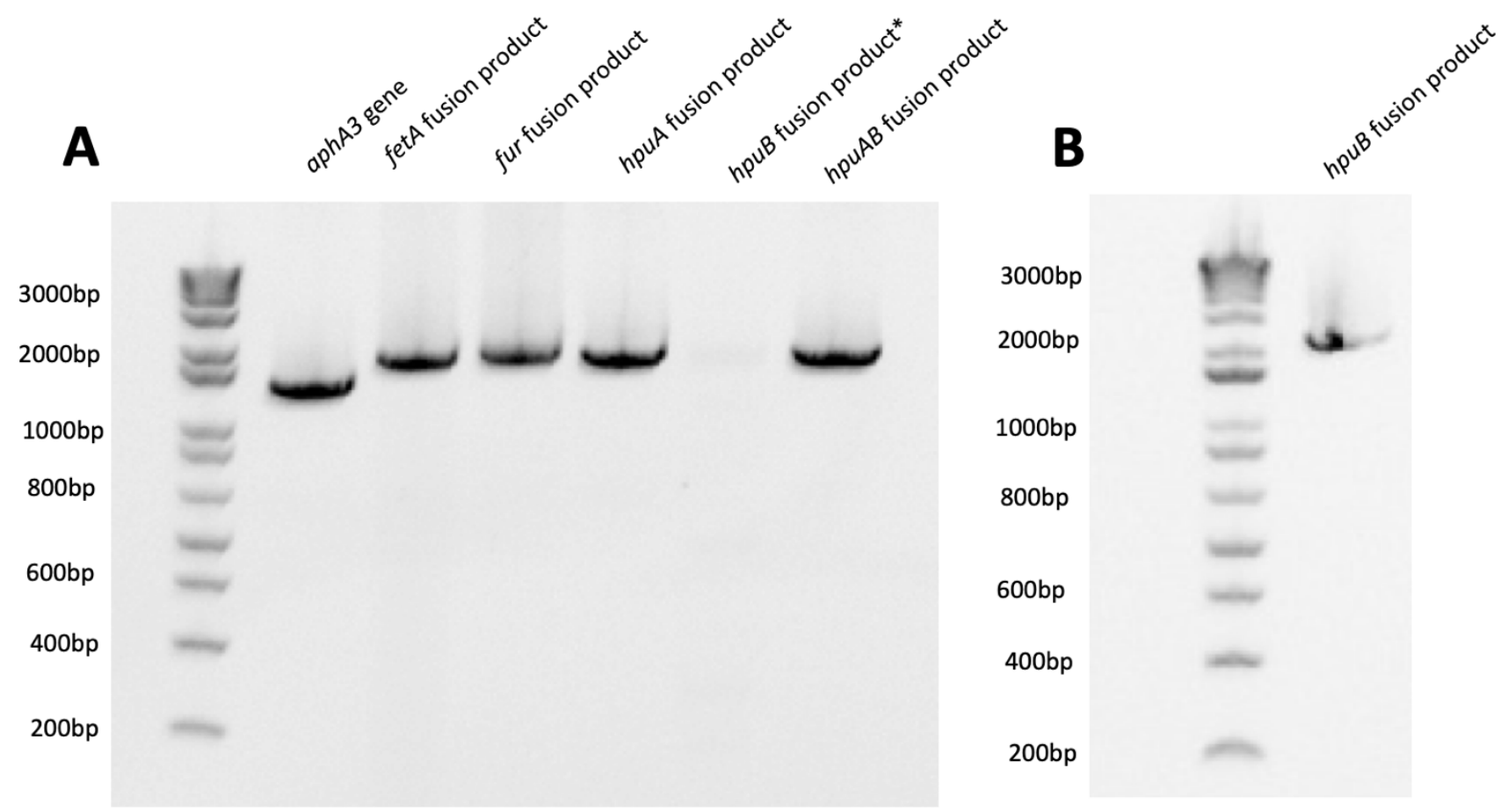

Figure 5.10. PCR to form recombinant gene fusion products visualised on a $2 \%$ agarose gel. Amplification of apha3, fetA, fur, hpuA, and hpuAB fusion products. Expected product sizes were approximately $1700 \mathrm{bp}$. (B) Repeat amplification of $h p u B$ fusion product. The expected product size was approximately $1700 \mathrm{bp} .200 \mathrm{bp}$ O'RangeRuler DNA ladder (Thermo Fisher Scientific, USA) was used to estimate PCR product size. *hpuB fusion product resulted in a weak band on agarose gel. 


\subsubsection{Selection and validation of gene deletion mutants}

All of the putative mutants were selected by the ability to grow in the presence of kanamycin. Normally the N. meningitidis NZ97/052 isolate lacks kanamycin resistance and is unable to grow on agar containing kanamycin. Kanamycin resistance indicates insertion of the aphA3 gene into the chromosome. Insertion of aphA3 into the correct site on the chromosome was confirmed by carrying out PCR on kanamycin-resistant colonies.

Genomic DNA was purified from the NZ97/052 wild-type and each of the mutants and was used as a template for PCR, using the outermost relevant primers. In every case the predicted size of the product would be different between the wild-type and the mutant, as the introduced aphA3 gene differed in size from the deleted coding regions. PCR product sizes from the mutants were compared to those from the wild-type parent by agarose gel electrophoresis. Figure 5.11 demonstrates the substantial difference between the size of the gene region isolated from the wild-type NZ97/052 isolate and NZ97/052 $\triangle h p u A$, indicating successful allelic replacement of the target gene with aphA3.

To confirm that the aphA3 gene fully replaced the target gene, the amplified gene regions were sequenced, ensuring that the junction between aphA3 and the chromosome was where predicted (Appendix, Section 9.3). Target gene regions were amplified by PCR, using the outermost primers, purified, and then sequenced with the same primers by in-house Sanger sequencing at ESR. The sequence was analysed by 4Peaks (Griekspoor \& Groothuis, 2005) and NCBI BLAST (Altschul et al., 1990) to compare the sequence to that of the reference genome and identify the junction with the aphA3 kanamycin resistance gene. Examining the sequence confirmed that in all of the transformants the main coding regions of the target genes fet $A$, fur, hpuA, hpuAB, and $h p u B$ were replaced with aphA3. These gene deletion mutants were subsequently tested in cell migration assays. 


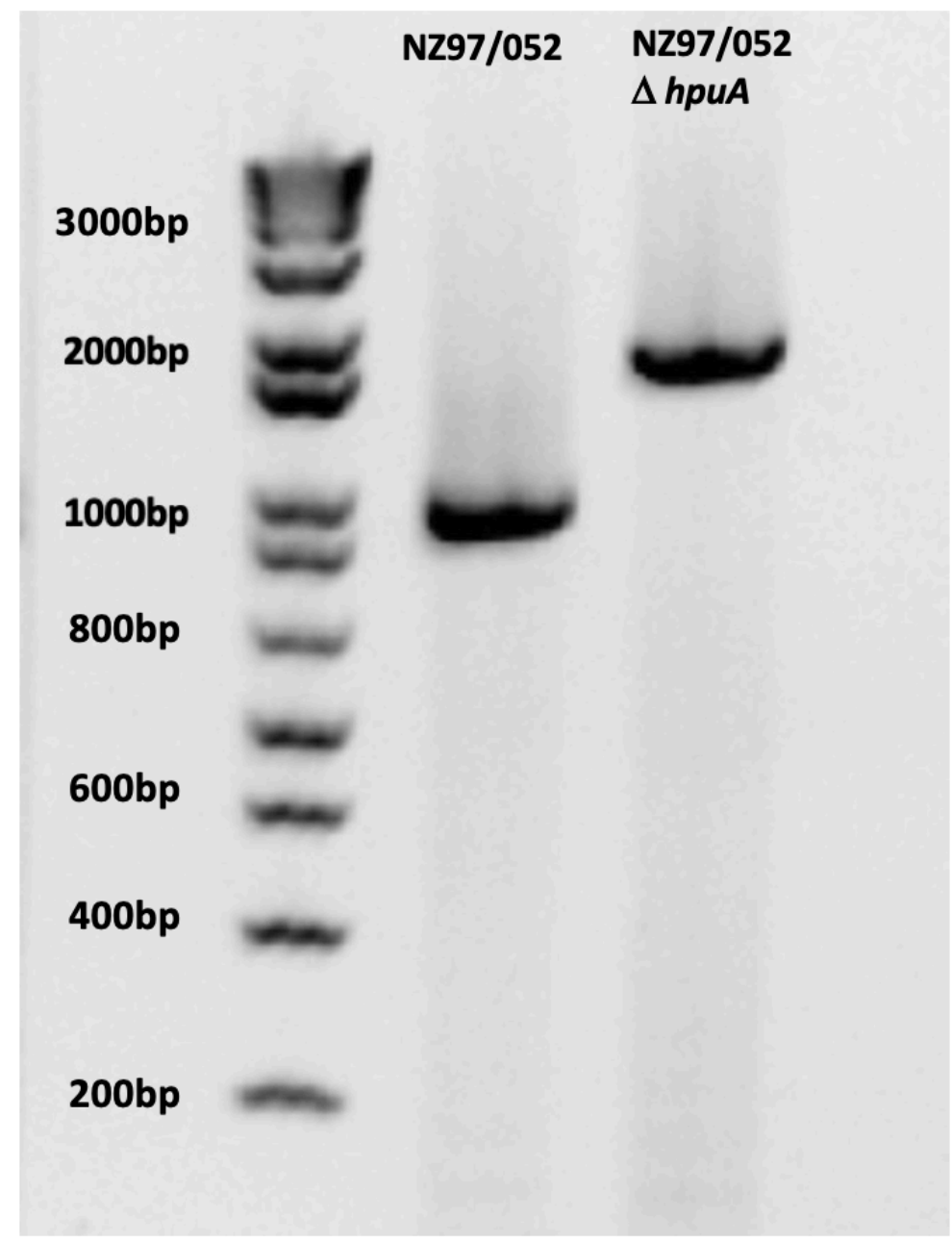

Figure 5.11. PCR to confirm gene deletion of hpuA gene in NZ97/052 isolate, visualised on a $2 \%$ agarose gel. 200bp O'RangeRuler DNA ladder (Thermo Fisher Scientific, USA) was used to estimate PCR product size.

\subsubsection{Effect of FetA and Fur on wound repair}

An Oris cell migration assay measures the extent of host cell migration after the infection with bacteria. N. meningitidis isolates NZ97/052, NZCM112, NZ97/052 $\Delta$ fetA, and NZ97/052 $\Delta$ fur were used to infect $16 \mathrm{HBE}$ cells in the 96 -well Oris plate. A control without added bacteria was also included. No significant difference in wound repair inhibition was seen comparing the $\mathrm{NZ97/052} \mathrm{isolate} \mathrm{to} \mathrm{either} \mathrm{of} \mathrm{the} \mathrm{mutants,} \mathrm{NZ97/052} \Delta$ fetA or NZ97/052 $\Delta$ fur (Figure 5.12). These results suggest that meningococcal genes fet $A$ and fur do not play a role in inhibition of wound repair of respiratory epithelial cells. It was not entirely unexpected that fet $A \operatorname{did}$ not 
impact wound repair inhibition, as this gene had higher expression in NZCM112. However, it could be interesting to delete this gene from the NZCM112 isolate to examine if this gene had an impact on any phenotypes, such as bacterial growth rate, or the increased ability to inhibit wound repair during iron supplementation.

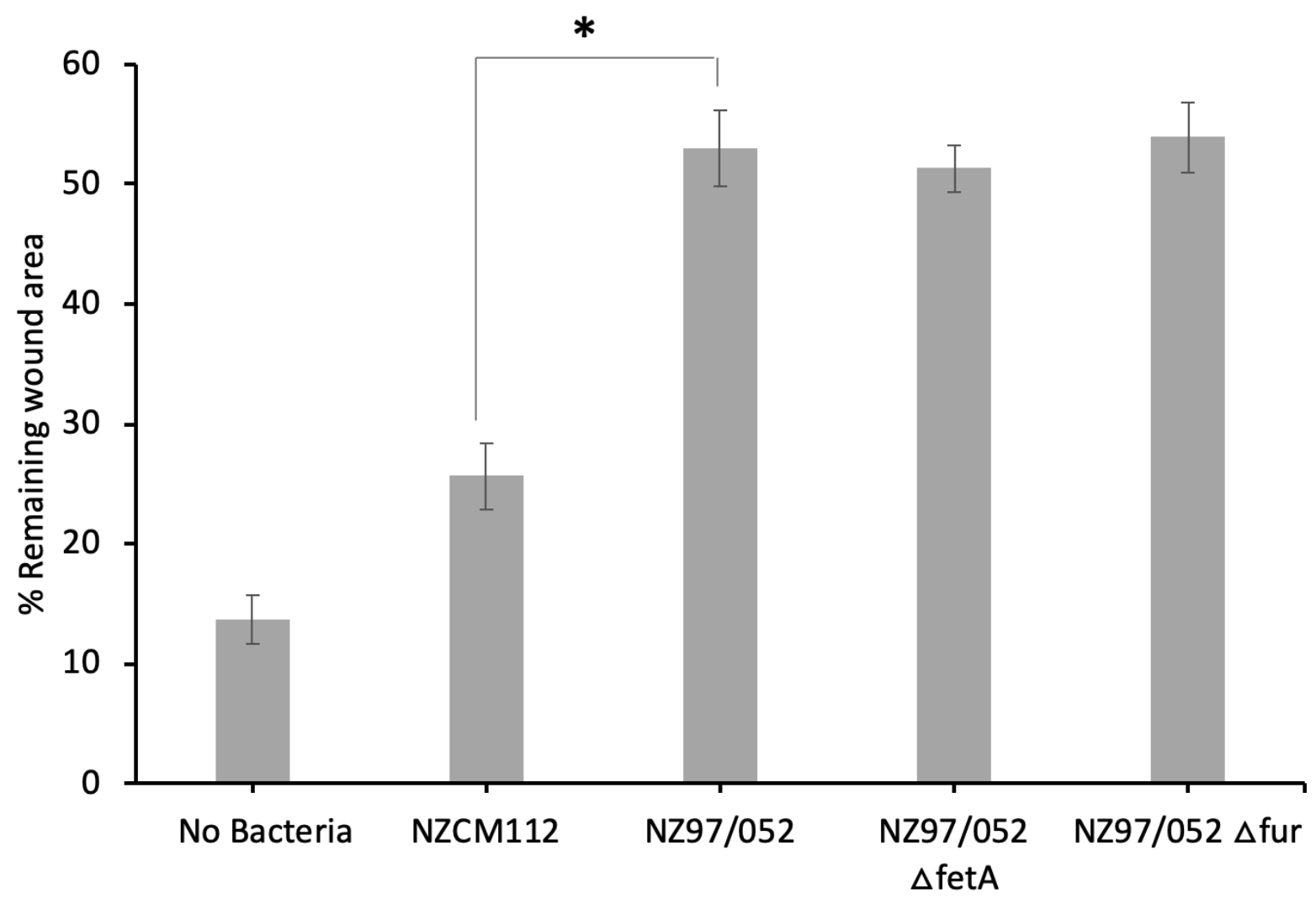

Figure 5.12. Deletion of $f e t A$ and fur from NZ97/052 does not impact cell migration inhibition. Error bars show standard deviation. Data points are determined from means of eight replicates. Significant difference between NZ97/052 and NZCM112 was determined with One-Way ANOVA. P-value $<0.05=*$.

\subsubsection{Effect of HpuA and HpuB on wound repair}

Both genome and transcriptome data identified $h p u A$ and $h p u B$ as the top candidate meningococcal genes that may have an impact on inhibition of host cell migration. The hpuA gene in NZCM112 was the only gene in this household group identified by genome sequencing that had a mutation predicted to result in loss of function. However, it is known that loss of 
hpuA expression results in loss of $h p u B$ expression, and the transcriptome data confirmed that this is the case in NZCM112. Since both genes are located on the bacterial cell surface or in the outer membrane, either could be playing a role in inhibition of wound repair. These genes were deleted from wild-type NZ97/052 isolate, both separately and together. Because $h p u A$ and $h p u B$ coding regions form an operon, the $h p u A$ deletion mutant likely also does not express $h p u B$. Therefore, a $h p u B$ deletion strain was also constructed and tested. Because $h p u B$ is downstream in the operon, it was assumed that hpuA would still be expressed. N. meningitidis isolates NZ97/052, NZCM112, NZ97/052 $\Delta h p u A$, NZ97/052 $\triangle h p u B$, and NZ97/052 $\Delta h p u A B$ (the double deletion) were used to infect $16 \mathrm{HBE}$ cells in a 96-well Oris plate. A control without added bacteria was also included. The allelic replacement of the hpuA gene, or both $h p u A$ and $h p u B$, completely nullified the ability for NZ97/052 isolates to inhibit wound repair (Figure 5.13). Bronchial epithelial cells infected with the parent NZ97/052 isolate had $53.35 \%$ of the initial wound area after incubation for 16 hours. Deletion of both $h p u A$ and $h p u A B$ from NZ97/052 both caused a significant reduction in wound repair inhibition compared to the wild-type NZ97/052 isolate $(p<0.05)$, where $21.94 \%$ and $23.41 \%$ of the wound area remained, respectively. Reduction in inhibition of cell migration was so striking that the area remaining for uninfected bronchial epithelial cells did not differ significantly from cells infected with the NZ97/052 $\triangle$ hpuA strain after 16 hours $(p=0.875)$. However, deletion of only hpuB from NZ97/052 did not have a significant effect on wound repair $(p=0.781)$. These exciting results show a novel role for meningococcal gene hpuA in host cell migration inhibition and identify HpuA as the meningococcal factor responsible for wound repair inhibition by the NZ97/052 household isolates.

These results also indicate $h p u A$ and $h p u B$ mutants have a different phenotype for cell migration inhibition of respiratory epithelial cells. An analysis of HpuA and HpuB binding to haemoglobin and haptoglobin-haemoglobin using flow cytometry was conducted by Rohde et al (2004). An hpuA deletion reduced affinity of binding to these complexes, although HpuB expressed alone was sufficient to bind to haemoglobin, haptoglobin, and haptoglobinhaemoglobin. Because $h p u A$ and $h p u B$ are co-transcribed, with hpuA upstream of $h p u B$ (Chen et 
al., 1998; Lewis et al., 1997), deletion of $h p u A$ in NZ97/052 likely abrogates expression of $h p u B$, while the $h p u B$ mutant likely still expresses hpuA. Experiments conducted by Rhode et al (2004), suggest that both the $h p u A$ and $h p u B$ mutants generated in this study will lower the affinity of binding to haemoglobin and haptoglobin-haemoglobin. Results from the cell migration assay (Figure 5.13) demonstrate that deletion of $h p u B$ does not impact wound repair inhibition, a striking finding that suggests that the inhibition of wound repair inhibition may not be due to binding of haemoglobin, haptoglobin, or haptoglobin-haemoglobin complexes.

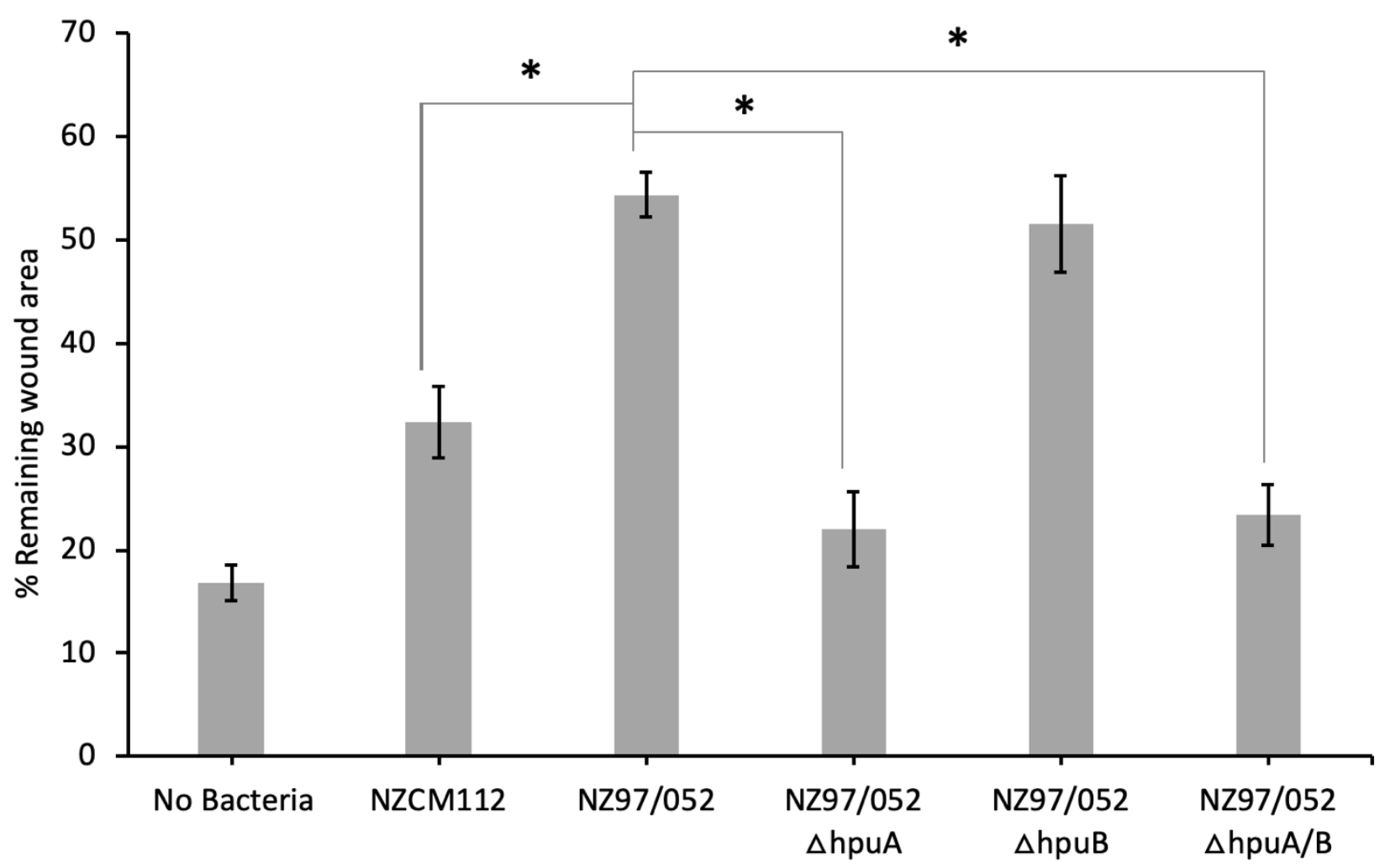

Figure 5.13. Deletion of $h p u A$ and the double mutant $h p u A B$ from NZ97/052 significantly reduces cell migration inhibition, but deletion of hpuB alone does not. Error bars show standard deviation. Data points are determined from means of 12 replicates. The significant difference between NZ97/052 and other isolates was determined with One-Way ANOVA. P-value $<0.05=*$. 
It was investigated whether the phenotype of NZCM112, which has a frameshift mutation in hpuA, was replicated in the NZ97/052 $\triangle$ hpuA mutant. Specifically, experiments tested if iron sulfate supplementation of the hpuA mutant would result in an increase in the ability of this isolate to inhibit cell migration, as it did for NZCM112 (Figure 4.2). Epithelial cell wound repair assays were completed with $20 \mu \mathrm{M}$ iron sulfate $\left(\mathrm{FeSO}_{4}\right)$ supplementation (Figure 5.14). The addition of $\mathrm{FeSO}_{4}$ had no effect on the migration of bronchial cells alone or bronchial cells infected with NZ97/052. As expected, supplementation with iron sulfate caused a significant increase in wound repair inhibition by NZCM112 ( $<<0.001)$. Surprisingly, there was a similar significant effect of iron on the NZ97/052 $\triangle h p u A$ deletion mutant (Figure 5.14). The addition of $20 \mu \mathrm{M} \mathrm{FeSO}_{4}$ caused a significant increase in wound repair inhibition by NZ97/052 $\Delta h p u A$, with $20.37 \%$ of initial wound area remaining without added iron and $47.85 \%$ of the initial wound area with added iron $(p<0.001)$. These results were surprising and suggest there is likely a second meningococcal factor, other than HpuA, which plays a role in cell migration inhibition. Expression of the second factor may be induced by high free ferrous iron levels, a phenomenon that was seen in both carriage isolates and the $\triangle h p u A$ mutant. The presence of HpuA may mask the effect of the second factor, since addition of iron does not alter wound repair inhibition in the HpuA-expressing strain NZ97/052, although it is important to note that the status of the hpuA gene is not known in the other carriage isolates tested in Figure 4.3. 


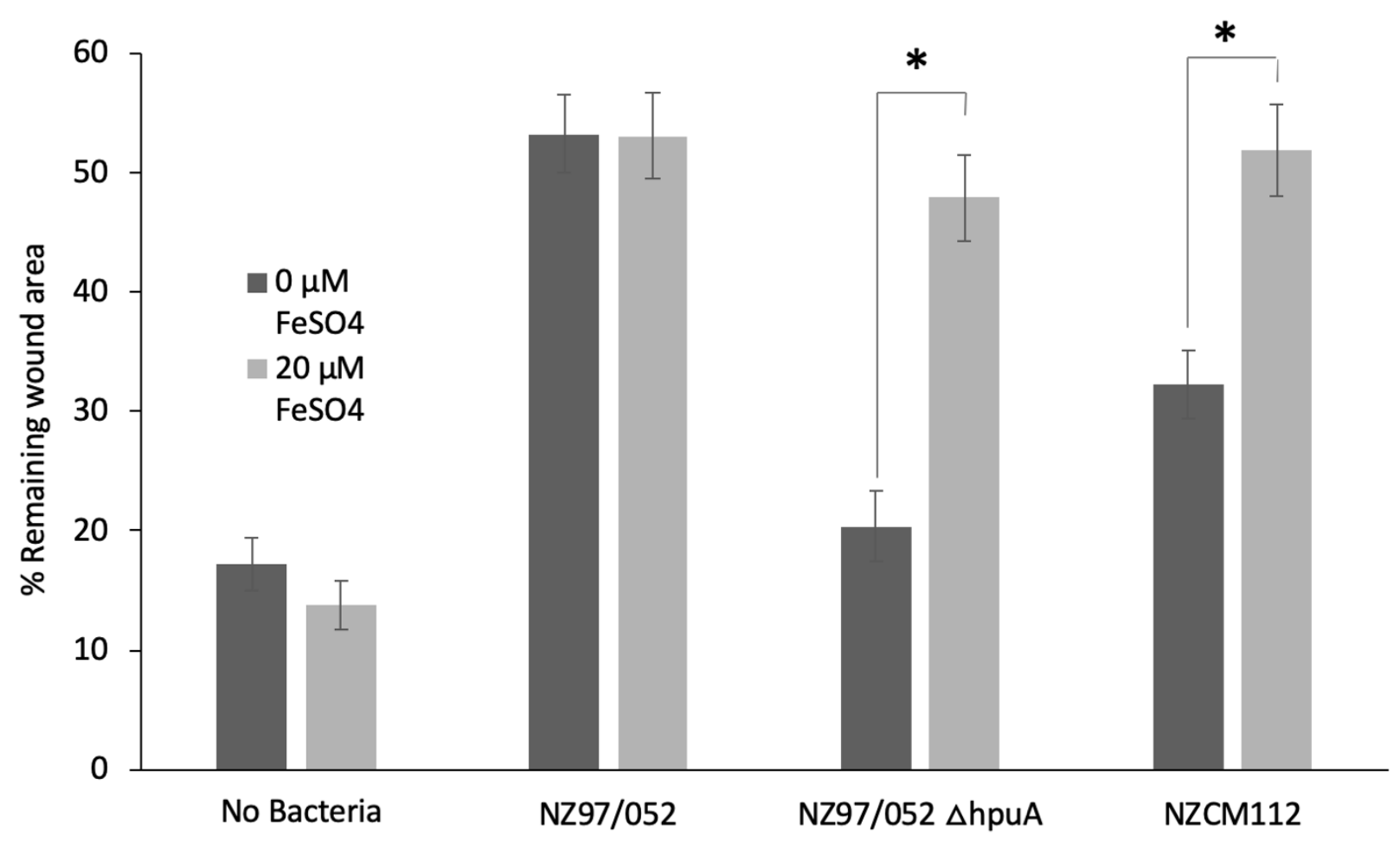

Figure 5.14. Addition of $20 \mu \mathrm{M} \mathrm{FeSO}$ to the cell culture medium resulted in cell migration inhibition by NZCM112 and NZ97/052 $\triangle$ hpuA. Iron supplementation significantly increased wound repair inhibition NZCM112 and NZ97/052 $\triangle h p u A$, without altering cell migration in uninfected cells or those infected with NZ97/052. Error bars show standard deviation. Data points are determined from means of eight replicates. Significant difference was determined with t-test. P-value $<0.05=*$.

\subsection{Discussion}

In Chapter Three, the NZ97/052 N. meningitidis household isolates were characterised. One of the isolates in this household, NZCM112, differed in the phenotype of cell migration inhibition. While NZ97/052 and NZCM111 were effective at inhibiting cell migration of 16HBE cells, NZCM112 was not, even though all three isolates were indistinguishable from one another by conventional laboratory typing. The aim of this chapter was to carry out genome and transcriptome analysis of the NZ97/052 household isolates in order to identify the meningococcal factor responsible for inhibition of host cell migration. Many genes in $N$. meningitidis have previously been tested in their link to inhibit cell migration (Appendix, Table 
9.2). These have included multiple surface factors known to aid in virulence and interact with host cells, i.e. type IV pili, opacity adhesions, bioactive components of the lipopolysaccharide, and other outer membrane components, but none had any effect on the inhibition of wound repair. With so many genes having been previously tested, a new approach to identify additional candidates was needed.

Genomic and transcriptomic profiles of isolates from the NZ97/052 household were analysed to identify transcripts with nucleotide variants, or which were differentially expressed between NZCM112 and NZ97/052. Whole-genome sequencing was completed for all three isolates of the NZ97/052 household. There were 49 total variants between these closely related isolates, and this number falls within the range previously reported for other related household isolates (Ren et al., 2017). Genome sequence comparisons revealed a deletion and base substitution mutation in hpuA, which resulted in a frameshift and a premature stop codon in NZCM112 only. The hpuA gene encodes one protein of a two component receptor, $\mathrm{HpuAB}$, which is involved in the acquisition of iron from haptoglobin complexed haemoglobin and allows $N$. meningitidis to thrive in the low-iron environment (Lewis \& Dyer, 1995; Rohde \& Dyer, 2004).

Transcriptome sequencing was completed for the household isolates which differ in the ability to inhibit cell migration, NZCM112 and NZ97/052. Gene expression data confirmed loss of expression of both hpuA and its co-transcribed partner, hpuB, in NZCM112. Conversely, in NZ97/052, higher expression of transcripts encoding $h p u A$ and $h p u B$ was observed. This result was expected, based on the genome sequence data. Genome analysis results also revealed a striking upregulation of multiple transcripts encoding proteins involved in iron acquisition, in NZCM112. The upregulated proteins included those involved in acquisition of iron from transferrin, lactoferrin, siderophores from other bacteria, and ferrous iron. This result was initially unexpected, as iron is well established to be important for bacterial virulence (Cornelissen \& Sparling, 1994; Ratledge \& Dover, 2000; Wooldridge \& Williams, 1993), and is known to be a critical factor in the pathogenesis of $N$. meningitidis (Perkins-Balding et al., 2004). Therefore, it was expected that disease-associated isolate NZ97/052 would have increased 
expression of genes involved in the acquisition of iron, considering its importance in the development of invasive disease. However, many of these proteins are upregulated in low-iron environments (Perkins-Balding et al., 2004). Presumably the loss of HpuAB in NZCM112 leads to the bacteria entering a low-iron state, and other mechanisms are upregulated to compensate for the loss and fulfil the iron requirements of $N$. meningitidis. Transferrin and lactoferrin are reported to be the main sources of iron from the serum and mucosa, respectively (Trivedi et al., 2011). It is intriguing to see these upregulated in the absence of HpuA, which is normally considered to be important for acquiring haem from haemoglobin in the bloodstream. This result suggests the HpuAB uptake system is acquiring iron in the in vitro cell culture model.

It is possible the different bacterial growth rates measured for NZ97/052 and NZCM112 (Figure 3.4, Figure 4.4) can be explained due to limitations of some key nutrients, such as iron, in NZCM112. However, it is clear that even upregulating many other iron acquisition proteins does not allow NZCM112 to grow as well as NZ97/052. HpuA expression is clearly important for bacterial growth and iron acquisition, but there may be a cost to expressing this protein; for instance this surface lipoprotein may have a high level of immunogenicity.

NZ97/052 was found to upregulate proteins involved in sulfate and cysteine transport and metabolism, relative to NZCM112. It appears higher iron levels require higher sulfur levels, although this phenomenon cannot currently be explained. Sulfur metabolism is known to be important in the nasopharyngeal colonisation for meningococci (Schoen et al., 2014), and it has previously been suggested that the sulfur metabolism pathway is important for diseaseassociated meningococcal isolates (Ren et al., 2017). However, to our knowledge, this is the first indication that importing and metabolising sulfur is a response to higher iron availability. One possibility is the bacteria are making more enzymes containing iron-sulfur (Fe-S) clusters, which are key in respiration and metabolism (Brzoska et al., 2006; Johnson et al., 2005). Another possible explanation is that NZ97/052 may have increased levels of intracellular iron, which could result in increased free radicals, via the Fenton reaction (Schafer et al., 2000). The cysteine biosynthesis pathway is involved in sulfur acquisition and leads to the production of 
glutathione (Schoen et al., 2014). Glutathione is a stress response protein which can reduce oxidative stress and prevent cellular damage (Rahman et al., 2005). The NZ97/052 isolate may increase the import of sulfur in order to synthesise glutathione, which in turn could be providing antioxidant protection. Previous experiments completed in our laboratory have linked levels of intracellular glutathione with expression of genes involved in sulfur acquisition in isolates belonging to the NZ97/192 household (Appendix, Section 9.4). A glutathione assay could be carried out to compare levels of intracellular glutathione between NZCM112 and NZ97/052, to further investigate this hypothesis.

The first step to determine the meningococcal factor responsible for wound repair was to identify a panel of candidate genes which could have an impact on cell migration of respiratory epithelial cells. The genome and transcriptome profiles were used to identify a panel of candidate genes which could have an impact on cell migration of respiratory epithelial cells. Comparison of the genome and transcriptome sequence profiles of NZCM112 and NZ97/052, which differ in their ability to inhibit wound repair, identified four candidate genes $h p u A, h p u B$, $f e t A$, and fur. The variant in $h p u A$, identified from genomic analysis, is the only variant that resulted in the loss of expression of a protein. Transcriptome comparisons confirmed the two genes, hpuA and $h p u B$, were expressed in NZ97/052, but not NZCM112. Therefore, these genes were identified as highly interesting candidates. The gene fur encodes an iron regulator, therefore, this was selected as a candidate due to the high number of iron-regulated genes differing in expression between the isolates. Evidence described in Chapter Four suggested that iron or iron-regulated proteins may play a role in wound repair inhibition by $N$. meningitidis (Figure 4.2). A final candidate, fetA, was downregulated in NZ97/052. This gene was selected as it was the most differentially expressed gene and is known to be expressed on the surface of $N$. meningitidis. Outer membrane proteins present in NZ97/052 were of particular interest, as these are most likely to be interacting with host cells. Some other outer membrane proteins of interest, such as opa, could not be selected as a candidate for genome deletion, as there are multiple copies present in the genome. Deletion of more than one unlinked gene was not feasible, as multiple antibiotic resistance cassettes were not available for this project. 
The candidate genes were deleted by allelic replacement to determine if they were required for cell migration inhibition. N. meningitidis is naturally competent, therefore mutants were made by introducing a PCR fusion product containing an antibiotic resistance cassette flanked by sequences corresponding to the chromosomal regions on either side of the targeted gene. Transformation of this product resulted in homologous recombination, with the gene of interest replaced by an antibiotic resistant cassette. Allelic replacements of fet $A, f u r, h p u A, h p u B$, and hpuAB were created in the NZ97/052 isolate, with the goal of generating a more carriage-like isolate by revoking the ability to inhibit wound repair. Mutants were selected by their ability to grow in the presence of kanamycin, and gene deletions were validated by PCR, to confirm the correct gene region size, and Sanger sequencing of the antibiotic resistance cassette junctions was also undertaken. Gene deletion mutants were tested for their ability to inhibit wound repair by using cell migration assays and comparing them to wild-type parent isolates. There was no correlation between wound repair inhibition and deletion of fetA, fur, or hpuB; removal of these genes resulted in mutants that inhibited migrating $16 \mathrm{HBE}$ cells at a similar level as the wild-type NZ97/052 parental isolate. However, deletion of the hpuA gene or hpuAB together both similarly completely nullified the ability of NZ97/052 to inhibit host cell wound repair. This exciting result identifies $h p u A$ as the meningococcal factor responsible for cell migration inhibition in NZ97/052.

Validation of gene deletion involves complementation with a wild-type copy of the parent gene, to see if the wild-type phenotype is restored. Complementation can demonstrate that a change in phenotype is due to the deletion of the specific gene and is not the result of disruption of a downstream gene or insertion of the antibiotic resistance cassette in more than one locus. Unfortunately there were no available plasmids that replicate in N. meningitidis which could be used for this purpose. To dissect the relative impact of $h p u A$ and $h p u B$, two genes that are situated in an operon and which are both expressed only in NZ97/052, deletions of both genes were made individually, along with a double mutant, $h p u A B$. Deletion of $h p u A$ has been reported to result in a lack of expression of downstream gene $h p u B$ (Chen et al., 1998); this effect was also seen in the genome and transcriptome data of NZ97/052 and NZCM112 (Table 
4.2 and Figure 4.4). These results confirm that deletion of only $h p u B$ does not affect expression of $h p u A$, as the $\triangle h p u B$ mutant had a different phenotype from the $\triangle h p u A B$ double mutant. Results from deleting $h p u A, h p u B$, and $h p u A B$ reveal the change in wound repair inhibition phenotype is due to the loss of HpuA, not HpuB.

Strikingly, when migrating $16 \mathrm{HBE}$ cells infected with NZ97/052 $\Delta h p u A$ were supplemented with iron sulfate, the phenotype of cell migration inhibition could be almost completely rescued (Figure 5.14). In the presence of iron, the hpuA deletion mutant regained the ability to inhibit wound repair. One explanation for this unexpected result is that there is a second meningococcal factor, induced by the presence of iron, which mediates inhibition of cell migration. The impact of iron supplementation on NZ97/052 $\triangle h p u A$ is the same as was seen for other carriage associated isolates; in all cases there was an increase in inhibition of host cell migration (Figure 4.3). Further experiments would need to be completed to investigate the prevalence of this second factor which inhibits cell migration in the presence of iron. This secondary factor clearly exists in NZ97/052, but it appears to be masked when HpuA is present. Identification of this second meningococcal factor will be a focus of future studies. In order to detect candidate genes that are upregulated in the presence of iron and which may affect wound repair inhibition, expression profiles of the NZ97/052 $\triangle h p u A$ mutant with and without the addition of iron could be analysed. Another future experiment could generate a fur deletion mutant in the NZCM112 isolate background, and determine if iron supplementation still results in an increase in cell migration inhibition. This experiment would demonstrate whether this secondary factor is regulated by Fur. Fur has been identified to upregulate multiple $N$. meningitidis genes with the addition of iron (Grifantini et al., 2003). Some of these upregulated genes include outer membrane proteins, such as NspA and AniA, which could be candidates for this secondary meningococcal factor. Other candidate genes include ferrous iron transporter proteins, located in the outer membrane and potentially interacting with $16 \mathrm{HBE}$ cells. Results presented in this chapter demonstrate the utility of examining closely related meningococcal isolates that differ phenotypically. Further examination of the role of $\mathrm{HpuA}$ is required to 
determine the mechanism of the effect on wound repair inhibition, and is addressed in the next chapter.

\subsection{Summary}

In summary, genome and transcriptome sequence data, for related household isolates that differed in their ability to inhibit wound repair, were mined to identify a handful of candidate genes potentially responsible for that phenotypic difference. Candidate genes were deleted from NZ97/052 and the mutants were compared to wild-type parent strains in cell migration assays. Deletion of $h p u A$, a gene that encodes a haptoglobin-haemoglobin binding protein, from the disease-associated isolate, NZ97/052, nullified the ability to inhibit epithelial cell migration. These exciting results suggests a novel function for HpuA. In addition to its role in iron acquisition, it is also the meningococcal factor that is responsible for cell migration inhibition. It clearly plays a key role in the interaction between the host and pathogen that may lead to invasive disease. Furthermore, supplementation with iron sulfate of migrating $16 \mathrm{HBE}$ cells infected with the NZ97/052 $\triangle h p u A$ deletion mutant rescued the phenotype, and significantly

increased the degree of cell migration inhibition. These results support findings in Chapter Four that implicate iron in the inhibition of cell migration by $N$. meningitidis. These results indicate there is likely a second gene which can affect wound repair inhibition; this second gene will be the focus of future studies. The next goal for this project, however, was to further investigate how expression of HpuA impacts wound repair inhibition by N. meningitidis. 


\section{The role of HpuA on the inhibition of respiratory epithelial cell wound repair}

\subsection{Introduction}

Iron is an essential element for nearly all living organisms and homeostasis to maintain iron levels is critical (Anderson \& Frazer, 2017; Wallace, 2016). Humans and other mammals utilise iron-binding proteins and iron-sequestering compounds to regulate free iron and keep levels low to prevent the growth of invading pathogens, a strategy termed "nutritional immunity" (Wallace, 2016).

Approximately $65 \%$ of iron in the human body is stored within haemoglobin (Andrews, 1999). Haemoglobin is normally found inside red blood cells, but lysis of these cells can occur during acute inflammation or due to the action of haemolytic toxins (Jeney et al., 2014; Quaye, 2015). Free haemoglobin is bound by haptoglobin to form haptoglobin-haemoglobin complexes (Wassell, 2000). Formation of this complex prevents scavenging microbes from utilising haemoglobin as an iron source and limits oxidative damage by haemoglobin to specific organs, such as the kidneys (Lim et al., 1998; Tseng et al., 2004). Haptoglobin can also protect against the toxicity of haemoglobin. It does this by facilitating haemoglobin removal through endocytosis by the macrophage scavenger receptor CD163 (Kristiansen et al., 2001; Nielsen \& Moestrup, 2009; Schaer et al., 2014). Hepatic cells in the liver are mainly responsible for the production of haptoglobin, however non-hepatic cells have been stimulated to produce haptoglobin in vitro, including kidney, lung, and skin cells (D'Armiento et al., 1997; de Kleijn et al., 2002; Piva et al., 2001; Yang et al., 1995). Haptoglobin is considered an acute phase protein and is synthesised in response to inflammation (de Kleijn et al., 2002; Shih et al., 2014; Wang et al., 2001). It has long been known that haptoglobin has some antimicrobial activity (Dobryszycka, 1997; Eaton et al., 1982), and recent studies have demonstrated that haptoglobin is bacteriostatic against some Gram-negative bacteria (Board-Davies et al., 2015). 
Cell migration is an extremely important early step in wound repair. A previous study, investigating arterial tissue wound repair, linked expression of haptoglobin to increased cell migration (de Kleijn et al., 2002). Garibay-Cerdenares et al also found that adding purified haptoglobin to migrating cells significantly accelerated wound healing of epithelial cells (2015). Other research has characterised the interaction of haptoglobin with the CCR2 chemokine receptor (Garibay-Cerdenares et al., 2015; Maffei et al., 2009). CCR2 chemokine receptors recognise CCL2 chemokines (Fang et al., 2012), and expression of this receptor has been demonstrated in epithelial cells (Christensen et al., 2004; Elner et al., 1991). CCL2 chemokines contribute to cell migration by interacting with CCR2 receptors on tumour cells (Chiu et al., 2012). Increased expression of CCR2 has been linked to the progression of cancer metastasis in breast cancer cells, and is consequently thought to be important in cell migration (Lim et al., 2016; Yao et al., 2018). Haptoglobin has been shown to induce migration of ovarian cancer cells, but when CCR2 receptors were blocked with a synthetic antagonist, the effect of haptoglobin on cell migration disappeared (Garibay-Cerdenares et al., 2015). Results from these studies suggest that haptoglobin may induce changes in the actin cytoskeleton, cell morphology, and the migration of cells. There is currently a lack of research investigating the role of meningococcal binding of haptoglobin at the mucosal surface. In the context of wound repair inhibition by $N$. meningitidis, disease-associated bacterial isolates may bind haptoglobin-haemoglobin complexes with the meningococcal HpuAB receptor, sequestering haptoglobin from host cells and inhibiting cell migration.

\subsubsection{HpuAB - A Neisseria haptoglobin-haemoglobin receptor}

Bacteria that are adapted to human colonisation, including pathogenic Neisseria, produce numerous transporters for host iron-containing proteins, including transferrin, lactoferrin and haem (Perkins-Balding et al., 2004; Weinberg, 2009). Transferrin and lactoferrin are both important sources of iron for N. meningitidis, and all clinical isolates have lactoferrin-binding proteins, which are not phase variable (Archibald \& DeVoe, 1980; Biville et al., 2014). Pathogenic Neisseria are able to use haemoglobin, haptoglobin-haemoglobin, and haem as iron sources for growth (Dyer et al., 1987). N. meningitidis utilises haemoglobin as an iron source via 
HpuAB or HmbR (Stojiljkovic et al., 1996), which are both TonB-dependent, phase variable haemoglobin surface receptors (Chen et al., 1998; Lewis et al., 1999; Tauseef et al., 2011). HpuAB is a bipartite outer membrane protein which acquires iron from haemoglobin and haptoglobin-haemoglobin, whereas HmbR lacks an accessory lipoprotein and is able to bind haemoglobin, but not haptoglobin or haptoglobin-haemoglobin complexes (Rohde \& Dyer, 2004; Stojiljkovic et al., 1995; Stojiljkovic et al., 1996).

Some meningococcal isolates encode both transporters, while others encode only one of either $\mathrm{HpuAB}$ or $\mathrm{HmbR}$. These genes can be switched on or off by phase variation, but there are also many strains of N. meningitidis that completely lack both genes (Chen et al., 1998; Chen et al., 1996; Lewis \& Dyer, 1995; Lewis et al., 1997; Stojiljkovic et al., 1996). Expression of HmbR, or both $\mathrm{HmbR}$ and $\mathrm{HpuAB}$, is overrepresented in disease isolates; $99 \%$ of disease-associated meningococcal strains encode at least one haemoglobin transporter. In an examination of meningococcal strains belonging to clonal complexes which cause the highest rates of disease, 90\% contain both $\mathrm{HpuAB}$ and $\mathrm{HmbR}$, however, expression of only $\mathrm{HpuAB}$ is underrepresented in disease isolates (Tauseef et al., 2011). This may suggest that HmbR is more efficient at acquisition of iron during systemic spread, or perhaps the HpuA surface lipoprotein has a high level of immunogenicity. HpuAB is a two component receptor, which is composed of the 42-kDa HpuA lipoprotein and the 89-kDa outer membrane protein HpuB (Chen et al., 1998). In N. meningitidis, expression of $\mathrm{HpuAB}$ allows the bacteria to thrive in a low-iron environment (Lewis \& Dyer, 1995; Rohde \& Dyer, 2004). HpuA and HpuB are co-regulated and encoded by a twogene operon; deletion of $h p u A$ has been shown to abrogate the expression of $h p u B$, suggesting these genes are co-transcribed (Chen et al., 1998).

A serogroup A N. meningitidis isolate, which caused an accidental laboratory-acquired infection, was analysed for genetic and phenotypic modifications before and after human passage (Omer et al., 2011). Analysis showed the isolate, collected from a blood sample of the infected laboratory worker, expressed a different haemoglobin iron receptor (HpuAB) than the parental isolate ( $\mathrm{HmbR})$. The isolate from the blood had HmbR in the phase variable "off" state, while 
HpuAB had switched to the phase variable "on" state, which was opposite from the original cultured isolate (Omer et al., 2011). This case of accidental human passage may implicate $\mathrm{HpuAB}$, over $\mathrm{HmbR}$, in invasive disease or bacterial survival within the bloodstream. The NZ97/052 isolate, investigated in this thesis, expresses HpuAB and was also cultured from a blood sample of a patient with meningococcal disease. The closely related NZCM112 isolate, collected the nasal swab of an asymptomatic household contact, has a mutation in HpuA, resulting in a premature stop codon. It is possible HpuAB may play a role in bacterial virulence.

HpuAB is expressed as an iron acquisition system, not only in $N$. meningitidis but also in Neisseria gonorrhoeae (Chen et al., 1998; Chen et al., 1996; Cornelissen \& Hollander, 2011). $\mathrm{HpuAB}$ is likely the main haemoglobin receptor of gonococci, as the $h m b R$ gene contains a premature stop codon in all N. gonorrhoeae isolates tested (Chen et al., 1996; Stojiljkovic et al., 1996). These findings could indicate that HpuA plays an important role in the mucosal epithelium, and it is clear this transporter must be further investigated. Understanding the full role of $N$. meningitidis HpuA in the mucosal epithelial surface could have a number of useful implications for other bacteria with haptoglobin-haemoglobin transporters, for instance; $N$. gonorrhoeae or non-typeable $H$. influenzae, which causes ear infections.

\subsubsection{Aims of the chapter}

The aims of this chapter were to further characterise the hpuA deletion mutant, investigate the possible effect of haptoglobin on cell migration, and to shed light on the mechanism by which hpuA affects wound repair inhibition. There were multiple leading hypotheses about the role of haptoglobin in wound repair. Adding haptoglobin directly to an ovarian cancer cell line has been shown to increase the rate of cell migration (Garibay-Cerdenares et al., 2015). To investigate whether this phenomenon occurred in 16HBE airway epithelial cells, the impact of added haptoglobin on migrating host cells was assessed. Enzyme linked immunosorbent assays (ELISAs) were used to determine whether 16HBE cells secreted haptoglobin in response to meningococcal isolates, or if the bacteria directly or indirectly reduced haptoglobin levels. 
Fluorescent microscopy was used to investigate if disease-associated isolates were co-localised with intracellular haptoglobin, or if the presence of HpuA altered host cell morphology. Haptoglobin sensitivity assays were carried out to detect whether haptoglobin had any detectable antimicrobial activity against $N$. meningitidis. Growth assays were also conducted to measure any significant differences in haptoglobin sensitivity or bacterial growth between NZ97/052 and the NZ97/052 $\Delta$ hpuA mutant.

Association and invasion assays were completed to detect differences between $N$. meningitidis isolates, and the differences in cell association by the wild-type and hpuA mutant were further investigated using fluorescence microscopy. Finally, HpuA was heterologously expressed in $E$. coli to determine whether HpuA alone is sufficient to mediate bacterial attachment to host cells. These experiments were designed in order to determine the mechanism of HpuA on wound repair inhibition by $N$. meningitidis.

\subsection{Results}

\subsubsection{Human haptoglobin supplementation has a slight impact on wound repair inhibition by NZ97/052 and NZCM112.}

In experiments described in Chapter Five, supplementation of cell migration assays with iron increased the ability of NZCM112 and NZ97/052 $\triangle h p u A$ to inhibit cell migration (Figure 5.14). It was investigated if purified human haptoglobin, as a potential source of iron for both bacteria and cells, could alter the ability of the meningococcal isolates to inhibit cell migration. In the absence of $N$. meningitidis, ovarian cancer cells have been reported to migrate more rapidly when supplemented with purified haptoglobin (Garibay-Cerdenares et al., 2015). Oris cell migration assays were carried out with human haptoglobin supplementation to determine whether haptoglobin might overcome the inhibition of cell migration by meningococcal isolates and cause $16 \mathrm{HBE}$ cells to migrate more rapidly, or whether haptoglobin may increase cell migration inhibition by NZCM112 and NZ97/052 $\triangle h p u A$ in a similar manner to iron sulfate and iron chloride (Figure 4.2). 
Increasing concentrations of haptoglobin $(0 \mathrm{ng} / \mathrm{mL}, 10 \mathrm{ng} / \mathrm{mL}$, and $1000 \mathrm{ng} / \mathrm{mL})$ were added to uninfected or infected migrating respiratory epithelial cells (Figure 6.1). The 16HBE cells were infected with NZ97/052, an HpuA-expressing isolate, NZCM112 and the NZ97/052 $\Delta h p u A$ mutant isolate, which lacks HpuA. Addition of $10 \mathrm{ng} / \mathrm{mL}$ or $1000 \mathrm{ng} / \mathrm{mL}$ haptoglobin had no significant effect on uninfected $16 \mathrm{HBE}$ cells compared to cells with no added haptoglobin ( $p=$ 0.282 and $p=0.334$, respectively). Supplementation with haptoglobin caused a slight increase in cell migration inhibition by NZ97/052 $\triangle$ hpuA isolate, which appeared to be dose-dependent. Over 16 hours, 16HBE cells infected with NZ97/052 $\triangle$ hpuA migrated to leave $16.88 \%$ of the initial wound area. The addition of $10 \mathrm{ng} / \mathrm{mL}$ haptoglobin caused cell migration inhibition to increase slightly to $20.01 \%$ initial wound area $(p=0.023$ ). Addition of $1000 \mathrm{ng} / \mathrm{mL}$ haptoglobin caused slightly higher levels of cell migration inhibition, leaving $22.44 \%$ initial wound area, which was significantly higher than $0 \mathrm{ng} / \mathrm{mL}$ haptoglobin supplementation $(\mathrm{p}<0.01)$. 16HBE cells infected with the NZCM112 isolate showed a similar trend with haptoglobin supplementation, however this did not reach statistical significance $(p=0.132)$.

The HpuA-expressing NZ97/052 isolate appeared to have decreased ability to inhibit wound repair with increasing concentrations of haptoglobin. Without haptoglobin supplementation, the NZ97/052 isolate inhibited cell migration to leave $42.45 \%$ of the initial wound area. This cell migration inhibition was slightly lower when $1000 \mathrm{ng} / \mathrm{mL}$ haptoglobin was added, which resulted in a wound area which was $39.42 \%$ of the initial size $(p<0.001)$.

These results suggest haptoglobin supplementation causes a slight increase in cell migration inhibition by the NZ97/052 $\triangle h p u A$ isolate. However, this effect is not as striking as the increase in wound repair inhibition seen with the addition of iron sulfate, which caused a 2.3 fold increase in the percent of remaining wound area (Figure 5.14). In addition, purified haptoglobin did not significantly alter the migration of uninfected $16 \mathrm{HBE}$ cells, in contrast with the reported effect on ovarian cells (Garibay-Cerdenares et al., 2015). 


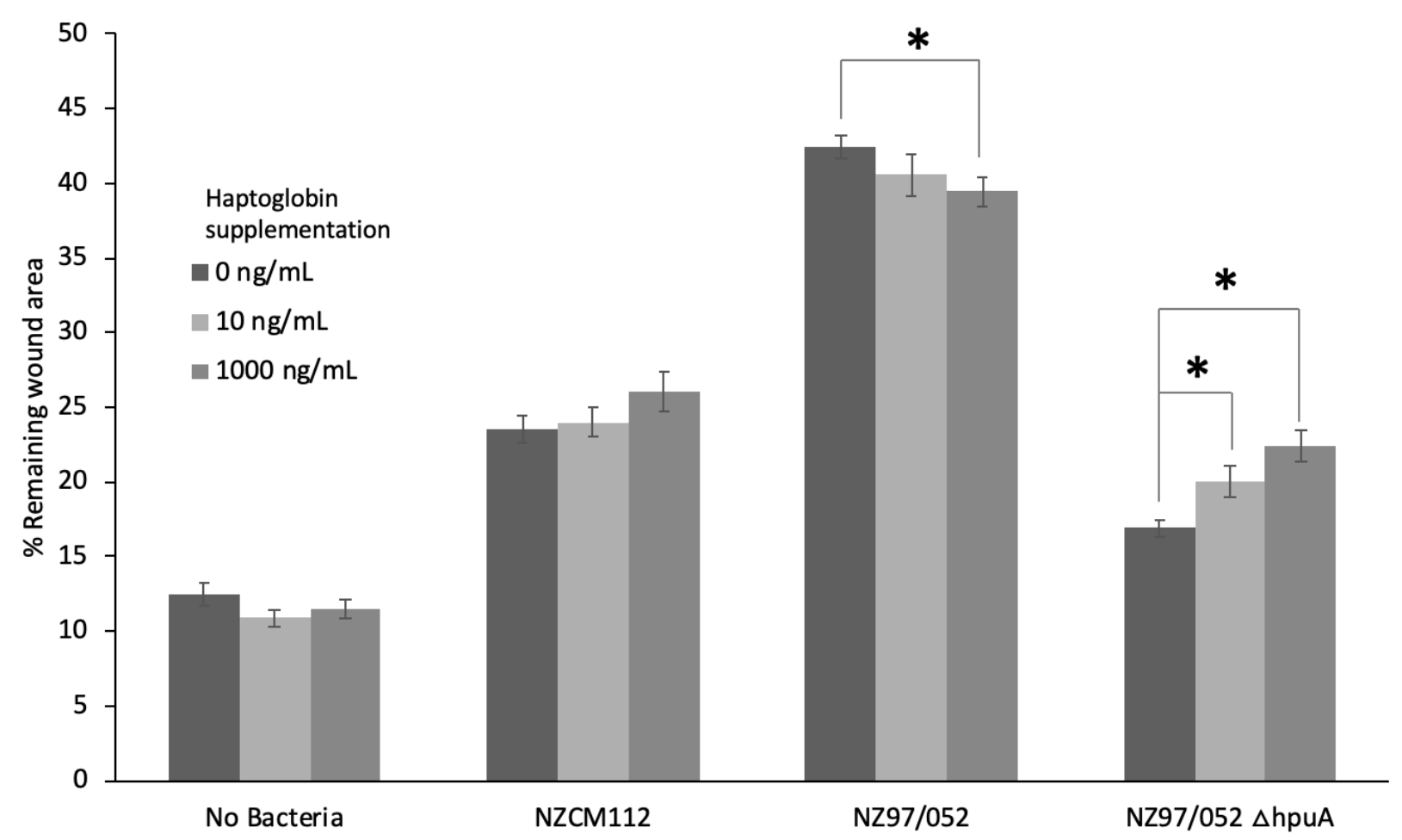

Figure 6.1. Haptoglobin supplementation increases the ability of NZ97/052 $\triangle h p u A$ to inhibit wound repair. Oris cell migration assays of $16 \mathrm{HBE}$ cells co-cultured with meningococcal isolates were supplemented with increasing concentrations of human haptoglobin. After 16 hours, the remaining wound area was compared to the initial wound area. Error bars show standard deviation. Data points are determined from means of eight replicates. Significant difference was determined using a t-test. P-value $<0.05=*$.

\subsubsection{Haptoglobin levels remain constant in bacteria-host cell co-culture}

HpuA is a haptoglobin-haemoglobin binding protein, therefore it was hypothesised that haptoglobin may be playing an important role in wound repair. To determine whether secreted haptoglobin was induced in host cells or consumed by the bacterial isolates at different rates, a human-specific haptoglobin enzyme linked immunosorbent assay (ELISA) was used. $N$. meningitidis is also able to use bovine haemoglobin as an iron source (Lee \& Hill, 1993). Bovine haptoglobin-haemoglobin is abundant in the FBS, which was added to the M199 medium, therefore some of the haptoglobin in the system may be of bovine origin. To control for this, a bovine-specific haptoglobin ELISA was also carried out. 
Respiratory epithelial cells were grown to confluence in 24 well plates, and overnight cultures of NZCM112, NZ97/052, and NZ97/052 $\triangle h p u A$ were used to infect the $16 \mathrm{HBE}$ cells at an MOI of approximately 10. An uninfected control was also used. Host cell and meningococcal co-cultures were incubated at $37^{\circ} \mathrm{C}$ and supernatant was collected to measure haptoglobin concentrations at zero, two, four, eight, 12 and 24 hours post-infection. Samples were centrifuged to remove bacteria and haptoglobin bound to the bacteria.

There was no apparent difference in human haptoglobin concentrations between uninfected and infected $16 \mathrm{HBE}$ cells, or between cells infected with various meningococcal isolates (Figure 6.2A). Bovine haptoglobin levels also remained steady for uninfected and infected $16 \mathrm{HBE}$ cell systems throughout the course of the experiment (Figure 6.2B). Free haptoglobin levels in the system did not appear to change over time; not enough haptoglobin bound to $N$. meningitidis over 24 hours to noticeably reduce the level of free haptoglobin in the supernatant. Diseaseassociated and carriage-associated isolates did not significantly differ in their effects on the levels of secreted haptoglobin.

There was no increase in levels of human haptoglobin detected upon addition of any of the bacterial isolates, suggesting that secretion of haptoglobin by $16 \mathrm{HBE}$ cells is not induced by $N$. meningitidis. This experiment did not enable us to discern whether $N$. meningitidis is able to utilise haem from haptoglobin-haemoglobin complexes, however, there was no evidence that loss or consumption of haptoglobin is occurring or leading to reduced cell migration. Secreted haptoglobin levels, as measured by ELISA, did not increase with the addition of $N$. meningitidis, and haptoglobin did not appear to be directly or indirectly destroyed by the bacteria. 

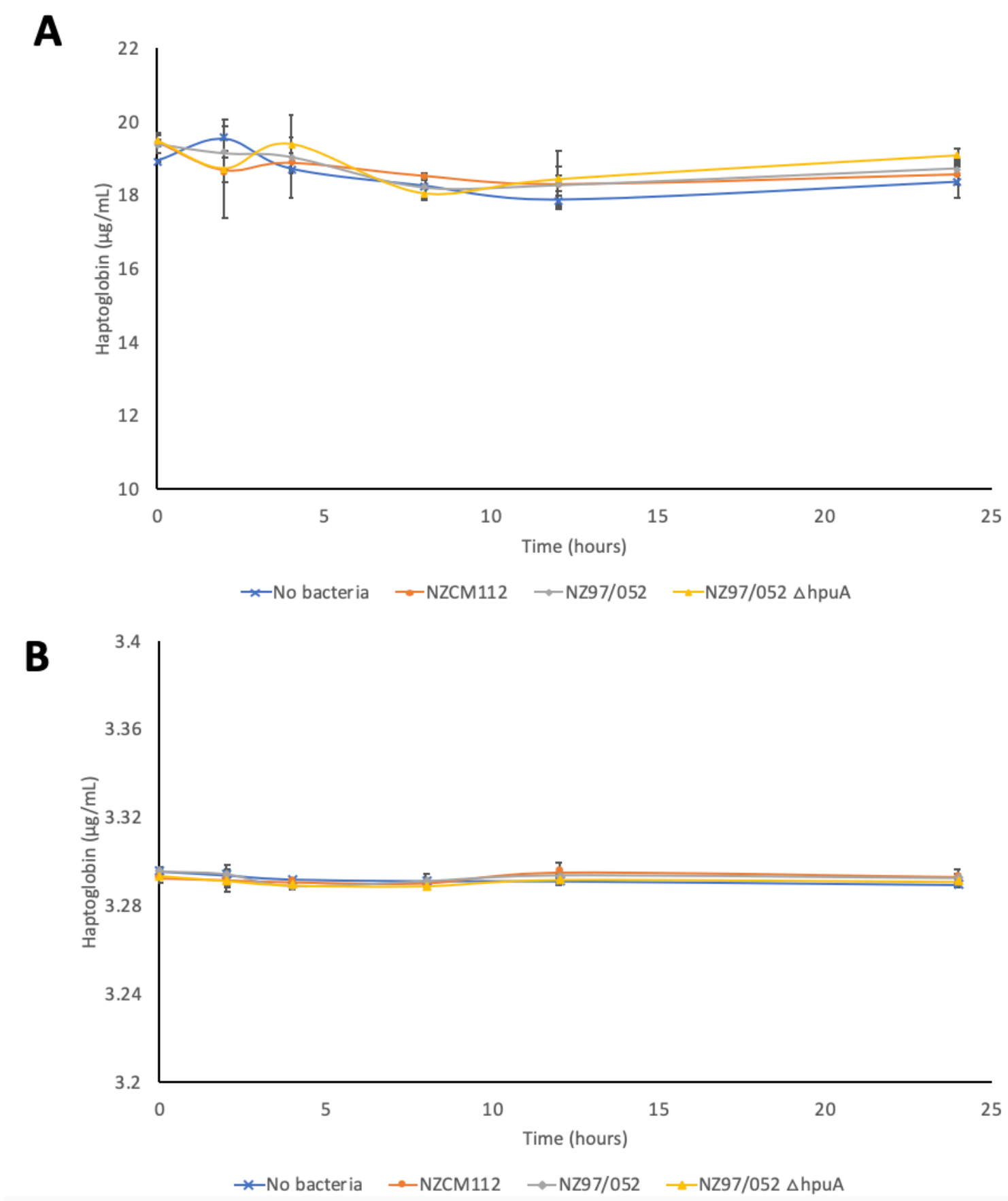

Figure 6.2. Human and bovine haptoglobin levels remain constant in bacteria and host cell co-culture. (A) Human haptoglobin levels remained constant in the co-culture supernatant over time. No significant differences were seen between uninfected or infected $16 \mathrm{HBE}$ cells, or between $16 \mathrm{HBE}$ cells infected with different isolates. (B) Bovine haptoglobin levels remained stable over 24 hours, and did not differ between co-culture samples with different meningococcal isolates. Each meningococcal isolate was assayed in parallel with at least three replicates for each time point. Error bars represent standard deviation. 16HBE cells were uninfected (blue) or infected with NZCM112 (red), NZ97/052 (grey) or NZ97/052 $\triangle h p u A$ (yellow). 


\subsubsection{Fluorescence microscopy identified higher numbers of cell association of NZ97/052 than NZCM112 and NZ97/052 $\triangle$ hpuA}

HpuA binds haptoglobin-haemoglobin, therefore, it was theorised that $N$. meningitidis isolates, which express HpuA, could be co-localised with intracellular haptoglobin when bound to host cells. Fluorescent staining of cell nuclei and human haptoglobin was paired with microscopy to investigate any co-localisation. Confluent 16HBE cells were infected with NZCM112, NZ97/052, or NZ97/052 $\triangle h p u A$ isolates at an MOI of approximately 10 . Immediately following infection, 10 $\mu \mathrm{L}$ was removed from each well and plated on CBA plates to ensure comparable numbers of bacteria were added to each well. Bacteria and host cell co-cultures were incubated for four hours to allow for attachment, before being washed with PBS and fixed with paraformaldehyde. DAPI was used to stain the nuclei of $16 \mathrm{HBE}$ cells and $N$. meningitidis. The fixed cells were then incubated with a primary human specific haptoglobin antibody. The next day, a secondary antibody, Alexa Fluor 488, was used to visualise haptoglobin location. Controls lacking primary or secondary antibodies were tested and no fluorescence was visualised for either control.

Representative images from fluorescence microscopy are shown in Figure 6.3. Examination of haptoglobin location suggests there may be a difference between $16 \mathrm{HBE}$ infected with NZ97/052 compared with host cells infected with NZCM112 and NZ97/052 $\Delta h p u A$. The images suggest there may be more cytoplasmic haptoglobin when NZ97/052 are present. Interestingly, the haptoglobin appeared to have a more nuclear location when cells were infected with NZ97/052 $\triangle h p u A$. Overlaying the DAPI and haptoglobin channels also gives the impression that there may be co-localisation between haptoglobin and NZ97/052 isolates. Examination of the DAPI staining revealed the most striking results from this assay. It was apparent that NZ97/052 had much higher numbers of bacteria adhering to host cells than NZCM112 or NZ97/052 $\Delta h p u A$. The differences in the numbers of adherent bacteria make it difficult to draw solid conclusions about any haptoglobin localisation.

There could be several explanations for the differing numbers of adherent bacteria. Haptoglobin may be having an antimicrobial effect and could be preventing growth of isolates without HpuA. 
It is also possible NZ97/052 is significantly outgrowing the other two isolates; results from Chapter Three (Figure 3.4) showed NZCM112 had significantly reduced bacterial growth, compared with NZ97/052. A final possible explanation is there may be differences in the adherence of the isolates to epithelial cells. Evidence in Chapter Three (Figure 3.6) indeed revealed NZCM112 adheres to epithelial cells at a reduced level compared to NZ97/052.

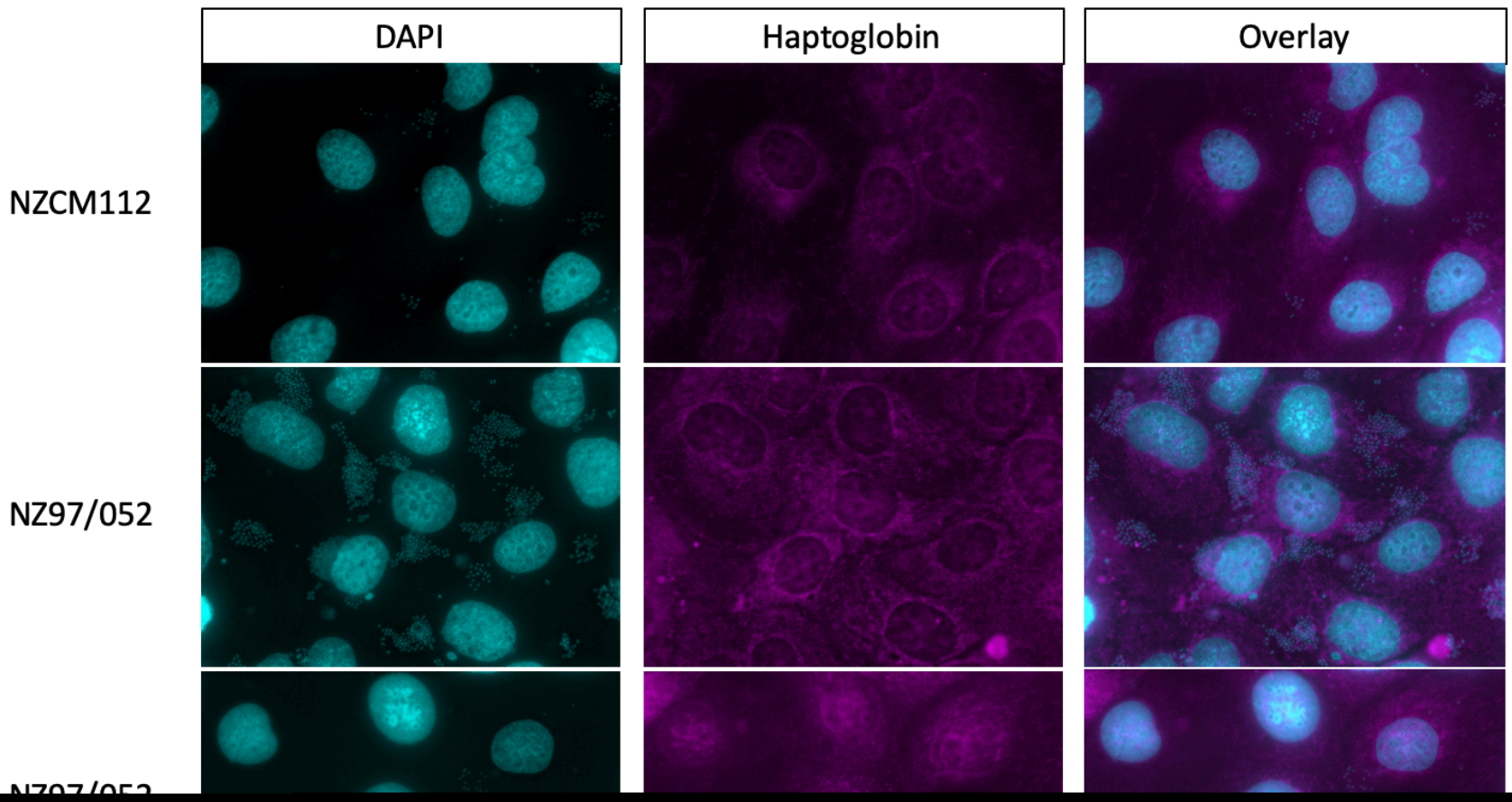

Figure 6.3. Fluorescence microscopy showing haptoglobin localisation in 16HBE and bacteria co-culture. DAPI stained nuclei and haptoglobin was visualised with primary haptoglobin antibody and secondary antibody Alexa Fluor 488. Images were merged using ImageJ. Scale bar, $20 \mu \mathrm{m}$. 


\subsubsection{Haptoglobin does not exhibit an antimicrobial effect on $\mathbf{N}$. meningitidis isolates}

Haptoglobin has previously been reported as exhibiting an antimicrobial effect on Gramnegative bacteria. Specifically, it has been shown to be bacteriostatic against Proteus mirabilis and Pseudomonas aeruginosa (Board-Davies et al., 2015). Fewer of the meningococcal isolates that lack HpuA were observed in contact with epithelial cells (Figure 6.3); a result that could be explained if haptoglobin is bacteriostatic against non-hpuA containing meningococci. $N$. meningitidis, along with many other bacterial pathogens, is able to use haptoglobinhaemoglobin complexes as an iron source. Antimicrobial activity of haptoglobin against these bacterial species has not, to our knowledge, been reported. To test whether haptoglobin has an antimicrobial effect on $N$. meningitidis isolates in the presence of host cells, a method similar to that previously described by Board-Davies et al was used (2015). Meningococci were incubated in the presence of confluent $16 \mathrm{HBE}$ cells in a 24-well plate. Prior to infection with $N$. meningitidis, respiratory epithelial cells were washed with PBS and incubated in serum-free M199 medium for 24 hours before fresh M199 medium with 10\% FBS was added back to the wells. Overnight CBA plate cultures of NZCM112, NZ97/052, and NZ97/052 $\triangle$ hpuA were used to infect $16 \mathrm{HBE}$ cells at an estimated $\mathrm{MOI}$ of 10 . At the same time, increasing concentrations of purified human haptoglobin, ranging from $0 \mathrm{ng} / \mathrm{mL}$ to $1000 \mathrm{ng} / \mathrm{mL}$, were added to the cocultures. After 24 hours of co-incubation, viable bacteria were determined by CFU counts. The initial inoculant was also measured by CFU counts. Growth of each strain in the presence of haptoglobin was normalised against growth in the absence of added haptoglobin $(0 \mathrm{ng} / \mathrm{ml}$ human haptoglobin). In some cases, added human haptoglobin increased meningococcal growth, but none of the differences in bacterial growth were significant at any concentration of haptoglobin (Figure 6.4). In contrast to the published results of Board-Davies et al (2015), where the highest haptoglobin concentration $(1000 \mathrm{ng} / \mathrm{ml})$ reduced bacterial growth for $P$. mirabilis and $P$. aeruginosa to $50 \%$ of what was seen without haptoglobin, inhibition of growth was not seen for any of the isolates, regardless of whether they expressed HpuA. Thus bacteriostatic 
actions of haptoglobin are unlikely to explain the reduced number of bacteria seen in the fluorescence microscopy images (Figure 6.3).

A

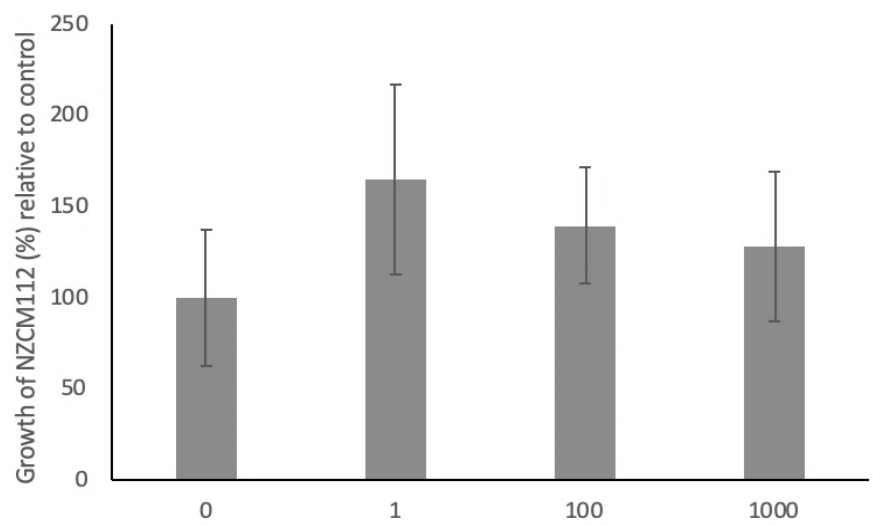

B

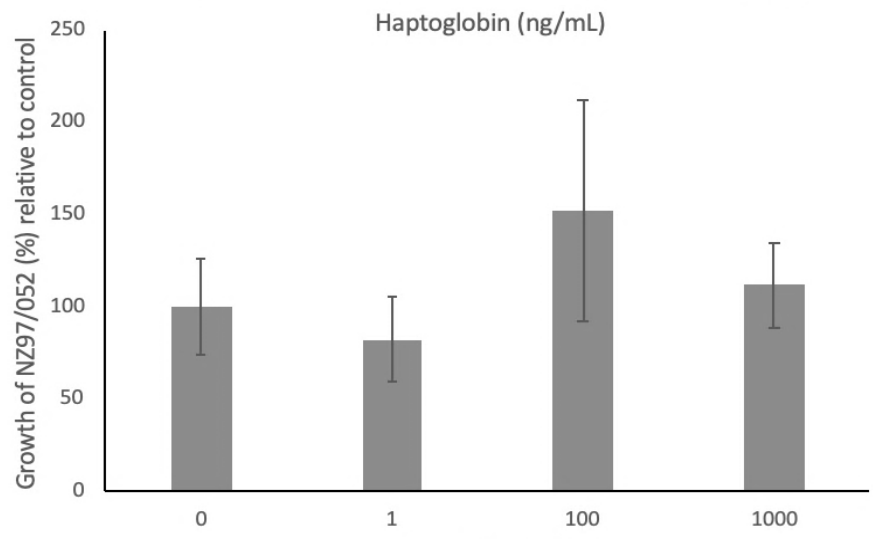

C

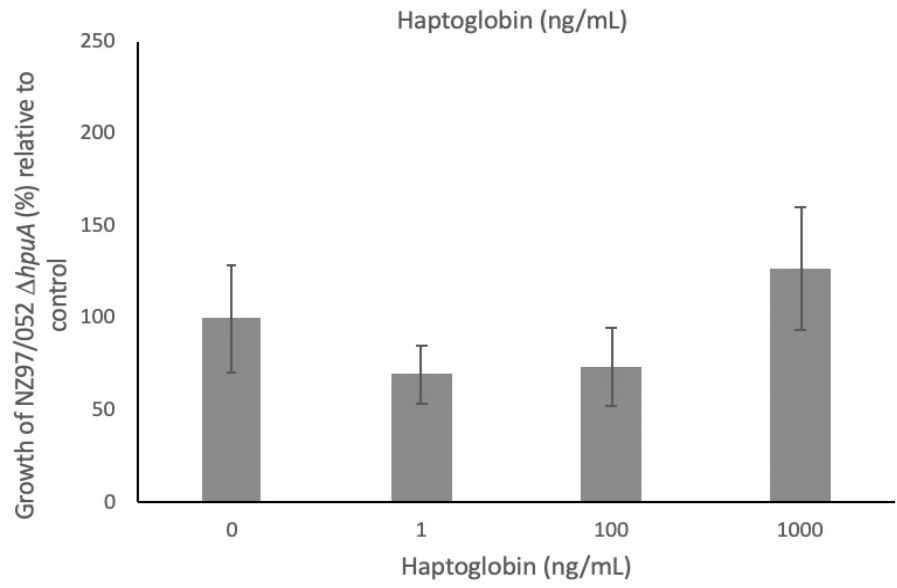

Figure 6.4. Purified human haptoglobin does not exhibit any antimicrobial effects on meningococcal isolates.

Purified haptoglobin (Sigma-Aldrich, Missouri USA) was added to 16HBE cells co-cultured with meningococci. After 24 hours, the number of meningococcal isolates were enumerated and compared with the number of viable bacteria at time zero (prior to addition of haptoglobin). Growth of each isolate with haptoglobin was normalised to their growth without added haptoglobin. The growth of meningococcal carriage isolate NZCM112 (A), disease 
isolate NZ97/052 (B) and isogenic mutant NZ97/052 $\triangle h p u A(C)$ were evaluated in the presence of 16HBE cells and haptoglobin added at the indicated final concentrations. The experiment was performed in triplicate with three technical replicates.

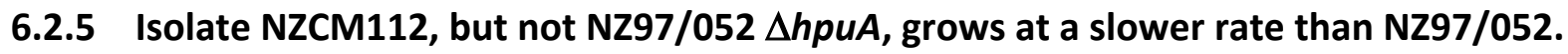

Bacterial growth assays were carried out for NZ97/052, NZCM112, and NZ97/052 $\Delta h p u A$ isolates to determine whether deletion of $h p u A$ has any significant impact on bacterial growth. Absorbance was measured over a time course to determine bacterial growth in the absence of co-cultured host cells (Figure 6.5). Overnight meningococcal cultures were resuspended in M199 medium with $10 \%$ FBS to reach an initial optical density at $600 \mathrm{~nm}\left(\mathrm{OD}_{600}\right)$ of 0.2 . Bacterial cultures were incubated at $37^{\circ} \mathrm{C}$, without shaking, to avoid the formation of aerosols. The liquid cultures were grown in a T-75 flask placed flat in the incubator to maximise oxygen availability. The $\mathrm{OD}_{600}$ was measured every two hours, until stationary phase was reached.

Optical density measurements were plotted on a semi-logarithmic scale and the bacterial growth rate was determined for the period of time during which exponential growth occurred, from two to six hours (Figure 6.5). The specific growth rate (k) was calculated for each isolate using the following equation:

$$
\mathrm{k}=2.303\left(\log N-\log N_{0}\right) / t
$$

where $t$ represents time elapsed (in hours), and where $N$ and $N_{0}$ are the $O D_{600}$ readings (assumed to be proportional to the numbers of cells) at the final and initial time points respectively.

Specific growth rate was calculated for multiple cultures and then averaged $(n=6)$. The average specific growth rate of the NZ97/052 isolate was $0.189 \mathrm{hr}^{-1}$ and the average growth rate of NZCM112 was much lower, at $0.122 \mathrm{hr}^{-1}$. The average growth rate of NZ97/052 $\triangle$ hpuA was $0.187 \mathrm{hr}^{-1}$, similar to that of the NZ97/052 isolate $\left(0.189 \mathrm{hr}^{-1}\right)$. The final $\mathrm{OD}_{600}$ reached by the isolates was also compared. After 26 hours, NZCM112 reached an $\mathrm{OD}_{600}$ of 0.439 , a significantly lower value than that of the NZ97/052 isolate, which reached a final $O_{600}$ of $0.842(p<0.001)$. 
A small difference was also seen for the final $\mathrm{OD}_{600}$ of NZ97/052 $\Delta h p u A$ isolate (0.773) compared to the NZ97/052 isolate (0.842), but this did not reach significance $(p=0.08)$. Therefore, the NZCM112 isolate grew much more slowly, and also reached stationary phase at a lower cell density, relative to NZ97/052.

These results confirm the difference in growth rate between the carriage-associated isolate, NZCM112, and disease-associated isolate, NZ97/052, which was previously shown (Figure 4.4). The results reveal that the growth rates of the wild-type NZ97/052 isolate and the NZ97/052 hpuA mutant are not substantially different, although the hpuA mutant did not reach as high a final concentration of bacteria. In all cases, stationary phase was reached at about the same time. It is also apparent that deletion of hpuA only partially accounts for the difference in growth between the NZ97/052 and NZCM112 isolates. Therefore, it is likely there are additional variants between these two isolates that contribute to the slower growth rate of NZCM112.

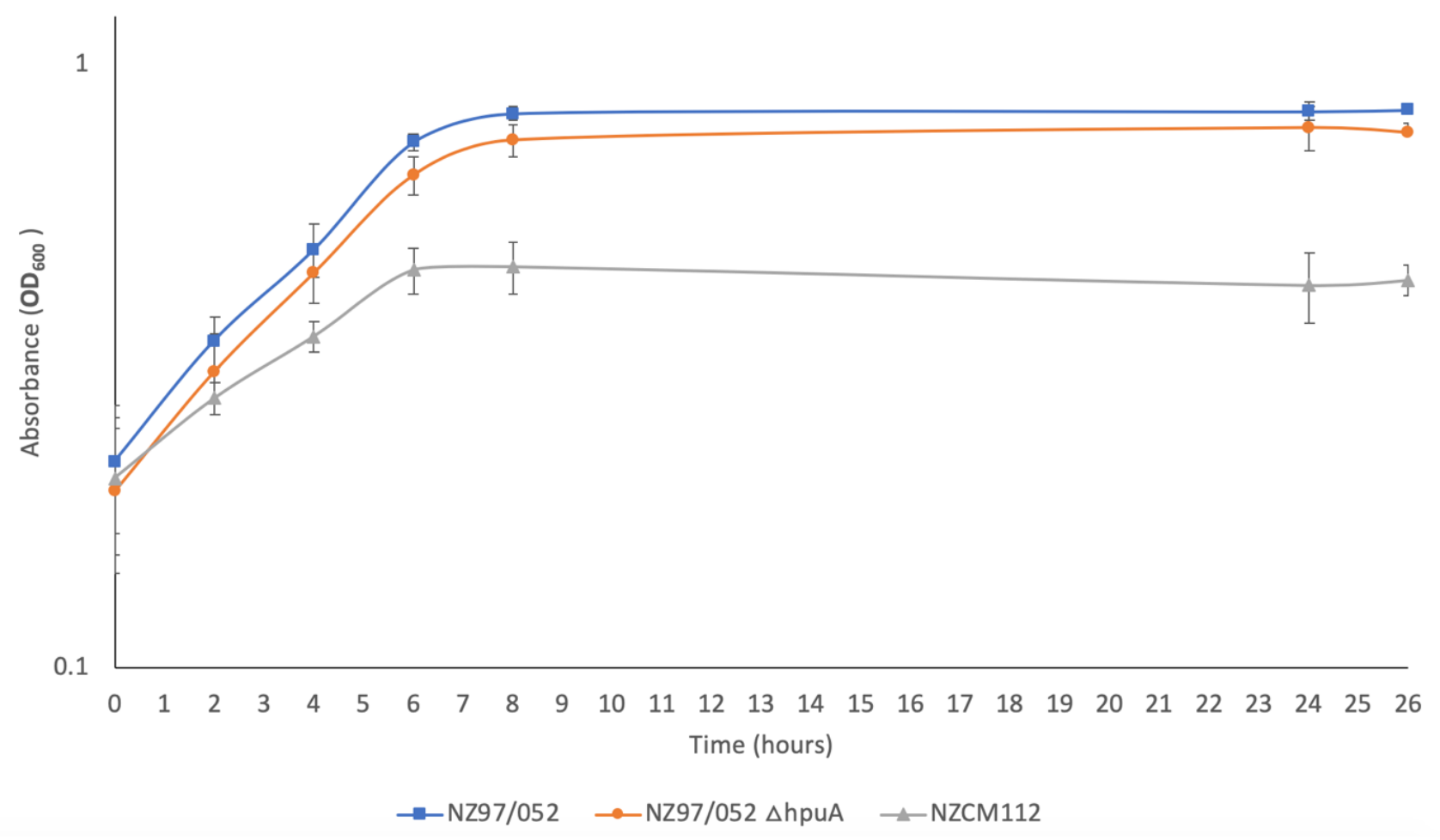

Figure 6.5. NZCM112 has a reduced growth rate compared with NZ97/052 and NZ97/052 $\triangle$ hpuA in standing liquid culture over a time course. The isolates, NZ97/052 (blue), NZCM112 (grey), and NZ97/052 $\Delta h p u A$ (orange) were grown in M199 medium with 10\% FBS (without any $16 \mathrm{HBE}$ cells), in standing liquid cultures. The $\mathrm{OD}_{600}$ was 
measured and plotted against time (hours) on a semi-logarithmic scale. The experiment was performed twice with three technical replicates for each condition. Error bars represent standard deviation.

\subsubsection{NZCM112 has reduced growth in the presence of respiratory epithelial cells, relative to $N Z 97 / 052$ and NZ97/052 $\triangle$ hpuA}

Absorbance growth curves may be used to determine bacterial growth over time, but do not distinguish between viable and non-viable bacteria. HpuA plays a role in the acquisition of iron during epithelial cell co-culture, therefore, the number of viable bacteria after an extended incubation with host cells was assessed. In order to measure bacterial growth under similar conditions as those of the respiratory epithelial cell migration assays, the numbers of viable bacteria were determined by colony enumeration.

16HBE cells were grown to confluent monolayers in 24-well plates, and overnight cultures of NZ97/052, NZCM112, and NZ97/052 $\triangle$ hpuA isolates grown on CBA plates were used to infect these cells. This growth assay was performed in M199 medium with 10\% FBS to replicate conditions of the cell migration assays. Bacterial growth was calculated by enumerating CFUs after 16 hours of bacteria and host cell co-culture. This assay compared CFU after 16 hours to input CFU, normalised relative to the growth of the NZ97/052 isolate.

Results from this assay revealed a significantly higher level $(p=0.032)$ of bacterial growth in NZ97/052 vs NZCM112; growth of NZCM112 was 25.56\% that of NZ97/052 (Figure 6.6). This was an approximately 3.9 fold difference in growth between isolates, and reinforced results reported in Chapter Three (Figure 3.4). There was also a significant difference $(p=0.011)$ in the total growth of NZ97/052 $\triangle h p u A$ and the NZCM112 isolate, relative to NZ97/052 (88.56\% vs $25.56 \%$ growth of NZ97/052). However there was not a statistically significant difference in growth between the NZ97/052 and NZ97/052 $\triangle$ hpuA isolates ( $p=0.705)$.

The number of bacterial generations of each isolate were also calculated using the following equation, where $n$ is the number of generations, $\mathrm{N}$ is the final number of cells, and $\mathrm{N}_{0}$ is the initial number of cells: 


$$
n=3.3\left(\log N-\log N_{0}\right)
$$

The number of bacterial generations was 8.6 for NZ97/052, 8.5 for NZ97/052 $\triangle$ hpuA, and 6.5 for NZCM112. These results confirm slower growth for NZCM112, which cannot be explained by deletion of hpuA. Reduced growth rate and halting bacterial growth at a lower cell density can both contribute to fewer bacteria generations.

In contrast with the absorbance assays, viable bacterial CFU were enumerated for only two time points, at zero and 16 hours. Therefore, the growth rate could not be calculated, as the period of exponential growth could not be determined for this assay.

The deletion of hpuA from NZ97/052 did not result in significant differences in the specific growth rate during exponential phase (Figure 6.5), or the final cell density reached during stationary phase. Therefore, although the growth assay measuring absorbance showed a slightly lower final concentration of bacteria reached for NZ97/052 $\triangle h p u A$, it does not appear that the hpuA deletion has a detrimental effect on growth of $N$. meningitidis. It is unlikely that the reduced ability of NZ97/052 $\triangle h p u A$ to inhibit wound repair (Figure 5.13), or the reduced numbers of adherent bacteria (Figure 6.3), is due to impaired growth or lower numbers of bacterial cells. 


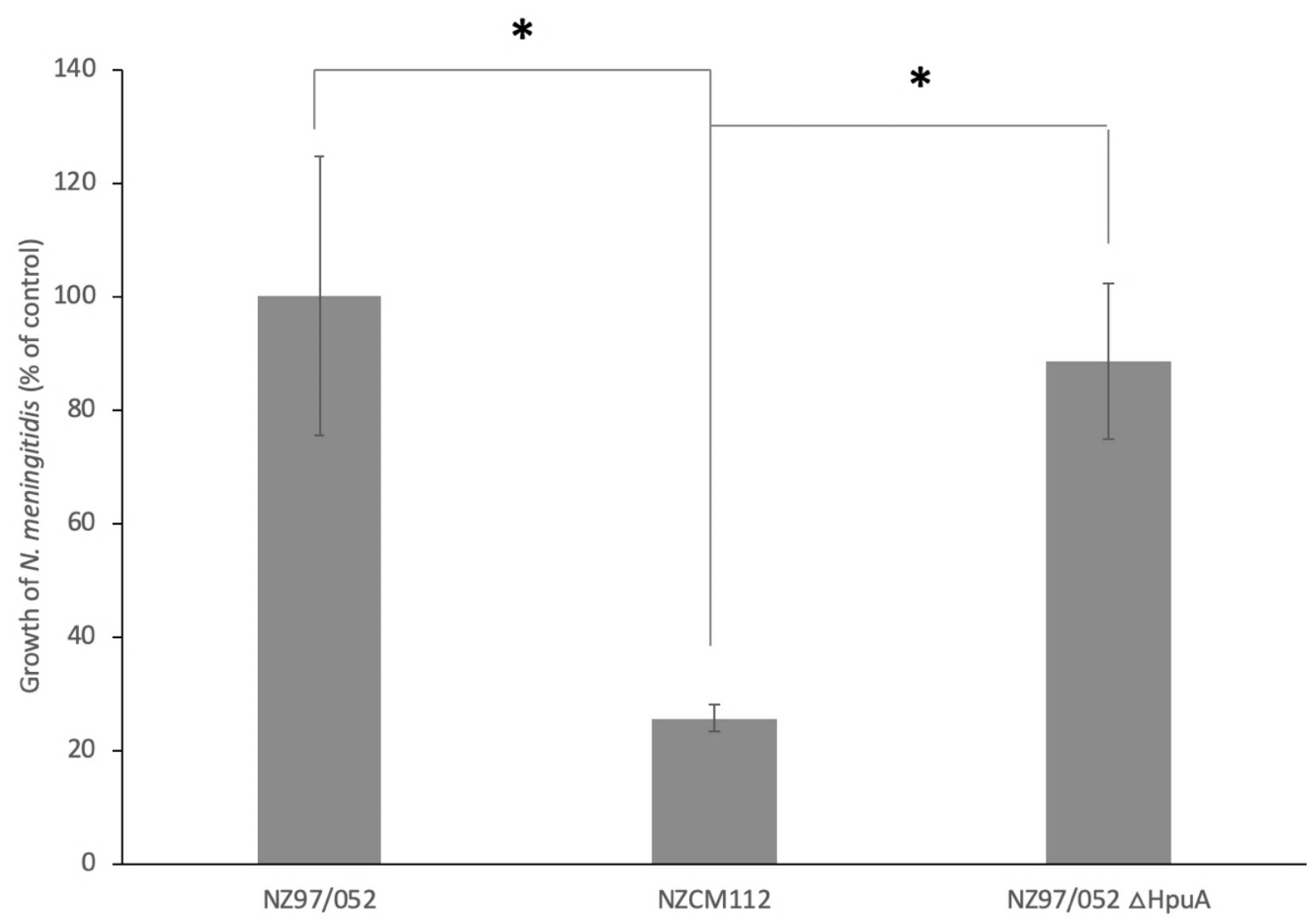

Figure 6.6. Growth of NZCM112 is lower than NZ97/052 and NZ97/052 $\triangle$ hpuA in the presence of $16 \mathrm{HBE}$ cells. Assay comparing CFU at 16 hours to input CFU, normalised relative to the growth of NZ97/052 isolate. The experiment was performed three independent times with three technical replicates. ${ }^{*}$ Represents $p$ value $<0.05$.

\subsubsection{HpuA increases association of bacteria with human respiratory epithelial cells.}

Fluorescence microscopy used to assess levels of intracellular haptoglobin revealed greatly reduced numbers of NZMC112 and NZ97/052 $\triangle h p u A$ adherent to epithelial cells, relative to NZ97/052 bacteria. This is not likely due to reduced growth rate or enhanced susceptibility to the antimicrobial effects of haptoglobin for NZ97/052 $\triangle h p u A$, which had a similar growth rate and haptoglobin resistance as the wild-type parent (Figure 6.4, Figure 6.6). Experiments described in Chapter Three (Figure 3.6) demonstrated that NZ97/052 and NZCM112 had differing levels of association with $16 \mathrm{HBE}$ cells. 
Cell association assays enable quantification of all viable bacteria that are intracellular or tightly adhered to the surface of host cells. To determine whether HpuA influences this phenotype, the association of the NZ97/052 $\triangle h p u A$ isolate to $16 \mathrm{HBE}$ cells was compared to the wild-type parent. Mid-log phase N. meningitidis were co-cultured with $16 \mathrm{HBE}$ cells for one hour. Cells were then washed thoroughly to remove all non-adherent bacteria, and saponin was used to lyse $16 \mathrm{HBE}$ cells without affecting the viability of the meningococci. Enumeration of total cellassociated bacteria (adherent and intracellular) was determined by plating and counting CFU. Cell association of the NZCM112, NZ97/052, and NZ97/052 $\triangle$ hpuA isolates was calculated as a percentage of initial inoculant, also measured by CFU enumeration (Figure 6.7A). The NZ97/052 isolate displayed about 4.6-fold higher levels of association with 16HBE cells relative to NZCM112 ( $p$ 0.001). The NZ97/052 $\Delta h p u A$ isolate had significantly decreased association with 16HBE cells relative to wild-type NZ97/052 (8.51\% and $16.05 \%$ of the initial inoculant, respectively, $\mathrm{p}<0.01$ ). Association of NZ97/052 with 16HBE cells was approximately 1.9-fold higher relative to NZ97/052 $\triangle h p u A$.

Isolates were supplemented with $20 \mu \mathrm{M} \mathrm{FeSO}_{4}$ to determine if iron caused any significant effect on cell-association (Figure 6.7B). The results support findings reported in Chapter Four (Figure 4.6); iron sulfate supplementation significantly increased mean cell association of NZCM112 from $1.5 \%$ to $3.9 \%$ ( $p<0.001)$. There was no significant effect of iron on association of $\mathrm{NZ97/052}$ isolate to $16 \mathrm{HBE}$ cells $(\mathrm{p}=0.741)$. Interestingly, $20 \mu \mathrm{M} \mathrm{FeSO}$ s supplementation caused a significant increase in association of NZ97/052 $\Delta h p u A$ to respiratory epithelial cells from $4.64 \%$ to $8.11 \%(p<0.001)$.

These cell association results are consistent with those seen in cell migration assays, suggesting that HpuA could be affecting cell wound repair through its role as an adhesin, rather than host cells being starved of haptoglobin. This also explains the reduced numbers of adherent bacteria seen in the fluorescence microscopy image (Figure 6.3) for both NZCM112 and NZ97/052 $\triangle h p u A$. This is the first indication, to our knowledge, that HpuA could function as an adhesin to epithelial cells. 
A

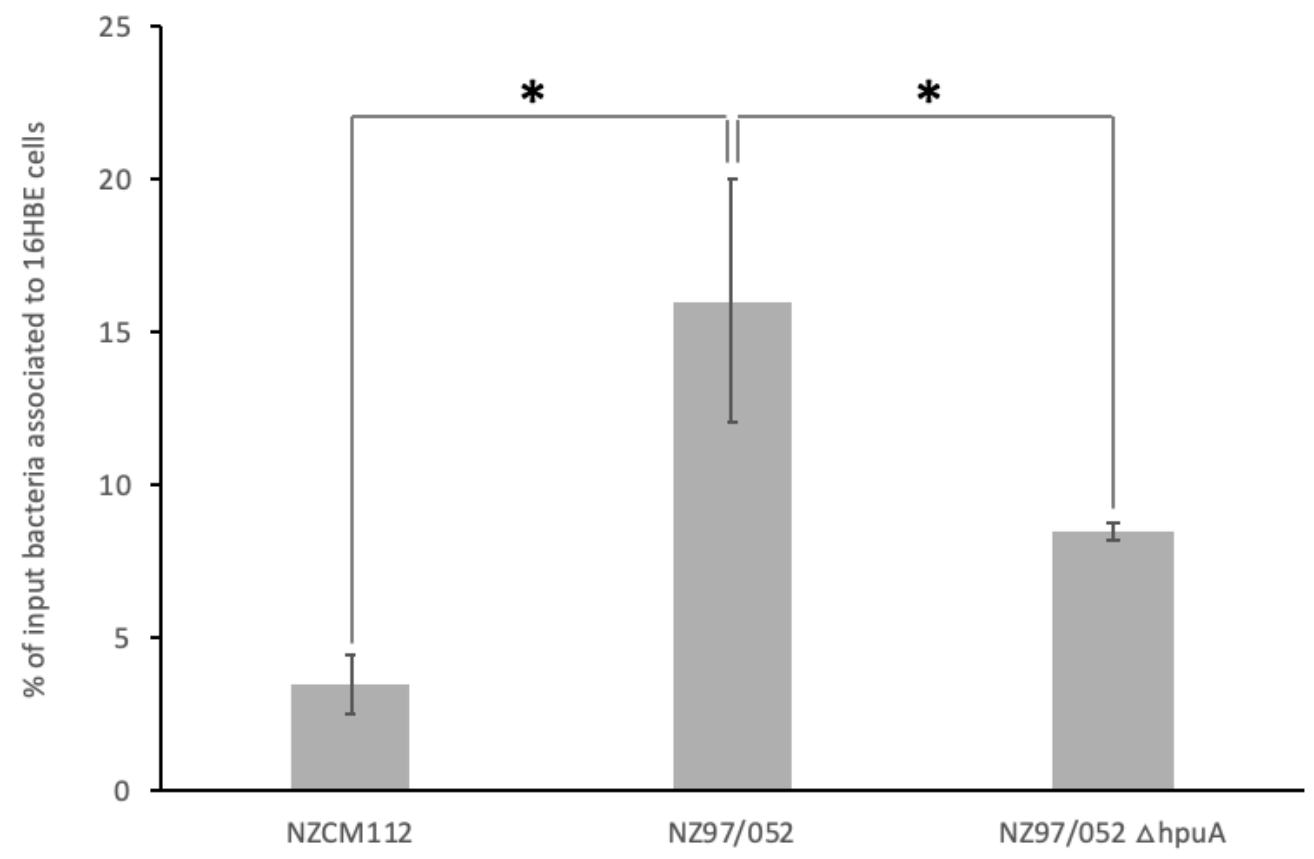

B

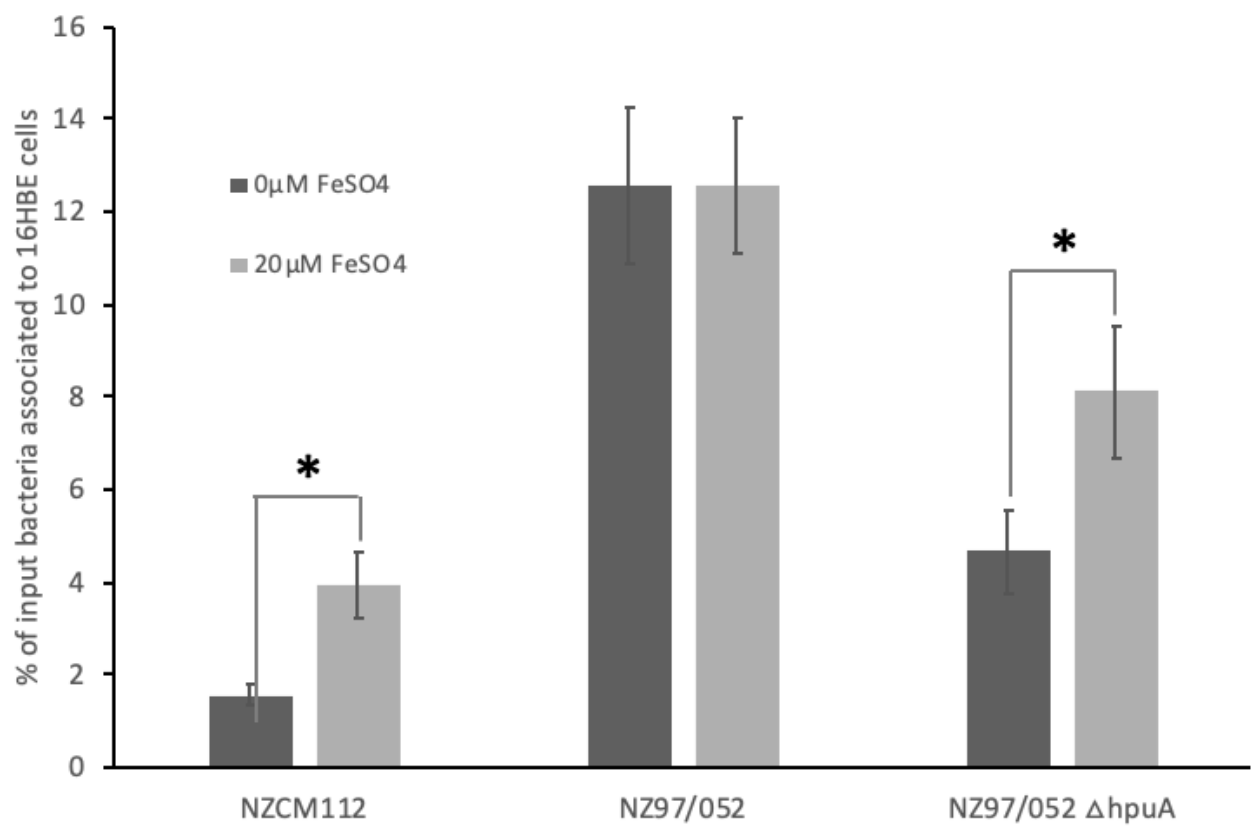

Figure 6.7. Both NZCM112 and NZ97/052 $\triangle$ hpuA are associated with $16 \mathrm{HBE}$ cells at lower rates. (A) NZ97/052 had a significantly higher association with $16 \mathrm{HBE}$ cells than NZCM112 and NZ97/052 $\Delta h p u A$. (B) Supplementation of bacteria-cell co-culture with $20 \mu \mathrm{M}$ of $\mathrm{FeSO}_{4}$ caused a significant increase in cell association for NZCM112 and NZ97/052 $\triangle$ hpuA isolates, but not the NZ97/052 wild-type. Experiments were performed in triplicate, three independent times. Error bars represent standard deviation. ${ }^{*}$ Represents $p$-value $<0.05$. 


\subsubsection{NZ97/052 displays higher levels of invasion of human respiratory epithelial cells than NZCM112 and NZ97/052 $\Delta$ hpuA}

Host cell invasion assays were completed to determine whether the increased bacteria association was a result of increased cellular invasion. A gentamicin protection assay was used to quantify bacterial invasion into $16 \mathrm{HBE}$ cells. This assay measured the intracellular NZCM112, NZ97/052, and NZ97/052 $\triangle h p u A$ bacteria as a percentage of the initial inoculum, following a four hour incubation (Figure 6.8A). The NZ97/052 isolate displayed significantly higher levels of invasion of $16 \mathrm{HBE}$ cells than NZCM112 ( $<<0.001)$, an approximately 2.3 fold increase in intracellular bacteria. The NZ97/052 isolate had an approximately 1.7 fold higher rate of invasion into $16 \mathrm{HBE}$ cells, relative to NZ97/052 $\triangle h p u A$, a significant difference $(\mathrm{p}=0.029)$.

A host cell invasion assay was also carried out in the presence of $20 \mu \mathrm{M} \mathrm{FeSO}_{4}$ to determine whether iron had an effect on cell invasion by the isolates (Figure 6.8B). Results seen were very similar to those seen with the cell association assay; no significant difference was observed in the invasion rate of NZ97/052 cells with or without iron sulfate supplementation $(0.46 \%$ and 0.49\% respectively). However, supplementation with $20 \mu \mathrm{M} \mathrm{FeSO}$ significantly increased invasion by carriage-associated isolate NZCM112 from $0.09 \%$ to $0.22 \%(p<0.001)$. This effect was also seen with the NZ97/052 $\triangle$ hpuA isolate; supplementation with iron increased host cell invasion from $0.11 \%$ to $0.27 \%(p=0.039)$.

The gentamicin protection assay results were consistent with the results seen with the cell association assay (Figure 6.7). The levels of intracellular bacteria accounted for a tiny fraction of

cell associated bacteria, therefore, increased rates of cell invasion can be explained by increased levels of cell association. Adherence of extracellular bacteria is likely driving both the numbers of cell associated bacteria and the rates of invasion. 
A
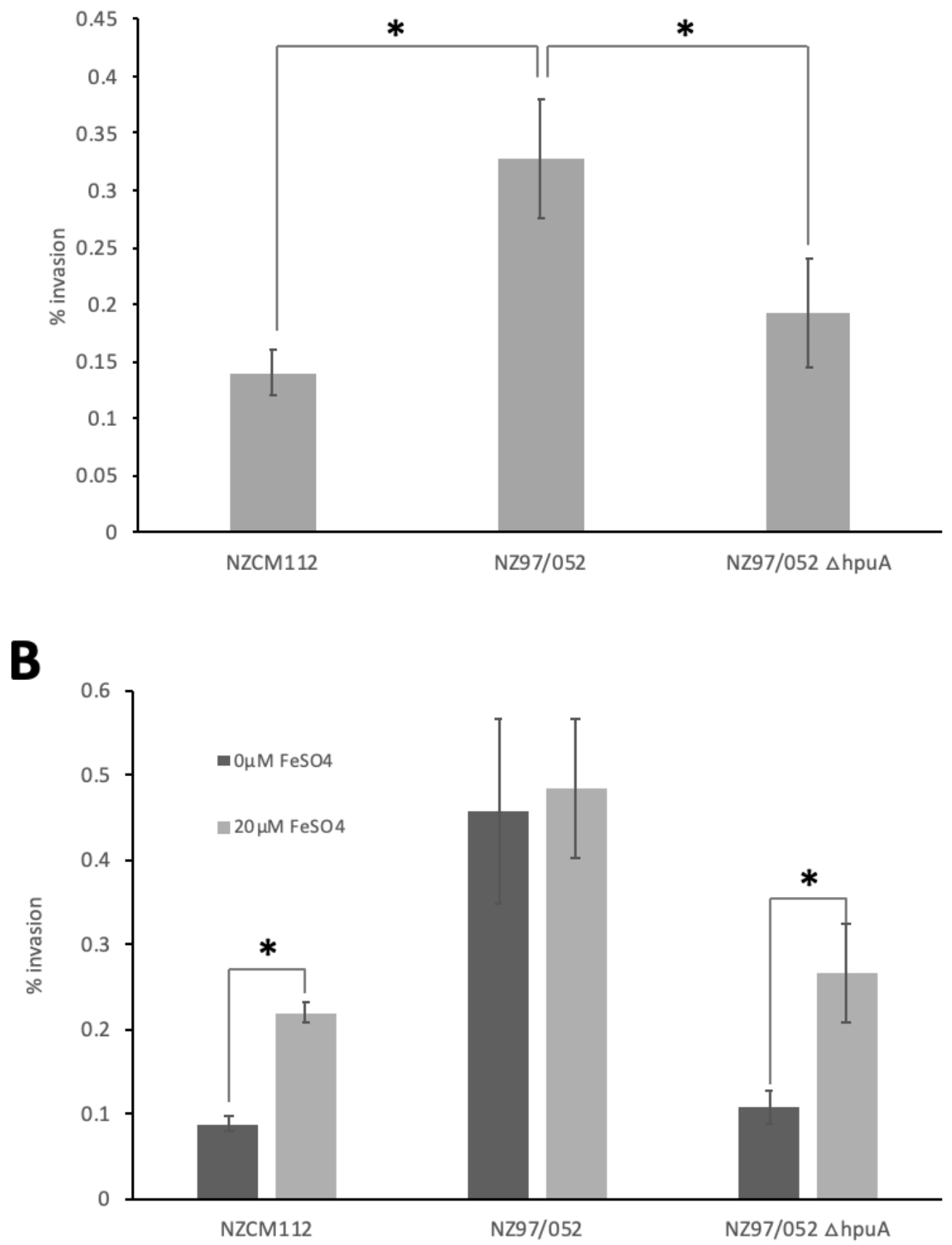

Figure 6.8. NZ97/052 invades epithelial cells at a higher rate than NZCM112 or NZ97/052 $\Delta$ hpuA. The number of bacteria recovered after gentamicin treatment and saponin lysis is presented as a percent of the inoculum. (A) NZ97/052 had a significantly higher rate of invasion of 16HBE epithelial cells than NZCM112 and NZ97/052 $\Delta h p u A$. (B) Supplementation of bacteria-cell co-culture with $20 \mu \mathrm{M} \mathrm{FeSO}_{4}$ caused a significant increase in cell invasion for the NZCM112 and NZ97/052 $\triangle h p u A$ isolates, but not for NZ97/052. Experiments were performed in triplicate, three independent times. Error bars represent standard deviation. * Represents $p$-value $<0.05$ 


\subsubsection{Fluorescence microscopy analysis confirms reduced bacterial attachment of NZCM112 and NZ97/052 $\triangle$ hpuA to $16 \mathrm{HBE}$ cells}

To confirm the differences in adhesion between isolates to $16 \mathrm{HBE}$ cells, fluorescence microscopy was carried out. Slides were prepared and fluorescent staining was completed as described in Section 6.2.3, but with slides mounted and examined immediately following staining with DAPI. These findings strengthen results from the association assay; the NZ97/052 isolate had much higher association with 16HBE cells than NZCM112 or NZ97/052 $\Delta$ hpuA (Figure 6.9).

These results support the theory that HpuA plays a role in the association of disease-associated NZ97/052 isolate to host epithelial cells. There was also an interesting pattern of adherence of NZ97/052 to the host cells. It appeared that NZ97/052 formed many microcolonies on the surface of the host cells, that were not seen with the NZCM112 or NZ97/052 $\triangle$ hpuA isolates. This adherence pattern could be the subject of future research to help identify the cellular target of HpuA. 

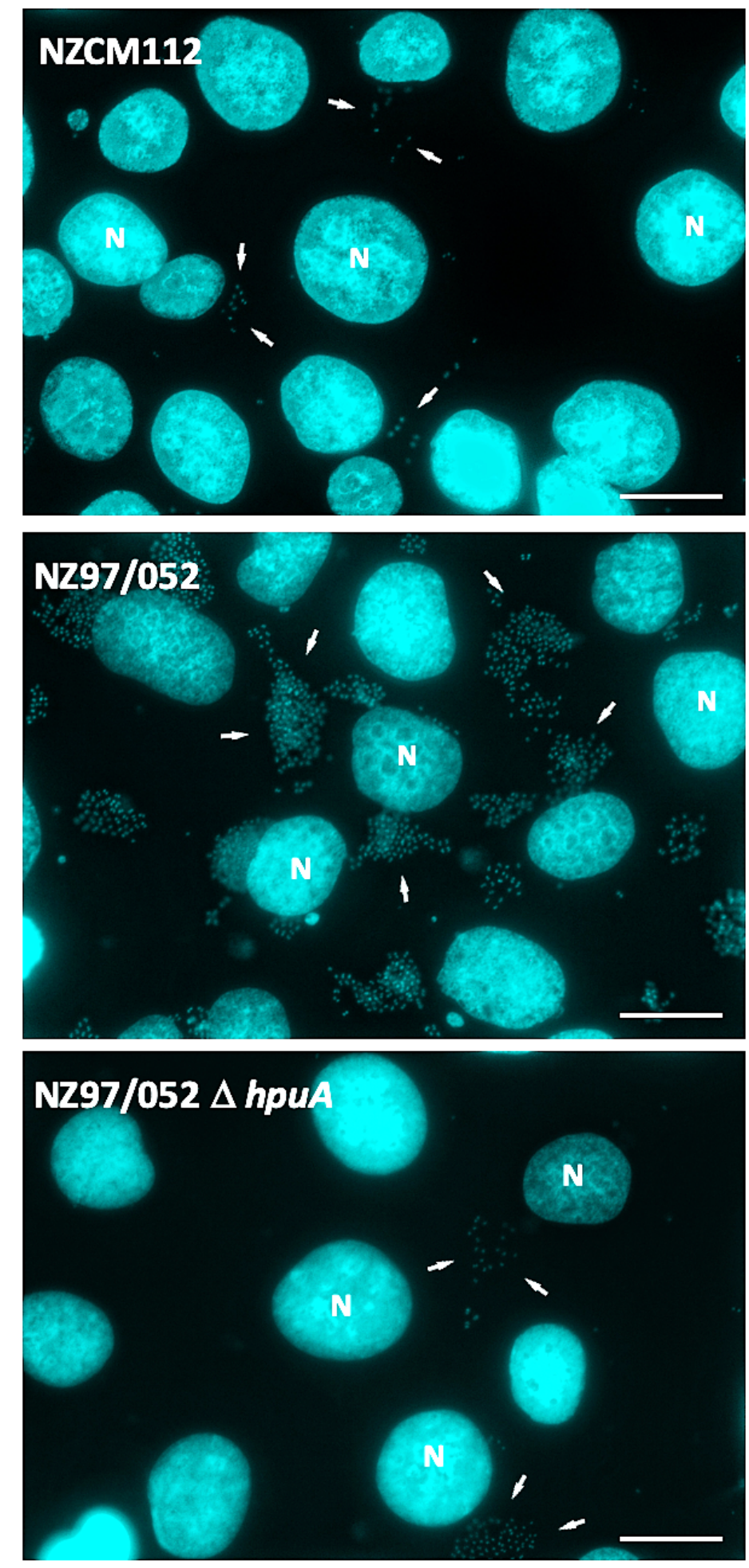

Figure 6.9. Fluorescence microscopy showing association of NZCM112, NZ97/052, and NZ97/052 $\Delta$ hpuA isolates with 16HBE cells. DAPI stained bacteria nuclei are indicated by white arrows. 16HBE cell nuclei are indicated by N. Scale bar, $20 \mu \mathrm{m}$. 


\subsubsection{Heterologous expression of HpuA in E. coli}

The expression of HpuA was shown to be important in mediating adherence in an NZ97/052 wild-type background (Figure 6.7, Figure 6.9). The HpuA protein is a lipoprotein that acts in concert with its outer membrane protein partner, $\mathrm{HpuB}$, to harvest iron from haemoglobin. Because $h p u A$ and $h p u B$ are co-ordinately regulated and co-transcribed in an operon, previous studies have shown that mutations in $h p u A$ result in polar loss of $h p u B$ transcription (Chen et al., 1998). The transcriptome analysis (Chapter Five, Section 5.2.2) revealed minimal hpuB transcription in NZCM112. Therefore, the possibility that the phenotypes described for the NZ97/052 $\triangle h p u A$ may be in part due to loss of expression of HpuB could not be ruled out. Because HpuB is localised in the outer membrane, it could also play a role in adherence to epithelial cells. There were technical difficulties, including a lack of replicating plasmids, which prevented complementing the $\triangle h p u A$ mutant in NZ97/052.

An alternative approach was taken to investigate if $\mathrm{HpuA}$ is sufficient by itself to mediate adherence. Using an inducible plasmid, hpuA was heterologously expressed in E. coli. An E. coli codon-optimised hpuA gene fragment was synthesised and cloned into the pET28a(+) expression vector (Novagen) by Twist Biosciences. The resulting plasmid was introduced into HiControl BL21 E. coli (Lucigen). Although codon optimisation resulted in the nucleotide sequence of the gene that was changed significantly from the original NMBG2136 meningococcal sequence of hpuA, the resulting amino acid sequence was identical (Appendix, Section 9.5). This approach also enabled the removal of the slipped-strand mispairing error-prone phase variable homopolymeric G-tract, a run of 14 guanine bases that can result in frameshift errors during PCR or plasmid replication in E. coli. Retaining an identical amino acid sequence, without phase variable fragments, reduced the risk of errors. Codon optimisation was also used to enhance the amount of protein produced, after it was cloned, as differing codon usage between $E$. coli and $N$. meningitidis may have inhibited robust protein expression.

Expression of HpuA by the PET28a expression vector is under control of the T7 lac promoter, and can be induced by IPTG or repressed by glucose. To confirm the expression of HpuA by 
transformed BL21 E. coli, expression was either induced with 0.4 mM IPTG or repressed with $1 \%$ glucose for one hour at $37^{\circ} \mathrm{C}$. Total cell protein fractions were analysed by sodium dodecyl sulfate polyacrylamide gel electrophoresis (SDS-PAGE) on a Novex 10-20\% Tris-Glycine mini wedge well gel (Life Technologies, Auckland). Coomassie blue stain was used to visualise the protein gel.

The predicted protein size of HpuA, based on amino acid sequence, is $\sim 35 \mathrm{kDa}$. SDS-PAGE revealed successful expression of HpuA by induced, transformed BL21 E. coli (Figure 6.10). The level of expression of HpuA was slightly higher after three hours of induction, relative to one hour, but both resulted in detectable protein. E. coli cultures that were repressed with $1 \%$ glucose for three hours did not appear to express detectable HpuA (Figure 6.10). These results show successful transformation of $E$. coli, which express HpuA only with addition of IPTG. This strain was next used for growth and cell association assays. 
$198 \mathrm{kDa}$

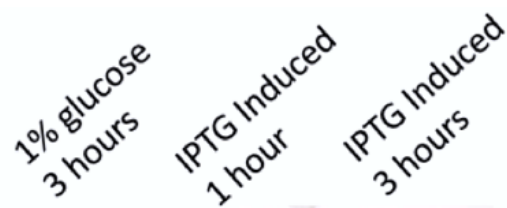

$98 \mathrm{kDa}$

$62 \mathrm{kDa}$

$49 \mathrm{kDa}$

$38 \mathrm{kDa}$

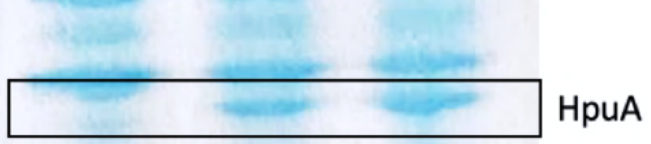

$28 \mathrm{kDa}$

$17 \mathrm{kDa}$

Figure 6.10. SDS-PAGE gel of total cell protein fraction for $E$. coli induced with $0.4 \mathrm{mM}$ IPTG or repressed with $1 \%$ glucose for one hour or three hours. Protein sizes were determined using the SeeBlue Plus2 Pre-Stained protein standard (Thermo Fisher Scientific, USA). The black box outlines HpuA expression. The predicted protein size of $\mathrm{HpuA}$ is $\sim 35 \mathrm{kDa}$. Cell fractions induced with IPTG for one hour or three hours were collected from independent culture batches.

\subsubsection{Expression of HpuA by E. coli did not adversely impact bacterial growth}

Before $E$. coli was used to measure the impact of HpuA on cell association, the viability of bacteria expressing HpuA was tested. Protein overexpression often impacts bacterial fitness and may slow growth. Growth rate analysis was performed on IPTG-induced and glucose-repressed cultures of the BL21 E. coli strain with the hpuA gene cloned into the pET28a vector. The goal of this experiment was to determine whether protein overexpression adversely impacted growth 
of $E$. coli and to establish the most appropriate time of IPTG induction of $E$. coli before infecting epithelial cells.

Overnight cultures of BL21 Hi-Control E. coli with the hpuA-expression plasmid were diluted in LB broth with $50 \mu \mathrm{g} / \mathrm{mL}$ kanamycin to a starting $\mathrm{OD}_{600}$ of 0.04 . E. coli was grown under two conditions, with either $0.4 \mathrm{mM}$ IPTG or $1 \%$ glucose (final concentration of $10 \mathrm{~g} / \mathrm{L}$ ), to induce or repress expression of the $h p u A$ gene respectively. Absorbance measurements were taken over a time course, and used to calculate bacterial growth (Figure 6.11). $O D_{600}$ measurements were plotted on a semi logarithmic scale, and bacterial growth was determined for the time period where growth was exponential. The specific growth rate $(k)$ was calculated for each isolate using the following equation:

$$
\mathrm{k}=2.303\left(\log N-\log N_{0}\right) / t
$$

where $t$ represents time elapsed (in hours), and where $N$ and $N_{0}$ are the $O D_{600}$ readings (assumed to be proportional to the numbers of cells) at the final and initial time points respectively.

Between 30 and 60 minutes the growth rate of uninduced cultures was $3.848 \mathrm{hr}^{-1}$ and the growth rate of cultures induced with $0.4 \mathrm{mM}$ IPTG was $3.629 \mathrm{hr}^{-1}$. After 90 minutes, the growth rate of $E$. coli induced with IPTG slowed (Figure 6.11), however, the final $\mathrm{OD}_{600}$ of the bacterial cultures at 24 hours did not significantly differ between BL21 E. coli regardless of expression of hpuA $(\mathrm{p}=0.185)$. The results from this growth assay suggest BL21 E. coli induced to overexpress hpuA did exhibit a slightly reduced growth rate, however, this reduction was minor. This difference in growth was not entirely unexpected, as overexpression of proteins can often result in decreased bacterial fitness, even if there is no toxic activity of the proteins (Klumpp \& Hwa, 2014; Price et al., 2016). That the inducing strain reached a high OD overnight suggested that there was minimal toxicity in the expression of HpuA for the host $E$. coli strain. 


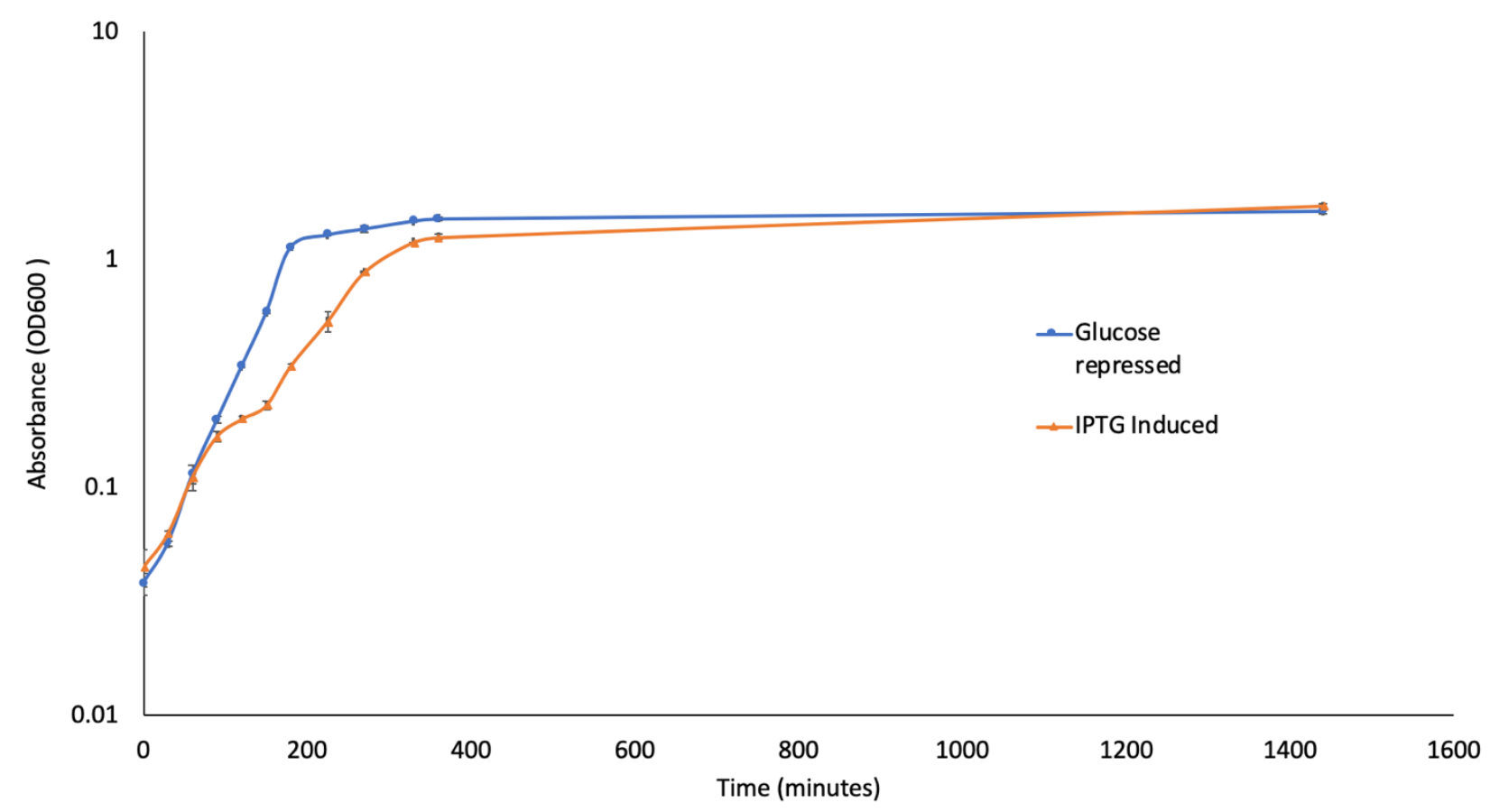

Figure 6.11. Growth curve of BL21 E. coli associated to 16HBE cells. E. coli expression of HpuA was either induced with 0.4 mM IPTG (orange) or repressed with $1 \%$ glucose (blue). IPTG or glucose was added at hour zero. The OD 600 was plotted on a semi-logarithmic scale. The experiment was performed two independent times, with three technical replicates for each condition. Error bars represent standard deviation.

\subsubsection{E. coli expressing HpuA has increased association with 16HBE respiratory epithelial cells.}

Cell association assays were carried out for induced and repressed cultures of $E$. coli to investigate whether expression of HpuA facilitates bacterial adhesion to 16HBE cells. Mid-log phase cultures of $E$. coli were induced to express HpuA with IPTG or repressed with glucose for one hour, as this had resulted in sufficient expression of HpuA with no defect in $E$. coli growth (Figure 6.10, Figure 6.11). The $\mathrm{OD}_{600}$ was measured, then $E$. coli was added to confluent $16 \mathrm{HBE}$ cells at an $\mathrm{MOI}$ of approximately 10 and the co-culture was incubated for 30 minutes. Experiments completed in the laboratory previously found that $E$. coli was very damaging to $16 \mathrm{HBE}$ cells over longer incubation periods, such as those used for meningococcal cell association assays. To determine the most appropriate time for bacterial attachment to host 
cells, E. coli were co-cultured with $16 \mathrm{HBE}$ cells at an $\mathrm{MOI}$ of 10 and the integrity of $16 \mathrm{HBE}$ cells was assessed by examination under a microscope at various time points. The $16 \mathrm{HBE}$ cell monolayer was still intact at $\mathbf{3 0}$ minutes, which is why this time was selected for the $E$. coli association assay. At one hour, the time used for N. meningitidis association assays, damage to the host cells started to become evident. After incubating $16 \mathrm{HBE}$ cells with induced or repressed HpuA-expressing BL21 cells for 30 minutes, non-adherent bacteria were removed by washing thoroughly and cells were lysed with saponin. Enumeration of cell-associated E. coli was determined by plating and counting CFU. Associated bacteria were calculated as a percentage of the initial inoculant, also measured by CFU enumeration.

The average association of $E$. coli after 30 minutes was $0.40 \%$ of input bacteria for $E$. coli not expressing HpuA, and $0.83 \%$ of input bacteria that were induced to express HpuA. This was a significant difference $(p=0.008)$, and represented an approximately 2 fold difference in association with respiratory epithelial cells (Figure 6.12). This difference in association with HpuA was very similar to that described in Section 6.2.6, where association of NZ97/052 with 16HBE cells was approximately 1.9-fold higher than NZ97/052 $\triangle$ hpuA.

The half hour co-culture with bacteria and host cells was too short for numbers of bacteria to widely diverge. These results indeed suggest that HpuA is both necessary and sufficient to facilitate adhesion of bacteria to epithelial cells. Almost no intracellular $E$. coli were detected for either induced or repressed HpuA-BL21 cells, due to the short incubation period. Hence, these results are not reported here. Similarly, the period of time required for epithelial wound closure (16 hours) was too long for $16 \mathrm{HBE}$ cells to be exposed to BL21 Hi-Control E. coli expressing HpuA, as would have resulted in significant cellular damage. 


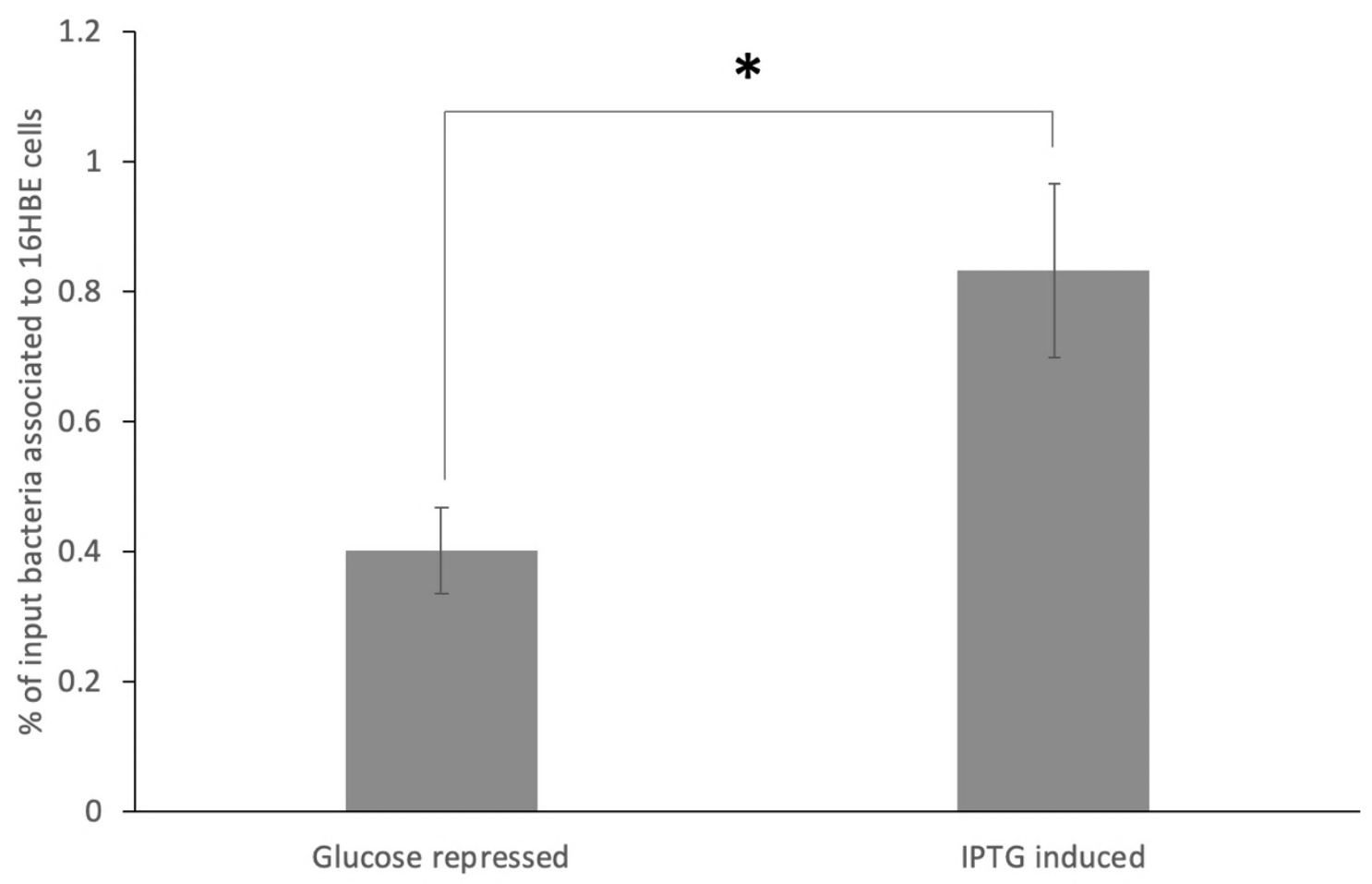

Figure 6.12. Expression of HpuA in BL21 E. coli results in increased levels of cell association with 16HBE cells. $E$. coli expression of HpuA was either induced with 0.4 mM IPTG or repressed with $1 \%$ glucose. The induced or repressed E. coli cultures were incubated with $16 \mathrm{HBE}$ cells for 30 minutes, then the cells were washed to remove non-adherent bacteria and lysed. Cell-associated bacteria were enumerated by colony counts, and were compared to numbers of input bacteria added to the cells. Experiments were performed in triplicate, three independent times. Error bars represent standard deviation. * Represents $p$-value $<0.05$.

\subsubsection{Protein sequence alignment of HpuA homologues}

Data collected suggested a role in adhesion for HpuA, a function that has not previously been described. A review of HpuA literature was carried out to search for insights into how it could play a role in adherence. A structural analysis of HpuA in the Neisseriae family was previously published by Wong et al (2015). They solved the structure and identified several loops that extend from the core of the protein; these loops are less conserved and presumably subject to antibody selection, however, several residues within these loops were shown to directly bind to 
haem. A highly conserved Cys-Cys motif in HpuA was identified adjacent to one of the loops. Mutations in this Cys-Cys motif, converting them to serines, did not affect the binding affinity of HpuA to haem, and therefore do not appear to play a role in haem binding. As this motif was completely conserved in the multiple isolates belonging to the Neisseriaceae family that were included in the study, they concluded that this motif is functional, although the function is unknown. Results from this chapter suggest that this function could be adherence to host epithelial cells. In order to further investigate this conserved Cys-Cys domain, HpuA protein alignments were completed to compare the isolates used in this study.

HpuA protein sequences from several genome-sequenced $N$. meningitidis isolates, including carriage-associated Alpha 275 (Serogroup W), and disease-associated isolates FAM18 (Serogroup C) and Z2491 (Serogroup A), were aligned. Isolates used in this study, NZ97/052 and NZCM112 (Serogroup C) were also included. All HpuA protein sequences were obtained from NCBI (Altschul et al., 1990), or from genome sequencing data (Chapter Five), and multiple sequence alignment was completed using ClustalW (Thompson et al., 1994) (Figure 6.13). Isolates were selected to represent a broad range of $N$. meningitidis isolates across different serogroups. Figure 6.13 highlights the premature stop codon in the HpuA sequence of the NZCM112 isolate. Sequence alignments demonstrated that HpuA is conserved, but with variability, even in a carriage isolate (Alpha 275). Structural loops, especially Loop 1, have some variation between isolates tested. There was also complete conservation of the Cys-Cys motif in all of the isolates aligned. 


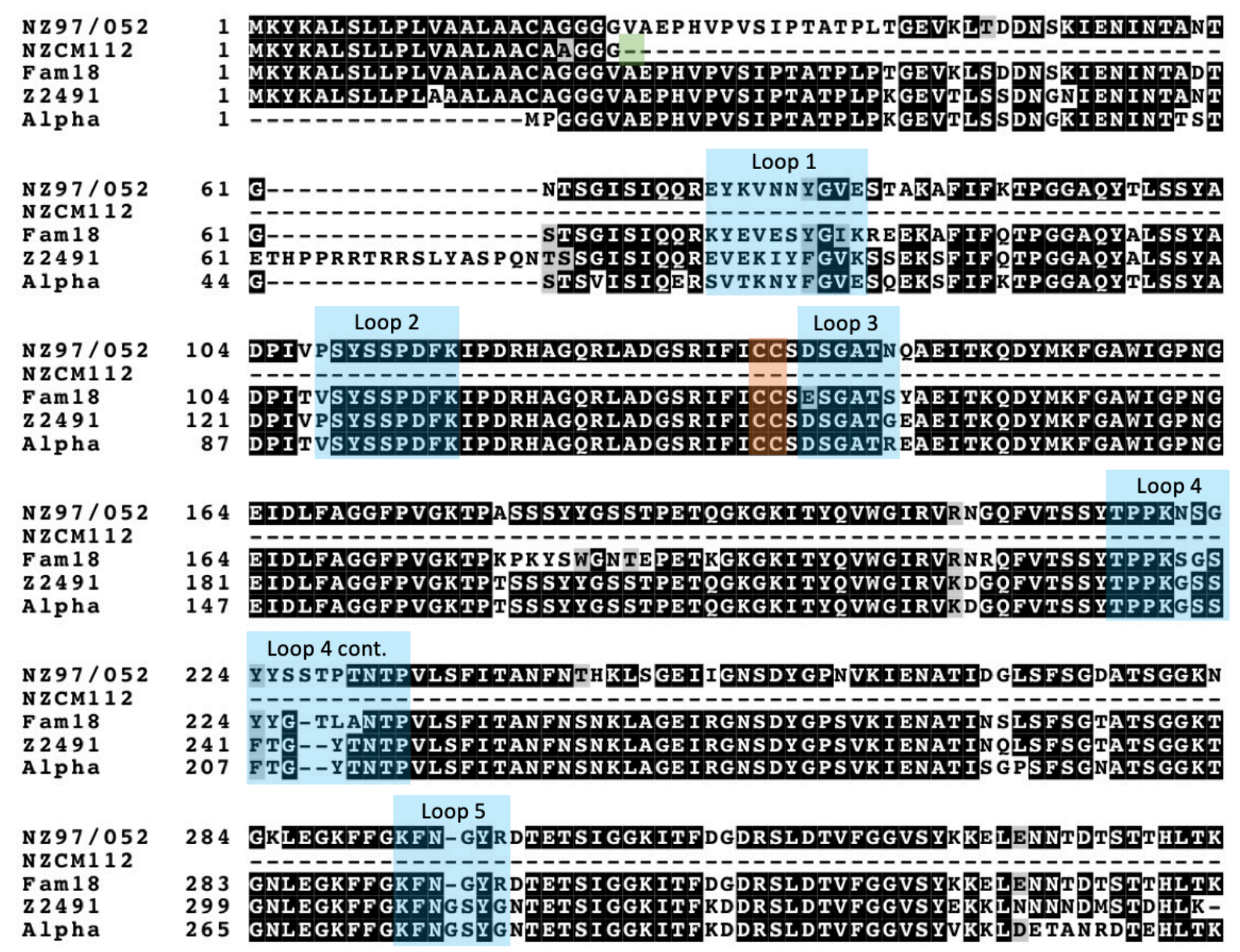

Figure 6.13. Protein sequence alignment of HpuA from $\boldsymbol{N}$. meningitidis isolates. $N$. meningitidis carriage isolate Alpha 275 (Serogroup W), N. meningitidis FAM18 (Serogroup C), N. meningitidis Z2491 (Serogroup A), N. meningitidis NZ97/052 (Serogroup C), and N. meningitidis NZCM112 (Serogroup C) were aligned using ClustalW (Thompson et al., 1994). Images were created using BOXSHADE 3.21. Black shading indicates conserved residues, grey shading indicates conservative variation. Orange shading indicates conserved Cys-Cys motif. Green shading indicates premature stop codon in NZCM112. Structural loops indicated by blue boxes.

To further investigate the regions of conservation, sequence alignment of HpuA homologues from other Neisseria species was completed. HpuA homologues were compared between $N$. meningitidis NMBG2136, N. gonorrhoeae FA 1090, and the non-pathogenic species, $N$. lactamica 020-06 (Figure 6.14). Much of the variation between HpuA homologues occurs in the structural loops, indicated by the blue boxes in Figure 6.14, a variability that may be driven by antibody pressure. The Cys-Cys motif was also completely conserved in all homologue sequences. 


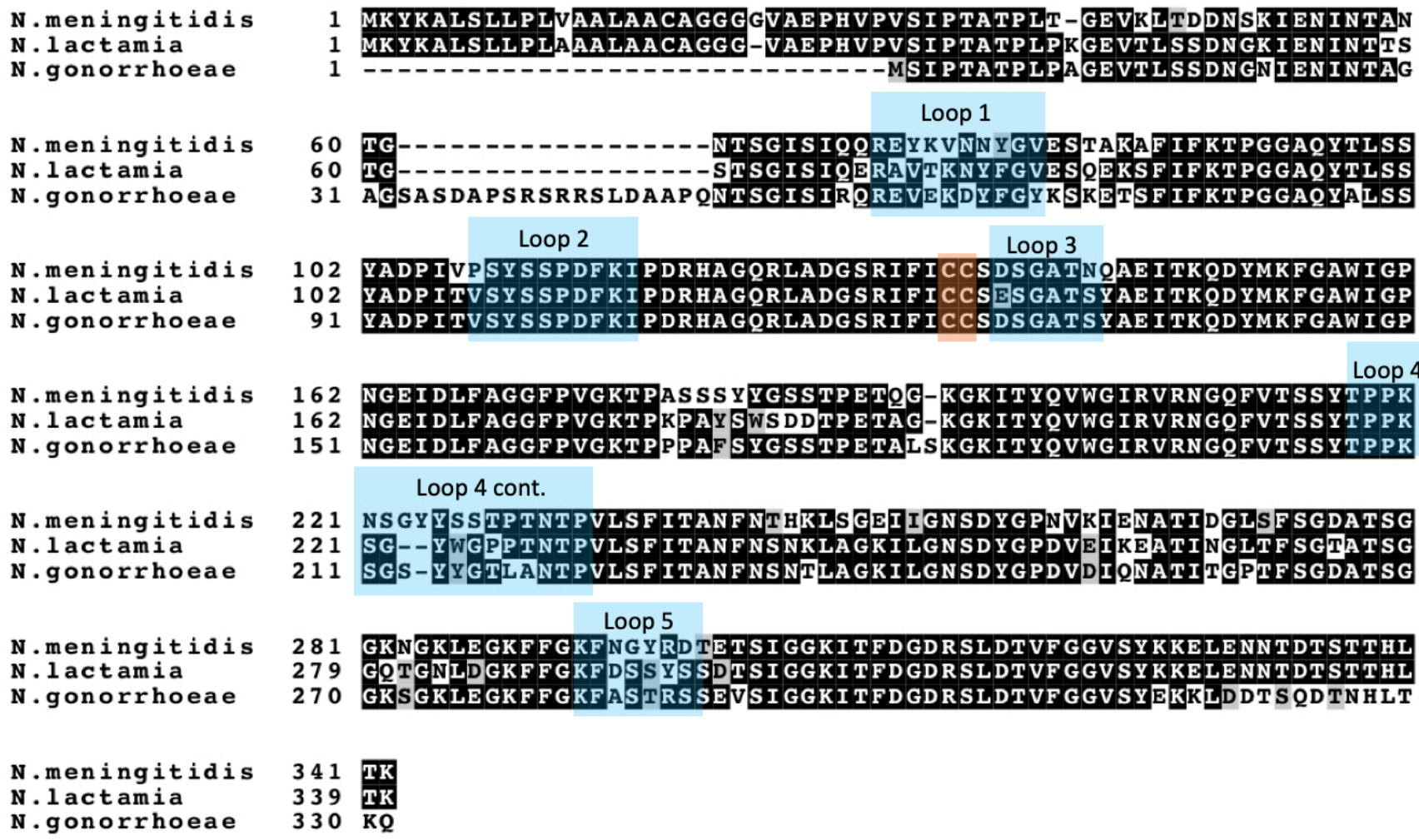

Figure 6.14. Protein sequence alignment of HpuA homologues. N. meningitidis NMBG2136, N. gonorrhoeae FA 1090, and N. lactamica 020-06 HpuA homologues were aligned using ClustalW (Thompson et al., 1994). Images created using BOXSHADE 3.21. Black shading indicates conserved residues, grey shading indicates conservative variation. Orange shading indicates conserved Cys-Cys motif. Structural loops indicated by blue boxes.

This work demonstrates there are many conserved residues among HpuA from different $N$. meningitidis isolates, and also among homologues from pathogenic and commensal Neissieria species. There was also variation in the HpuA amino acid sequences, particularly in exposed structural loops, likely due to antibody selection. Complete conservation of the Cys-Cys loop motif was found in all HpuA homologues from Neisseria species tested, in spite of the fact that these residues do not have a known structural role (Wong et al., 2015). The discovery that this Cys-Cys motif is so highly conserved, and does not impact haemoglobin binding, suggests a secondary function, which could involve adherence to host cells. Future experiments may clarify which parts of HpuA play a role in cell adhesion, and whether all HpuA homologues have this function. 


\subsection{Discussion}

The goal of this chapter was to determine how HpuA plays a role in the inhibition of respiratory epithelial cell migration by the disease-associated isolate NZ97/052. Iron acquisition from haemoglobin and haptoglobin-haemoglobin is the reported function of the HpuAB bipartite outer membrane receptor, and evidence suggests it may be key in the mucosal epithelium (Lewis et al., 1999; Tauseef et al., 2011). Examination of the literature suggested that haptoglobin may be important in wound repair (Garibay-Cerdenares et al., 2015). As HpuAB is involved with binding haptoglobin, the main hypothesis was that haptoglobin binding by HpuAB affected wound repair of host cells.

It was first hypothesised that the disease-associated isolate, expressing HpuA, could bind haptoglobin and haptoglobin-haemoglobin complexes, preventing respiratory epithelial cells from utilising haptoglobin in cell migration. This theory was based on evidence of a link between haptoglobin and cell migration (de Kleijn et al., 2002; Garibay-Cerdenares et al., 2015; Maffei et al., 2009). This chapter describes experiments undertaken to determine if supplementation with human haptoglobin could affect migrating $16 \mathrm{HBE}$ cells, with or without co-cultured meningococcal isolates. Although there was an improvement in cell migration of 16HBE cells infected with NZ97/052, this modest difference was only seen with a very high haptoglobin concentration of $1000 \mathrm{ng} / \mathrm{mL}$. Therefore, it is unlikely the difference in wound repair can be explained by HpuAB binding free haptoglobin and starving $16 \mathrm{HBE}$ cells. Haptoglobin supplementation did not have any effect on uninfected epithelial cells, which implies haptoglobin is not limiting, nor does it increase rates of cell migration at the concentrations tested. This result is in contrast with data presented by Garibay-Cerdenares et al, which reported haptoglobin supplementation increased cell migration of ovarian cells (2015). HpuAB also binds haemoglobin, therefore, there was an attempt to investigate whether supplementing cell migration assays with bovine haemoglobin could overcome the inhibition of cell migration by meningococcal isolates expressing HpuAB. However, these experiments were abandoned due to technical limitations. 
An ELISA was used to measure, over a time course, haptoglobin secreted into the cell culture medium by $16 \mathrm{HBE}$ cells in response to meningococcal isolates. The aim was to identify whether the different isolates differentially affected haptoglobin consumption or secretion by $16 \mathrm{HBE}$ cells. No significant differences were seen. Bovine haptoglobin levels were also measured, as there is some evidence that meningococci are able to utilise bovine haptoglobin (Omer et al., 2011). Results from these experiments suggested that it is very unlikely that the differences in inhibition of cell migration between isolates NZ97/052 and NZ97/052 $\Delta h p u A$ were due to haptoglobin-HpuAB binding or 'starving' 16HBE cells. This experiment did not test how much haem or iron each haptoglobin molecule was carrying, but results, along with migration assays of haptoglobin-supplemented cells, suggest that haptoglobin does not play a key role in the inhibition of wound repair by $N$. meningitidis.

Experiments were completed to investigate whether haptoglobin was co-localised with the site of bacterial attachment. Fluorescence microcopy was utilized; fixed slides were stained with a primary antibody specific for human haptoglobin. Cytoplasmic haptoglobin has been reported in many different cell types, but the significance of this is currently unknown (Theilgaard-Mönch et al., 2006). The results indicated a slight increase of cytoplasmic haptoglobin when NZ97/052 was present. However, there were very few bacteria adhered to the $16 \mathrm{HBE}$ cells for both the NZCM112 and NZ97/052 $\triangle$ hpuA isolates. A Western blot or ELISA of cellular lysates may be able to measure total cytoplasmic haptoglobin levels more precisely. Due to the differences in numbers of adherent bacteria, no conclusions could be drawn about whether there was colocalisation between N. meningitidis isolates and haptoglobin. Further experiments were designed to investigate the dramatic difference in the numbers of adherent bacteria between the isolates. It was hypothesised this result could be due to killing or growth inhibition by haptoglobin, inherent growth differences, or varying ability to adhere to host cells.

Growth curve results revealed a slightly lower final concentration of bacteria for NZ97/052 $\Delta h p u A$, compared to NZ97/052. However, viable bacterial CFU did not significantly differ between NZ97/052 $\triangle h p u A$ and wild-type NZ97/052. Therefore, this difference in adhesion is not 
likely explained by increased numbers of bacteria. These data also suggest further variations in NZCM112 contribute to its lower growth rate, as HpuA only partially accounts for the difference in growth.

It was considered that haptoglobin may have an antimicrobial effect on certain isolates. Bacteria which express HpuAB may be able to bind haptoglobin and have inherent resistance to any antimicrobial effects. It was hypothesised that haptoglobin might affect NZCM112 and NZ97/052 $\triangle h p u A$ isolates more than the wild-type NZ97/052. This hypothesis was based on evidence that haptoglobin can function as an antimicrobial agent against Gram-negative bacteria, including Proteus mirabilis and Pseudomonas aeruginosa (Board-Davies et al., 2015; Dobryszycka, 1997; Eaton et al., 1982). The antimicrobial effects of increasing concentrations of human haptoglobin on $N$. meningitidis were investigated, and it was found that haptoglobin did not have an antimicrobial effect on any of the isolates tested. In contrast to the study by BoardDavies et al (2015), where haptoglobin supplementation reduced bacterial numbers of $P$. mirabilis and $P$. aeruginosa by $50 \%$, relative to no haptoglobin, results collected in this chapter indicated that haptoglobin had no detectable antimicrobial effect on N. meningitidis.

The possibility that the difference in bacterial numbers seen in fluorescent microscopy was due to varying levels of adhesion to host cells was investigated next. Association assays described in Chapter Three showed NZ97/052 associated with 16HBE cells at higher rates than NZCM112. To examine whether HpuA is involved in adhesion, the NZ97/052 $\triangle h p u A$ isolate was compared to the wild-type NZ97/052 and NZCM112. The association assay measured levels of adherent and intracellular bacteria. The levels of intracellular bacteria were very low, so this assay can be used as a proxy to estimate cellular adherence. Adherence of NZ97/052 to respiratory epithelial cells was significantly reduced when the hpuA gene was deleted. Adherence was further examined by fluorescence microscopy, using DAPI to visualise nuclei and bacteria. Fluorescence microscopy confirmed the results seen in the association assays; the disease-associated isolate NZ97/052 had much higher levels of adhesion to 16HBE cells than NZCM112 or NZ97/052 $\Delta h p u A$. These results suggest a novel function for HpuA as a bacterial adhesin. The microscopy 
also suggested that the HpuA-expressing isolate, NZ97/052, adhered to host cells in clumps, forming many microcolonies. This result could suggest an additional role for HpuA in biofilm formation or auto-aggregation, aspects of HpuA biology as yet completely unexplored.

Adherence to respiratory epithelial cells is essential for meningococcal colonisation, although the importance of adhesion in vivo, and its potential role in invasive disease is not fully understood. There are several characterised adhesins in N. meningitidis (de Vries et al., 1998; Hung \& Christodoulides, 2013; Nagele et al., 2011), however adhesin activity of phase variable HpuA has previously never been proposed. Outer membrane proteins can be highly immunogenic. To maintain balance between evasion of the immune system and adherence to host cells, the expression of many bacterial adhesins is phase variable (Henderson et al., 1999). Transient loss of adhesins, by phase variation, could aid in transmission of the bacteria. Bacteria which detach from a colonised surface could be more likely to be transmitted to another host, through coughing or sneezing. Perhaps the "off" state of hpuA may aid in transmission, and the "on" state may aid in colonisation.

HpuA was heterologously expressed in E. coli to confirm whether this protein is sufficient, by itself, to promote attachment to epithelial host cells. A synthetic codon optimised hpuA gene cloned into a pET28a expression vector was used to transform BL21 E. coli. Successfully transformed bacteria were able to successfully grow on medium containing kanamycin. Protein expression in pET28a was induced by IPTG, and glucose was used to prevent basal gene expression in the pET system. Hi-Control BL21 cells were used, which have stringent control over a T7 lac promoter, minimising unwanted expression. Expression of HpuA by E. coli was successfully confirmed by analysis of total cell protein fraction on an SDS-PAGE. Growth rate comparison was conducted between E. coli, induced to express $\mathrm{HpuA}$, and E. coli which had expression repressed with glucose. There was a significant difference in growth after 90 minutes. E. coli used in association assays were induced for only one hour. At this point, growth rates had not significantly diverged, but this time was sufficient for expression of HpuA. There were significantly higher levels of adherence to $16 \mathrm{HBE}$ cells by $E$. coli which expressed $\mathrm{HpuA}$, 
relative to bacteria which did not express HpuA. This exciting result supports a role for HpuA in the adherence of $N$. meningitidis to host cells. The previous hypothesis, that HpuA affects wound repair by binding haptoglobin, was not supported by data collected in this chapter; rather, results suggested HpuA has a direct impact by binding to host cells.

A structural analysis of HpuA in the Neisseriae family was completed by Wong et al (2015). A highly conserved Cys-Cys motif was noted in HpuA. Mutations in this Cys-Cys loop did not affect affinity of HpuA to haemoglobin, therefore, it is not essential in haemoglobin binding. As this motif was completely conserved, they concluded that this motif is functional, and they hypothesised an unknown dual function for HpuA. Alignment of HpuA homologues from $N$. meningitidis, $N$. gonorrhoeae, and N. lactamica demonstrate that this Cys-Cys motif is completely conserved. In the future, to investigate whether this Cys-Cys motif is essential for adhesion, mutations could be made in the HpuA-pET28a vector. The cystines could be changed to serines or alanines, and association assays could test whether the $E$. coli isolate, induced to express altered HpuA, is still adherent. A Cys-Cys motif is found in the same location in TbpB and $\mathrm{LbpB}$, and further experiments must be conducted to detect whether these proteins also have any adhesin activity (Wong et al., 2015).

Typically, external loops of a protein are conserved due to functional requirement, however, in HpuA considerable variation was seen in these loops. This is presumably due to selective pressure by the host immune system. As these loops vary significantly, it poses the question of whether all HpuA alleles in N. meningitidis act as adhesins or whether only a subset have this activity. It is currently unknown how widespread this secondary adhesin function of HpuA is. Future work should examine whether carriage isolates, which do not inhibit wound repair, also lack HpuA. Genome and transcriptome analysis could identify whether these isolates have and express HpuA, which would help answer whether only some, or all alleles of hpuA have an adhesin function. Further research is required to investigate whether the external protein loops are important for HpuA-mediated adherence. If the adhesin activity lies within these loops, this 
can be tested by site-directed deletion of individual loops in the HpuA-pET28a vector, and assessing adherence to epithelial cells.

Additional future experiments would determine whether hpuA alleles from other Neisseriaceae mediate adherence to host cells. This is especially relevant for $N$. gonorrhoeae, which also expresses HpuA, but has a different niche and infection strategy than N. meningitidis. HpuA is likely the main haemoglobin receptor of $N$. gonorrhoeae, as the $h m b R$ gene contains a premature stop codon in all isolates tested (Chen et al., 1996; Stojiljkovic et al., 1996). HpuA homologues could be deleted from these bacteria, and association assays could be performed to compare cell adhesion of gene deletion mutants to the wild-type parent strain. Alleles of hpuA, which function as an adhesin, could be compared to those which do not, to help identify which domains may be important for adherence.

Moonlighting proteins are multifunctional proteins which perform multiple, often unrelated, functions (Henderson \& Martin, 2011; Huberts \& van der Klei, 2010). There are many examples of moonlighting proteins involved in iron acquisition. IsdA in Staphylococcus aureus is a haptoglobin-haemoglobin binding protein which also functions as an adhesin to fibrinogen and fibronectin (Clarke et al., 2004; Dryla et al., 2003; Foster et al., 2014). A Streptococcus pyogenes protein, HupY, is a haem binding protein which affects intracellular iron concentrations. This protein has also been shown to act as an adhesin, and may bind E-cadherin receptor or human scavenger receptor gp340 (Cook et al., 2019). Shr is another S. pyogenes surface protein, which acquires haem from haemoglobin, and also binds fibronectin and laminin (Dahesh et al., 2012; Fisher et al., 2008). Lmb is a Streptococcus agalactiae protein which acquires zinc, and also mediates adherence to human laminin (Ragunathan et al., 2013; Spellerberg et al., 1999). Finally, Haemophilus influenzae protein $\mathrm{E}(\mathrm{PE})$ is an outer membrane protein which binds to extracellular matrix proteins vitronectin and laminin, and has also been shown to bind haem (Rodríguez-Arce et al., 2019; Singh et al., 2013). Research completed in this thesis proposes a moonlighting function for HpuA as an adhesin, separate from its known activity as a haptoglobin-haemoglobin receptor. It is currently unknown what HpuA adheres to, however, 
several moonlighting haem or zinc uptake proteins bind to the extracellular matrix. Extracellular matrix proteins are important in wound repair by functioning as a scaffold for migrating cells (Badylak, 2002). N. meningitidis has been shown to bind to extracellular matrix proteins, but no adhesins have yet been reported (Eberhard et al., 1998). Future research must be conducted to determine whether HpuA binds to an extracellular matrix component, which could explain how HpuA not only mediates adherence but also interferes with host cell migration. The proposed adherence and haem binding dual functions of HpuA could enable the bacteria to adhere tightly to epithelial cells and to access intracellular haptoglobin or other iron stores. Fluorescent microscopy images indeed suggest that NZ97/052 may be adhering and "feeding" from respiratory epithelial cells. The transcriptome data further suggests that $\mathrm{HpuA}$ is actively acquiring iron for the bacteria in the cell culture model.

Results from experiments described in this chapter suggest HpuA is having a direct impact on host cells and is acting as an adhesin, rather than starving cells of haptoglobin, as originally proposed. This novel function for HpuA leads to many further questions. Future work must be completed to determine the host cell receptor, how binding to respiratory epithelial cells impacts migration, and which domains of HpuA are important for cellular adhesion.

\subsection{Summary}

In Chapter Five, deletion of hpuA was shown to abrogate the ability of disease-associated isolates to inhibit host cell wound repair. Results described in this chapter further characterised the NZ97/052 $\triangle h p u A$ mutant, and investigated how the hpuA gene impacts cell migration. Measurements of secreted haptoglobin, antimicrobial assays, and supplemented cell migration assays were completed. These results together suggest that haptoglobin does not play a key role in wound repair inhibition by $N$. meningitidis. Rather, association assays comparing wildtype NZ97/052 and NZ97/052 $\triangle h p u A$, revealed that the deletion of $h p u A$ drastically reduced bacterial attachment to host cells. These results were confirmed with fluorescence microscopy. Furthermore, heterologous expression of HpuA in E. coli increased bacterial association to 
16HBE cells. These exciting results suggests a novel role for HpuA as a meningococcal adhesion and a bacterial factor that inhibits host cell migration. 


\section{Conclusions}

Invasive meningococcal disease (IMD) is a global concern and a frightening illness due to the rapid onset of symptoms and high mortality rate, even with prompt antibiotic treatment. Recent outbreaks in New Zealand have demonstrated the continuing need for better understanding of the disease and new interventions. The overall goal of this thesis was to shed light on what distinguishes highly virulent isolates of Neisseria meningitidis from benign isolates that are associated with asymptomatic carriage. Published work has previously demonstrated that disease-associated meningococci are able to inhibit cellular migration of respiratory epithelial cells, while carriage isolates are much more variable, some not contributing to the inhibition of migration at all (Ren \& MacKichan, 2014). This difference in cell migration inhibition was particularly interesting, as evidence suggests passage through damaged epithelial layers is a mechanism of entry into the bloodstream by meningococci (Stephens, 1999, 2009). If meningococci are able to prevent, or slow, host cell wound repair, they consequently prolong their opportunity to invade deeper tissues and access the bloodstream, which could lead to IMD. The specific goal of this research project was to identify and characterise the unknown meningococcal factor that inhibits host cell migration, and to understand how it contributes to bacterial virulence.

Limiting the impact of a disease through preventive measures requires a thorough understanding of the bacterial traits that aid survival and proliferation in the human host. Understanding essential bacterial factors that are important in colonisation and survival have formed the basis of extremely effective vaccines. There are many environmental and host factors which are known to impact the development of IMD, however, bacterial factors can also clearly contribute. Understanding interactions at the mucosal surface and identifying factors which may lead to virulence is paramount for developing new interventions to prevent or mitigate disease outbreaks. 


\subsection{Research summary}

Isolates used in this thesis were collected from a single household during the meningococcal epidemic in New Zealand (Simmons et al., 2001). These closely related isolates were indistinguishable by standard laboratory typing methods. The isolate collected from the disease patient, NZ97/052, was highly effective at inhibiting epithelial cell migration. A related isolate from an asymptomatic household contact, NZCM112, did not inhibit cell migration to the same degree. This project has demonstrated the utility of studying these closely related isolates to detect meningococcal factors that may be linked to virulence, and identify genetic factors which could be associated with disease development. Experiments from this project successfully provided insights into differences between disease-associated and carriageassociated isolates.

One of the original hypotheses of this project postulated that pathogenic isolates could be inhibiting host cell wound repair by consuming nutrients in the medium that are essential for epithelial cell migration, one of the first steps in wound repair. 16HBE cells were infected with N. meningitidis isolates, and supplemented with nutrients important for bacterial growth and host cell migration. Results from these various assays suggested that this theory is likely incorrect. Supplementation with iron, zinc and non-essential amino acids had no significant effect on the inhibition of cell migration by NZ97/052. However, supplementing the carriageassociated isolate NZCM112 with iron, either as iron sulfate $\left(\mathrm{Fe}^{2+}\right)$ or iron chloride $\left(\mathrm{Fe}^{3+}\right)$, surprisingly resulted in the bacteria gaining the ability to inhibit wound repair.

Genome and transcriptome comparisons were carried out to compare the disease-associated isolate, NZ97/052, and the carriage-associated isolate, NZCM112. Genome sequencing of NZ97/052 and NZCM112 revealed two nearly identical genome sequences. Only one sequence variation was predicted to affect expression of a protein, a haptoglobin-haemoglobin outer membrane receptor, HpuA. The NZCM112 sequence had a frameshift in the hpuA gene, resulting in a premature stop codon. Transcriptome analysis confirmed the mutation in hpuA resulted in reduced expression of not only $h p u A$, but also the second gene in the operon, $h p u B$. 
Transcriptome data also identified a striking increase in the expression of multiple transcripts in NZCM112, which encoded proteins involved in the acquisition of iron from transferrin, lactoferrin, ferrous iron, and siderophores from other bacteria. This suggested that HpuA and $\mathrm{HpuB}$ are important for iron acquisition in an in vitro co-culture model, in contrast to their proposed role in the bloodstream.

A handful of candidate genes, including $h p u A$, were deleted by allelic replacement from the naturally competent NZ97/052 isolate. Successful gene deletion was confirmed by PCR product size and Sanger sequencing. Gene deletion mutants were tested for their ability to inhibit migration of respiratory epithelial cells and were compared to the wild-type parent isolate. Deletion of hpuA gene completely nullified the ability of NZ97/052 isolates to inhibit cell migration, identifying HpuA as a meningococcal factor which affects wound repair inhibition. As was seen with other carriage isolates, the addition of iron restored the ability of the NZ97/052 $\triangle h p u A$ mutant to inhibit cell migration, restoring the initial phenotype of NZ97/052 (without hpuA mutation). This implicates a second unidentified factor, in addition to $h p u A$, that can inhibit host cell migration. This second factor appears to be masked when HpuA is present and expressed, but also seems to be induced under iron-supplemented conditions.

The function of HpuA in wound repair inhibition was assessed with a focus on haptoglobin, due to evidence in the literature that haptoglobin may be linked to cell migration (de Kleijn et al., 2002; Garibay-Cerdenares et al., 2015). Work presented here, including supplemented cell migration assays, fluorescence microscopy of haptoglobin localisation, and assays measuring free haptoglobin suggested the activity of HpuA in cell migration inhibition is separate from its role in binding haptoglobin. Further characterisation of the NZ97/052 $\Delta h p u A$ mutant revealed no significant growth defects compared to the wild-type parent isolate. Association assays, measuring bacterial adhesion to host cells, demonstrated that the NZ97/052 $\triangle$ hpuA mutant had significantly lower levels of association with 16HBE epithelial cells; both adherence and invasion. This result was confirmed using fluorescence microscopy, visualising bacterial and host cell nuclei. To further investigate these results and determine if HpuA does indeed 
facilitate attachment of bacteria to respiratory epithelial cells, HpuA was heterologously expressed in E. coli. Association assays confirmed increased bacterial adherence of $E$. coli expressing HpuA. These exciting results present a novel moonlighting function for the protein HpuA as a bacterial adhesin.

\subsection{Implications of this research}

Bacterial adhesins are important virulence factors (Klemm \& Schembri, 2000). Without attachment to, and colonisation of mucosal surfaces, invasive disease would not progress. This is particularly true in the upper airways, where the ciliary escalator is constantly moving the mucous layer upward and away from the lungs. Tight and specific adherence to host cells is an important initial step for IMD, but virulence is polygenic in N. meningitidis. Invasive meningococci must also evade phagocytosis and destruction by host complement, and acquire sufficient iron in order to replicate rapidly in the bloodstream. It is unclear if NZCM112 expressing HpuA would be capable of causing invasive disease, as many other genes important for virulence are expressed by NZ97/052. Phase and antigenic variation of genes, such as HpuA or the pilus gene, have been documented to occur during infection (Omer et al., 2011; Swanson et al., 1992).

Identifying HpuA as an adhesin is a paradigm shift to the known functionality of this protein. Adhesins are often excellent targets for disease interventions and anti-adhesion therapy offers a potential alternative to antibiotic treatment. With this novel drug development approach, inhibitors are designed to bind to bacterial adhesins, which prevents them from interacting with components on the host cells (Cozens \& Read, 2012; Krachler \& Orth, 2013; Ofek et al., 2003). Blocking the adhesion of meningococci could result in the reduction of carriage and disease rates. The major drawback of this therapy is Neisseria encode more than one adhesin. Therefore, in order to be effective, multiple agents would likely be required to inhibit each type of adhesin. 
Results presented in this thesis demonstrate that HpuA expression enables $N$. meningitidis to tightly adhere to epithelial cells. Discovery of the adhesin function may also provide opportunities for much needed novel treatments for the related pathogen, Neisseria gonorrhoeae (gonococci), as HpuAB is also expressed by N. gonorrhoeae (Chen et al., 1998; Cornelissen \& Hollander, 2011). The HpuAB receptor is likely the main haemoglobin receptor of gonococci, as the $h m b R$ gene contains a premature stop codon in all isolates tested (Chen et al., 1996; Stojiljkovic et al., 1996). Interestingly, HpuAB is disproportionally expressed in women experiencing menses, which confirms the importance of this protein for growth in a haemoglobin rich environment (Anderson et al., 2001). In a healthy female genital tract, the mechanisms for how gonococci establish an association with the host cells is not fully understand (Hung \& Christodoulides, 2013). This thesis provides evidence which suggests that HpuA could also act as an adhesin in N. gonorrhoeae, promoting attachment to epithelial cells, but this remains to be tested experimentally.

$N$. gonorrhoeae is the causative agent of gonorrhoea, and is one of the most common causes of sexually transmitted infections. Gonorrhoea incidence is increasing worldwide (WHO, 2012a). Multi-drug resistant strains are developing, and there is fear this bacteria may evolve resistance to all major classes of antimicrobial therapy (Tapsall et al., 2009; Unemo \& Shafer, 2014; Wang et al., 2003). Highly antibiotic resistant strains are already circulating in vulnerable populations. Infections caused by these strains often require hospitalisation and intravenous antibiotic delivery, amplifying the economic and social cost of this disease. Increasing cases of untreatable $N$. gonorrhoeae has resulted in an urgent need to focus on the developments of new targets for antimicrobial treatments. Prevention of disease is always better than cure, but vaccine development for N. gonorrhoeae has been problematic (Edwards et al., 2018; Jerse et al., 2014). There has been some evidence which suggests meningococcal $B$ vaccines MeNZB and Bexsero may provide cross-protection against gonorrhoea, although efficacy is low (PetousisHarris et al., 2017; Semchenko et al., 2018). The surface expression of HpuAB receptors makes it a promising target for vaccine development. The phase variable nature of the outer membrane protein HpuA could complicate efficacy as a vaccine target, although levels of 
expression of the HpuAB receptor in both disease and carriage isolates remain high (Tauseef et al., 2011). As a result, this protein could be an effective target in a multi component vaccine. Anti-adhesion therapy could also be developed as a new means to fight infections, by interfering with bacterial adherence.

\subsection{Limitations of research using N. meningitidis}

N. meningitidis is an airborne pathogen, therefore, all research must be undertaken with prudent controls in place. Manipulations of clinical isolates, that may produce aerosols, can lead to laboratory-acquired infections if proper protocols are not carefully followed. Prevention of infection should focus on the use of bio-safety cabinets during manipulations (Borrow et al., 2014). In order to work safely with N. meningitidis, and prevent the formation of aerosols, there are limitations to the types of experiments that can be completed, depending on laboratory set up. In some instances in this research project some ideal experimental approaches were precluded due to safety considerations, such as continuously monitoring the bacterial growth by optical density over a time course. However, a plate reader was not available to be used in the bio-safety cabinet at ESR.

\subsection{Future directions of this research}

It is clear the process of wound repair inhibition by N. meningitidis is very complex, and this project has raised many questions in addition to answers. The link between cell attachment and prevention of host cell wound repair remains elusive. The experiments in this thesis clearly showed HpuA has an effect on both adherence and inhibition of wound repair, but how bacterial attachment impacts the migration of host cells remains as yet unknown. Future directions of this work could investigate the role of HpuA as an adhesin, and determine the cellular target of binding.

Many of the moonlighting haem or zinc acquisition proteins in other species of bacteria frequently bind to extracellular matrix (ECM) proteins. These basement membrane proteins are 
likely exposed during epithelial cell disruption, and many pathogenic bacteria adhere to ECM proteins. Apart from helping the pathogen to adhere to host tissues, ECM components, such as vitronectin, also inhibit deposition of the complement membrane attack complex, protecting vitronectin-coated bacteria from complement killing. It is possible that Neisseria HpuA, and other proteins that play a role in iron acquisition, are also moonlighting as adhesins that bind components of the extracellular matrix. N. meningitidis has previously been shown to bind to ECM proteins, especially fibronectin and collagen (Eberhard et al., 1998). Extracellular matrix proteins are important in cell migration, and if $\mathrm{HpuA}$ binds to a component of the extracellular matrix, this could explain how HpuA both mediates adherence and also interferes with host cell migration. To research this further, an experiment could be conducted to test whether isolates expressing HpuA are able to adhere to glass slides which have been coated with extracellular matrix proteins, as described in Eberhard et al (1998).

A highly conserved Cys-Cys domain has previously been discovered in HpuA, which is not involved in haem binding. As this motif is so highly conserved, it indicates a secondary function for the gene (Wong et al., 2015). This domain may be responsible for the adhesin activity of HpuA. Mutations could be designed in the Cys-Cys domain, and epithelial cell binding could be measured to confirm whether this highly conserved domain is involved in bacterial attachment to host cells. Alternatively, the adhesin function of HpuA could lie in one of the external protein loops. If the adhesin activity lies within these loops, this can be tested by site-directed deletion of individual loops in the HpuA-pET28a vector, and assessing adherence to epithelial cells. The external protein loops of HpuA vary considerably between $N$. meningitidis isolates, presumably due to selective pressure by the host immune system, and it remains to be tested whether all, or only a subset, of hpuA alleles act as an adhesin. Genome and transcriptome analysis of carriage isolates, which do not inhibit wound repair under normal conditions, could establish whether these isolates also lack HpuA. If any of these isolates express $\mathrm{HpuA}$, the sequence could be compared to the HpuA sequence in NZ97/052 to help identify the sequence or external protein loop which may be important in HpuA-mediated adherence. It will also be 
important to determine whether the $N$. gonorrhoeae hpuA variant mediates adherence to epithelial cells, similar to the $N$. meningitidis allele.

To explore what other meningococcal factors contribute to wound repair inhibition, the effects of iron supplementation on carriage isolates should be further investigated. Addition of iron to isolates, which do not normally inhibit wound repair, caused significant increases in wound repair inhibition, while it did not alter the migration of uninfected respiratory epithelial cells. This phenomena also occurred in the NZ97/052 isolate with the $h p u A$ gene deleted. These results indicate there is more than one factor which can impact wound repair inhibition. Transcriptome analysis of meningococcal isolates with and without iron sulfate supplementation could be used to reveal some candidates which may be responsible for the effect on respiratory epithelial cell migration. However, iron is known to be extremely important for bacterial virulence, and as such many genes are iron regulated (Gozzelino \& Arosio, 2015; Skaar, 2010; Wooldridge \& Williams, 1993).

A study by Grifantini et al (2003) showed that a N. meningitidis group B isolate altered the expression, at least 2-fold, of 235 genes in the presence of iron. This equates to approximately $11 \%$ of the meningococcal genome. Therefore, a transcriptome comparison with and without iron supplementation could reveal a very large list of genes. Multiple carriage isolates, which cause increased wound repair inhibition in the presence of iron, could be compared to identify candidate genes which differ in expression across all isolates. A literature review could also narrow down the list of meningococcal genes which may interact with host cells, or have an impact on cell migration. Preliminary BLAST queries identified a possible laminin binding site on HpuB. This was found by searching the NMBG2136 genome (Budroni et al., 2011) with the reported laminin binding motif from $H$. influenzae protein $E$ (Singh et al., 2013). A potential fibronectin binding site was also seen on the haemoglobin receptor $\mathrm{HmbR}$, following a BLAST search of NMBG2136 with the FAP-A fibronectin-binding protein from Mycobacterium avium. Furthermore, possible collagen, vitronectin, and laminin binding motifs were identified in several other putative transporter proteins in NMBG2136. The significance of these putative 
motifs remains untested and would be an exciting avenue of future research. Multiple haembinding and uptake proteins in $H$. influenzae have been reported as having moonlighting functions, including adherence, resistance to antimicrobial peptides, and import of glutathione, implying the secondary functions of surface Neisseria proteins may also turn out to be widespread (Rodriguez-Arce et al, 2019).

HpuA is also a key haemoglobin utilisation protein in N. gonorrhoeae, which could allow for future research on this protein to be conducted using this bacteria. N. gonorrhoeae is a human pathogen; although manipulations must still be completed using safe laboratory procedures (Spence et al., 2008), laboratory-acquired infections via aerosol transmission have not been documented. It is considered much safer to perform manipulations in this bacteria than $N$. meningitidis, as the consequences of infection are much less severe. Epithelial cell adherence assays of gonococci can be performed with cervical epithelial cells to determine whether the adhesin effect of HpuA occurs in a different mucosal surface. Further investigation of HpuA moonlighting functions could be explored by heterologous expression of the gonococcal HpuA in E. coli. Experiments can be performed using these bacteria, which could not be completed safely with the available resources using $N$. meningitidis.

\subsection{Conclusions}

The aim of this thesis was to identify the bacterial factor responsible for respiratory epithelial cell wound repair by disease-associated N. meningitidis isolate NZ97/052. Genome and transcriptome comparisons were completed, and a panel of candidate genes were deleted from the disease-associate isolate NZ97/052. Deletion of the hpuA gene from NZ97/052 completely nullified the ability to inhibit host cell migration, thereby enabling identification of the meningococcal factor responsible for cell migration inhibition. The results of this research suggest there is likely at least one additional meningococcal factor, expressed under certain conditions, which can affect cell migration. This second factor remains to be identified. Characterisation of NZ97/052 $\triangle$ hpuA revealed novel roles for $\mathrm{HpuA}$ as a bacterial adhesin that 
inhibits host cell migration. Future research could determine how bacterial attachment by HpuA manipulates host cell processes. 


\section{References}

Agier, L., Deroubaix, A., Martiny, N., Yaka, P., Djibo, A., \& Broutin, H. (2013). Seasonality of meningitis in Africa and climate forcing: aerosols stand out. J R Soc Interface, 10(79).

Agren, M. S. (1990). Studies on zinc in wound healing. Acta Derm Venereol, 154, 1-36.

Ala'Aldeen, D. A., Neal, K. R., Ait-Tahar, K., Nguyen-Van-Tam, J. S., English, A., Falla, T. J., Hawkey, P. M., \& Slack, R. C. (2000). Dynamics of meningococcal long-term carriage among university students and their implications for mass vaccination. J Clin Microbiol, 38(6), 2311-2316.

Altmann, G., Egoz, N., \& Bogokovsky, B. (1973). Observations on asymptomatic infections with Neisseria meningitidis. Am J Epidemiol, 98(6), 446-452.

Altschul, S. F., Gish, W., Miller, W., Myers, E. W., \& Lipman, D. J. (1990). Basic local alignment search tool. J Mol Biol, 215(3), 403-410.

Ambur, O. H., Frye, S. A., Nilsen, M., Hovland, E., \& Tønjum, T. (2012). Restriction and Sequence Alterations Affect DNA Uptake Sequence-Dependent Transformation in Neisseria meningitidis. Plos One, 7(7), e39742.

Andersen, J., Berthelsen, L., Bech Jensen, B., \& Lind, I. (1998). Dynamics of the meningococcal carrier state and characteristics of the carrier strains: a longitudinal study within three cohorts of military recruits. Epidemiol Infect, 121(1), 85-94.

Anderson, G. J., \& Frazer, D. M. (2017). Current understanding of iron homeostasis. Am J Clin Nutr, 106(Suppl 6), 1559s-1566s.

Anderson, J. E., Leone, P. A., Miller, W. C., Chen, C., Hobbs, M. M., \& Sparling, P. F. (2001). Selection for expression of the gonococcal hemoglobin receptor during menses. $J$ Infect Dis, 184(12), 1621-1623.

Andreini, C., Bertini, I., Cavallaro, G., Holliday, G. L., \& Thornton, J. M. (2008). Metal ions in biological catalysis: from enzyme databases to general principles. J Biol Inorg Chem, 13(8), 1205-1218.

Andrews, N. C. (1999). Disorders of Iron Metabolism. N Engl J Med, 341(26), 1986-1995.

Andrews, S. C., Robinson, A. K., \& Rodríguez-Quiñones, F. (2003). Bacterial iron homeostasis. FEMS Microbiol Rev, 27(2-3), 215-237.

Archibald, F. S., \& DeVoe, I. W. (1979). Removal of iron from human transferrin by Neisseria meningitidis. FEMS Microbiol Lett, 6(3), 159-162. 
Archibald, F. S., \& DeVoe, I. W. (1980). Iron acquisition by Neisseria meningitidis in vitro. Infect Immun, 27(2), 322-334.

Badylak, S. F. (2002). The extracellular matrix as a scaffold for tissue reconstruction. Semin Cell Dev Biol, 13(5), 377-383.

Bagg, A., \& Neilands, J. B. (1987). Molecular mechanism of regulation of siderophore-mediated iron assimilation. Microbiol Rev, 51(4), 509-518.

Baraff, L. J., Lee, S. I., \& Schriger, D. L. (1993). Outcomes of bacterial meningitis in children: a meta-analysis. Pediatr Infect Dis J, 12(5), 389-394.

Barber, R. C., Aragaki, C. C., Rivera-Chavez, F. A., Purdue, G. F., Hunt, J. L., \& Horton, J. W. (2004). TLR4 and TNF- $\alpha$ polymorphisms are associated with an increased risk for severe sepsis following burn injury. J Med Genet, 41(11), 808.

Beddek, A. J., Li, M. S., Kroll, J. S., Jordan, T. W., \& Martin, D. R. (2009). Evidence for capsule switching between carried and disease-causing Neisseria meningitidis strains. Infect Immun, 77(7), 2989-2994.

Bernstein, J. A., Khodursky, A. B., Lin, P. H., Lin-Chao, S., \& Cohen, S. N. (2002). Global analysis of mRNA decay and abundance in Escherichia coli at single-gene resolution using twocolor fluorescent DNA microarrays. Proc Natl Acad Sci U S A, 99(15), 9697-9702.

Beucher, M., \& Sparling, P. F. (1995). Cloning, sequencing, and characterization of the gene encoding FrpB, a major iron-regulated, outer membrane protein of Neisseria gonorrhoeae. J Bacteriol, 177(8), 2041-2049.

Bidmos, F. A., Neal, K. R., Oldfield, N. J., Turner, D. P., Ala'Aldeen, D. A., \& Bayliss, C. D. (2011). Persistence, replacement, and rapid clonal expansion of meningococcal carriage isolates in a 2008 university student cohort. J Clin Microbiol, 49(2), 506-512.

Biville, F., Brezillon, C., Giorgini, D., \& Taha, M. K. (2014). Pyrophosphate-mediated iron acquisition from transferrin in Neisseria meningitidis does not require TonB activity. Plos One, 9(10), e107612.

Board-Davies, E., Moses, R., Sloan, A., Stephens, P., \& Davies, L. C. (2015). Oral Mucosal Lamina Propria-Progenitor Cells Exert Antibacterial Properties via the Secretion of Osteoprotegerin and Haptoglobin. Stem Cells Transl Med, 4(11), 1283-1293.

Borrow, R., Findlow, J., Gray, S., Taylor, S., \& Kaczmarski, E. (2014). Safe laboratory handling of Neisseria meningitidis. J Infect, 68(4), 305-312.

Brandtzaeg, P., Halstensen, A., Kierulf, P., Espevik, T., \& Waage, A. (1992). Molecular mechanisms in the compartmentalized inflammatory response presenting as meningococcal meningitis or septic shock. Microb Pathog, 13(6), 423-431. 
Broome, C. V. (1986). The carrier state: Neisseria meningitidis. J Antimicrob Chemother, 18, 2534.

Brown, S. P., Cornforth, D. M., \& Mideo, N. (2012). Evolution of virulence in opportunistic pathogens: generalism, plasticity, and control. Trends Microbiol, 20(7), 336-342.

Brzoska, K., Meczynska, S., \& Kruszewski, M. (2006). Iron-sulfur cluster proteins: electron transfer and beyond. Acta Biochim Pol, 53(4), 685-691.

Budroni, S., Siena, E., Hotopp, J. C. D., Seib, K. L., Serruto, D., Nofroni, C., Comanducci, M., Riley, D. R., Daugherty, S. C., Angiuoli, S. V., Covacci, A., Pizza, M., Rappuoli, R., Moxon, E. R., Tettelin, H., \& Medini, D. (2011). Neisseria meningitidis is structured in clades associated with restriction modification systems that modulate homologous recombination. PNAS, 108(11), 4494-4499.

Capecchi, B., Adu-Bobie, J., Di Marcello, F., Ciucchi, L., Masignani, V., Taddei, A., Rappuoli, R., Pizza, M., \& Arico, B. (2005). Neisseria meningitidis NadA is a new invasin which promotes bacterial adhesion to and penetration into human epithelial cells. $\mathrm{Mol}$ Microbiol, 55(3), 687-698.

Carbonnelle, E., Helaine, S., Nassif, X., \& Pelicic, V. (2006). A systematic genetic analysis in Neisseria meningitidis defines the Pil proteins required for assembly, functionality, stabilization and export of type IV pili. Mol Microbiol, 61(6), 1510-1522.

Carson, S. D., Klebba, P. E., Newton, S. M., \& Sparling, P. F. (1999). Ferric enterobactin binding and utilization by Neisseria gonorrhoeae. J Bacteriol, 181(9), 2895-2901.

Cartwright, K. A. V., Jones, D. M., Kaczmarski, E., Smith, A. J., Stuart, J. M., \& Palmer, S. R. (1991). Influenza A and meningococcal disease. Lancet, 338(8766), 554-557.

Caugant, D. A., Bovre, K., Gaustad, P., Bryn, K., Holten, E., Hoiby, E. A., \& Froholm, L. O. (1986). Multilocus genotypes determined by enzyme electrophoresis of Neisseria meningitidis isolated from patients with systemic disease and from healthy carriers. J Gen Microbiol, 132(3), 641-652.

Caugant, D. A., Froholm, L. O., Bovre, K., Holten, E., Frasch, C. E., Mocca, L. F., Zollinger, W. D., \& Selander, R. K. (1986). Intercontinental spread of a genetically distinctive complex of clones of Neisseria meningitidis causing epidemic disease. PNAS, 83(13), 4927-4931.

Caugant, D. A., Hoiby, E. A., Rosenqvist, E., Froholm, L. O., \& Selander, R. K. (1992). Transmission of Neisseria meningitidis among asymptomatic military recruits and antibody analysis. Epidemiol Infect, 109(2), 241-253.

Caugant, D. A., Tzanakaki, G., \& Kriz, P. (2007). Lessons from meningococcal carriage studies. FEMS Microbiol Rev, 31(1), 52-63. 
Cerasi, M., Ammendola, S., \& Battistoni, A. (2013). Competition for zinc binding in the hostpathogen interaction. Front Cell Infect Microbiol, 3.

Chen, C. J., Elkins, C., \& Sparling, P. F. (1998). Phase variation of hemoglobin utilization in Neisseria gonorrhoeae. Infect Immun, 66(3), 987-993.

Chen, C. J., Sparling, P. F., Lewis, L. A., Dyer, D. W., \& Elkins, C. (1996). Identification and purification of a hemoglobin-binding outer membrane protein from Neisseria gonorrhoeae. Infect Immun, 64(12), 5008-5014.

Chiu, H. Y., Sun, K. H., Chen, S. Y., Wang, H. H., Lee, M. Y., Tsou, Y. C., Jwo, S. C., Sun, G. H., \& Tang, S. J. (2012). Autocrine CCL2 promotes cell migration and invasion via PKC activation and tyrosine phosphorylation of paxillin in bladder cancer cells. Cytokine, 59(2), 423-432.

Christensen, H., May, M., Bowen, L., Hickman, M., \& Trotter, C. L. (2010). Meningococcal carriage by age: a systematic review and meta-analysis. Lancet Infect Dis, 10(12), 853861.

Christensen, P. J., Du, M., Moore, B., Morris, S., Toews, G. B., \& Paine, R. (2004). Expression and functional implications of CCR2 expression on murine alveolar epithelial cells. Am J Physiol Lung Cell Mol Physiol, 286(1), L68-L72.

Christopher, G. W. (1985). Escherichia coli bacteremia, meningitis, and hemochromatosis. Arch Intern Med, 145(10), 1908.

Clarke, S. R., Wiltshire, M. D., \& Foster, S. J. (2004). IsdA of Staphylococcus aureus is a broad spectrum, iron-regulated adhesin. Mol Microbiol, 51(5), 1509-1519.

Claus, H., Maiden, M. C. J., Maag, R., Frosch, M., \& Vogel, U. (2002). Many carried meningococci lack the genes required for capsule synthesis and transport. Microbiology, 148, 18131819.

Claus, H., Weinand, H., Frosch, M., \& Vogel, U. (2003). Identification of the hypervirulent lineages of Neisseria meningitidis, the ST-8 and ST-11 complexes, by using monoclonal antibodies specific to NmeDI. J Clin Microbiol, 41(8), 3873-3876.

Coen, P. G., Tully, J., Stuart, J. M., Ashby, D., Viner, R. M., \& Booy, R. (2006). Is it exposure to cigarette smoke or to smokers which increases the risk of meningococcal disease in teenagers? Int J Epidemiol, 35(2), 330-336.

Collins, N. (2002). Glutamine and Wound Healing. Adv Skin Wound Care, 15(5), 233-234.

Comanducci, M., Bambini, S., Brunelli, B., Adu-Bobie, J., Arico, B., Capecchi, B., Giuliani, M. M., Masignani, V., Santini, L., Savino, S., Granoff, D. M., Caugant, D. A., Pizza, M., Rappuoli, 
R., \& Mora, M. (2002). NadA, a novel vaccine candidate of Neisseria meningitidis. J Exp Med, 195(11), 1445-1454.

Comanducci, M., Bambini, S., Caugant, D. A., Mora, M., Brunelli, B., Capecchi, B., Ciucchi, L., Rappuoli, R., \& Pizza, M. (2004). NadA diversity and carriage in Neisseria meningitidis. Infect Immun, 72(7), 4217-4223.

Cook, L. C. C., Chatterjee, N., Li, Y., Andrade, J., Federle, M. J., \& Eichenbaum, Z. (2019). Transcriptomic Analysis of Streptococcus pyogenes; Colonizing the Vaginal Mucosa Identifies hupY, an MtsR-Regulated Adhesin Involved in Heme Utilization. mBio, 10(3), e00848-00819.

Coraux, C., Hajj, R., Lesimple, P., \& Puchelle, E. (2005). In vivo models of human airway epithelium repair and regeneration. European Respiratory Review, 14(97), 131.

Cornelissen, C. N., \& Hollander, A. (2011). TonB-Dependent Transporters Expressed by Neisseria gonorrhoeae. Frontiers in microbiology, 2, 117.

Cornelissen, C. N., \& Sparling, P. F. (1994). Iron piracy: acquisition of transferrin-bound iron by bacterial pathogens. Mol Microbiol, 14(5), 843-850.

Coureuil, M., Bourdoulous, S., Marullo, S., \& Nassif, X. (2014). Invasive meningococcal disease: a disease of the endothelial cells. Trends Mol Med, 20(10), 571-578.

Coureuil, M., Join-Lambert, O., Lécuyer, H., Bourdoulous, S., Marullo, S., \& Nassif, X. (2013). Pathogenesis of Meningococcemia. Cold Spring Harb Perspect Med, 3(6), a012393.

Cozens, A. L., Yezzi, M. J., Kunzelmann, K., Ohrui, T., Chin, L., Eng, K., Finkbeiner, W. E., Widdicombe, J. H., \& Gruenert, D. C. (1994). CFTR expression and chloride secretion in polarized immortal human bronchial epithelial cells. Am J Respir Cell Mol Biol, 10(1), 3847.

Cozens, D., \& Read, R. C. (2012). Anti-adhesion methods as novel therapeutics for bacterial infections. Expert Rev Anti Infect Ther, 10(12), 1457-1468.

Crichton, R. R., \& Charloteaux-Wauters, M. (1987). Iron transport and storage. Eur J Biochem, 164(3), 485-506.

D'Armiento, J., Dalal, S. S., \& Chada, K. (1997). Tissue, temporal and inducible expression pattern of haptoglobin in mice. Gene, 195(1), 19-27.

Dahesh, S., Nizet, V., \& Cole, J. N. (2012). Study of streptococcal hemoprotein receptor (Shr) in iron acquisition and virulence of M1T1 group A streptococcus. Virulence, 3(7), 566-575.

Daures, M., John, M., Balter, C. V., Simon, O., Barguil, Y., Missotte, I., Grangeon, J. P., LaumondBarny, S., Noel, M., Besson-Leaud, L., Spasic, P. E., de Suremain, A., Gourinat, A. C., \& 
Descloux, E. (2015). Relationships Between Clinico-Epidemiological Patterns of Invasive Meningococcal Infections and Complement Deficiencies in French South Pacific Islands (New Caledonia). J Clin Immunol, 35(1), 47-55.

de Kleijn, D. P., Smeets, M. B., Kemmeren, P. P., Lim, S. K., Van Middelaar, B. J., Velema, E., Schoneveld, A., Pasterkamp, G., \& Borst, C. (2002). Acute-phase protein haptoglobin is a cell migration factor involved in arterial restructuring. Faseb j, 16(9), 1123-1125.

de Vries, F. P., Cole, R., Dankert, J., Frosch, M., \& van Putten, J. P. (1998). Neisseria meningitidis producing the Opc adhesin binds epithelial cell proteoglycan receptors. Mol Microbiol, 27(6), 1203-1212.

Deeks, E. D. (2010). Meningococcal quadrivalent (serogroups A, C, W135, and Y) conjugate vaccine (Menveo): in adolescents and adults. BioDrugs, 24(5), 287-297.

Delany, I., leva, R., Alaimo, C., Rappuoli, R., \& Scarlato, V. (2003). The Iron-Responsive Regulator Fur Is Transcriptionally Autoregulated and Not Essential in Neisseria meningitidis. $J$ Bacteriol, 185(20), 6032.

Desai, L. P., Aryal, A. M., Caecareanu, B., Hassid, A., \& Waters, C. M. (2004). RhoA and Rac1 are both required for efficient wound closure of airway epithelial cells. Am J Physiol Lung Cell Mol Physiol, 287(6), L1134-L1144.

Dobryszycka, W. (1997). Biological functions of haptoglobin--new pieces to an old puzzle. Eur J Clin Chem Clin Biochem, 35(9), 647-654.

Dryla, A., Gelbmann, D., Von Gabain, A., \& Nagy, E. (2003). Identification of a novel iron regulated staphylococcal surface protein with haptoglobin-haemoglobin binding activity. Mol Microbiol, 49(1), 37-53.

Dupuit, F., Gaillard, D., Hinnrasky, J., Mongodin, E., de Bentzmann, S., Copreni, E., \& Puchelle, E. (2000). Differentiated and functional human airway epithelium regeneration in tracheal xenografts. Am J Physiol Lung Cell Mol Physiol, 278(1), L165-176.

Dyer, D. W., West, E. P., \& Sparling, P. F. (1987). Effects of serum carrier proteins on the growth of pathogenic neisseriae with heme-bound iron. Infect Immun, 55(9), 2171-2175.

Dyet, K., Devoy, A., McDowell, R., \& Martin, D. (2005). New Zealand's epidemic of meningococcal disease described using molecular analysis: implications for vaccine delivery. Vaccine, 23(17-18), 2228-2230.

Dyet, K. H., \& Martin, D. R. (2006). Clonal analysis of the serogroup B meningococci causing New Zealand's epidemic. Epidemiol Infect, 134(2), 377-383.

Eaton, J., Brandt, P., Mahoney, \& Lee, J. (1982). Haptoglobin: a natural bacteriostat. Science, 215(4533), 691-693. 
Eberhard, T., Virkola, R., Korhonen, T., Kronvall, G., \& Ullberg, M. (1998). Binding to Human Extracellular Matrix by Neisseria meningitidis. Infect Immun, 66(4), 1791.

Edwards, J. L., Jennings, M. P., \& Seib, K. L. (2018). Neisseria gonorrhoeae vaccine development: hope on the horizon? Curr Opin Infect Dis, 31(3), 246-250.

Eisenstark, A. (1977). Genetic recombination in bacteria Annu Rev Genet, 11(1), 369-396.

Ellinger, S. (2014). Micronutrients, Arginine, and Glutamine: Does Supplementation Provide an Efficient Tool for Prevention and Treatment of Different Kinds of Wounds? Adv Wound Care, 3(11), 691-707.

Elner, S. G., Strieter, R. M., Elner, V. M., Rollins, B. J., Del Monte, M. A., \& Kunkel, S. L. (1991). Monocyte chemotactic protein gene expression by cytokine-treated human retinal pigment epithelial cells. Lab Invest, 64(6), 819-825.

Erjefalt, J. S., Erjefalt, I., Sundler, F., \& Persson, C. G. (1995). In vivo restitution of airway epithelium. Cell Tissue Res, 281(2), 305-316.

ESR. (2018a). Invasive Meningococcal Disease Report, November 23rd. Institute of Environmental Science and Research Ltd.

ESR. (2018b). Invasive Meningococcal Disease Report, December 19th Institute of Environmental Science and Research Ltd.

ESR. (2019). Invasive Meningococcal Disease Report, April 3rd. Institute of Environmental Science and Research Ltd.

Fang, W. B., Jokar, I., Zou, A., Lambert, D., Dendukuri, P., \& Cheng, N. (2012). CCL2/CCR2 chemokine signaling coordinates survival and motility of breast cancer cells through Smad3 protein- and p42/44 mitogen-activated protein kinase (MAPK)-dependent mechanisms. J Biol Chem, 287(43), 36593-36608.

Feavers, I. M., Fox, A. J., Gray, S., Jones, D. M., \& Maiden, M. C. (1996). Antigenic diversity of meningococcal outer membrane protein PorA has implications for epidemiological analysis and vaccine design. Clin Diagn Lab Immunol, 3(4), 444-450.

Feiken, E., Romer, J., Eriksen, J., \& Lund, L. R. (1995). Neutrophils express tumor necrosis factoralpha during mouse skin wound healing. J Invest Dermatol, 105(1), 120-123.

Feil, E. J., Maiden, M. C., Achtman, M., \& Spratt, B. G. (1999). The relative contributions of recombination and mutation to the divergence of clones of Neisseria meningitidis. Mol Biol Evol, 16(11), 1496-1502. 
Fernandez, J. M., Serrano, M., De Arriba, J. J., Sanchez, M. V., Escribano, E., \& Ferreras, P. (2000). Bacteremic cellulitis caused by Non-01, Non-0139 Vibrio cholerae: report of a case in a patient with hemochromatosis. Diagn Microbiol Infect Dis, 37(1), 77-80.

Finlay, B. B., \& Falkow, S. (1997). Common themes in microbial pathogenicity revisited. Microbiol Mol Biol Rev, 61(2), 136-169.

Fischer, M., Hedberg, K., Cardosi, P., Plikaytis, B. D., Hoesly, F. C., Steingart, K. R., Bell, T. A., Fleming, D. W., Wenger, J. D., \& Perkins, B. A. (1997). Tobacco smoke as a risk factor for meningococcal disease. Pediatr Infect Dis J, 16(10), 979-983.

Fisher, M., Huang, Y. S., Li, X., Mclver, K. S., Toukoki, C., \& Eichenbaum, Z. (2008). Shr is a broadspectrum surface receptor that contributes to adherence and virulence in group $A$ streptococcus. Infect Immun, 76(11), 5006-5015.

Flexner, S. (1913). The results of the serum treatment in thirteen hundred cases of epidemic meningitis. J Exp Med, 17(5), 553-576.

Foster, T. J., Geoghegan, J. A., Ganesh, V. K., \& Höök, M. (2014). Adhesion, invasion and evasion: the many functions of the surface proteins of Staphylococcus aureus. Nat Rev Microbiol, 12(1), 49-62.

Franzoso, S., Mazzon, C., Sztukowska, M., Cecchini, P., Kasic, T., Capecchi, B., Tavano, R., \& Papini, E. (2008). Human monocytes/macrophages are a target of Neisseria meningitidis Adhesin A (NadA). J Leukoc Biol, 83(5), 1100-1110.

Frosch, M., \& Maiden, M. (2006). Handbook of Meningococcal Disease. Weinheim, Germany: WileyVCH.

Frye, S. A., Nilsen, M., Tønjum, T., \& Ambur, O. H. (2013). Dialects of the DNA Uptake Sequence in Neisseriaceae. PLoS Genet, 9(4), e1003458.

Gal-Mor, O., \& Finlay, B. B. (2006). Pathogenicity islands: a molecular toolbox for bacterial virulence. Cell Microbiol, 8(11), 1707-1719.

Garibay-Cerdenares, O., Hernández-Ramírez, V., Osorio-Trujillo, J., Gallardo-Rincón, D., \& Talamás-Rohana, P. (2015). Haptoglobin and CCR2 receptor expression in ovarian cancer cells that were exposed to ascitic fluid: Exploring a new role of haptoglobin in the tumoral microenvironment. Cell Adh Migr, 9(5), 394-405.

Garrison, E., \& Marth, G. (2012). Haplotype-based variant detection from short-read sequencing. eprint arXiv:1207.3907, arXiv:1207.3907.

Gaynor, E. C., Cawthraw, S., Manning, G., MacKichan, J. K., Falkow, S., \& Newell, D. G. (2004). The genome-sequenced variant of Campylobacter jejuni NCTC 11168 and the original 
clonal clinical isolate differ markedly in colonization, gene expression, and virulenceassociated phenotypes. J Bacteriol, 186(2), 503-517.

Geiser, T., Jarreau, P. H., Atabai, K., \& Matthay, M. A. (2000). Interleukin-1beta augments in vitro alveolar epithelial repair. Am J Physiol Lung Cell Mol Physiol, 279(6), L1184-1190.

Gorringe, A. R., \& Pajon, R. (2012). Bexsero: a multicomponent vaccine for prevention of meningococcal disease. Hum Vaccin Immunother, 8(2), 174-183.

Gozzelino, R., \& Arosio, P. (2015). The importance of iron in pathophysiologic conditions. Front Pharmacol, 6, 26.

Grass, G., Wong, M. D., Rosen, B. P., Smith, R. L., \& Rensing, C. (2002). ZupT is a Zn(II) uptake system in Escherichia coli. J Bacteriol, 184(3), 864-866.

Greenwood, B. (1999). Meningococcal meningitis in Africa. Manson Lecture. . Trans R Soc Trop Med Hyg, 93(4), 341-353.

Greenwood, B. M., Blakebrough, I. S., Bradley, A. K., Wali, S., \& Whittle, H. C. (1984). Meningococcal disease and season in sub-Saharan Africa. Lancet, 1(8390), 1339-1342.

Greig, G. A. (2014). The Effect of Neisseria meningitidis Genes nadA and zupT on Respiratory Epithelial Cell Wound Repair (Unpublished honours thesis). Victoria University of Wellington, Wellington, New Zealand.

Griekspoor, A., \& Groothuis, T. (2005). 4 Peaks. Ver. 1.7.

Grifantini, R., Sebastian, S., Frigimelica, E., Draghi, M., Bartolini, E., Muzzi, A., Rappuoli, R., Grandi, G., \& Genco, C. A. (2003). Identification of Iron-Activated and-Repressed FurDependent Genes by Transcriptome Analysis of Neisseria meningitidis Group B. PNAS, 100(16), 9542-9547.

Guerinot, M. L. (2000). The ZIP family of metal transporters. Biochim Biophys Acta Biomembr, 1465(1-2), 190-198.

Guilhen, C., Taha, M. K., \& Veyrier, F. J. (2013). Role of Transition Metal Exporters In Virulence: The Example of Neisseria meningitidis. Front Cell Infect Microbiol, 3(102).

Harrison, L. H., Dwyer, D. M., Maples, C. T., \& Billmann, L. (1999). Risk of meningococcal infection in college students. Jama, 281(20), 1906-1910.

Harrison, L. H., Trotter, C. L., \& Ramsay, M. E. (2009). Global epidemiology of meningococcal disease. Vaccine, 27 Suppl 2, B51-63.

Hart, C. A., \& Thomson, A. P. J. (2006). Meningococcal disease and its management in children. BMJ, 333(7570), 685-690. 
Hase, R., Hosokawa, N., Yaegashi, M., \& Muranaka, K. (2014). Bacterial meningitis in the absence of cerebrospinal fluid pleocytosis: A case report and review of the literature. Can J Infect Dis Med Microbiol, 25(5), 249-251.

Hawkins, B. T., \& Egleton, R. D. (2006). Fluorescence imaging of blood-brain barrier disruption. J Neurosci Methods, 151(2), 262-267.

Henderson, B., \& Martin, A. (2011). Bacterial Virulence in the Moonlight: Multitasking Bacterial Moonlighting Proteins Are Virulence Determinants in Infectious Disease. Infect Immun, 79(9), 3476.

Henderson, I. R., Owen, P., \& Nataro, J. P. (1999). Molecular switches - the ON and OFF of bacterial phase variation. Mol Microbiol, 33(5), 919-932.

Henriques-Normark, B., \& Normark, S. (2010). Commensal pathogens, with a focus on Streptococcus pneumoniae, and interactions with the human host. Exp Cell Res, 316(8), 1408-1414.

Hill, D. J., Griffiths, N. J., Borodina, E., \& Virji, M. (2010). Cellular and molecular biology of Neisseria meningitidis colonization and invasive disease. Clin Sci (Lond), 118(9), 547-564.

Holbein, B. E. (1981). Enhancement of Neisseria meningitidis infection in mice by addition of iron bound to transferrin. Infect Immun, 34(1), 120-125.

Hood, M. I., \& Skaar, E. P. (2012). Nutritional immunity: transition metals at the pathogen-host interface. Nat Rev Microbiol, 10(8), 525-537.

Horton, R. E., Stuart, J., Christensen, H., Borrow, R., Guthrie, T., Davenport, V., Finn, A., Williams, N. A., \& Heyderman, R. S. (2005). Influence of age and carriage status on salivary IgA to Neisseria meningitidis. Epidemiol Infect, 133(5), 883-889.

Huberts, D. H. E. W., \& van der Klei, I. J. (2010). Moonlighting proteins: An intriguing mode of multitasking. Biochim Biophys Acta, 1803(4), 520-525.

Hung, M. C., \& Christodoulides, M. (2013). The biology of Neisseria adhesins. Biology, 2(3), 1054-1109.

Imrey, P. B., Jackson, L. A., Ludwinski, P. H., England, A. C., 3rd, Fella, G. A., Fox, B. C., Isdale, L. B., Reeves, M. W., \& Wenger, J. D. (1996). Outbreak of serogroup C meningococcal disease associated with campus bar patronage. Am J Epidemiol, 143(6), 624-630.

Jafri, R. Z., Ali, A., Messonnier, N. E., Tevi-Benissan, C., Durrheim, D., Eskola, J., Fermon, F., Klugman, K. P., Ramsay, M., Sow, S., Zhujun, S., Bhutta, Z. A., \& Abramson, J. (2013). Global epidemiology of invasive meningococcal disease. Popul Health Metr, 11(1), 17. 
Jeney, V., Balla, G., \& Balla, J. (2014). Red blood cell, hemoglobin and heme in the progression of atherosclerosis. Frontiers in physiology, 5(379).

Jerse, A. E., Bash, M. C., \& Russell, M. W. (2014). Vaccines against gonorrhea: current status and future challenges. Vaccine, 32(14), 1579-1587.

Johansson, L., Rytkonen, A., Bergman, P., Albiger, B., Kallstrom, H., Hokfelt, T., Agerberth, B., Cattaneo, R., \& Jonsson, A. B. (2003). CD46 in meningococcal disease. Science, 301(5631), 373-375.

Johnson, D. C., Dean, D. R., Smith, A. D., \& Johnson, M. K. (2005). Structure, function, and formation of biological iron-sulfur clusters. Annu Rev Biochem, 74, 247-281.

Johswich, K. O., Zhou, J., Law, D. K., St Michael, F., McCaw, S. E., Jamieson, F. B., Cox, A. D., Tsang, R. S., \& Gray-Owen, S. D. (2012). Invasive potential of nonencapsulated disease isolates of Neisseria meningitidis. Infect Immun, 80(7), 2346-2353.

Jolley, K. A., Brehony, C., \& Maiden, M. C. J. (2007). Molecular typing of meningococci: recommendations for target choice and nomenclature. FEMS Microbiol Rev, 31(1), 8996.

Jolley, K. A., \& Maiden, M. C. (2014). Using multilocus sequence typing to study bacterial variation: prospects in the genomic era. Future Microbiol, 9(5), 623-630.

Jordan, P. W., \& Saunders, N. J. (2009). Host iron binding proteins acting as niche indicators for Neisseria meningitidis. Plos One, 4(4), e5198-e5198.

Joseph, B., Schneiker-Bekel, S., Schramm-Glück, A., Blom, J., Claus, H., Linke, B., Schwarz, R. F., Becker, A., Goesmann, A., Frosch, M., \& Schoen, C. (2010). Comparative Genome Biology of a Serogroup B Carriage and Disease Strain Supports a Polygenic Nature of Meningococcal Virulence. J Bacteriol, 192(20), 5363.

Kallstrom, H., Islam, M. S., Berggren, P. O., \& Jonsson, A. B. (1998). Cell Signaling by the Type IV Pili of Pathogenic Neisseria. 273(34), 21777-21782.

Katona, P., \& Katona-Apte, J. (2008). The Interaction between Nutrition and Infection. Clin Infect Dis, 46(10), 1582-1588.

Kaur, K., Gupta, R., Saraf, S. A., \& Saraf, S. K. (2014). Zinc: the metal of life. Compr Rev Food Sci Food Saf, 13(4), 358-376.

Kelly, C., Arnold, R., Galloway, Y., \& O'Hallahan, J. (2007). A prospective study of the effectiveness of the New Zealand meningococcal B vaccine. Am J Epidemiol, 166(7), 817823. 
Kesici, U., Kesici, S., Ulusoy, H., Yucesan, F., Turkmen, A. U., Besir, A., \& Tuna, V. (2015). Effects of glutamine on wound healing. Int Wound J, 12(3), 280-284.

Kheradmand, F., Folkesson, H. G., Shum, L., Derynk, R., Pytela, R., \& Matthay, M. A. (1994). Transforming growth factor-alpha enhances alveolar epithelial cell repair in a new in vitro model. Am J Physiol, 267(6 Pt 1), L728-738.

Kiefer, F. (1896). Zur Differentialdiagnose des Erregers der epidemischen Cerebrospinalmeningitidis und der Gonorrhoe. Berl Klin Woch, 33, 628-630.

Klemm, P., \& Schembri, M. A. (2000). Bacterial adhesins: function and structure. Int J Med Microbiol, 290(1), 27-35.

Kline, K. A., Sechman, E. V., Skaar, E. P., \& Seifert, H. S. (2003). Recombination, repair and replication in the pathogenic Neisseriae: the 3 R's of molecular genetics of two humanspecific bacterial pathogens. Mol Microbiol, 50(1), 3-13.

Klughammer, J., Dittrich, M., Blom, J., Mitesser, V., Vogel, U., Frosch, M., Goesmann, A., Müller, T., \& Schoen, C. (2017). Comparative Genome Sequencing Reveals Within-Host Genetic Changes in Neisseria meningitidis during Invasive Disease. Plos One, 12(1), e0169892.

Klumpp, S., \& Hwa, T. (2014). Bacterial growth: global effects on gene expression, growth feedback and proteome partition. Current opinion in biotechnology, 28, 96-102.

Kojima, T., Go, M., Takano, K., Kurose, M., Ohkuni, T., Koizumi, J., Kamekura, R., Ogasawara, N., Masaki, T., Fuchimoto, J., Obata, K., Hirakawa, S., Nomura, K., Keira, T., Miyata, R., Fujii, N., Tsutsumi, H., Himi, T., \& Sawada, N. (2013). Regulation of tight junctions in upper airway epithelium. Biomed Res Int, 2013, 947072.

Krachler, A. M., \& Orth, K. (2013). Targeting the bacteria-host interface: strategies in antiadhesion therapy. Virulence, 4(4), 284-294.

Kristiansen, M., Graversen, J. H., Jacobsen, C., Sonne, O., Hoffman, H. J., Law, S. K., \& Moestrup, S. K. (2001). Identification of the haemoglobin scavenger receptor. Nature, 409(6817), 198-201.

Lambotin, M., Hoffmann, I., Laran-Chich, M., Nassif, X., Couraud, P. O., \& Bourdoulous, S. (2005). Invasion of endothelial cells by Neisseria meningitidis requires cortactin recruitment by a phosphoinositide-3-kinase/Rac1 signalling pathway triggered by the lipo-oligosaccharide. Journal of Cell Science, 118(16), 3805.

Langmead, B., \& Salzberg, S. L. (2012). Fast gapped-read alignment with Bowtie 2. Nat Methods, 9(4), 357-359. 
Lansdown, A. B. G., Mirastschijski, U., Stubbs, N., Scanlon, E., \& Aagren, M. S. (2007). Zinc in wound healing: Theoretical, experimental, and clinical aspects. Wound Repair Regen, 15(1), 2-16.

Larson, J. A., Howie, H. L., \& So, M. (2004). Neisseria meningitidis accelerates ferritin degradation in host epithelial cells to yield an essential iron source. Mol Microbiol, 53(3), 807-820.

Lee, B., \& Hill, P. (1993). Identification of an outer-membrane haemoglobin-binding protein in Neisseria meningitidis (Vol. 138).

Leoni, G., Neumann, P. A., Sumagin, R., Denning, T. L., \& Nusrat, A. (2015). Wound repair: role of immune-epithelial interactions. Mucosal immunology, 8(5), 959-968.

Lewis, L. A., \& Dyer, D. W. (1995). Identification of an iron-regulated outer membrane protein of Neisseria meningitidis involved in the utilization of hemoglobin complexed to haptoglobin. J Bacteriol, 177(5), 1299-1306.

Lewis, L. A., Gipson, M., Hartman, K., Ownbey, T., Vaughn, J., \& Dyer, D. W. (1999). Phase variation of $\mathrm{HpuAB}$ and $\mathrm{HmbR}$, two distinct haemoglobin receptors of Neisseria meningitidis DNM2. Mol Microbiol, 32(5), 977-989.

Lewis, L. A., Gray, E., Wang, Y. P., Roe, B. A., \& Dyer, D. W. (1997). Molecular characterization of hpuAB, the haemoglobin-haptoglobin-utilization operon of Neisseria meningitidis. Mol Microbiol, 23(4), 737-749.

Lim, S. K., Kim, H., Lim, S. K., bin Ali, A., Lim, Y. K., Wang, Y., Chong, S. M., Costantini, F., \& Baumman, H. (1998). Increased susceptibility in Hp knockout mice during acute hemolysis. Blood, 92(6), 1870-1877.

Lim, S. Y., Yuzhalin, A. E., Gordon-Weeks, A. N., \& Muschel, R. J. (2016). Targeting the CCL2CCR2 signaling axis in cancer metastasis. Oncotarget, 7(19), 28697-28710.

Lipsitch, M., \& Moxon, E. R. (1997). Virulence and transmissibility of pathogens: what is the relationship? Trends Microbiol, 5(1), 31-37.

Lonnerdal, B., \& lyer, S. (1995). Lactoferrin: molecular structure and biological function. Annu Rev Nutr, 15, 93-110.

Lopez, L., \& Sherwood, J. (2014). The Epidemiology of Meningococcal Disease In New Zealand. Institute of Environmental Science and Research Ltd (ESR), Wellington, New Zealand.

Lucidarme, J., Comanducci, M., Findlow, J., Gray, S. J., Kaczmarski, E. B., Guiver, M., Vallely, P. J., Oster, P., Pizza, M., Bambini, S., Muzzi, A., \& Borrow, R. (2010). Characterization of $f H b p$, nhba (gna2132), nadA, porA, and Sequence Type in Group B Meningococcal Case Isolates Collected in England and Wales during January 2008 and Potential Coverage of 
an Investigational Group B Meningococcal Vaccine. Clin Vaccine Immunol, 17(6), 919929.

Maffei, M., Funicello, M., Vottari, T., Gamucci, O., Costa, M., Lisi, S., Viegi, A., Ciampi, O., Bardi, G., Vitti, P., Pinchera, A., \& Santini, F. (2009). The obesity and inflammatory marker haptoglobin attracts monocytes via interaction with chemokine (C-C motif) receptor 2 (CCR2). BMC Biology, 7(1), 87.

Maiden, M. C., Bygraves, J. A., Feil, E., Morelli, G., Russell, J. E., Urwin, R., Zhang, Q., Zhou, J., Zurth, K., Caugant, D. A., Feavers, I. M., Achtman, M., \& Spratt, B. G. (1998). Multilocus sequence typing: a portable approach to the identification of clones within populations of pathogenic microorganisms. Proc Natl Acad Sci U S A, 95(6), 3140-3145.

Manso, C., Rivas, I., Peraire, J., Vidal, F., \& Richart, C. (1997). Fatal Listeria meningitis, endocarditis and pericarditis in a patient with haemochromatosis. Scand J Infect Dis, 29(3), 308-309.

Marchiafava, E., \& Celli, A. (1884). Spra i micrococchi della meningite cerebrospinale epidemica. Gazz degli Ospedali, 5, 59.

Martin, D., \& Lopez, L. (2009). The Epidemiology of Meningococcal Disease in New Zealand in 2008 Institute of Environmental Science and Research Ltd (ESR).

Martiny, N., \& Chiapello, I. (2013). Assessments for the impact of mineral dust on the meningitis incidence in West Africa. Atmos Environ, 70, 245-253.

McKenna, A., Hanna, M., Banks, E., Sivachenko, A., Cibulskis, K., Kernytsky, A., Garimella, K., Altshuler, D., Gabriel, S., Daly, M., \& DePristo, M. A. (2010). The Genome Analysis Toolkit: a MapReduce framework for analyzing next-generation DNA sequencing data. Genome Res, 20(9), 1297-1303.

Mehta, N., \& Levin, M. (2000). Management and prevention of meningococcal disease. Hosp Pract (1995), 35(8), 75-86.

Merz, A. J., \& So, M. (2000). Interactions of pathogenic neisseriae with epithelial cell membranes. Annu Rev Cell Dev Biol, 16, 423-457.

Meyers, L. A., Levin, B. R., Richardson, A. R., \& Stojiljkovic, I. (2003). Epidemiology, hypermutation, within-host evolution and the virulence of Neisseria meningitidis. Proc Biol Sci, 270(1525), 1667-1677.

Mietzner, T. A., \& Morse, S. A. (2010). Neisseria Topley \& Wilson's Microbiology and Microbial Infections: John Wiley \& Sons, Ltd.

$\mathrm{MOH}$. (2018). Meningococcal disease: Information for general practitioners and emergency departments. New Zealand: Ministry of Health. 
Montanari, P., Bozza, G., Capecchi, B., Caproni, E., Barrile, R., Norais, N., Capitani, M., Sallese, M., Cecchini, P., Ciucchi, L., Gao, Z. N., Rappuoli, R., Pizza, M., Arico, B., \& Merola, M. (2012). Human heat shock protein (Hsp) 90 interferes with Neisseria meningitidis adhesin A (NadA)-mediated adhesion and invasion. Cell Microbiol, 14(3), 368-385.

Moxon, E. R., \& Kroll, J. S. (1990). The role of bacterial polysaccharide capsules as virulence factors. Curr Top Microbiol Immunol, 150, 65-85.

Mueller, J. E., \& Gessner, B. D. (2010). A hypothetical explanatory model for meningococcal meningitis in the African meningitis belt. Int J Infect Dis, 14(7), E553-E559.

Nagele, V., Heesemann, J., Schielke, S., Jimenez-Soto, L. F., Kurzai, O., \& Ackermann, N. (2011). Neisseria meningitidis Adhesin NadA Targets beta 1 Integrins Functional similarity to Yersinia invasin. J Biol Chem, 286(23), 20536-20546.

Nassif, X., Lowy, J., Stenberg, P., O'Gaora, P., Ganji, A., \& So, M. (1993). Antigenic variation of pilin regulates adhesion of Neisseria meningitidis to human epithelial cells. $\mathrm{Mol}$ Microbiol, 8(4), 719-725.

Nield, L. S., \& Weisse, M. E. (2008). Treatment and prevention of $N$. meningitidis infection. Infections in medicine, 25(3), 128.

Nielsen, M. J., \& Moestrup, S. K. (2009). Receptor targeting of hemoglobin mediated by the haptoglobins: roles beyond heme scavenging. Blood, 114(4), 764.

Noinaj, N., Easley, N. C., Oke, M., Mizuno, N., Gumbart, J., Boura, E., Steere, A. N., Zak, O., Aisen, P., Tajkhorshid, E., Evans, R. W., Gorringe, A. R., Mason, A. B., Steven, A. C., \& Buchanan, S. K. (2012). Structural basis for iron piracy by pathogenic Neisseria. Nature, 483(7387), 53-58.

Norrander, J., Kempe, T., \& Messing, J. (1983). Construction of improved M13 vectors using oligodeoxynucleotide-directed mutagenesis. Gene, 26(1), 101-106.

O'Keefe, G. E., Hybki, D. L., \& Munford, R. S. (2002). The G-->A single nucleotide polymorphism at the -308 position in the tumor necrosis factor-alpha promoter increases the risk for severe sepsis after trauma. J Trauma, 52(5), 817-826.

Ofek, I., Hasty, D. L., \& Sharon, N. (2003). Anti-adhesion therapy of bacterial diseases: prospects and problems. Pathog Dis, 38(3), 181-191.

Ohalloran, T. V. (1993). Transition metals in control of gene expression. Science, 261(5122), 715-725.

Omer, H., Rose, G., Jolley, K. A., Frapy, E., Zahar, J. R., Maiden, M. C. J., Bentley, S. D., Tinsley, C. R., Nassif, X., \& Bille, E. (2011). Genotypic and Phenotypic Modifications of Neisseria meningitidis after an Accidental Human Passage. Plos One, 6(2), e17145. 
Pace, D., \& Pollard, A. J. (2012). Meningococcal disease: clinical presentation and sequelae. Vaccine, 30 Suppl 2, B3-9.

Pandey, A., Bringel, F., \& Meyer, J. M. (1994). Iron requirement and search for siderophores in lactic acid bacteria. Appl Microbiol Biotechnol, 40, 735-739.

Parker, D., \& Prince, A. (2011). Innate immunity in the respiratory epithelium. Am J Respir Cell Mol Biol, 45(2), 189-201.

Pentecost, M., Otto, G., Theriot, J. A., \& Amieva, M. R. (2006). Listeria monocytogenes Invades the Epithelial Junctions at Sites of Cell Extrusion. PLOS Pathog, 2(1), e3.

Perkins-Balding, D., Ratliff-Griffin, M., \& Stojiljkovic, I. (2004). Iron transport systems in Neisseria meningitidis. Microbiol Mol Biol Rev, 68(1), 154-171.

Perrio, M. J., Ewen, D., Trevethick, M. A., Salmon, G. P., \& Shute, J. K. (2007). Fibrin formation by wounded bronchial epithelial cell layers in vitro is essential for normal epithelial repair and independent of plasma proteins. Clin Exp Allergy, 37(11), 1688-1700.

Petousis-Harris, H., Paynter, J., Morgan, J., Saxton, P., McArdle, B., Goodyear-Smith, F., \& Black, S. (2017). Effectiveness of a group $B$ outer membrane vesicle meningococcal vaccine against gonorrhoea in New Zealand: a retrospective case-control study. Lancet, 390(10102), 1603-1610.

Pettersson, A., Maas, A., van Wassenaar, D., van der Ley, P., \& Tommassen, J. (1995). Molecular characterization of $\mathrm{FrpB}$, the 70-kilodalton iron-regulated outer membrane protein of Neisseria meningitidis. Infect Immun, 63(10), 4181-4184.

Piva, M., Horowitz, G. M., \& Sharpe-Timms, K. L. (2001). Interleukin-6 differentially stimulates haptoglobin production by peritoneal and endometriotic cells in vitro: a model for endometrial-peritoneal interaction in endometriosis. J Clin Endocrinol Metab, 86(6), 2553-2561.

Pizarro-Cerda, J., \& Cossart, P. (2006). Bacterial adhesion and entry into host cells. Cell, 124(4), 715-727.

Porcheron, G., Garenaux, A., Proulx, J., Sabri, M., \& Dozois, C. M. (2013). Iron, copper, zinc and manganese transport and regulation in pathogenic Enterobacteria: correlations between strains, site of infection and the relative importance of the different metal transport systems for virulence. Front Cell Infect Microbiol, 3(90).

Posey, J. E., \& Gherardini, F. C. (2000). Lack of a role for iron in the Lyme disease pathogen. Science, 288(5471), 1651-1653.

Price, M. N., Wetmore, K. M., Deutschbauer, A. M., \& Arkin, A. P. (2016). A Comparison of the Costs and Benefits of Bacterial Gene Expression. Plos One, 11(10), e0164314-e0164314. 
Quaye, I. K. (2015). Extracellular hemoglobin: the case of a friend turned foe. Frontiers in physiology, 6, 96-96.

R Core Development Team. (2014). R: A Language and Environment for Statistical Computing. Vienna, Austria: R Foundation for Statistical Computing.

Ragunathan, P., Sridaran, D., Weigel, A., Shabayek, S., Spellerberg, B., \& Ponnuraj, K. (2013). Metal Binding Is Critical for the Folding and Function of Laminin Binding Protein, Lmb of Streptococcus agalactiae. Plos One, 8(6), e67517.

Rahman, I., Biswas, S. K., Jimenez, L. A., Torres, M., \& Forman, H. J. (2005). Glutathione, stress responses, and redox signaling in lung inflammation. Antioxid Redox Signal, 7(1-2), 4259.

Ratledge, C., \& Dover, L. G. (2000). Iron metabolism in pathogenic bacteria. Annu Rev Microbiol, $54,881-941$.

Raymond, K. N., Dertz, E. A., \& Kim, S. S. (2003). Enterobactin: An archetype for microbial iron transport. PNAS, 100(7), 3584-3588.

Read, R. C., Teare, D. M., Pridmore, A. C., Naylor, S. C., Timms, J. M., Kaczmarski, E. B., Borrow, R., \& Wilson, A. G. (2009). The tumor necrosis factor polymorphism TNF (-308) is associated with susceptibility to meningococcal sepsis, but not with lethality. Crit Care Med, 37(4), 1237-1243.

Reisinger, K. S., Baxter, R., Block, S. L., Shah, J., Bedell, L., \& Dull, P. M. (2009). Quadrivalent meningococcal vaccination of adults: phase III comparison of an investigational conjugate vaccine, MenACWY-CRM, with the licensed vaccine, Menactra. Clin Vaccine Immunol, 16(12), 1810-1815.

Ren, X., Eccles, D. A., Greig, G. A., Clapham, J., Wheeler, N. E., Lindgreen, S., Gardner, P. P., \& MacKichan, J. K. (2017). Genomic, Transcriptomic, and Phenotypic Analyses of Neisseria meningitidis Isolates from Disease Patients and Their Household Contacts. mSystems, 2(6).

Ren, X., \& MacKichan, J. K. (2014). Disease-associated Neisseria meningitidis isolates inhibit wound repair in respiratory epithelial cells in a type IV pilus-independent manner. Infect Immun, 82(12), 5023-5034.

Rodríguez-Arce, I., Al-Jubair, T., Euba, B., Fernández-Calvet, A., Gil-Campillo, C., Martí, S., Törnroth-Horsefield, S., Riesbeck, K., \& Garmendia, J. (2019). Moonlighting of Haemophilus influenzae heme acquisition systems contributes to the host airwaypathogen interplay in a coordinated manner. Virulence, 10(1), 315-333. 
Rohde, K. H., \& Dyer, D. W. (2004). Analysis of Haptoglobin and Hemoglobin-Haptoglobin Interactions with the Neisseria meningitidis TonB-Dependent Receptor HpuAB by Flow Cytometry. Infect Immun, 72(5), 2494-2506.

Rohmer, L., Hocquet, D., \& Miller, S. I. (2011). Are pathogenic bacteria just looking for food? Metabolism and microbial pathogenesis. Trends Microbiol, 19(7), 341-348.

Rosenstein, N. E., Perkins, B. A., Stephens, D. S., Popovic, T., \& Hughes, J. M. (2001). Meningococcal disease. N Engl J Med, 344(18), 1378-1388.

Rouphael, N. G., \& Stephens, D. S. (2012). Neisseria meningitidis: biology, microbiology, and epidemiology. Methods Mol Biol, 799, 1-20.

Rozen, S., \& Skaletsky, H. (2000). Primer3 on the WWW for general users and for biologist programmers. Methods Mol Biol, 132, 365-386.

Rutz, J. M., Abdullah, T., Singh, S. P., Kalve, V. I., \& Klebba, P. E. (1991). Evolution of the ferric enterobactin receptor in gram-negative bacteria. J Bacteriol, 173(19), 5964-5974.

SAS. (2013). SAS 9.4. Cary, NC, USA: SAS Institute Inc.

Scarselli, M., Serruto, D., Montanari, P., Capecchi, B., Adu-Bobie, J., Veggi, D., Rappuoli, R., Pizza, M., \& Arico, B. (2006). Neisseria meningitidis NhhA is a multifunctional trimeric autotransporter adhesin. Mol Microbiol, 61(3), 631-644.

Schaer, D. J., Vinchi, F., Ingoglia, G., Tolosano, E., \& Buehler, P. W. (2014). Haptoglobin, hemopexin, and related defense pathways-basic science, clinical perspectives, and drug development. Frontiers in physiology, 5(415).

Schafer, F. Q., Qian, S. Y., \& Buettner, G. R. (2000). Iron and free radical oxidations in cell membranes. Cellular Mol Biol (Noisy-le-Grand), 46(3), 657-662.

Schalk, I. J. (2008). Metal trafficking via siderophores in Gram-negative bacteria: specificities and characteristics of the pyoverdine pathway. J Inorg Biochem, 102(5-6), 1159-1169.

Schneider, C. A., Rasband, W. S., \& Eliceiri, K. W. (2012). NIH Image to ImageJ: 25 years of image analysis. Nature Methods, 9(7), 671-675.

Schoen, C., Blom, J., Claus, H., Schramm-Gluck, A., Brandt, P., Muller, T., Goesmann, A., Joseph, B., Konietzny, S., Kurzai, O., Schmitt, C., Friedrich, T., Linke, B., Vogel, U., \& Frosch, M. (2008). Whole-genome comparison of disease and carriage strains provides insights into virulence evolution in Neisseria meningitidis. PNAS, 105(9), 3473-3478.

Schoen, C., Kischkies, L., Elias, J., \& Ampattu, B. J. (2014). Metabolism and virulence in Neisseria meningitidis. Front Cell Infect Microbiol, 4, 114. 
Schoen, C., Tettelin, H., Parkhill, J., \& Frosch, M. (2009). Genome flexibility in Neisseria meningitidis. Vaccine, 27, B103-B111.

Scott, K. A., Arnott, C. H., Robinson, S. C., Moore, R. J., Thompson, R. G., Marshall, J. F., \& Balkwill, F. R. (2004). TNF-alpha regulates epithelial expression of MMP-9 and integrin alphavbeta 6 during tumour promotion. A role for TNF-alpha in keratinocyte migration? Oncogene, 23(41), 6954-6966.

Semchenko, E. A., Tan, A., Borrow, R., \& Seib, K. L. (2018). The serogroup B meningococcal vaccine Bexsero elicits antibodies to Neisseria gonorrhoeae. Clin Infect Dis.

Shaik, Y. B., Grogan, S., Davey, M., Sebastian, S., Goswami, S., Szmigielski, B., \& Genco, C. A. (2007). Expression of the Iron-Activated $n s p A$ and secY Genes in Neisseria meningitidis Group B by Fur-Dependent and -Independent Mechanisms. J Bacteriol, 189(2), 663.

Shelburne, S. A., Davenport, M. T., Keith, D. B., \& Musser, J. M. (2008). The role of complex carbohydrate catabolism in the pathogenesis of invasive streptococci. Trends Microbiol, 16(7), 318-325.

Shih, A. W. Y., McFarlane, A., \& Verhovsek, M. (2014). Haptoglobin testing in hemolysis: Measurement and interpretation. Am J Hematol, 89(4), 443-447.

Sim, R. J., Harrison, M. M., Moxon, E. R., \& Tang, C. M. (2000). Underestimation of meningococci in tonsillar tissue by nasopharyngeal swabbing. Lancet, 356(9242), 16531654.

Simmons, G., Martin, D., Stewart, J., Jones, N., Calder, L., \& Bremner, D. (2001). Carriage of Neisseria meningitidis among household contacts of patients with meningococcal disease in New Zealand. Eur J Clin Microbiol Infect Dis, 20(4), 237-242.

Singh, B., Al-Jubair, T., Morgelin, M., Thunnissen, M. M., \& Riesbeck, K. (2013). The unique structure of Haemophilus influenzae protein E reveals multiple binding sites for host factors. Infect Immun, 81(3), 801-814.

Sjolinder, H., \& Jonsson, A. B. (2010). Olfactory nerve--a novel invasion route of Neisseria meningitidis to reach the meninges. Plos One, 5(11), e14034.

Skaar, E. P. (2010). The battle for iron between bacterial pathogens and their vertebrate hosts. Plos Pathogens, 6(8), e1000949-e1000949.

Snyder, L. A. S., \& Saunders, N. J. (2006). The majority of genes in the pathogenic Neisseria species are present in non-pathogenic Neisseria lactamica, including those designated as 'virulence genes'. BMC Genomics, 7, 128.

Spellerberg, B., Rozdzinski, E., Martin, S., Weber-Heynemann, J., Schnitzler, N., Lütticken, R., \& Podbielski, A. (1999). Lmb, a Protein with Similarities to the Lral Adhesin Family, 
Mediates Attachment of Streptococcus agalactiae to Human Laminin. Infect Immun, $67(2), 871$.

Spence, J. M., Wright, L., \& Clark, V. L. (2008). Laboratory maintenance of Neisseria gonorrhoeae. Curr Protoc Microbiol, Chapter 4, Unit 4A.1.

Spencer-Smith, R., Roberts, S., Gurung, N., \& Snyder, L. A. S. (2016). DNA uptake sequences in Neisseria gonorrhoeae as intrinsic transcriptional terminators and markers of horizontal gene transfer. Microbial genomics, 2(8), e000069-e000069.

Spratt, B. G. (1999). Multilocus sequence typing: molecular typing of bacterial pathogens in an era of rapid DNA sequencing and the internet. Curr Opin Microbiol, 2(3), 312-316.

Stephens, D. S. (1999). Uncloaking the meningococcus: dynamics of carriage and disease. Lancet, 353(9157), 941-942.

Stephens, D. S. (2009). Biology and pathogenesis of the evolutionarily successful, obligate human bacterium Neisseria meningitidis. Vaccine, 27, B71-B77.

Stephens, D. S., \& Farley, M. M. (1991). Pathogenic events during infection of the human nasopharynx with Neisseria meningitidis and Haemophilus influenzae. Rev Infect Dis, 13(1), 22-33.

Stephens, D. S., Greenwood, B., \& Brandtzaeg, P. (2007). Epidemic meningitis, meningococcaemia, and Neisseria meningitidis. Lancet, 369(9580), 2196-2210.

Stephens, D. S., Hoffman, L. H., \& McGee, Z. A. (1983). Interaction of Neisseria meningitidis with human nasopharyngeal mucosa - attachment and entry into columnar epithelial cells. $J$ Infect Dis, 148(3), 369-376.

Stojiljkovic, I., Hwa, V., de Saint Martin, L., O'Gaora, P., Nassif, X., Heffron, F., \& So, M. (1995). The Neisseria meningitidis haemoglobin receptor: its role in iron utilization and virulence. Mol Microbiol, 15(3), 531-541.

Stojiljkovic, I., Larson, J., Hwa, V., Anic, S., \& So, M. (1996). HmbR outer membrane receptors of pathogenic Neisseria spp.: iron-regulated, hemoglobin-binding proteins with a high level of primary structure conservation. J Bacteriol, 178(15), 4670-4678.

Stork, M., Bos, M. P., Jongerius, I., de Kok, N., Schilders, I., Weynants, V. E., Poolman, J. T., \& Tommassen, J. (2010). An Outer Membrane Receptor of Neisseria meningitidis Involved in Zinc Acquisition with Vaccine Potential. Plos Pathogens, 6(7), e1000969.

Stork, M., Grijpstra, J., Bos, M. P., Torres, C. M., Devos, N., Poolman, J. T., Chazin, W. J., \& Tommassen, J. (2013). Zinc Piracy as a Mechanism of Neisseria meningitidis for Evasion of Nutritional Immunity. Plos Pathogens, 9(10), 10. 
Sultan, B., Labadi, K., Guegan, J. F., \& Janicot, S. (2005). Climate drives the meningitis epidemics onset in West Africa. Plos Medicine, 2(1), 43-49.

Sun, X., Zhou, H., Xu, L., Yang, H., Gao, Y., Zhu, B., \& Shao, Z. (2013). Prevalence and genetic diversity of two adhesion-related genes, pilE and nadA, in Neisseria meningitidis in China. Epidemiol Infect, 141(10), 2163-2172.

Swain, C. L., \& Martin, D. R. (2007). Survival of meningococci outside of the host: implications for acquisition. Epidemiol Infect, 135(2), 315-320.

Swanson, J., Belland, R. J., \& Hill, S. A. (1992). Neisserial surface variation: how and why? Curr Opin Genet Dev, 2(5), 805-811.

Tan, L. K. K., Carlone, G. M., \& Borrow, R. (2010). Advances in the Development of Vaccines against Neisseria meningitidis. N Engl J Med, 362(16), 1511-1520.

Tapsall, J. W., Ndowa, F., Lewis, D. A., \& Unemo, M. (2009). Meeting the public health challenge of multidrug- and extensively drug-resistant Neisseria gonorrhoeae. Expert Rev Anti Infect Ther, 7(7), 821-834.

Tauseef, I., Harrison, O. B., Wooldridge, K. G., Feavers, I. M., Neal, K. R., Gray, S. J., Kriz, P., Turner, D. P. J., Ala'Aldeen, D. A. A., Maiden, M. C. J., \& Bayliss, C. D. (2011). Influence of the combination and phase variation status of the haemoglobin receptors $\mathrm{HmbR}$ and HpuAB on meningococcal virulence. Microbiology, 157(Pt 5), 1446-1456.

Tavano, R., Capecchi, B., Montanari, P., Franzoso, S., Marin, O., Sztukowska, M., Cecchini, P., Segat, D., Scarselli, M., Arico, B., \& Papini, E. (2011). Mapping of the Neisseria meningitidis NadA Cell-Binding Site: Relevance of Predicted alpha-Helices in the NH2Terminal and Dimeric Coiled-Coil Regions. J Bacteriol, 193(1), 107-115.

Theilgaard-Mönch, K., Jacobsen, L. C., Nielsen, M. J., Rasmussen, T., Udby, L., Gharib, M., Arkwright, P. D., Gombart, A. F., Calafat, J., Moestrup, S. K., Porse, B. T., \& Borregaard, N. (2006). Haptoglobin is synthesized during granulocyte differentiation, stored in specific granules, and released by neutrophils in response to activation. Blood, 108(1), 353.

Thompson, C., \& Fuhrman, M. P. (2005). Nutrients and Wound Healing: Still Searching for the Magic Bullet. Nutr Clin Pract, 20(3), 331-347.

Thompson, E. A., Feavers, I. M., \& Maiden, M. C. (2003). Antigenic diversity of meningococcal enterobactin receptor FetA, a vaccine component. Microbiology, 149(Pt 7), 1849-1858.

Thompson, J. D., Higgins, D. G., \& Gibson, T. J. (1994). CLUSTAL W: improving the sensitivity of progressive multiple sequence alignment through sequence weighting, position-specific gap penalties and weight matrix choice. Nucleic Acids Res, 22(22), 4673-4680. 
Thompson, M. J., Ninis, N., Perera, R., Mayon-White, R., Phillips, C., Bailey, L., Harnden, A., Mant, D., \& Levin, M. (2006). Clinical recognition of meningococcal disease in children and adolescents. Lancet, 367(9508), 397-403.

Torti, F. M., \& Torti, S. V. (2002). Regulation of ferritin genes and protein. Blood, 99(10), 3505.

Townsend, R., Goodwin, L., Stevanin, T. M., Silcocks, P. B., Parker, A., Maiden, M. C. J., \& Read, R. C. (2002). Invasion by Neisseria meningitidis varies widely between clones and among nasopharyngeal mucosae derived from adult human hosts. Microbiology, 148, 14671474.

Trivedi, K., Tang, C. M., \& Exley, R. M. (2011). Mechanisms of meningococcal colonisation. Trends Microbiol, 19(9), 456-463.

Trotter, C. L., \& Greenwood, B. M. (2007). Meningococcal carriage in the African meningitis belt. Lancet Infect Dis, 7(12), 797-803.

Trotter, C. L., Lingani, C., Fernandez, K., Cooper, L. V., Bita, A., Tevi-Benissan, C., Ronveaux, O., Préziosi, M. P., \& Stuart, J. M. (2017). Impact of MenAfriVac in nine countries of the African meningitis belt, 2010-15: an analysis of surveillance data. Lancet Infect Dis, 17(8), 867-872.

Troxell, B., \& Hassan, H. M. (2013). Transcriptional regulation by Ferric Uptake Regulator (Fur) in pathogenic bacteria. Front Cell Infect Microbiol, 3, 59.

Tseng, C. F., Lin, C. C., Huang, H. Y., Liu, H. C., \& Mao, S. J. (2004). Antioxidant role of human haptoglobin. Proteomics, 4(8), 2221-2228.

Tully, J., Viner, R. M., Coen, P. G., Stuart, J. M., Zambon, M., Peckham, C., Booth, C., Klein, N., Kaczmarski, E., \& Booy, R. (2006). Risk and protective factors for meningococcal disease in adolescents: matched cohort study. BMJ, 332(7539), 445.

Tzeng, Y. L., \& Stephens, D. S. (2000). Epidemiology and pathogenesis of Neisseria meningitidis. Microb Infect, 2(6), 687-700.

Unemo, M., \& Shafer, W. M. (2014). Antimicrobial Resistance in Neisseria gonorrhoea in the 21st Century: Past, Evolution, and Future. Clin Microbiol Rev, 27(3), 587.

UniProt, C. (2008). The universal protein resource (UniProt). Nucleic acids research, 36(Database issue), D190-D195.

van de Beek, D., de Gans, J., Spanjaard, L., Weisfelt, M., Reitsma, J. B., \& Vermeulen, M. (2004). Clinical features and prognostic factors in adults with bacterial meningitis. N Engl J Med, 351(18), 1849-1859. 
van der Woude, M. W., \& Bäumler, A. J. (2004). Phase and antigenic variation in bacteria. Clin Microbiol Rev, 17(3), 581-611.

van Deuren, M., Brandtzaeg, P., \& van der Meer, J. W. (2000). Update on meningococcal disease with emphasis on pathogenesis and clinical management. Clin Microbiol Rev, 13(1), 144-166.

Vieusseux, M. (1805). Mémoire su la maladie qui a regné a Genêve au printemps de 1804. J Med Chir Pharmacol, 11, 163.

Vink, C., Rudenko, G., \& Seifert, H. S. (2012). Microbial antigenic variation mediated by homologous DNA recombination. FEMS Microbiol Rev, 36(5), 917-948.

Virji, M. (2009). Pathogenic neisseriae: surface modulation, pathogenesis and infection control. Nat Rev Microbiol, 7(4), 274-286.

Virji, M., Evans, D., Hadfield, A., Grunert, F., Teixeira, A. M., \& Watt, S. M. (1999). Critical determinants of host receptor targeting by Neisseria meningitidis and Neisseria gonorrhoeae: identification of Opa adhesiotopes on the N-domain of CD66 molecules. Mol Microbiol, 34(3), 538-551.

Virji, M., Makepeace, K., Ferguson, D. J. P., Achtman, M., \& Moxon, E. R. (1993). Meningococcal OPA and OPC proteins - their role in colonization and invasion of human epithelial and endothelial cells. Mol Microbiol, 10(3), 499-510.

Vogel, U., \& Frosch, M. (1999). Mechanisms of neisserial serum resistance. Mol Microbiol, 32(6), 1133-1139.

Vogelmann, R., \& Nelson, W. J. (2005). Fractionation of the epithelial apical junctional complex: reassessment of protein distributions in different substructures. $\mathrm{Mol}$ Biol Cell, 16(2), 701-716.

Wallace, D. F. (2016). The Regulation of Iron Absorption and Homeostasis. Clin Biochem Rev, $37(2), 51-62$.

Wang, S. A., Lee, M. V. C., O'Connor, N., Iverson, C. J., Ohye, R. G., Whiticar, P. M., Hale, J. A., Trees, D. L., Knapp, J. S., Effler, P. V., \& Weinstock, H. S. (2003). Multidrug-Resistant Neisseria gonorrhoeae with Decreased Susceptibility to Cefixime-Hawaii, 2001. Clin Infect Dis, 37(6), 849-852.

Wang, Y., Kinzie, E., Berger, F. G., Lim, S. K., \& Baumann, H. (2001). Haptoglobin, an inflammation-inducible plasma protein. Redox Rep, 6(6), 379-385.

Wassell, J. (2000). Haptoglobin: function and polymorphism. Clin Lab, 46(11-12), 547-552. 
Weichselbaum, A. (1887). Ueber die aetiologie der akuten meningitis cerebrospinalis. Fortschr. Med., 5, 573-583, 620-626.

Weinberg, E. D. (1975). Nutritional immunity. Host's attempt to withold iron from microbial invaders. Jama, 231(1), 39-41.

Weinberg, E. D. (2009). Iron availability and infection. Biochim Biophys Acta, 1790(7), 600-605.

Westendorp, R. G., Langermans, J. A., Huizinga, T. W., Elouali, A. H., Verweij, C. L., Boomsma, D. I., \& Vandenbroucke, J. P. (1997). Genetic influence on cytokine production and fatal meningococcal disease. Lancet, 349(9046), 170-173.

WHO. (2012a). Global incidence and prevalence of selected curable sexually transmitted infections-2008: World Health Organization.

WHO. (2012b). International Travel and Health. Geneva, Switzerland World Health Organisation.

Wickham, H. (2009). ggplot2: Elegant Graphics for Data Analysis: Springer Publishing Company, Incorporated.

Wilson, J. W., Schurr, M. J., LeBlanc, C. L., Ramamurthy, R., Buchanan, K. L., \& Nickerson, C. A. (2002). Mechanisms of bacterial pathogenicity. Postgrad Med J, 78(918), 216-224.

Wong, C. T., Xu, Y., Gupta, A., Garnett, J. A., Matthews, S. J., \& Hare, S. A. (2015). Structural analysis of haemoglobin binding by HpuA from the Neisseriaceae family. Nat Commun, 6, 10172.

Wooldridge, K. G., \& Williams, P. H. (1993). Iron uptake mechanisms of pathogenic bacteria. FEMS Microbiol Rev, 12(4), 325-348.

Yang, F., Friedrichs, W. E., Navarijo-Ashbaugh, A. L., deGraffenried, L. A., Bowman, B. H., \& Coalson, J. J. (1995). Cell type-specific and inflammatory-induced expression of haptoglobin gene in lung. Lab Invest, 73(3), 433-440.

Yao, M., Fang, W., Smart, C., Hu, Q., Huang, S., Alvarez, N., Fields, P., \& Cheng, N. (2018). CCR2 chemokine receptors enhance growth and cell cycle progression of breast cancer cells through SRC and PKC activation. Mol Cancer Res.

Yazdankhah, S. P., \& Caugant, D. A. (2004). Neisseria meningitidis: an overview of the carriage state. J Med Microbiol, 53(9), 821-832.

Zahm, J. M., Chevillard, M., \& Puchelle, E. (1991). Wound repair of human surface respiratory epithelium. Am J Respir Cell Mol Biol, 5(3), 242-248. 
Zahm, J. M., Kaplan, H., Herard, A. L., Doriot, F., Pierrot, D., Somelette, P., \& Puchelle, E. (1997). Cell migration and proliferation during the in vitro wound repair of the respiratory epithelium. Cell Motil Cytoskeleton, 37(1), 33-43.

Zhang, Y. J., \& Rubin, E. J. (2013). Feast or famine: the host-pathogen battle over amino acids. Cell Microbiol, 15(7), 1079-1087. 


\section{Appendix}

\subsection{Evans blue Transwell permeability}

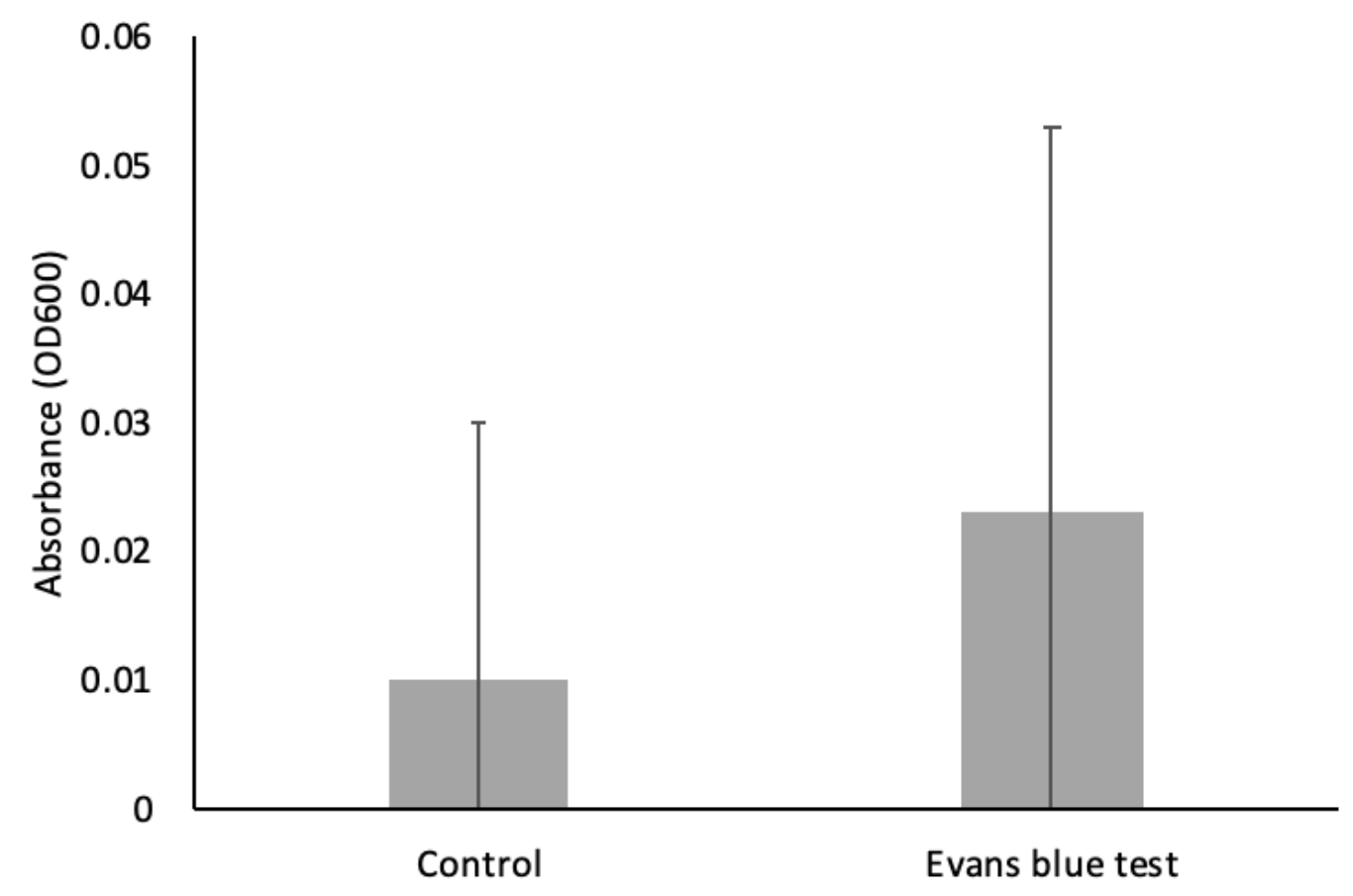

Figure 9.1. Assay testing leakage between apical layer and basal layer in Transwell system. This assay was carried out to check for tight junction formation in polarised cells. Evans blue should not be able to cross a barrier of epithelial cells forming tight junctions. Evans blue was added to the apical (upper) chamber and the absorbance of the medium in the lower chamber measured after 10 minutes. Control is M199 medium from basal layer, with no Evans blue added. Evans blue test was $500 \mu \mathrm{L}$ of Evans blue $(0.67 \mathrm{mg} / \mathrm{ml}$ ) (Sigma-Aldrich, Missouri, USA), diluted in M199 medium with 10\% FBS, added the apical chamber. No significant difference was found. $(n=4)$. 


\subsection{Expression levels of differentially regulated genes}

Table 9.1 RNA-seq expression levels of 48 genes most differentially expressed between NZ97/052 and NZCM112

\begin{tabular}{|c|c|c|c|c|c|}
\hline Gene name & NZ97/052-1 & NZ97/052-2 & NZCM112-1 & NZCM112-2 & Accession \\
\hline NMBG2136_1389 & 1627 & 662 & 306 & 167 & ADY94086 \\
\hline HрuB & 3612 & 1421 & 792 & 559 & ADY94518 \\
\hline NMBG2136_0974 & 792 & 195 & 229 & 124 & ADY93689 \\
\hline NMBG2136_0973 & 531 & 347 & 220 & 142 & ADY93688 \\
\hline $\mathrm{ZmpB}$ & 1307 & 450 & 494 & 338 & ADY94637 \\
\hline TсуA & 7961 & 2527 & 3308 & 2293 & ADY93462 \\
\hline GapA & 1931 & 656 & 811 & 527 & ADY92948 \\
\hline CysG & 407 & 179 & 141 & 113 & ADY93793 \\
\hline Opa & 26693 & 10516 & 13317 & 7599 & ADY94055 \\
\hline FumA & 7457 & 1925 & 2805 & 2066 & ADY94179 \\
\hline HрuA & 13260 & 17237 & 2563 & 9720 & ADY94157 \\
\hline Sbp & 927 & 347 & 368 & 279 & ADY93710 \\
\hline CysJ & 716 & 298 & 350 & 166 & ADY93789 \\
\hline NusB & 1556 & 607 & 773 & 508 & ADY93359 \\
\hline HsIR & 1811 & 369 & 2279 & 1557 & ADY93776 \\
\hline CytX & 2847 & 770 & 4746 & 2338 & ADY94614 \\
\hline NMBG2136_1356 & 1285 & 537 & 1761 & 2322 & ADY94053 \\
\hline FbpA & 18602 & 7907 & 32448 & 26320 & ADY93303 \\
\hline Bfd & 2430 & 710 & 4047 & 2751 & ADY93430 \\
\hline OmpU & 2506 & 922 & 4183 & 3515 & ADY94451 \\
\hline Mboll & 3932 & 955 & 6647 & 3773 & ADY93975 \\
\hline ExbB & 6128 & 2229 & 11016 & 8806 & ADY94276 \\
\hline $\operatorname{Nad} A$ & 1763 & 494 & 3025 & 2178 & ADY94540 \\
\hline TonB & 5955 & 1828 & 10981 & 7702 & ADY94277 \\
\hline $\mathrm{Nqr}$ & 28928 & 7837 & 52068 & 34421 & ADY93157 \\
\hline ExbD & 5440 & 1855 & 10319 & 8369 & ADY94275 \\
\hline NMBG2136_1142 & 4951 & 1264 & 9156 & 6295 & ADY93850 \\
\hline NMBG2136_1347 & 13054 & 3400 & 23053 & 18451 & ADY94044 \\
\hline FetB & 1133 & 401 & 2944 & 1929 & ADY94535 \\
\hline NMBG2136_1170 & 526 & 163 & 1598 & 1030 & NMBG2136_1170 \\
\hline NMBG2136_1275 & 2826 & 884 & 7198 & 5199 & ADY93976 \\
\hline NMBG2136_1168 & 336 & 136 & 1115 & 966 & NMBG2136_1168 \\
\hline NMBG2136_1543 & 2034 & 727 & 5604 & 4256 & NMBG2136_1543 \\
\hline EfeB & 1730 & 336 & 4436 & 2714 & ADY92798 \\
\hline TbpA & 5147 & 1752 & 13885 & 10327 & ADY94313 \\
\hline NMBG2136_0113 & 477 & 108 & 1368 & 1244 & ADY92864 \\
\hline EfeU & 754 & 374 & 3513 & 2598 & ADY92796 \\
\hline
\end{tabular}




\begin{tabular}{llllll}
\hline Gene name & NZ97/052-1 & NZ97/052-2 & NZCM112-1 & NZCM112-2 & Accession \\
\hline EfeO & & & & & \\
FumC & 4122 & 917 & 12346 & 9031 & ADY92797 \\
NMBG2136_1807 & 781 & 461 & 5320 & 3549 & ADY94048 \\
NMBG2136_1167 & 1361 & 260 & 3185 & 1994 & NMBG2136_1807 \\
TbpB & 4328 & 1562 & 17038 & 11551 & ADY94314 \\
ZupT & 60 & 38 & 1090 & 591 & ADY92918 \\
LbpB & 3330 & 770 & 17507 & 12855 & ADY94117 \\
LbpA & 1464 & 510 & 11820 & 8275 & ADY94116 \\
RhIE & 3314 & 911 & 24142 & 16647 & ADY93977 \\
NimA & 2907 & 813 & 29110 & 23342 & ADY93422 \\
FetA & 927 & 249 & 29763 & 18824 & ADY94534 \\
\hline
\end{tabular}




\subsection{Genes not involved in inhibition of epithelial cell migration.}

Table 9.2 lists genes which have previously been deleted from disease-associated isolates of meningococci at ESR, prior to the start of this project. None of the genes deleted had an impact on inhibiting cell migration. Most genes deleted had differential expression in diseaseassociated and carriage-associated household isolates, and many were deleted from a different strain, NZ98/254, a serogroup B New Zealand epidemic strain type that inhibits wound repair.

Table 9.2 Genes previously deleted from disease-associated meningococcal isolates, which did not impact wound repair inhibition.

\begin{tabular}{|c|c|c|}
\hline Gene name & Locus tag & Predicted Function of Encoded Protein \\
\hline $\operatorname{nad} A$ & NMBG2136_1708 & $\begin{array}{l}\text { Promotes adhesion and invasion into } \\
\text { epithelial cells }\end{array}$ \\
\hline zupT & NMBG2136_0171 & Zinc transporter \\
\hline & NMBNZ0533_1525/ & \\
\hline$I b p A / I b p B$ & NMBNZ0533_1526 & Lactoferrin binding proteins \\
\hline pilCl & NMBNZ0533_0475 & Involved in pili assembly \\
\hline porA & NMBNZ0533_1408 & Outer membrane porin protein \\
\hline por $B$ & NMBNZ0533_1971 & Major outer membrane protein IB \\
\hline $\operatorname{sia} D$ & NMBNZ0533_0073 & Polysialic acid capsule biosynthesis protein \\
\hline pilA & $\begin{array}{l}\text { NMBNZ0533_0019- } \\
\text { NMBNZ0533_0031 }\end{array}$ & Pilin expression locus \\
\hline opcA & NMBNZ0533_1078 & $\begin{array}{l}\text { Outer membrane protein. Mediates } \\
\text { attachment to host cells by binding } \\
\text { proteoglycan cell surface receptors }\end{array}$ \\
\hline hap (App) & NMBNZ0533_0338 & Adhesion penetration protein \\
\hline
\end{tabular}




\begin{tabular}{lll} 
ompA & NMBNZ0533_1864 & $\begin{array}{l}\text { Outer membrane protein. Role in interaction } \\
\text { with host cells }\end{array}$ \\
gdhA & NMBNZ0533_1685 & Encodes glutamate dehydrogenase \\
IgtA & NMBNZ0533_0398 & $\begin{array}{l}\text { Lacto-N-neotetraose biosynthesis glycosyl } \\
\text { transferase }\end{array}$ \\
IgtC & NMBNZ0533_0387 & $\begin{array}{l}\text { Lacto-N-neotetraose biosynthesis glycosyl } \\
\text { transferase }\end{array}$ \\
NMBNZ0533_ & NMBNZ0533_0093 & $\begin{array}{l}\text { Conserved hypothetical protein with a } \\
\text { predicted outer membrane protein domain. } \\
\text { 0093 }\end{array}$ \\
prmB & NMBNZ0533_1628 & $\begin{array}{l}\text { 50S ribosomal protein L3 glutamine } \\
\text { methyltransferase }\end{array}$ \\
\hline
\end{tabular}




\title{
9.4 Sequences of gene deletion mutants
}

In-house Sanger sequencing was completed to sequence the PCR products of the gene regions of interest from the mutagenic isolates. Regions that were sequenced were the $h p u A$ gene region of NZ97/052 $\triangle$ hpuA mutant (Figure 9.2), the $h p u B$ region of the NZ97/052 $\Delta h p u B$ mutant (Figure 9.3), and $h p u A B$ gene region of the NZ97/052 $\triangle h p u A B$ mutant (Figure 9.4).

\begin{abstract}
CAAAGCCCTGTCCTTACTGCCGCTTGTCGCTGCCCTTGCCGCCTGTGCGGGGGGGGGGGGG TAGCCGAACCGCACGTCCCCGTGTCCATCCCCACCGCCACGCCGCTGACCGGTGAGGTAAAG TTAACAGACGACAATAGCAAAATCGAAAACATCAACACCGCCAACACTGGAAACACATCCGG CGGAATTCGCCGTCTGAACCAGCGAACCATTTGAGGTGATAGGTAAGATTATACCGAGGAAT GAAAACGAGAATTGGACCTTTACAGAATTACTCTATGAAGCGCCATATTTAAAAAGCTACCA AGACGATAAATGCGTCGGAAAAGTTAAACTGCGAAAAAATTGGAACCGGTACGCTTATATAG AAGATATCGCCGTATGTAAGGATTTCACGGGGCAAGGCATAGGCAGCGCGCTTATCAATATA TCCATAGAATGGGCAAAGCATAAAAACTTGCATGGACTAATGCTTGAAACCCAGACAATAAC CTTATAGCTTGTAAATTCTATCATAATTGTGGTTTCAAAATCGGCTCCGTCGATACTATGTT ATACGCCAACTTTGAAAACAACTTTGAAAAGCTGTTTCTGGTGTTTAGTTTTAGATGCAAGA ACAGTGAATTGAGTTCGTCTGTATATAGCTTCTTGGGGTATCTTTAAATACTGTAGAAAGAG ATAAATAATAAATGCTAGATGAATATCACCGATTGAAAAACTGATCGAAAAATACGCTGCGT AAAGATACGGACGATGTCTCTCTGCTAGTATATAATCTGGTGGAAATGGAACTATTTTAAAT GACGAACGCCCGTACGACCCTAGAGTTGGCACCGAGGAAGCGAGTATTACGCTGTGGCCAAT GAACCTAATGATGCGGGACAGCGTTAAGTACACCTGATTGGACGAAAGACCTGTGCTATGCT GAGAAGCTGCGTTCACGTCTGCACTTGACGCATGATGCTGAGCATCTGTCATGAGTGAGGCC GATGCGTCTTGCTGAGAGTATGAGATGACCAAAGCTGAAAGATATCGAGCTGTATGCGAGTG CATCAGCTCTTCACTCCATCGACATATCGGATTGTCCCTATACGAATAGCTTAGACAGCCGC TAGCCGAATTGGATTACTTACTGAATAACGATCTGGCCGATGTGGATTGCGAAAACTGGGAA GAAGACACTCCATTTAAAGATCCGCGCGAGCTGTATGATTTTTAAAGACGGAAAAGCCCGAA GAGGAACTTGTCTTTTCCCACGGCGACTGGGAGACAGCAACATCTTTGTGAAAGATGGCAAA GTAAGTGGCTTTATTGATCTTGGGAGAAGCGGCAGGGCGGACAAGTGGTATGACATTGCCTT CTGCGTCCGGTCGATCAGGGAGGATATCGGGGAAGACAGTATGTCGAGCTATTTTTTGACT TACTGGGGATCAAGCCTGATTGGGAGAAAATAAAATATTATATTTTACTGGATGAATTGTTT TAGTACCTAGATTTAGATGTCTAAAAAGCGAATTCCGCAGATGTCTAAAAAGCGAATTCCGC TATCACCGCCAATTTCAACACCCATAAACTGTCAGGCGAAATCATCGGCAACAGCGATTACG GGCCAAATGTGAAAATTGAAAACGCAACGATTGACGGACTATCCTTTTCCGGCGATGCCACG TCCGGCGGCAAAAACGGCAAATTGGAAG
\end{abstract}

Figure 9.2. Sequence of $h p u A$ region from NZ97/052 $\triangle h p u A$ gene deletion mutant. Primer sequences are underlined. Bold indicates aphA3 sequence. Sequence corresponding to N. meningitidis NZ97/052 genome sequence is not in bold. The F1 and R4 primers both annealed fully inside the coding sequence of the target gene. $814 \mathrm{bp}$ of the total predicted coding region, $1029 \mathrm{bp}$, was removed. 


\begin{abstract}
ATCCCCTTCAAACCCGTATTGGCTGCCGCCGCCATCGCCCAAGCGTTTCCCGCCTTTGCGGC AGACCCCGCGCCGCAGTCCGCCCAAACGCTGAACGAAATCACCGTTACCGGCACGCACAAAA CCCAAAAACTCGGCGAAGAAAAAATCCGCCGCAAAACTTTAGACAAGCTCTTGGTCAACGAC GAACAGCGGAATTCGCCGTCTGAACCAGCGAACCATTTGAGGTGATAGGTAAGATTATACCG AGGAATGAAAACGAGAATTGGACCTTTACAGAATTACTCTATGAAGCGCCATATTTAAAAAG CTACCAAGACGATAAATGCGTCGGAAAAGTTAAACTGCGAAAAAATTGGAACCGGTACGCTT ATATAGAAGATATCGCCGTATGTAAGGATTTCACGGGGCAAGGCATAGGCAGCGCGCTTATC AATATATCCATAGAATGGGCAAAGCATAAAAACTTGCATGGACTAATGCTTGAAACCCAGAC AATAACCTTATAGCTTGTAAATTCTATCATAATTGTGGTTTCAAAATCGGCTCCGTCGATAC TATGTTATACGCCAACTTTGAAAACAACTTTGAAAAGCTGTTTCTGGTGTTTAGTTTTAGAT GCAAGAACAGTGAATTGAGTTCGTCTGTATATAGCTTCTTGGGGTATCTTTAAATACTGTAG AAAGAGATAAATAATAAATGCTAGATGAATATCACCGATTGAAAAACTGATCGAAAAATACG CTGCGTAAAGATACGGACGATGTCTCTCTGCTAGTATATAATCTGGTGGAAATGGAATATT TTAAATGACGAACGCCCGTACGACCCTAGAGTTGGCACCGAGGAAGCGAGTATTACGCTGTG GCCAATGAACCTAATGATGCGGGACAGCGTTAAGTACACCTGATTGGACGAAAGACCTGTGC TATGCTGAGAAGCTGCGTTCACGTCTGCACTTGACGCATGATGCTGAGCATCTGTCATGAGT GAGGCCGATGCGTCTTGCTGAGAGTATGAGATGACCAAAGCTGAAAGATATCGAGCTGTATG CGAGTGCATCAGCTCTTCACTCCATCGACATATCGGATTGTCCCTATACGAATAGCTTAGAC AGCCGCTAGCCGAATTGGATTACTTACTGAATAACGATCTGGCCGATGTGGATTGCGAAAAC TGGGAAGAAGACACTCCATTTAAAGATCCGCGCGAGCTGTATGATTTTTAAAGACGGAAAAG CCCGAAGAGGAACTTGTCTTTTCCCACGGCGACTGGGAGACAGCAACATCTTTGTGAAAGAT GGCAAAGTAAGTGGCTTTATTGATCTTGGGAGAAGCGGCAGGGCGGACAAGTGGTATGACAT TGCCTTCTGCGTCCGGTCGATCAGGGAGGATATCGGGGAAGAACAGTATGTCGAGCTATTTT TTGACTTACTGGGGATCAAGCCTGATTGGGAGAAAATAAAATATTATATTTTACTGGATGAA TTGTTTTAGTACCTAGATTTAGATGTCTAAAAAGCGAATTCCGCAGATGTCTAAAAAGCGAA TTCCGCAAAACAACGGCAAAGAAACGCCCATCAACGCGCTTTCGCCGTGGACGGCGGTTTAC AGCCTGGGCTATGACGCGCCTTCCAAACGCTGGGGCATCAACGCCTACGCCACGCGCACCGC CGCCAAAAAGCCGTCCGACACCGTCCACAGCAACGACGACTTGAACAACCCGTGGCCTTATG CCAAACACAGCAAGGCCTATACGCTGTTCGACCTTTCCGCСTACCTCAACATC
\end{abstract}

Figure 9.3. Sequence of $h p u B$ region from NZ97/052 $\triangle h p u B$ gene deletion mutant. Primer sequences are underlined. Bold indicates aphA3 sequence. Sequence corresponding to N. meningitidis NZ97/052 genome sequence is not in bold. The F1 and R4 primers both annealed fully inside the coding sequence of the target gene. $1985 \mathrm{bp}$ of the total predicted coding region, $2433 \mathrm{bp}$, was removed. 
CAAAGCCCTGTCCTTACTGCCGCTTGTCGCTGCCCTTGCCGCCTGTGCGGGGGGGGGGGGGG TAGCCGAACCGCACGTCCCCGTGTCCATCCCCACCGCCACGCCGCTGACCGGTGAGGTAAAG TTAACAGACGACAATAGCAAAATCGAAAACATCAACACCGCCAACACTGGAAACACATCCGG CGGAATTCGCCGTCTGAACCAGCGAACCATTTGAGGTGATAGGTAAGATTATACCGAGGAAT GAAAACGAGAATTGGACCTTTACAGAATTACTCTATGAAGCGCCATATTTAAAAAGCTACCA AGACGATAAATGCGTCGGAAAAGTTAAACTGCGAAAAAATTGGAACCGGTACGCTTATATAG AAGATATCGCCGTATGTAAGGATTTCACGGGGCAAGGCATAGGCAGCGCGCTTATCAATATA TCCATAGAATGGGCAAAGCATAAAAACTTGCATGGACTAATGCTTGAAACCCAGACAATAAC CTTATAGCTTGTAAATTCTATCATAATTGTGGTTTCAAAATCGGCTCCGTCGATACTATGTT ATACGCCAACTTTGAAAACAACTTTGAAAAGCTGTTTCTGGTGTTTAGTTTTAGATGCAAGA ACAGTGAATTGAGTTCGTCTGTATATAGCTTCTTGGGGTATCTTTAAATACTGTAGAAAGAG ATAAATAATAAATGCTAGATGAATATCACCGATTGAAAAACTGATCGAAAAATACGCTGCGT AAAGATACGGACGATGTCTCTCTGCTAGTATATAATCTGGTGGAAATGGAACTATTTTAAAT GACGAACGCCCGTACGACCCTAGAGTTGGCACCGAGGAAGCGAGTATTACGCTGTGGCCAAT GAACCTAATGATGCGGGACAGCGTTAAGTACACCTGATTGGACGAAAGACCTGTGCTATGCT GAGAAGCTGCGTTCACGTCTGCACTTGACGCATGATGCTGAGCATCTGTCATGAGTGAGGCC GATGCGTCTTGCTGAGAGTATGAGATGACCAAAGCTGAAAGATATCGAGCTGTATGCGAGTG CATCAGCTCTTCACTCCATCGACATATCGGATTGTCCCTATACGAATAGCTTAGACAGCCGC TAGCCGAATTGGATTACTTACTGAATAACGATCTGGCCGATGTGGATTGCGAAAACTGGGAA GAAGACACTCCATTTAAAGATCCGCGCGAGCTGTATGATTTTTAAAGACGGAAAAGCCCGAA GAGGAACTTGTCTTTTCCCACGGCGACTGGGAGACAGCAACATCTTTGTGAAAGATGGCAAA GTAAGTGGCTTTATTGATCTTGGGAGAAGCGGCAGGGCGACAAGTGGTATGACATTGCCTT CTGCGTCCGGTCGATCAGGGAGGATATCGGGGAAGAACAGTATGTCGAGCTATTTTTTGACT TACTGGGGATCAAGCCTGATTGGGAGAAAATAAAATATTATATTTTACTGGATGAATTGTTT TAGTACCTAGATTTAGATGTCTAAAAAGCGAATTCCGCAGATGTCTAAAAAGCGAATTCCGC AAAACAACGGCAAAGAAACGCCCATCAACGCGCTTTCGCCGTGGACGGCGGTTTACAGCCTG GGCTATGACGCGCCTTCCAAACGCTGGGGCATCAACGCCTACGCCACGCGCACCGCCGCCAA AAAGCCGTCCGACACCGTCCACAGCAACGACGACTTGAACAACCCGTGGCCTTATGCCAAAC ACAGCAAGGCCTATACGCTGTTCGACCTTTCCGCCTACCTCAACATC

Figure 9.4. Sequence of $h p u A B$ region from NZ97/052 $\triangle h p u A B$ gene deletion mutant. Primer sequences are underlined. Bold indicates aphA3 sequence. Sequence corresponding to N. meningitidis NZ97/052 genome sequence is not in bold. The F1 and R4 primers both annealed fully inside the coding sequences of the target genes. $3061 \mathrm{bp}$ of the total predicted coding region, $3492 \mathrm{bp}$, was removed. 


\subsection{Sulfur uptake pathway in $N$. meningitidis}

While carrying out work for my main project, during my second year I was also able to assist with completing experiments for another paper, as requested by reviewers. Experiments completed were glutathione assays, hydrogen peroxide sensitivity assays, and quantitative realtime PCR in the NZ97/192 household. Glutathione assays and hydrogen peroxide sensitivity assays are incorporated into Figure 5A and Figure 5B of the paper, 'Genomic, Transcriptomic and Phenotypic Analysis of Neisseria meningitidis Isolates from Disease Patients and Their Household Contacts', which is appended below (Appendix I).

\subsubsection{Glutathione Assay protocol}

To measure intracellular glutathione, $N$. meningitidis was scraped from fresh plates resuspended in PBS to an $\mathrm{OD}_{600}$ of 1.0. Cells were centrifuged at 4,000 rpm for 10 minutes. Three volumes of $5 \%$ sulfosalicyclic acid was added to the cells. Samples were freeze-thawed twice in liquid nitrogen then left for five minutes at $4^{\circ} \mathrm{C}$. Samples were then centrifuged at 13,000 rpm for 10 minutes. Glutathione concentrations in the supernatants were measured with glutathione assay kit (Sigma-Aldrich, Missouri, USA) according to manufacturer's instructions. Experiments were performed in triplicate, three independent times.

\subsubsection{Hydrogen peroxide sensitivity assay protocol}

N. meningitidis was scraped from fresh plates and resuspended in $\mathrm{BHI}$ broth. Cultures were incubated at $37^{\circ} \mathrm{C}$ with $5 \% \mathrm{CO}_{2}$ until $\mathrm{OD}_{600}$ reached 0.6 . Cultures were split into three aliquots of $1 \mathrm{~mL}$ with increasing concentrations $(0,2$ and $5 \mathrm{mM})$ of stabilized hydrogen peroxide $\left(\mathrm{H}_{2} \mathrm{O}_{2}\right)$ (Sigma-Aldrich, Missouri, USA). The cultures were incubated at $37^{\circ} \mathrm{C}$ with $5 \% \mathrm{CO}_{2}$ for 20 minutes. After this incubation step, to remove excess $\mathrm{H}_{2} \mathrm{O}_{2}$, bacteria were harvested by centrifugation at $2000 \mathrm{rpm}$. Supernatant was discarded and pellets were resuspended in $300 \mu \mathrm{L}$ BHI. Cells were then collected by centrifugation at 13,000 rpm, supernatant was discarded, and pellets were resuspended in $1 \mathrm{~mL}$ BHI. Viable bacteria were evaluated by CFU on CBA plates (Fort Ricard Laboratories). The experiments were performed in triplicate, two independent times. 


\subsubsection{Quantitative real-time PCR}

Quantitative real-time PCR (qRT-PCR) was completed to measure expression levels of $c y s G$ and NZBNZ0533_0037 expression in the NZ97/192 household isolates. Realtime primers and probes were designed with the RealTimeDesign software (BioSearch Technologies, Inc) and were generated by Life Technologies (Table 9.3). The specificity of PCR primers was determined by PCR followed by analysis with agarose gel electrophoresis. PCR assays were carried out using TaqMan Universal Master Mix II with uracil-N glycosylase (UNG) (Thermo Fisher Scientific, USA), according to the manufacturer's instruction. For ease, $250 \mathrm{nM}$ probe and $200 \mathrm{nM}$ primers were used for all reactions. Cycle parameters are detailed in Table 9.4. Reaction efficiency was determined using a standard curve with increasing concentrations of genomic DNA. The meningococcal genes aroE and ctrA were used as controls, as they did not differ significantly between isolates according to RNAseq data. Measurements were performed in parallel, and experiment was performed three individual times from separate cell cultures. Statistical significance was determined using unpaired t-test.

Table 9.3. Primers and probes used for qRT-PCR

\begin{tabular}{|c|c|c|c|}
\hline Target & $\begin{array}{l}\text { Primer/Probe } \\
\text { name }\end{array}$ & Nucleotide sequence $5^{\prime}-3^{\prime}$ & $\begin{array}{l}\text { Fluorescent } \\
\text { label }\end{array}$ \\
\hline \multirow{3}{*}{ cysG } & CysG_F & GCGGACGAGGGACAGTATG & \\
\hline & CysG_R & GGCTGCTGACGCTCAAAG & \\
\hline & CysG_P & CCGTCTGAAACCAGCGCGTCGT & FAM-BHQ1 \\
\hline \multirow{3}{*}{ NMBNZ0533_0037 } & 0037_F & ACGACGGGAAAGAGCTGTATG & \\
\hline & 0037_R & TCATCGCCGCATCGGTTT & \\
\hline & 0037_P & TCGAACGCAGGCAGTATGTCA & FAM-BHQ1 \\
\hline \multirow{3}{*}{$\operatorname{ctr} A$} & CtrA_F & TTGTGTGGAAGTTTAATTGTAGGATGC & \\
\hline & CtrA_R & TCAGATTGTTGCCCTAAAGAGACA & \\
\hline & CtrA_P & TCCTTCATCAGGCCCCAGCG & FAM-BHQ1 \\
\hline \multirow[t]{3}{*}{ aroE } & AroE_F & GTCCTGCCCGTATCGTCAT & \\
\hline & AroE_R & TCGGGACGGCTTCAATGC & \\
\hline & AroE_P & CCAACCGTACCCATGCCAAAGCC & FAM-BHQ1 \\
\hline
\end{tabular}


Table 9.4. Real time PCR cycle parameters.

\begin{tabular}{lll}
\hline Number of cycles & Temperature & Duration \\
\hline 1 cycle & $52^{\circ} \mathrm{C}$ & 60 seconds \\
1 cycle & $95^{\circ} \mathrm{C}$ & 10 minutes \\
45 cycles & $95^{\circ} \mathrm{C}$ & 10 seconds \\
& $58^{\circ} \mathrm{C}$ & 60 seconds \\
\hline
\end{tabular}

Transcriptomic analysis suggested these genes were upregulated in the disease-associated isolate. There was high variability between qRT-PCR replicates, however available data suggests that transcriptome data was correct in identifying CysG as down regulated in carriage associated isolates, relative to NZ97/192 (Figure 9.5). NMBNZ0533_0037 is predicted to encode a phase variable protein so data sets were widely varied. Results from experiments examining virulence in NZ97/192 house hold isolates suggest the sulfute assimilation pathway may be a potentially important metabolic difference between carriage-associated and disease associated isolates. 


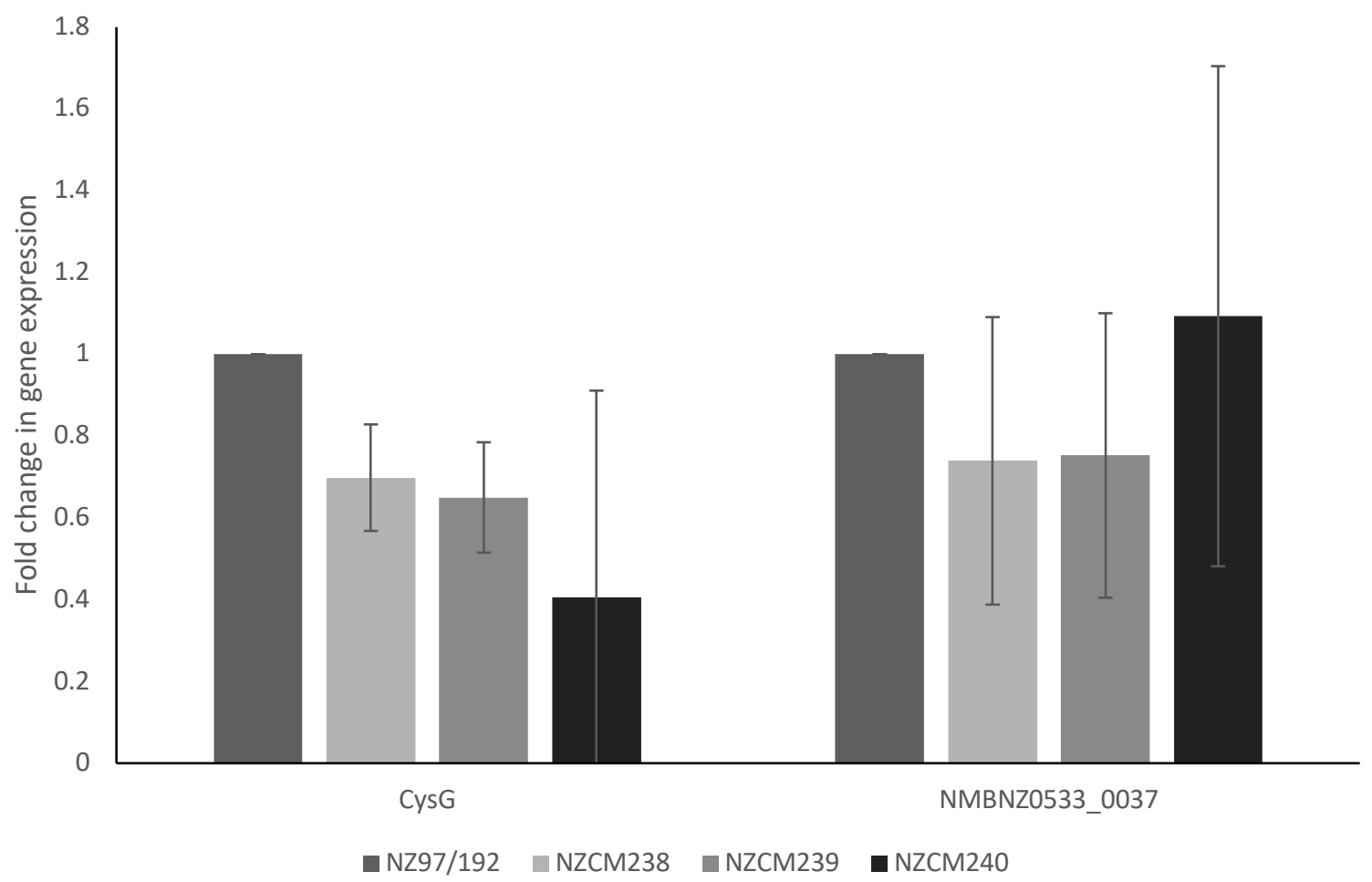

Figure 9.5. Gene expression of CysG and NMBNZ0533_0037 in NZ97/192 household isolates. Error bars represent standard deviations. Experiment was performed three times with eight technical replicates. 


\subsection{Nucleotide and predicted protein sequences of hpuA}

Figure 9.6 shows the nucleotide sequence and associated predicted protein sequence of $h p u A$ in NZ97/052. Figure 9.7 is the codon optimized $h p u A$ sequence and predicted protein sequence. Predicted protein sequences of NZ97/052 hpuA and the codon optimised $h p u A$ were aligned using ClustalW (Thompson et al., 1994), and demonstrate the predicted proteins sequences are identical, barring the but with a His-tag and other amino acids at the $\mathrm{N}$-terminus (Figure 9.8). 
atgaaatacaaagccetgtccttactgccgcttgtcgctgccettgcogcctgtgcgggg $\begin{array}{lllllllllllllllllllllllll}M & K & Y & K & A & L & S & L & L & P & L & V & A & A & L & A & A & C & A & G\end{array}$ ggggggggggtagccgaaccgcacgtcccegtgtccatccccaccgccacgccgetgacc $\begin{array}{llllllllllllllllllll}G & G & G & V & A & E & P & H & V & P & V & S & I & P & T & A & T & P & L & T\end{array}$ ggtgaggtaaagttaacagacgacaatagcaaaatcgaaaacatcaacaccgccaacact $\begin{array}{llllllllllllllllllll}G & E & V & K & L & T & D & D & N & S & K & I & E & N & I & N & T & A & N & T\end{array}$ ggaaacacatccggcatttccattcagcagcgggaatataaagtaaacaattacggcgtc $\begin{array}{llllllllllllllllllll}G & N & T & S & G & I & S & I & Q & Q & R & E & Y & K & V & N & N & Y & G & V\end{array}$ gaatcaacagcaaaagcattcatcttcaaaacacctggcggcgcgcaatacaccctttca $\begin{array}{llllllllllllllllllll}\mathbf{E} & \mathrm{S} & \mathrm{T} & \mathrm{A} & \mathrm{K} & \mathrm{A} & \mathrm{F} & \mathrm{I} & \mathrm{F} & \mathrm{K} & \mathrm{T} & \mathrm{P} & \mathrm{G} & \mathrm{G} & \mathrm{A} & \mathrm{Q} & \mathrm{Y} & \mathrm{T} & \mathrm{L} & \mathrm{S}\end{array}$ tcctacgccgaccccatcgtccccagctactcctccccogatttcaaattcccgaccgc $\begin{array}{llllllllllllllllllll}S & Y & A & D & P & I & V & P & S & Y & S & S & P & D & F & K & I & P & D & R\end{array}$ cacgcagggcagcggcttgccgacggcagccgcatctttatctgctgcagcgactccggg

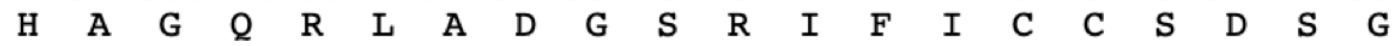
gcaaccaatcaagcggaaattaccaaacaagattatatgaaattcggggcgtggataggt $\begin{array}{llllllllllllllllllll}A & T & N & Q & A & E & I & T & K & Q & D & Y & M & K & F & G & A & W & I & G\end{array}$ cccaacggcgaaatcgacctcttcgccggcggcttccccgtcggcaaaacaccggcatca $\begin{array}{llllllllllllllllllll}P & N & G & E & I & D & L & F & A & G & G & F & P & V & G & K & T & P & A & S\end{array}$ agcagttattacggcagcagcacgcccgaaacccaaggcaagggcaaaatcacttatcag $\begin{array}{llllllllllllllllllll}S & S & Y & Y & G & S & S & T & P & E & T & Q & G & K & G & K & I & T & Y & Q\end{array}$ gtttggggcatccgcgtcagaaacgggcaatttgttacttcctcttatacgccgccgaaa

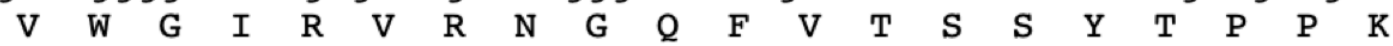
aacagtggttattattcgtccacacctaccaacacccccgtcctttcctttatcaccgcc $\begin{array}{llllllllllllllllllll}N & S & G & Y & Y & S & S & T & P & T & N & T & P & V & L & S & F & I & T & A\end{array}$ aatttcaacacccataaactgtcaggcgaaatcatcggcaacagcgattacgggccaat $\begin{array}{llllllllllllllllllll}\mathrm{N} & \mathrm{F} & \mathrm{N} & \mathrm{T} & \mathrm{H} & \mathrm{K} & \mathrm{L} & \mathrm{S} & \mathrm{G} & \mathrm{E} & \mathrm{I} & \mathrm{I} & \mathrm{G} & \mathrm{N} & \mathrm{S} & \mathrm{D} & \mathrm{Y} & \mathrm{G} & \mathrm{P} & \mathrm{N}\end{array}$ gtgaaattgaaaacgcaacgattgacggactatccttttccggcgatgccacgtccggc $\begin{array}{llllllllllllllllllll}V & K & I & E & N & A & T & I & D & G & L & S & F & S & G & D & A & T & S & G\end{array}$ ggcaaaacggcaaattggaaggcaagttttttggaaattcaacggctaccgggacact $\begin{array}{llllllllllllllllllll}G & K & N & G & K & \text { L } & E & G & K & F & F & G & K & F & N & G & Y & R & D & T\end{array}$ gaaaccagcataggcggcaaatcacctttgacggcgaccgctccctcgataccgtattc

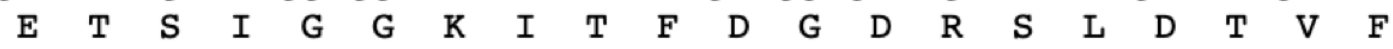
ggcggtgtgagttataaaaagaacttgaaaacaacactgatacaagtaccacccatctc $\begin{array}{llllllllllllllllllll}G & G & V & S & Y & K & K & E & L & E & N & N & T & D & T & S & T & T & H & L\end{array}$ acaaaataa

T K -

Figure 9.6. Nucleotide and predicted protein sequences of $h p u A$ in NZ97/052. Lower case is the nucleic acid sequence. Upper case is the associated predicted protein sequence. The start codon is in bold. Grey shading indicates the homopolymeric G-tract that is prone to slipped strand mispairing. 
catatgaaatataaagccetttcactgttgccgctggttgctgctcttgcggcttgtgcg $\begin{array}{llllllllllllllllllll}H & M & K & Y & K & A & L & S & L & L & P & L & V & A & A & L & A & A & C & A\end{array}$ ggtggtggtggcgtcgccgaaccgcacgtacccgtcagcatcccaaccgctacaccctta $\begin{array}{llllllllllllllllllll}G & G & G & G & V & A & E & P & H & V & P & V & S & I & P & T & A & T & P & L\end{array}$ accggggaggttaagttaactgacgacaatagcaaatcgaaatatcaacaccgccaat $\begin{array}{lllllllllllllllllllll}T & G & E & V & K & L & T & D & D & N & S & K & I & E & N & I & N & T & A & N\end{array}$ acaggaaatacgagtggtatctctattcaacagcgcgagtataaggtcaataattacggt

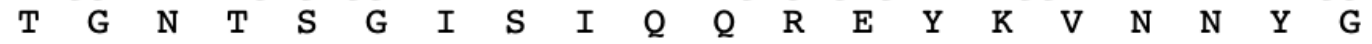
gtcgagtctacagctaaggcattcattttcaagactcctggcggtgcccagtatacactg $\begin{array}{llllllllllllllllllll}V & E & S & T & A & K & A & F & I & F & K & T & P & G & G & A & Q & Y & T & L\end{array}$ tcgagctatgctgaccccattgtccctagctattcaagtcccgattttaaaatcccagat $\begin{array}{llllllllllllllllllll}S & S & Y & A & D & P & I & V & P & S & Y & S & S & P & D & F & K & I & P & D\end{array}$ cgtcatgcaggacaacgccttgcagacggttcacgcatttttatttgttgctccgattcc $\begin{array}{llllllllllllllllllll}R & H & A & G & Q & R & L & A & D & G & S & R & I & F & I & C & C & S & D & S\end{array}$ ggcgcgactaatcaggccgagattaccaaacaggattacatgaaatttggtgcttggatt $\begin{array}{llllllllllllllllllll}G & A & T & N & Q & A & E & I & T & K & Q & D & Y & M & K & F & G & A & W & I\end{array}$ ggcccaaatggggagattgatttattcgcaggcggctttccagtaggaaaacaccggca $\begin{array}{llllllllllllllllllll}G & P & N & G & E & I & D & L & F & A & G & G & F & P & V & G & K & T & P & A\end{array}$ tcatcaagttactacggctcgtcaacacccgaaacgcagggcaagggtaaatcacctat $\begin{array}{llllllllllllllllllll}S & S & S & Y & Y & G & S & S & T & P & E & T & Q & G & K & G & K & I & T & Y\end{array}$ caggtctggggtattcgcgtccgcaatggacaattcgtcacctcttcttatacaccccct $\begin{array}{llllllllllllllllllll}Q & V & W & G & I & R & V & R & N & G & Q & F & V & T & S & S & Y & T & P & P\end{array}$ aagaattcagggtattacagctcgacacctaccaatactccggtcctttcttttatcact $\begin{array}{llllllllllllllllllll}K & N & S & G & Y & Y & S & S & T & P & T & N & T & P & V & L & S & F & I & T\end{array}$ gcaaacttcaacacccacaagctttcaggcgaatcatcggaaacagcgattacggccet $\begin{array}{llllllllllllllllllll}A & N & F & N & T & H & K & L & S & G & E & I & I & G & N & S & D & Y & G & P\end{array}$ aacgttaaaattgaaaacgctactattgacgggcttagcttttccggggatgcgacttcc $\begin{array}{llllllllllllllllllll}N & V & K & I & E & N & A & T & I & D & G & L & S & F & S & G & D & A & T & S\end{array}$ gggggcaagaatggcaagcttgaggggaagttcttcggcaaattcaacggatatcgtgat $\begin{array}{lllllllllllllllllllll} & G & G & K & N & G & K & L & E & G & K & F & F & G & K & F & N & G & Y & R & D\end{array}$ actgaaacgagtattggtggtaagatcacgttcgatggtgatcgttccttggatacggta $\begin{array}{llllllllllllllllllll}T & E & T & S & I & G & G & K & I & T & F & D & G & D & R & S & L & D & T & V\end{array}$ tttggcggagtatcatataagaaggagcttgaaaacaacacggatacttctacgacacac

$\begin{array}{lllllllllllllllllllll}\mathbf{F} & G & G & V & S & Y & K & K & E & \text { L } & E & N & N & T & D & T & S & T & T & H\end{array}$ ctgacgaaataactcgag

L $T$ K - L E

Figure 9.7. Codon optimised nucleotide sequence and predicted protein sequence of $h p u A$. Lower case is the nucleic acid sequence. Upper case is the associated predicted protein sequence. In bold is the start codon.Underlined sequences represent restriction sites. First restriction site; Ndel, Second restriction site; Xhol. 
$97 / 052$

Codon_optimised

$97 / 052$

Codon_optimised

$97 / 052$

Codon_optimised

120

121

1 -MKYKALSLLPLVAALAACAGGGGVAEPHVPVSI PTATPLTGEVKLTDDNSK IEN INTAN

1 HMKYKALSLLPLVAALAACAGGGGVAEPHVPVSI PTATPLTGEVKLTDDNSKIENINTAN

$97 / 052$

60 TGNTSGI I $Q Q R E Y K V N N Y$ GVESTAKAF IFKT PGGA $Q Y T L S S Y A D P I V P S Y S S P D F K$ I PD

Codon_optimised

180

181

$97 / 052$

240

Codon_optimised

ANF NTHKLSGE I I GNSDY GPNVK I ENAT I DGLSF SGDAT SGGKNGKLEGKFF GK FNGYRD

$97 / 052$

300

Codon_optimised

301

Figure 9.8. Protein sequence alignment of HpuA sequence in NZ97/052 and codon optimised HpuA sequence.

Aligned using ClustalW (Thompson et al., 1994). Images created using BOXSHADE 3.21. Black shading indicates conserved residues. His-tag at position one is indicated by dark grey shading. The linker sequence at the $\mathrm{N}$ terminus is indicated by light grey shading. His-tag and linker sequence are not expected to alter the protein function. 


\section{Genomic, Transcriptomic, and Phenotypic Analyses of Neisseria meningitidis Isolates from Disease Patients and Their Household Contacts}

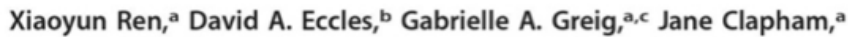 \\ Nicole E. Wheeler, d,e Stinus Lindgreen, d,f Paul P. Gardner, d \\ (D) Joanna K. MacKichanc,g \\ Invasive Pathogens Laboratory, Institute of Environmental Science and Research, Porirua, New Zealanda; \\ Malaghan Institute of Medical Research, Wellington, New Zealand ${ }^{\mathrm{b}}$; School of Biological Sciences, Victoria \\ University of Wellington, Wellington, New Zealand; ; School of Biological Sciences, University of Canterbury, \\ Christchurch, New Zealandd; Wellcome Trust Sanger Institute, Hinxton, United Kingdome; H. Lundbeck AVS, \\ Valby, Denmark; Centre for Biodiscovery, Victoria University of Wellington, Wellington, New Zealand 9
}

\begin{abstract}
Neisseria meningitidis (meningococcus) can cause meningococcal disease, a rapidly progressing and often fatal disease that can occur in previously healthy children. Meningococci are found in healthy carriers, where they reside in the nasopharynx as commensals. While carriage is relatively common, invasive disease, associated with hypervirulent strains, is a comparatively rare event. The basis of increased virulence in some strains is not well understood. New Zealand suffered a protracted meningococcal disease epidemic, from 1991 to 2008. During this time, a household carriage study was carried out in Auckland: household contacts of index meningococcal disease patients were swabbed for isolation of carriage strains. In many households, healthy carriers harbored strains identical, as determined by laboratory typing, to the ones infecting the associated patient. We carried out moredetailed analyses of carriage and disease isolates from a select number of households. We found that isolates, although indistinguishable by laboratory typing methods and likely closely related, had many differences. We identified multiple genome variants and transcriptional differences between isolates. These studies enabled the identification of two new phase-variable genes. We also found that several carriage strains had lost their type IV pili and that this loss correlated with reduced tumor necrosis factor alpha (TNF- $\alpha$ ) expression when cultured with epithelial cells. While nonpiliated meningococcal isolates have been previously found in carriage strains, this is the first evidence of an association between type IV pili from meningococci and a proinflammatory epithelial response. We also identified potentially important metabolic differences between carriage and disease isolates, including the sulfate assimilation pathway.
\end{abstract}

IMPORTANCE Neisseria meningitidis causes meningococcal disease but is frequently carried in the throats of healthy individuals; the factors that determine whether invasive disease develops are not completely understood. We carried out detailed studies of isolates, collected from patients and their household contacts, to identify differences between commensal throat isolates and those that caused invasive disease. Though isolates were identical by laboratory typing methods, we uncovered many differences in their genomes, in gene expression, and in their interactions with host cells. In particular, we found that several carriage isolates had lost their type IV pili, a surprising finding since pili are often described as essential for colonization. However, loss of type IV pili correlated with reduced secretion of a proinflammatory cytokine, TNF- $\alpha$, when meningococci were cocultured with human bronchial epithelial

November/December 2017 Volume 2 Issue 6 e00127-17
Received 14 September 2017 Accepted 18 October 2017 Published 14 November 2017 Citation Ren X, Eccles DA, Greig GA,

Clapham J, Wheeler NE, Lindgreen S, Gardner PP, MacKichan JK. 2017. Genomic, transcriptomic, and phenotypic analyses of Neisseria meningitidis isolates from disease patients and their household contacts. mSystems 2:e00127-17. https://doi.org/10 $.1128 / \mathrm{mSystems} .00127-17$.

Editor Peter J. Turnbaugh, G. W. Hooper Research Foundation

Copyright 02017 Ren et al. This is an openaccess article distributed under the terms of the Creative Commons Attribution 4.0 International license.

Address correspondence to Joanna $\mathrm{K}$ MacKichan, joanna.mackichan@vuw.ac.nz.

Disease and carriage meningococcal isolates from NZ household contacts were compared to shed light on carriage and virulence 
cells; hence, the loss of pili could provide an advantage to meningococci, by resulting in a dampened localized host immune response.

KEYWORDS Neisseria meningitidis, type IV pili, carriage, household contact

/eisseria meningitidis is a Gram-negative bacterium and the causative agent of meningococcal disease, which can result in inflammation of the meninges as well as septicemia. This disease is often fatal, even with prompt antibiotic treatment, and often causes long-term sequelae (1). Meningococcal disease can occur sporadically or in epidemics that affect millions of people, especially children, worldwide. Despite the seriousness of the invasive disease, $N$. meningitidis comprises part of the normal nasopharyngeal flora of healthy individuals; host invasion, resulting in septicemia and meningitis, is a relatively rare event. Epidemiology reveals that only a small group of hypervirulent lineages cause most meningococcal disease cases in the world $(2,3)$. From 1991 to 2008, New Zealand experienced a protracted epidemic, with the majority ( $>85 \%)$ of disease cases caused by a single strain type, defined as B:4:P1.7-2,4, belonging to one of two sequence types (STs), ST-42 and ST-154, of the ST-41/44 clonal complex (lineage 3) (4-6). The 2005 introduction of MenNZB, a strain-specific vaccine that elicited protective antibodies to the PorA allele, lowered the number of cases dramatically, until it was discontinued in 2008 (7-10). However, this strain type remains in circulation in New Zealand, having caused $>16 \%(11 / 68)$ of total meningococcal cases in 2013 (11). Carriage studies conducted during the epidemic suggested that the New Zealand epidemic strain type is highly virulent but with low transmissibility (12). The genetic basis of this high virulence has not been identified.

Virulence in $N$. meningitidis is thought to be polygenic, with many different genes contributing to invasive disease (13). Comparing the genomes of large numbers of isolates has not given a clear picture of virulence-related genes, due to the extensive genetic diversity among invasive and carriage strains (14). Previous gene expression studies showed large differences in the transcription repertoire between $N$. meningitidis and the related commensal species Neisseria lactamica (15), as well as between a disease-associated serogroup B strain (MC58) and a carriage-associated strain (alpha710) upon adhesion to human nasopharyngeal cells (13).

Household crowding has been shown to be a major risk factor for meningococcal disease, and household contact with a meningococcal disease patient is associated with increased risk for invasive disease (16-18). During the New Zealand epidemic, a study of carriage rates among household contacts of patients was carried out in Auckland (12). N. meningitidis was cultured from throat swabs from healthy household contacts of an index meningococcal disease patient, within $24 \mathrm{~h}$ of disease notification and prior to administration of chemoprophylaxis to contacts. $N$. meningitidis isolates were collected from patient blood or cerebrospinal fluid (CSF). Of $108 \mathrm{~N}$. meningitidis carriers, $50 \%$ were found to carry the same strain type, as identified by multilocus sequence typing (MLST), serotyping, and porA subtyping, as the index patient from their household (12).

Though host and environmental factors play a role in disease development, we asked whether bacterial differences could have also played a role in disease development. We selected several groups of $N$. meningitidis isolates, derived from single households and in most cases indistinguishable by laboratory typing methods, to identify genetic differences potentially important for disease development. Isolates from one household were investigated in greater detail and were found to differ in their interactions with tissue culture host cells. Whole-genome sequencing and transcriptome analysis methods were used to identify variations. We then investigated potential associations between these variants and phenotypes.

\section{RESULTS}

Whole-genome sequencing of household isolates reveals that they are closely related. During the height of the New Zealand serogroup B meningococcal epidemic, 
TABLE 1 Epidemiological and molecular data associated with households in this study

\begin{tabular}{|c|c|c|c|c|}
\hline Household & Isolate & Sequence type ${ }^{a}$ & Age $^{b}(y r)$ & Site ${ }^{c}$ \\
\hline \multirow[t]{3}{*}{ NZ96/294 } & NZ96/294 & ST-42 & 38 & Blood \\
\hline & NZCM149 & ST -42 & & Throat \\
\hline & NZCM148 & ST-5159 & & Throat \\
\hline \multirow[t]{2}{*}{ NZ97/019 } & NZ97/019 & ST -42 & 1 & CSF \\
\hline & NZCM162 & ST-42 & & Throat \\
\hline \multirow[t]{2}{*}{ NZ97/021 } & NZ97/021 & ST-42 & 3 & CSF \\
\hline & NZCM165 & ST- 42 & & Throat \\
\hline \multirow[t]{5}{*}{ NZ97/133 } & NZ97/133 & ST -42 & 13 & Blood \\
\hline & NZCM132 & ST -42 & & Throat \\
\hline & NZCM133 & ST-42 & & Throat \\
\hline & NZCM134 & ST-42 & & Throat \\
\hline & NZCM135 & ST- 42 & & Throat \\
\hline \multirow[t]{4}{*}{ NZ97/192 } & NZ97/192 & ST-154 & 15 & CSF \\
\hline & NZCM238 & ST-154 & & Throat \\
\hline & NZCM239 & ST-154 & & Throat \\
\hline & NZCM240 & ST-154 & & Throat \\
\hline \multirow[t]{2}{*}{ NZ98/058 } & NZ98/058 & ST-154 & 59 & Blood \\
\hline & NZCM246 & ST-154 & & Throat \\
\hline \multirow[t]{2}{*}{ NZ98/074 } & NZ98/074 & ST-154 & 18 & Blood \\
\hline & NZCM245 & ST-154 & & Throat \\
\hline
\end{tabular}

${ }^{a}$ All isolates, apart from NZCM148, belong to the New Zealand epidemic serogroup B strain type: B:4:P1.7-2,4, ST $41 / 44$ clonal complex (lineage 3).

${ }^{b} \mathrm{Age}$ of the index patient.

cSite where the meningococcal isolate was collected.

a study was carried out to assess the prevalence of carriage among household contacts of patients with meningococcal disease (12). A total of 954 contacts of 160 patients agreed to nasopharyngeal swabs; 196 of these contacts (20.5\%) were found to be carriers. Isolates from 51 households, including those from healthy subjects and from patients, were subjected to routine MLST and serotyping, revealing that 29 of the 51 households had at least one healthy individual carrying a meningococcal isolate indistinguishable from the invasive isolate from the patient. MLST, based on the nucleotide sequence of 7 housekeeping genes, does not give a full picture of the genetic diversity of the isolates. To address this, we selected 21 isolates, from 7 households, to analyze further with shotgun whole-genome sequencing using Illumina Miseq technology. The index meningococcal isolates for all 7 households were of the New Zealand epidemic type, with three belonging to ST-154 and four belonging to ST-42 (Table 1). We included one carriage isolate, NZCM148, from the NZ96/294 household that was clearly distinct from the index patient isolate. It was included to test whether isolates within the same household share similarities regardless of sequence type. Maximum-likelihood phylogeny showed isolates separated into two major groups, corresponding to their sequence type (ST-42 versus ST-154). In addition, isolates from the same household clustered together, even within a shared sequence type (Fig. 1). Within the NZ96/294 household, carriage isolate NZCM148 belongs to a different clonal complex and did not cluster with the index disease-associated isolate.

Whole-genome sequencing identified variations among household isolates. We extracted high-confidence variants between the household isolates (excluding the unrelated isolate NZCM148) and the New Zealand epidemic strain reference genome, NC_017518.1, and removed variants that were common to all isolates within a household group, variants within the pils antigenic variation region, and predicted tandem repeats. Within the same household, the number of variants among isolates ranged from 9 to 210 (Table 2).

We annotated the variants according to their location and possible effect on coding sequences (CDSs) using SnpEff (19). For ease of annotation, variants were analyzed in 


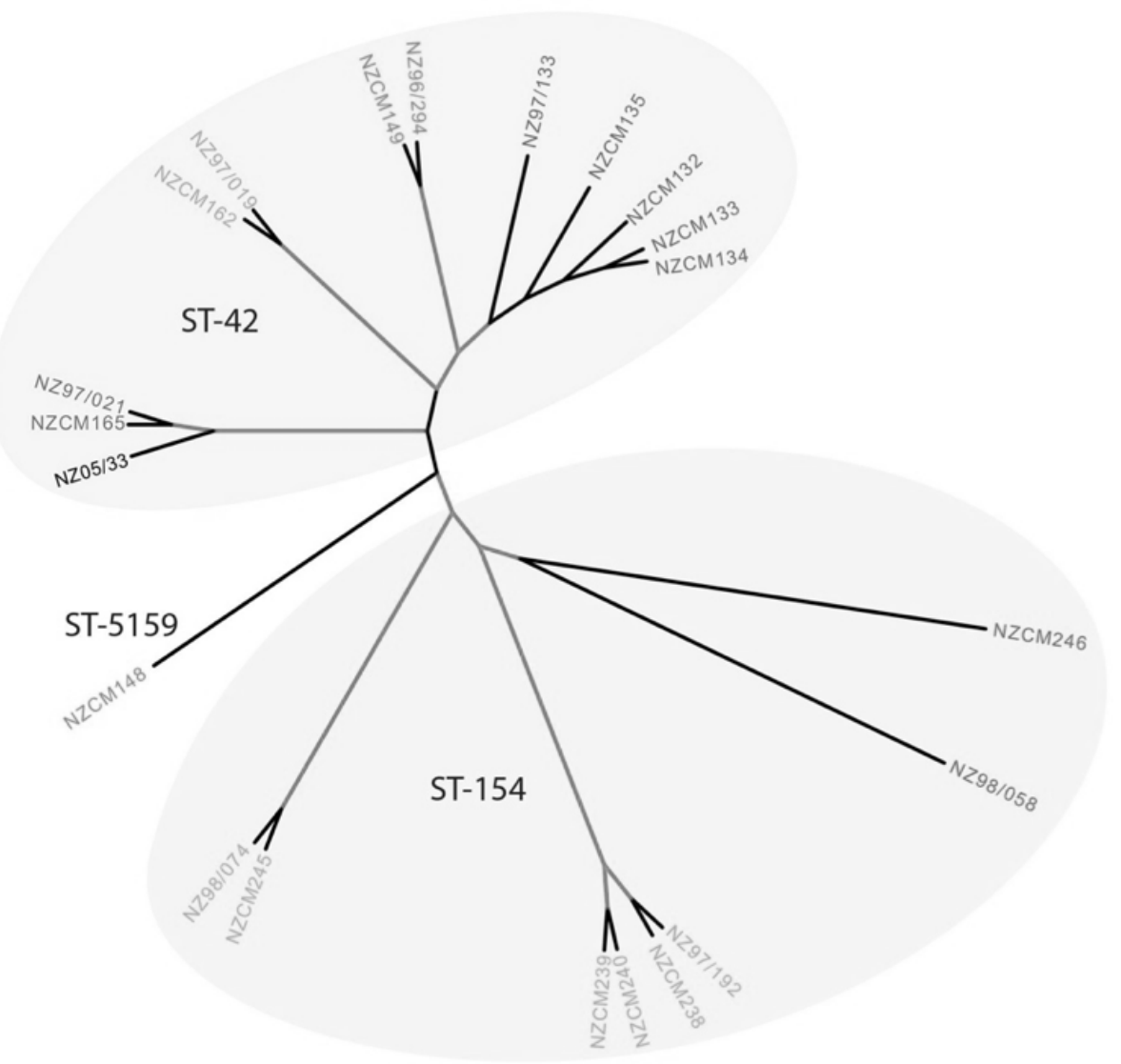

FIG 1 Maximum-likelihood phylogeny of the household isolates from core SNPs. A phylogeny was constructed based on core SNP differences between each isolate and the reference genome of $N$. meningitidis NZ-05/33 (NC_017518.1). A proportional cladogram is presented so that short branches are visible. Branches with greater than $90 \%(200 \times$ bootstrap) support are highlighted in red. Isolates from the same household are shown in the same color. Two ovals indicate the sequence types of the isolates within the two major branches. Isolate NZCM148 from household NZ96/294 belongs to a different clonal complex.

"clumped" format, where variants close to each other were presented as one change. This method ensures that changes within a codon are annotated correctly. There were a total of 421 clumped variants; of these, 141 were classified as intergenic (defined as more than 100 bp away from an annotated transcript), 19 were located less than $100 \mathrm{bp}$

TABLE 2 Number of variants among the household isolates and their classification ${ }^{o}$

\begin{tabular}{|c|c|c|c|c|c|c|c|c|c|c|c|}
\hline \multirow[b]{2}{*}{ Household } & \multirow{2}{*}{$\begin{array}{l}\text { No. of } \\
\text { isolates in } \\
\text { household }\end{array}$} & \multirow{2}{*}{$\begin{array}{l}\text { Total no. } \\
\text { of variants } \\
\text { (NC_017518.1) }\end{array}$} & \multicolumn{9}{|c|}{ No. of variants by type ${ }^{b}$ : } \\
\hline & & & 1 & U & D & $\mathbf{S}$ & In & De & M & $\mathbf{F}$ & Stop \\
\hline NZ96/294 & 3 & 23 & 6 & 6 & 5 & 2 & 0 & 0 & 2 & 2 & 0 \\
\hline NZ97/019 & 2 & 210 & 28 & 20 & 19 & 99 & 0 & 2 & 37 & 5 & 0 \\
\hline NZ97/021 & 2 & 9 & 2 & 2 & 1 & 1 & 1 & 0 & 1 & 1 & 0 \\
\hline NZ97/133 & 5 & 51 & 20 & 4 & 4 & 6 & 2 & 2 & 5 & 8 & 0 \\
\hline NZ97/192 & 4 & 176 & 78 & 9 & 3 & 27 & 3 & 4 & 35 & 16 & 1 \\
\hline NZ98/058 & 2 & 97 & 37 & 1 & 9 & 17 & 3 & 1 & 21 & 7 & 1 \\
\hline NZ98/074 & 2 & 44 & 8 & 2 & 0 & 12 & 0 & 0 & 13 & 9 & 0 \\
\hline
\end{tabular}

The single nucleotide polymorphisms detected are listed, including information about the household group where they were identified, number of isolates in each household, and summary of the type of polymorphism. The divergent isolate NZCM148 has been omitted.

${ }^{b}$ Abbreviations: I, intergenic; U, upstream; D, downstream; S, synonymous; In, in-frame insertions; De, in-

frame deletions; $M$, missense; $F$, frameshift; Stop, gained stop codon. 
TABLE 3 Homopolymeric and small-repeat variants upstream and within annotated transcripts

\begin{tabular}{|c|c|c|c|c|c|}
\hline Position & $\begin{array}{l}\text { Reference } \\
\text { sequence }\end{array}$ & $\begin{array}{l}\text { Alternative } \\
\text { sequence(s) }\end{array}$ & Location/effect & Locus & Change $^{e}$ \\
\hline $30823^{a}$ & $G(A) 11 C$ & $G(A) 10 C ; G(A) 12 C$ & Upstream & NMBNZ0533_0037 & \\
\hline $45680^{b}$ & $C(G) 9 C$ & $C(G) 10 C$ & Frameshift & NMBNZ0533_0050 & \\
\hline $75462^{a}$ & C(T)9G & $\mathrm{C}(\mathrm{T}) 10 \mathrm{G}$ & Frameshift & $s i a D$ & Lys $142 \mathrm{fs}$ \\
\hline $75798^{a}$ & $\mathrm{~T}(\mathrm{G}) 7 \mathrm{~T}$ & $\mathrm{~T}(\mathrm{G}) 6 \mathrm{~T}$ & Frameshift & $\operatorname{siaD}$ & Thr30fs \\
\hline $75872^{a}$ & $C(T) 7 A$ & $\mathrm{C}(\mathrm{T}) 6 \mathrm{~A}$ & Frameshift & siaD & lle5fs \\
\hline $338414^{a}$ & $A(G) 9 C$ & $A(G) 10 C$ & Frameshift & NMBNZ0533_0325 & Pro958fs \\
\hline 354493 & CAG(10) & CGAGA(G)7A & Upstream & fet $A$ & \\
\hline $388239^{a}$ & $C(G) 9 C$ & $\begin{array}{l}\mathrm{C}(\mathrm{G}) 10 \mathrm{C} ; \\
\mathrm{C(G)11 \textrm {C }}\end{array}$ & Frameshift & NMBNZ0533_0355 & Pro96fs \\
\hline $424181^{a}$ & $C(G) 8 A$ & $C(G) 9 A ; C(G) 7 A$ & Frameshift & $\lg t A$ & Glu88fs \\
\hline 506007 & $G(C) 12 G$ & $\mathrm{G}(\mathrm{C}) 11 \mathrm{G}$ & Frameshift & NMBNZ0533_0475 & \\
\hline 515698 & $C(G) 11 \mathrm{~A}$ & $\begin{array}{l}C(G) 10 A ; C(G) 12 A ; \\
C(G) 13 A\end{array}$ & Frameshift & NMBNZ0533_0485 & Glu63fs \\
\hline 546125 & $C(G) 9 A$ & $C(G) 11 A$ & Frameshift & NMBNZ0533_0508 & Pro164fs \\
\hline $862493^{c}$ & $A(G) 8 C$ & $A(G) 7 C$ & Upstream & purC & \\
\hline 931392 & $C(G) 9 A$ & $C(G) 10 A ; C(G) 11 A$ & Frameshift & NMBNZ0533_0877 & Ter193fs \\
\hline $1119938^{d}$ & $C(G) 11 A$ & $\begin{array}{c}C(G) 7 A ; C(G) 10 A ; \\
C(G) 8 A\end{array}$ & Upstream & opcA & \\
\hline 1257866 & $C(G) 9 A$ & $\begin{array}{c}C(G) 8 A ; C(G) 7 A ; \\
C(G) 10 A\end{array}$ & Frameshift & NMBNZ0533_1221 & Gly105fs; Phe106fs \\
\hline $1472851^{a}$ & $\mathrm{G}(\mathrm{T}) 7 \mathrm{G}$ & $\mathrm{G}(\mathrm{T}) 8 \mathrm{G}$ & Frameshift & $m s b B$ & Lys $143 \mathrm{fs}$ \\
\hline $1487249^{\circ}$ & $A(C) 12 G$ & $A(C) 11 G ; A(C) 10 G$ & Upstream & porA & \\
\hline 1529640 & G(GAAGA)8G & $\begin{array}{l}\text { G(GAAGA)7G; G(GAAGA)9G; } \\
\text { G(GAAGA)10G }\end{array}$ & Upstream & NMBNZ0533_1444 & \\
\hline 1598967 & $\mathrm{C}(\mathrm{TGCT}) 7 \mathrm{~T}$ & $\mathrm{C}(\mathrm{TGCT}) 5 \mathrm{~T}$ & Frameshift & NMBNZ0533_1501 & Glu4fs \\
\hline $1669031^{c}$ & $\mathrm{C}(\mathrm{G}) 8 \mathrm{~T}$ & $\mathrm{C}(\mathrm{G}) 9 \mathrm{~T}$ & Frameshift & NMBNZ0533_1559 & Val49fs \\
\hline 1721245 & C(CTTCT)4C & $\begin{array}{l}\text { С(CTाCT)7C; } \\
\text { С(СTCT)9C }\end{array}$ & Upstream & NMBNZ0533_1610 & \\
\hline 1963892 & $C(A) 5 C$ & $C(A) 6 C ;(A) 6 C$ & Upstream & NMBNZ0533_1807 & \\
\hline 1997634 & $A(C) 7 G$ & $\begin{array}{c}A(C) 8 G ; A(C) 9 G ; \\
A(C) 11 G\end{array}$ & Frameshift & NMBNZ0533_1834 & Gly 141 fs \\
\hline
\end{tabular}

${ }^{a}$ Confirmed by Sanger sequencing within the NZ97/192 household isolates.

${ }^{b}$ Multiple PCR products.

'Mixed sequences detected by Sanger sequencing within the NZ97/192 household isolates.

${ }^{a}$ Not confirmed by Sanger sequencing within the NZ97/192 household isolates.

efs, frameshift.

downstream of an annotated transcript, and 40 were located less than 100 bp upstream of an annotated transcript. Within coding regions, 90 variants caused synonymous changes, 83 caused missense changes, and 38 were predicted to cause frameshifts in the coding region, with 10 in-frame insertions and 11 in-frame deletions (dels) also identified (summarized in Table S1 in the supplemental material). Many variants that were predicted to be upstream of coding sequences (17/40) or to cause frameshifts (27/38) were within homopolymeric and small-repeat sequences (Table 3). As variants within homopolymeric sequences are difficult to assess using whole-genome sequencing data, we confirmed some of these differences for the NZ97/192 family using Sanger sequencing (details in Table 3). Differences within homopolymeric sequences may indicate that these transcripts are under phase-variable regulation. Indeed, variants were found within genes that are known to be phase variable, including $\lg t A$, siaD, pilC, por $A$, and those encoding opacity proteins. Interestingly, variants that were predicted to affect siaD translation were detected only in carriage isolates ( 3 isolates from two households). These three carriage isolates (NZCM238, NZCM240, and NZCM245) all had variants in single nucleotide repeat sequences that resulted in a translational frameshift of siaD. The presence of detectable capsule in these isolates was tested by slide agglutination. NZCM238 and NZCM245 were found to be nongroupable, confirming loss of the capsule, although results could not be determined for NZCM240, which exhibited extensive autoagglutination. Serogroup B capsule expression was confirmed by slide agglutination for the other isolates from the household, NZ97/192 and NZCM239. Other variants were detected in isolates from both patients and carriers. 
Six variants were found that restored open reading frames (ORFs) in orthologs of NMBNZ0533_0050 (pilC1), NMBNZ0533_0475 (pilC1), and NMBNZ0533_1834 (Dca), which are annotated as pseudogenes and are predicted to be degenerate in the reference genome.

Of the 94 variants that were found to cause amino acid substitutions, insertions, or deletions, only 5 were predicted to alter protein function by PROVEAN (Protein Variant Effect Analyzer) (20, 21). These changes were found in NMBNZ0533_0397 (IgtC), NMBNZ0533_1030 (chloride transporter), NMNZ0533_1646 (hmbR), NMBNZ0533_1790 (tbpA), and NMNZ0533_1997 (citrate transporter family protein). IgtC encodes a glycosyltransferase that modifies lipo-oligosaccharide, and a Gly57 deletion mutation was found in all of the NZ97/192 household isolates. hmbR encodes a TonB-dependent hemoglobin receptor, a potential virulence factor involved in iron acquisition. The mutation Leu380dup was found in a carriage-associated isolate, NZCM135. tbpA has also been shown to be involved in iron acquisition. The mutation in tbpA (Gly795Ser) was found in NZCM134, a carriage-associated isolate. The NMBNZ0533_1030 gene encodes a chloride transporter protein, and a variant in this gene was found in several families and in both carriage- and disease-associated isolates. The NMBNZ0533_1997 gene encodes a citrate transporter protein, and the mutation Ala263del was identified in NZCM134, an isolate from a healthy carrier.

A large number of single nucleotide polymorphisms (SNPs) were identified in the invasive isolate NZ97/019 in an approximately 5-kb region (Table S1), suggesting the possibility of a recombination event with another isolate or species. To investigate the origin of this DNA region, the NZ97/019 draft genome was aligned with the reference genome, NZ-05/33. The sequence corresponding to bp 185000 to 190142 was extracted and searched against the PubMLST Neisseria database (22). For the six genes contained in this region, four from NZ97/019 had alleles that matched those found in an isolate of a related, carriage-associated resident of the nasopharynx, Neisseria bergeri. The top hits for the remaining two genes were also $N$. bergeri-associated alleles, although these were not identical. When the corresponding sequence from the reference genome, NZ-05/33, was queried in the database, all the alleles matched $N$. meningitidis isolates only. The most likely explanation for these observations is that the New Zealand epidemic strain acquired $\sim 5 \mathrm{~kb}$ of DNA from a commensal neisserial strain while in the nasopharynx. N. bergeri is a newly proposed species, which was formerly considered a divergent strain of $N$. polysaccharea $(23,24) . N$. polysaccharea and $N$. bergeri are both commensal spècies, closely related to $N$. meningitidis, which reside in the nasopharyngeal mucosa. The exchange of DNA between $N$. meningitidis and other bacterial members of the nasopharyngeal microbiome has previously been documented $(25,26)$. We did not find any evidence that the associated throat isolate from the NZ97/019 household (NZCM162) had acquired DNA through recombination.

No consistent patterns were identified that could distinguish isolates from healthy carriers from those responsible for invasive disease, but many genomic differences were observed among isolates from the same household. Many of these genomic differences could result in altered transcription, with these differences underpinning different outcomes in the host. We therefore selected four isolates from the NZ97/192 household group for further phenotypic and transcriptome analyses.

Household isolates do not differ significantly for cell association or invasion of bronchial epithelial cells. The ability to adhere to respiratory epithelial cells is essential for the initial colonization of the nasopharynx by meningococci. We measured the ability of our isolates to associate with $16 \mathrm{HBE}$ epithelial cells, an immortalized normal human bronchial epithelial cell line (27). Mid-log-phase bacteria were cocultured with cells, followed by washing and enumeration of cell-associated bacteria (adhered and intraceflular). CFU recovered were normalized to the initial inoculant, also estimated by CFU counts. We found high variability in the ability of meningococcal isolates to adhere, but no statistically significant differences among the isolates. Numbers of cellassociated bacteria for the NZCM238 isolate were slightly higher than those for the other isolates, but this difference was not significant (Fig. 2A). 

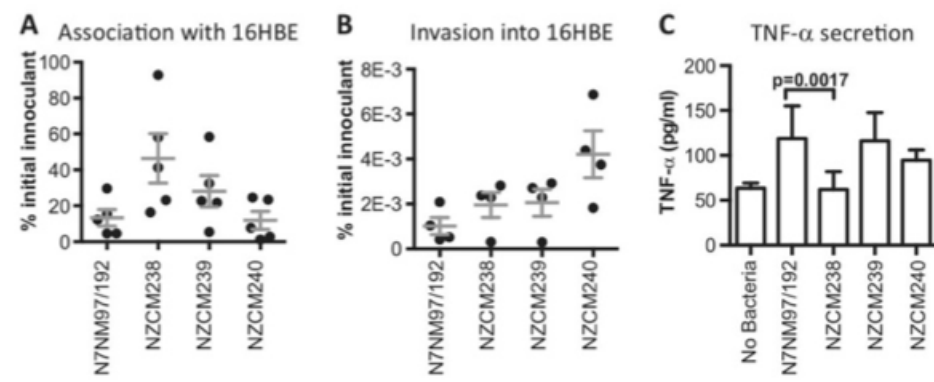

FIG 2 Isolates from the NZ97/192 household interact differently with host respiratory epithelial 16HBE cells. For each assay, all isolates were tested together and in biological replicates. Each assay was performed in triplicate at least twice. (A) Viable bacteria associated with the 16HBE cells (adhered and intracellular), expressed as a percentage of the initial inoculant. Horizontal bars indicate means, while error bars indicate standard deviations. (B) Viable bacteria present inside $16 \mathrm{HBE}$ cells after gentamicin treatment, expressed as a percentage of the initial inoculant. Horizontal bars indicate means, while error bars indicate standard deviations. (C) Concentration of TNF- $\alpha$ in the culture media of 16HBE cells and bacterial cocultures (picograms per milliliter, $n=6$ ). The average value is plotted for each isolate, and error bars indicate standard deviations.

The ability to invade respiratory epithelial cells may render the bacteria invisible to immune surveillance and may also contribute to invasive disease development. We measured the isolates' ability to invade $16 \mathrm{HBE}$ cells using a gentamicin protection assay and enumeration of intracellular bacteria. Invasion rates were low among all four isolates, and no statistically significant differences were seen. NZCM240 was slightly more invasive in this assay, but this did not reach statistical significance (Fig. 2B).

Isolates differ in their ability to elicit TNF- $\alpha$ secretion in bronchial epithelial cells. Tumor necrosis factor alpha (TNF- $\alpha$ ) is a major proinflammatory cytokine induced during meningococcal infection, by monocytic cells (systemic) or epithelial or endothelial cells (locally). The levels of TNF- $\alpha$ in the blood positively correlate with disease severity, with higher levels of TNF- $\alpha$ corresponding to more severe disease (28). TNF- $\alpha$ expression in the mucosal epithelium in response to pathogenic neisseriae results in the efficient recruitment and activation of neutrophils $(29,30)$. We therefore infected synchronized $16 \mathrm{HBE}$ cultures with the different isolates and measured the amount of TNF- $\alpha$ in the culture medium after $24 \mathrm{~h}$ (Fig. 2C). The NZ97/192-infected 16HBE cells secreted the largest amount of TNF- $\alpha(118.8 \pm 36 \mathrm{pg} / \mathrm{ml}$ [standard deviation \{SD\}], $n=$ 6), though TNF- $\alpha$ levels in NZCM239-infected cultures (116.1 $\pm 31.5 \mathrm{pg} / \mathrm{ml}, \mathrm{SD}, n=5$ ) were similar. The NZCM238-infected cultures had significantly smaller amounts of TNF- $\alpha(62.0 \pm 20.0 \mathrm{pg} / \mathrm{ml}, \mathrm{SD}, n=6)$ than cultures infected with other isolates from within the household group. The level detected was similar to that observed in uninfected cultures $(63.5 \pm 5.7 \mathrm{pg} / \mathrm{ml}, n=5)$. NZCM240 induced slightly lower levels than NZ97/192 and NZCM239, though this was not statistically significant. These assays demonstrated subtle phenotypic differences between these highly related isolates in their interaction with host epithelial cells.

NZ97/192 household isolates have different transcription profiles in the presence of respiratory epithelial cells. We demonstrated that the isolates within the NZ97/192 household group interact differently with 16HBE cells, especially with the reduced ability of one isolate, NZCM238, to induce TNF- $\alpha$ secretion from $16 \mathrm{HBE}$ cells after infection. To understand how the transcriptional response of the isolates varies in response to $16 \mathrm{HBE}$ cells, we purified total RNAs from bacteria during mid-log phase while in coculture with $16 \mathrm{HBE}$ cells and compared their transcription profiles. For this analysis, we used the annotated RefSeq genome NMBNZ0533 as our reference. A total of 37 transcripts, excluding rRNA, were significantly different (adjusted $P \leq 0.05$ as determined by DESeq) between NZ97/192 and one of the three carriage-associated isolates (Table S2) (31). Most of the genes were downregulated in the carriageassociated isolates relative to the disease-associated isolate. We also analyzed the 


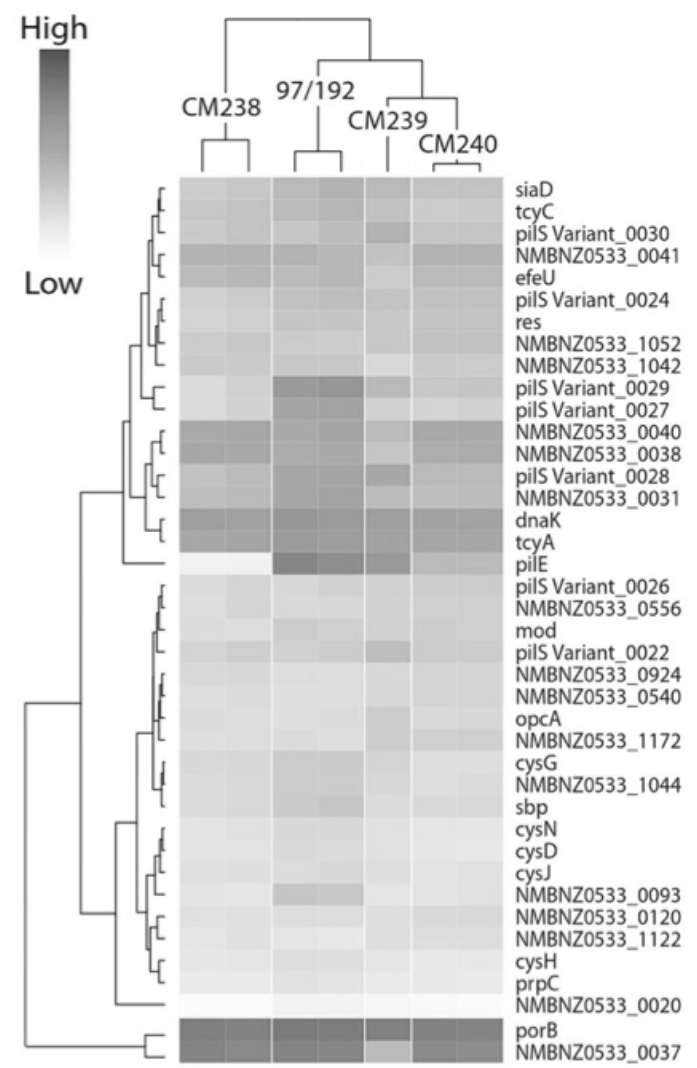

FIG 3 Heat map and dendrogram of differentially expressed transcripts among the NZ97/192 household isolates. Transcripts that were found to be significantly different by DESeq $\left(-1 \leq \log _{2} \Delta \geq 1\right.$; adjusted $P$ $<0.05$ ) are displayed. Dark blue represents high levels of expression. The dendrogram illustrates the clustering of replicate samples for a given isolate. For detailed transcript information, see Table S2.

replicates separately and found that most transcripts were confirmed in the replicate data set. A heat map of 37 transcripts with the largest differences $\left(-\log _{2} \geq \pm 1\right.$, adjusted $P \leq 0.05$ ) from both replicates is displayed in Fig. 3, which demonstrates that the replicates cluster together. In addition, NZCM238 clustered separately from the other isolates. One of the genes with the largest difference in expression is pilE, the expressed pilin gene, with almost no expression detected in NZCM238.

We annotated the predicted gene products according to predicted cellular location and function using the UniProt database (32) (Fig. 4A and B). Additional localization information from PSORTb and NeMeSys is summarized in Table S2 $(33,34)$. One transcript was predicted to contain a tRNA (tRNA-Leu), while the remaining transcripts were predicted to encode intact or degenerate proteins. Subcellular location was not known for seven proteins, while six proteins were predicted to be located in either the periplasm or the outer membrane. Eight proteins were predicted to be associated with the pilus, and 15 proteins and the tRNA were predicted to be cytoplasmic. Functional prediction resulted in 10 genes with unclassified function; these included four transcripts missing from the UniProt database because they were not predicted to encode functional proteins (NMBNZ0533_0020, NMBNZ0533_0040, NMBNZ0533_1052, and NMBNZ0533_1044), one predicted to contain an RNA molecule (NMBNZ0533_1172, tRNA-Leu), and five with unknown function. UniProt predicted that six transcripts encode proteins involved in metabolic pathways, including four genes (cys $N$, cys $D$, cys), and $(\mathrm{cys} H$ ) that encode enzymes in the sulfate assimilation pathway. In addition to the 
A

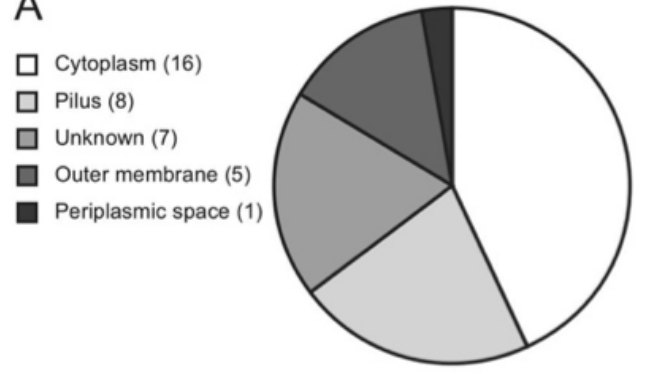

B

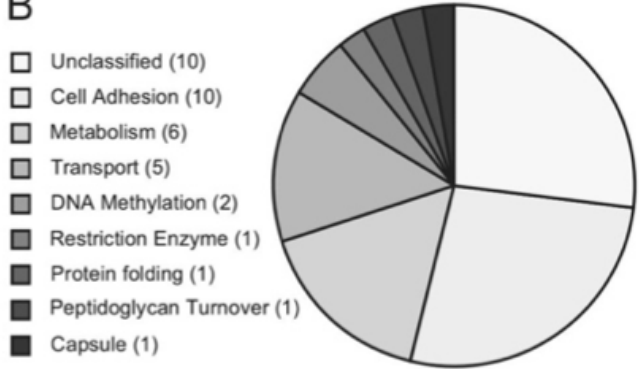

C

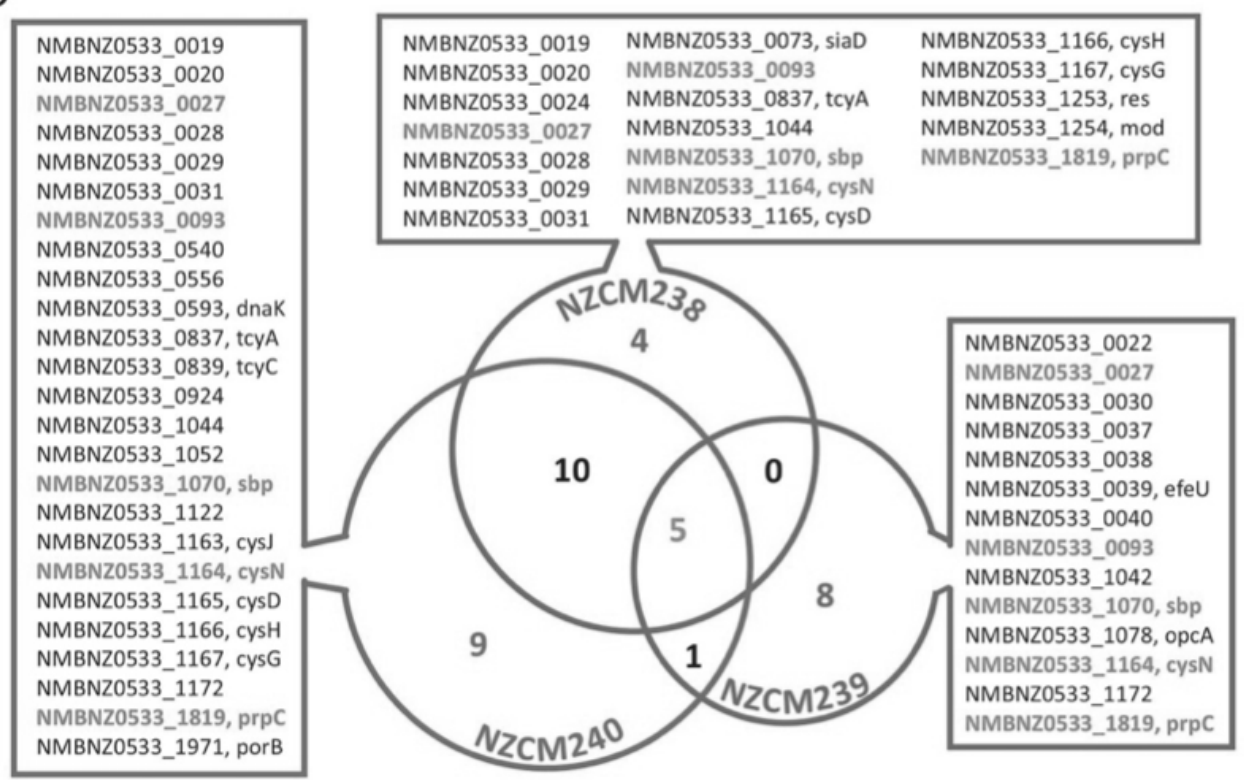

FIG 4 Predicted location and functional classification of differentially expressed transcripts. Transcripts were classified according to the subcellular location and predicted function of their encoded protein product, using the UniProt database. (A) Cellular location analysis shows that 16/37 transcripts were predicted to encode cytoplasmic proteins. (B) The function of 10 out of 37 transcript gene products could not be classified, either because they were absent from the UniProt database or because their function was unknown. Six transcripts encoded proteins predicted to be involved in metabolism, highlighting possible metabolic differences between diseaseand carriage-associated isolates. (C) Venn diagram listing differentially expressed ( $\log _{2} \Delta \geq \pm 1$; adjusted $P<0.05$ ) transcripts between each carriage isolate and the disease isolate (NZ97/192). Five transcripts, highlighted in red, were differentially expressed in all three carriage isolates, relative to the invasive isolate. For detailed transcript classification, see Table S2.

genes located within the pilin variation locus, an additional transcript, a putative hemagglutinin, was also predicted to function in cell adhesion. Although OpcA was not predicted by UniProt to have a function in adhesion, we classified it as an adhesin, based on previous reports (35-38). Five proteins were predicted to function as transporters; the gene for one of these encodes a sulfate binding protein, which also functions in the sulfate assimilation pathway. PorB, a major outer membrane porin, which has been shown to translocate to the host cell mitochondrion, where it prevents apoptosis, was also classified as a transporter (39-41). Other transcripts were predicted to function in DNA methylation (mod and NMBNZ0533_0924), capsule biosynthesis $(s i a D)$, protein folding (dnaK), peptidoglycan turnover (MltA family protein), and restriction modification (res).

A Venn diagram and a list of the transcripts with significantly different expression (adjusted $P \leq 0.05$ ) and their respective isolates are pictured in Fig. 4C. Four transcripts 
were uniquely different between NZ97/192 and NZCM238, while 8 transcripts were uniquely different between NZ97/192 and NZCM239 and 9 transcripts were uniquely different between NZ97/192 and NZCM240. Among these transcripts, 5 had decreased expression in all three carriage-associated isolates (red in Fig. 4C).

One of the 5 transcripts with decreased expression in all three carriage isolates was NMBNZ0533_0027, part of the pilS antigenic region. NMBNZ0533_0093 encodes a conserved hypothetical protein with a predicted outer membrane protein domain. This expression difference was confirmed by real-time quantitative PCR (qRT-PCR), where its expression was almost undetectable in the carriage isolates ( $>90 \%$ reduction, relative to invasive disease isolate, $P<0.0001, n=9$ ). Interestingly, $241 \mathrm{bp}$ upstream of the predicted translation start site, there is a stretch of C's that differed in number between NZ97/192 and all three isolates from healthy carriers. NZ97/192 contained 9 C's, whereas NZCM238, NZCM239, and NZCM240 all had 10 C's (confirmed by Sanger sequencing). This configuration is characteristic of genes that undergo phase variation, but it is not known whether this stretch of C's contributes to transcriptional regulation. A second gene, NMBNZ0533_0037, may also undergo phase variation; a homopolymeric sequence of $A$ 's is found $47 \mathrm{bp}$ upstream of the translation start site. The NZ97/192, NZCM238, and NZCM240 isolates all have $10 \mathrm{~A}$ 's in this sequence, while NZCM239, which has significantly reduced expression of the gene, has 11 A's. We were not able to confirm the difference in expression of NMBNZ0533_0037 in the isolates by qRT-PCR, due to extreme variability between the replicates. This may be due to stochastic switches occurring in the tested populations. NMBNZ0533_0037 has an ortholog in the $N$. meningitidis MC58 genome, NMB0032, which shares the same upstream sequence and homopolymeric A tract as NMBNZ0533_0037. NMB0032 has previously been identified as a likely "moderate" phase-variable gene candidate in the study carried out by Siena et al., although they did not find direct evidence of phase variation of this gene (42). However, we did observe differences in the numbers of A's in our isolates (Table 3). NMBNZ0533_0093 has not, to our knowledge, been identified as a phase-variable gene elsewhere. Like many phase-variable genes, NMBNZ0533 0037 encodes a protein that is predicted to be membrane associated, and so it may be subject to selective pressure to evade an immune response $(42,43)$.

Three of the transcripts that are downregulated in all the carriage isolates ( $s b p$, NMBNZ0533_1070; cysN, NMBNZ0533_1164; and cysG, NMBNZ0533_1167), encode proteins predicted to function in the sulfate assimilation pathway. sbp encodes a sulfate binding protein, $c y s N$ encodes the large subunit of a sulfate adenylyltransferase, and cys $G$ encodes a siroheme synthase. For sbp, qRT-PCR confirmed reduced expression in NZCM238 and NZCM239 but not in NZCM240 (NZCM238, 55.6\% $\pm 27.8 \%$ reduction, standard error of the mean [SEM], unpaired $t$ test, $n=10, P=0.023$; NZCM239, $54.6 \% \pm 16.2 \%$ reduction, SEM, unpaired $t$ test, $n=10, P=0.0075$; NZCM240, $26.7 \% \pm$ $22 \%$, reduction, SEM, unpaired $t$ test, $n=10, P=0.105$ ). However, qRT-PCR did not confirm the differences in transcription for $c y s N$ or for $c y s G$. The high variability in the qRT-PCR experiment for $s b p$ was mainly due to two sets of experiments where the level of expression was higher in the carriage isolates than in NZ97/192. The last transcript, prpC (NMBNZ0533_1819), encodes 2-methylcitrate synthase in the citrate synthesis pathway.

In addition to examining the expression differences, we also compared the sequences of the transcripts and predicted protein sequences from the isolates to identify variations, with potential functional consequences, by comparing predicted mutations to the Pfam database and protein orthologs. The only protein that was predicted to be functionally different between all three carriage-associated isolates, compared to NZ97/ 192, was a conserved hypothetical protein encoded by NMBNZ0533_0823. This gene encodes a small protein with a putative hemolysin domain. However, this predicted open reading frame (ORF) was of low quality and, like many of the transcripts predicted to have deleterious frameshift mutations, was not highly expressed under our assay conditions. 


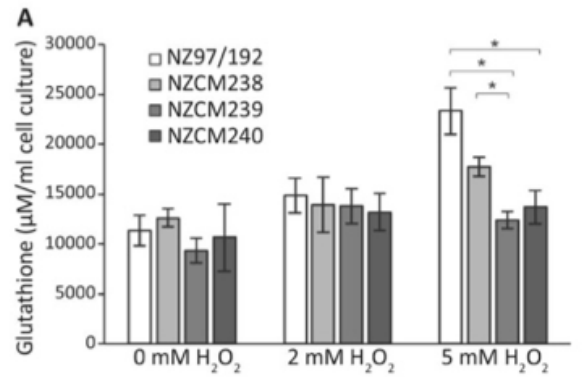

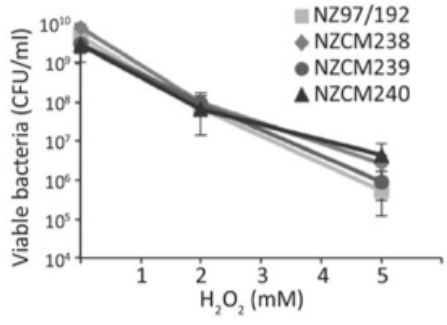

FIG 5 The invasive isolate has higher levels of intracellular glutathione following exposure to oxidative stress. (A) The decreased expression of sulfate assimilation genes in the carriage-associated isolates from the NZ97/192 household leads to lower levels of intracellular glutathione following exposure to $5 \mathrm{mM}$ hydrogen peroxide. ${ }^{*}, P<0.05$. (B) No significant difference was seen in survival rates in the presence of 2 or $5 \mathrm{mM}$ hydrogen peroxide for $20 \mathrm{~min}$. Error bars represent standard deviations.

Intracellular glutathione levels differ between throat and blood isolates following oxidative stress. The expression differed between NZ97/192 household isolates for multiple genes predicted to play a role in sulfate assimilation, including the sulfate binding protein, $s b p$, and a cystine $A B C$ transporter, tcyA, as well as cys $G, c y s H$, $c y s J$, and $c y s N$. The products of the last four genes enable reduction of sulfate into hydrogen sulfide (33). The importance of sulfur metabolism for nasopharyngeal colonization has been previously noted for meningococci (44). Sulfur acquisition via the cysteine biosynthesis pathway is also essential for production of glutathione, a potentially important cellular antioxidant. Glutathione reduces oxidative stress by facilitating reduction of other proteins via cysteine thiol-disulfide exchange (45). To look for functional implications of this differential gene expression, we investigated levels of intracellular glutathione in isolates from the NZ97/192 household. Under normal conditions, glutathione levels were similar between all the isolates (Fig. 5A). However, following exposure to oxidative stress, induced by the addition of $5 \mathrm{mM}$ hydrogen peroxide, significantly higher levels of intracellular glutathione were detected in NZ97/ 192 than in the carriage-associated isolates NZCM239 and NZCM240 $(P<0.05)$. Glutathione levels in NZ97/192 were also higher than those in NZCM238, although they did not reach statistical significance. Higher levels of glutathione are consistent with the higher expression of sulfur acquisition genes in NZ97/192. Under the conditions tested, however, we did not detect any difference in the ability of the isolates to survive hydrogen peroxide challenge for $20 \mathrm{~min}$ (Fig. 5B).

T4P are associated with TNF- $\alpha$ secretion from respiratory epithelial cells. De novo assemblies of NZ97/192, NZCM238, NZCM239, and NZCM240 predicted deletions within the pilS antigenic region in the three carriage-associated isolates compared to NZ97/192 (Fig. 6A), a finding consistent with reduced or absent transcription of pilE in the carriage isolates. To further characterize the pilin locus, we performed PCR against genomic DNA, using primers to transcripts surrounding the pilS region, and identified significant deletions in several isolates (Fig. 6B). PCR against genomic DNA from NZ97/192 yielded a 7-kb fragment, while NZCM238, NZCM239, and NZCM240 yielded fragment sizes of $4 \mathrm{~kb}, 4.5 \mathrm{~kb}$, and $5.6 \mathrm{~kb}$, respectively. The PCR fragment sizes were consistent with those predicted by genome assembly (Fig. 6A). As mentioned above, transcriptome analysis demonstrated that pilE, which encodes the major pilin subunit of type IV pili (T4P), and three other pilS open reading frames were downregulated in carriage-associated isolates. NZCM238 had the lowest expression of pilE, with a $\log _{2}$ fold change of -10.72 . NZCM240 had the second lowest expression $\left(\log _{2}=-3.74\right)$, followed by NZCM239 $\left(\log _{2}=-1.24\right)$. We verified this difference using qRT-PCR, which showed no detectable expression $(n=6)$ in NZCM238, 71\% $( \pm 17 \%$, unpaired $t$ test, $P=$ $0.000164, n=6)$ reduction in NZCM239, and $94 \%$ ( $\pm 3 \%$, unpaired $t$ test, $P<0.0001$, 
A

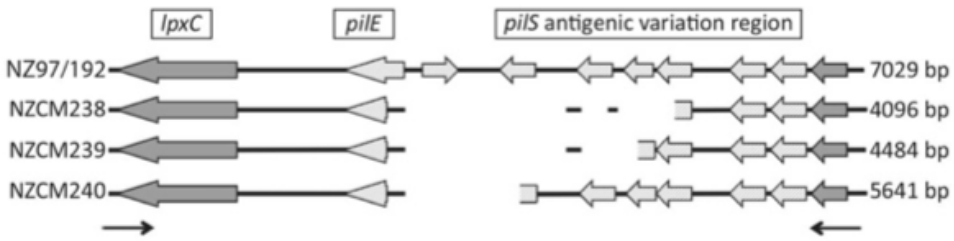

B

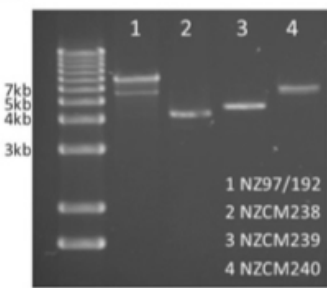

C

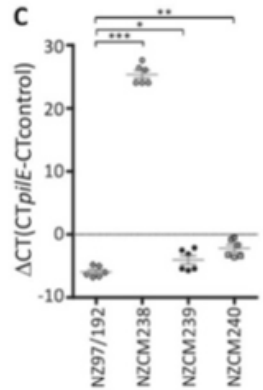

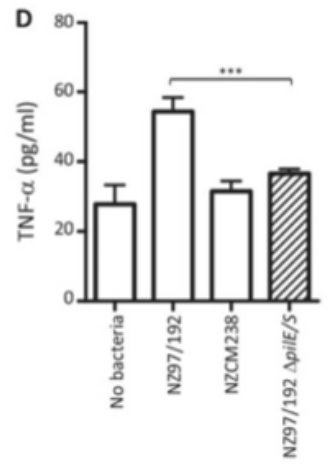

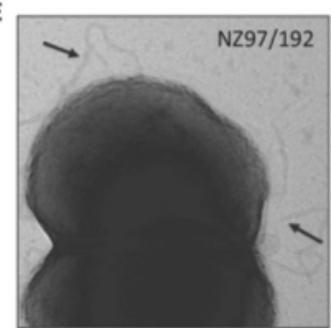

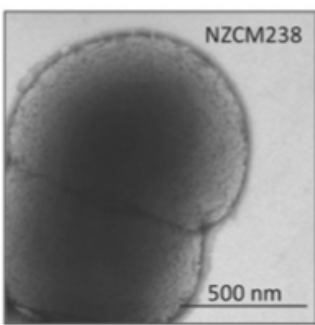

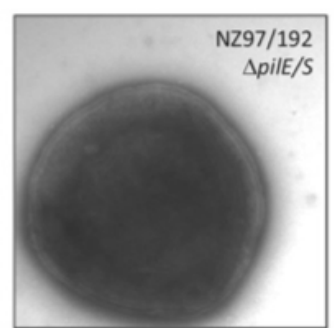

FIG 6 Lack of pilE expression in NZCM238 is associated with lower TNF- $\alpha$ secretion by $16 \mathrm{HBE}$ cells during coculture. (A) De novo assembly, annotation, and alignment of the pilE-pils region of the isolates within the NZ97/192 household revealed a large amount of variability. Deletions of 2,933, 2,545, and 1,388 bp were detected in NZCM238, NZCM239, and NZCM240, respectively, compared to the index strain, NZ97/192. Block arrows depict predicted open reading frames. Small arrows below the drawing indicate primers used for genomic PCR. (B) Genomic PCR confirmed the length of the pilE-pils region in each isolate, using primers indicated above. (C) Quantitative reverse transcriptase PCR (qRT-PCR) confirmation of reduced pilE expression in the carriage isolates, especially NZCM238. Threshold cycle $\left(\Delta C_{T}\right)$ is presented in the graph, with higher $\Delta C_{T}$ corresponding to lower expression. There is no detectable expression in NZCM238 $(n=6), 71 \%$ $\pm 17.5 \%$ (SD, $n=6)$ reduction in NZCM239, and $94 \% \pm 3 \%(S D, n=6)$ reduction in NZCM240, relative to NZ97/192. (D) TNF- $\alpha$ secretion was significantly reduced when $16 \mathrm{HBE}$ cells were infected with NZCM238 or a pilE-pilS deletion mutant of NZ97/192 (NZ97192 1 pilE/S) compared with infection with wild-type NZ97/ $192(n=6)$. Error bars indicate standard deviations. ${ }^{*}, P<0.05 ;{ }^{* *}, P<0.001 ;{ }^{* * *}, P<0.0001$. (E) Electron micrographs of NZ97/192 (left), NZCM238 (center), and the NZ97/192 $\triangle$ pilE/S deletion strain. T4P are seen only with wild-type NZ97/192 (arrows).

$n=6$ ) reduction in NZCM240, all relative to NZ97/192 (Fig. 6C). T4P have been shown to induce TNF- $\alpha$ expression in endothelial cells (46); to investigate whether reduced pilE expression could explain the lower levels of TNF- $\alpha$ induction in epithelial cells, we constructed a pilin locus deletion. The entire pilE and pils antigenic variation region from NZ97/192 was replaced with an antibiotic resistance marker. Using a TNF- $\alpha$ enzyme-linked immunosorbent assay (ELISA), we demonstrated that the NZ97/192 $\triangle$ pilE/S mutant was not able to elicit TNF- $\alpha$ from epithelial cells, suggesting that intact pili play a role in inducing TNF- $\alpha$ from respiratory epithelial cells (Fig. 6D). These data suggest that one explanation for the low TNF- $\alpha$ levels elicited by NZCM238 (Fig. 2C) is that this isolate lacks T4P. The absence of detectable T4P in NZCM238 and the NZ97/192 $\triangle$ pilE/S mutant was confirmed by transmission electron microscopy (Fig. 6E).

We were unable to complement the NZ97/192 $\triangle$ pilE/S mutant, which could not be transformed. However, deletions in the pilE/S locus in two other isolates described 
elsewhere (NZ98/254, a New Zealand epidemic disease-associated isolate, and NZCM107, an unrelated serogroup B carriage isolate [47]) also resulted in reduced TNF- $\alpha$ secretion. Additionally, the carriage-associated isolate NZCM246, also predicted to have a large deletion within the pilE-pils region, confirmed by genomic PCR, showed a reduced ability to induce TNF- $\alpha$ relative to the household-associated disease isolate NZ98/058 (data not shown).

\section{DISCUSSION}

In this study, we demonstrated evidence of meningococcal transmission within households, using whole-genome sequencing of closely related household contact isolates. Isolates from the same household were more closely related to each other, as evident by clustering on maximum-likelihood phylogeny, than to isolates of the same sequence type from other households (Fig. 1). To understand whether these genomic differences could impact how isolates interact with the host, we chose one household and examined phenotypic and gene expression differences among the isolates. We observed phenotypic and transcriptional differences (Fig. 2 and 3). We also linked genomic differences to expression and phenotype differences, using mutagenesis to demonstrate that the presence of pili correlates with TNF- $\alpha$ induction by meningococci in respiratory epithelial cells (Fig. 6). Analyzing the genetic and phenotypic differences of closely related disease- and carriage-associated meningococci may shed light on virulence mechanisms. All isolates studied here were originally collected for analysis of potential vaccine antigens, including the serogroup and PorA type, and so only a single colony was retained for long-term storage in the reference library. Because $N$. meningitidis is so highly heterogeneous, this study likely only captures a snapshot of the true diversity of the bacteria in both carriage and disease. Furthermore, because all household contacts of disease patients would have been treated chemoprophylactically for the disease, it is impossible to conclude that none of the healthy household contact carriers would have gone on to develop the invasive disease.

A similar approach has recently been published, involving deep sequencing of throat and invasive isolates from the same patients (43). This study, like ours, also identified phase variation switches and DNA rearrangements occurring in the pilin locus, although these rearrangements resulted in antigenic variation rather than loss of pili. This study concluded that most of the genetic variations that the authors detected resulted from stochastic processes, and they did not find evidence for within-host selection of invasive strains. Similarly, we did not identify any genetic signatures associated with isolates from the blood or CSF versus those from the throat.

It has long been known that household crowding is a risk factor for meningococcal disease (16-18). Our phylogenetic analysis supports transmission within households, although it is difficult to ascertain which isolate was the index isolate and how the transmission proceeded. In the NZ96/294 family, it may be argued that acquisition of the carriage isolate (NZCM149) and onset of the disease in a second household member occurred very close in time, as there are only 12 variants between those two isolates. The isolates in some households had more variants than others (Table 2), but this diversity did not seem to relate to patient age, nor did it correlate with whether the household contained members carrying a different strain. For example, there are 209 differences among the isolates within the NZ97/019 household, where the index patient was 1 year old and the household contact also carried the same strain, while in the NZ97/021 household, where the index patient was 3 years old and 1 of 2 household members sampled was carrying $N$. lactamica, only 10 differences were found. In the $\mathrm{NZ97/019}$ isolate, we detected a likely recombination event, affecting a 5-kb region that likely originated from a related nasopharyngeal commensal species, $N$. bergeri, which could explain the large number of variants occurring in this household (23). We found that six genes in NZ97/019 expressed alleles associated with a carriage species. Interestingly, the recombination event was detected in only one invasive isolate, suggesting that the $N$. bergeri-derived alleles did not impact the virulence potential of the recipient isolate. All of the ST- 42 and ST-154 isolates in our study had identical mutS 
and mutL alleles, suggesting that the differences in levels of variation between households were not due to differing hypermutator phenotypes. Another possible cause of high divergence in some families could be the length of carriage period in the index patient. In general, the incubation period for meningococcal disease is thought to be between 1 and 10 days. However, longer periods of carriage before disease development presumably can lead to higher diversity between the patient isolate and isolates from healthy household members, due to stochastic, recombination, and DNA rearrangement events.

High numbers of SNPs were found within households in our study, in contrast with a recent study of methicillin-resistant Staphylococcus aureus (MRSA) (48). When multiple household isolates of MRSA were analyzed by whole-genome sequencing, the mean number of SNPs found varied between $17.6 \pm 35$ and $12 \pm 19$. The much higher variability of $N$. meningitidis may be due to the natural competence of $N$. meningitidis and the documented ability of meningococci to take up DNA from the environment or from isolates during carriage $(25,26)$.

Many variants were found within mononucleotide runs and small-repeat regions among the household strains and were the result of slipped-strand mispairing, a major mechanism of phase variation of gene expression. Slipped-strand mispairing results in small heritable but reversible random changes that can result in "all-or-none" expression of regulated transcripts and proteins, when the homopolymeric tract occurs in the coding region, or more subtle modulations of gene expression, when the tract occurs in promoter regions (42). Genes that encode surface and exposed proteins of pathogenic bacteria often contain DNA repeats in intergenic or coding regions indicative of phase-variable gene regulation (43). The siaD gene, known to be phase variable, was found to be in an "off" state in three carriage-associated isolates, due to slipped-strand mispairing that resulted in a frameshift mutation; in contrast, siaD was predicted to be intact and expressed in all of the disease-associated isolates; these predictions were confirmed by slide agglutination tests (49). The presence of the capsule is one of the key features of invasive meningococcal strains. Changes in expression of other phasevariable genes were found in both disease- and carriage-associated isolates. The potential significance of these changes is difficult to assess. By comparing genome sequence and transcriptome expression, we have potentially identified two transcripts under phase-variable control, NMBNZ0533_0037 (a putative lipoprotein) and NMBNZ0533_0093 (a conserved hypothetical protein). The NMBNZ0533_0037 transcript is predicted to encode a protein that is located in the membrane or secreted, although the location of the predicted protein encoded by NMBNZ0533_0093 could not be determined.

In addition to surface molecules, predicted to interact with the host, our transcriptome analysis highlights the possible importance of metabolic differences in invasive disease development, in particular the sulfate assimilation pathway. Seven of the 37 most differentially expressed genes in the NZ97/192 household were predicted to participate in the sulfate assimilation pathway, which catalyzes the metabolism and uptake of sulfate from the environment. Assimilated sulfate is used to generate multiple important molecules, including cysteine, methionine, iron-sulfur proteins, and other sulfur-containing macromolecules. Several studies have shown the importance of sulfur-containing compounds for detoxification in Salmonella, Bacillus, and Staphylococcus aureus (50-52). Cysteine and sulfite reductases have been shown to be important for Bordetella pertussis and Brucella melitensis virulence $(53,54)$. In Mycobacterium tuberculosis, mutants in the pathway have attenuated virulence in mice (55). Little is known about the sulfate assimilation pathway in $N$. meningitidis, which efficiently acquires environmental sulfate and can grow on a variety of sulfur sources (56). It has been suggested that sulfite reduction is important for nasopharyngeal colonization, as genome comparisons have shown that both $N$. meningitidis and $N$. lactamica, which specialize in nasopharyngeal colonization, have intact cysl-cysG operons, while Neisseria gonorrhoeae does not (33). Our data suggest an additional role for the sulfate assimilation pathway in development of invasive disease. A recent study has shown 
that the sulfate assimilation pathway is upregulated when meningococci are cultured under low-cysteine conditions and under stress; similarly, several studies have found genes encoding sulfate transporter permeases to be among the first genes to be expressed upon adherence to the epithelium $(13,15,57,58)$. The CSF is a relatively nutrient-poor medium and is thought to have to low cysteine levels, although signature-tagged mutagenesis studies have revealed that sulfur acquisition genes are essential during growth on minimal medium but are dispensable in serum, suggesting that cysteine can be acquired from serum $(59,60)$. Invasive bacteria are likely to encounter reactive oxygen species, generated by immune cells. The generation of glutathione, via the cysteine biosynthesis pathway, is one means of protection of the cells from oxidative stress (44). Our data confirmed that higher intracellular glutathione levels, following oxidative stress, were found in an isolate with higher expression of sulfate assimilation enzymes, and elevated glutathione could be detected even after overnight incubation without oxidative stress. Although we did not detect any differences in the susceptibilities of the various isolates to oxidative stress, the impact of various levels of intracellular glutathione may be apparent under other conditions of stress. Our quantitative PCR analyses revealed that the expression of $s b p, c y s N$, and cys $G$ genes was more variable than the transcriptome sequencing (RNA-seq) analysis suggested; this may be due to the sensitivity of metabolic pathways to minor variations in culture conditions. Meningococcal sulfate assimilation enzymes, encoded by genes cysl, cysJ, cys $N$, cys $D$, and $c y s H$, have high sequence identity to these enzymes in other bacteria. As for other bacteria, the genomic organization of genes encoding these enzymes suggests that they may be coregulated, as they are colinear with little spacing between each open reading frame. Our transcriptome analysis demonstrated that expression of these genes differed in similar direction and magnitude, a finding that supports coregulation. However, we did not identify putative transcription factors for their regulation. The role of sulfate metabolism in $N$. meningitidis carriage and disease is a subject of ongoing and future research.

T4P are an important virulence factor in N. meningitidis, participating in colonization and disease. Pili are major external structures that are exposed to the host immune system and therefore undergo extensive antigenic variation. Two classes of T4P have been described in meningococci. The New Zealand epidemic strain has class 1 pili, which are structurally similar to pili expressed in the related pathogen $N$. gonorrhoeae. The gonococcus has been shown to vary the amino acid composition of the major pilus protein, pilin (pilE), by a process of gene conversion $(61,62)$. The pilE locus can recombine with fragments of pilin coding sequence in the pils region to alter the amino acid composition of the final protein. Variation of the expressed pilin gene and rearrangement of the pilin antigenic region have been demonstrated in vivo, in volunteer studies of $N$. gonorrhoeae infection, and, for $N$. meningitidis, during an accidental human passage and through comparison of throat and invasive isolates from the same patients $(43,63-65)$. Our study confirmed that pilin variation frequently occurs during carriage as well, as evidenced by the variations in the pilin antigenic region among multiple carriage isolates. We found that the degree of variation in the pils region differed between household isolates. Within some household groups, there were almost no differences between disease- and carriage-associated isolates, while in others large deletions were found in carriage-associated isolates compared to their respective index disease isolate (e.g., NZCM238 and NZCM246). Genomic and transcriptome data suggest that NZCM238 lacks pili, an observation that was supported by electron microscopy (Fig. 6E) as well as our observation that NZCM238 could not be naturally transformed. Although pili have been frequently considered essential for colonization, it has been reported that $N$. meningitidis strains isolated from the nasopharynx are often nonpiliated, relying on alternative adhesins; under these circumstances, the opacity proteins (Opa and Opc) can mediate adhesion (66). Indeed, we did observe that the opacity proteins were expressed in our isolates and that there were not large differences in adherence to epithelial cells (data not shown and Fig. 2). In contrast, T4P have been shown to be essential during invasive infection of the host by 
meningococci. Specifically, type IV pili were shown to be essential to adhere to human endothelial cells lining dermal vessels, in a humanized mouse model, which had grafted human skin tissue (67). T4P also have been shown to induce microvasculature lesions and inflammation, which are responsible for the clinical symptoms of fulminant meningococcal disease (68). T4P have been shown to bind to CD147, a receptor of the immunoglobulin (lg) superfamily, which is expressed at high levels on endothelial cells of the brain microvasculature (69). Although CD147 is also expressed in epithelial cells, additional mechanisms of adherence apparently enable nonpiliated carriage strains to persist in the host nasopharyngeal mucosa.

Using mutagenesis, we demonstrated that type $\mathrm{VI}$ pili are associated with the induction of TNF- $\alpha$ from respiratory epithelial cells. A similar observation has previously been made in endothelial cells, although these cells secreted TNF- $\alpha$ only when monocytic cells were also present (46). In contrast, no human TNF- $\alpha$ was detected during infection in a humanized mouse model (a SCID mouse with a human skin graft), suggesting that the transplanted vasculature, including endothelial and skin cells, is not the source of systemic TNF- $\alpha$ during meningococcal disease. Human interleukin-6 (IL-6) and IL-8, in contrast, were detected in this model system (67). A study in N. gonorrhoeae found that removal of the pili resulted in loss of TNF- $\alpha$ secretion by epithelial cells, although this was seen only for bacteria also lacking opacity proteins $\left(\mathrm{Opa}^{-}\right)$and hence was likely due to the reduced adherence of the Pil-Opa- strain (70). However, our data suggest that the loss of TNF- $\alpha$ secretion, in our model system, is independent of adherence. Although NZCM238 was found to lack pili and did not elicit TNF- $\alpha$ secretion from $16 \mathrm{HBE}$ cells, this isolate adhered to $16 \mathrm{HBE}$ cells as well as, or better than, related piliated isolates. The differences reported in TNF- $\alpha$ secretion from epithelial cells could be due to the different bacterial species and host cell types tested.

Our findings suggest that pili are dispensable during meningococcal carriage and that they may even be detrimental to colonizing bacteria in the nasopharynx, as their presence could potentially trigger a localized proinflammatory response by the host, resulting in an influx of neutrophils and removal before transmission to a new host can occur. Whether meningococci actively alter the host immune response to facilitate long-term carriage, through either suppression of local cytokine expression or other means, remains an unanswered question. Also unknown is whether local proinflammatory cytokine production plays a role in the development of invasive disease, the process by which virulent strains access deeper tissues following a breach of the epithelial layer in the throat.

The fact that the T4P of Neisseria meningitidis are dispensable for colonization of mucosal surfaces highlights a key difference between $N$. meningitidis and the related pathogen $N$. gonorrhoeae, which uses pili to adhere to mucosal surfaces, where it elicits a strong local inflammatory response. This response leads to an influx of neutrophils, which do not control the infection; viable gonococci have frequently been observed inside neutrophils, where they delay fusion with granules, interfere with the respiratory burst, and delay apoptosis (71-74). The means by which carriage $N$. meningitidis suppresses a local inflammatory response, allowing it to persist in a healthy host for long periods, remain unknown. Although tissue culture cells only recapitulate some of the interactions between $N$. meningitidis and the host, they are a useful tool, as $N$. meningitidis is human restricted, and animal models for nasopharyngeal colonization have not yet been developed. The interaction is likely to be influenced by other cell types, including innate immune cells and other microbes of the normal microflora; much remains to be discovered about the complex interaction between host and microbe.

\section{MATERIALS AND METHODS}

Bacterial strains and growth conditions. N. meningitidis isolates were collected as described during a household contact study carried out in Auckland in the late 1990s (12). Isolates were maintained by the Meningococcal Reference Laboratory (MRL) at the Institute of Environmental Science and Research (ESR), as part of the surveillance of meningococcal disease in New Zealand on behalf of the Ministry of Health. The carriage isolates described in the present study (designated NZCM) were collected from healthy 
patient contacts by peroral nasopharyngeal swab (12). Although three colonies were collected from each individual, where possible, the studies described here were performed on bacteria derived from single colonies that were stored at $-80^{\circ} \mathrm{C}$ long-term (12). Isolates from the index patient were cultured from blood or CSF samples. All isolates were routinely typed using serological and sequencing methods to determine serogroup and serosubtype (por $A$ allele); in addition, selected strains were further analyzed, with the serotype (porB allele) determined by serology and sequence type (ST) determined by multilocus sequence typing (MLST) as described elsewhere (75-77). Serogrouping was typically carried out using slide agglutination; where these results were ambiguous, or where the isolate was autoagglutinating, PCR was used to determine serogroup. The subtype was determined by whole-cell enzyme immunoassay; isolates that were not subtypeable were subjected to porA-PCR analysis. Slide agglutination was also carried out for isolates in this study that had undergone phase variation of the siaD gene, using antiserum to the serogroup B New Zealand epidemic strain type, a gift from ESR. All isolates in the MRL were immediately frozen in Trypticase soy broth with $15 \%$ glycerol at $-80^{\circ} \mathrm{C}$ following minimal laboratory passage. Frozen working stocks of frequently accessed isolates are maintained to prevent repeated freeze-thaw cycles. $N$. meningitidis was grown on Columbia blood agar (CBA) plates (Fort Richard Laboratories, Auckland, New Zealand), for routine passage, or on BBL brain heart infusion (BHI) agar (Oxoid) plates, supplemented with kanamycin $(50 \mu \mathrm{g} / \mathrm{ml})$ where required, at $36^{\circ} \mathrm{C}$ in a humidified $5 \% \mathrm{CO}_{2}$ incubator. Escherichia coli strain DH5 $\alpha$ (subcloning efficiency) or Top10 cells (both from Life Technologies) were used for DNA manipulation and were grown on Luria-Bertani agar plates supplemented with kanamycin $(50 \mu \mathrm{g} / \mathrm{ml})$ or ampicillin $(100 \mu \mathrm{g} / \mathrm{ml})$.

Genomic DNA purification. Genomic DNA was purified from meningococci with the Gentra Puregene Yeast/Bact. kit (Qiagen, USA) according to the manufacturer's instructions, with the following changes. Bacteria, grown on CBA plates overnight, were scraped from the plate $(\sim 10 \mu \mathrm{l})$, resuspended in $300 \mu \mathrm{l}$ of lysis buffer, and incubated at $56^{\circ} \mathrm{C}$ for $1 \mathrm{~h}$ to kill meningococci, after which the remaining steps were followed. DNA concentration and quality were assessed by electrophoresis, 260/280 ratio, and the Quant-iT PicoGreen double-stranded DNA (dsDNA) assay kit (Thermo Fisher, USA).

Whole-genome sequencing, assembly, and variation analysis. Isolates were sequenced by New Zealand Genomics Limited (NZGL). NZ97/192 and NZCM238 samples were prepared using the Nextera DNA library format, and NZCM239 and NZCM240 samples were prepared using the TruSeq library format, while all other isolates were prepared with TruSeq Nano libraries (lllumina, USA). All samples were sequenced by Illumina MiSeq using paired-end sequencing, 2 by 150 bp for NZ97/192 and NZCM238 and 2 by $250 \mathrm{bp}$ for all other isolates. Adaptor trimmed reads were quality trimmed using Trimmomatic- 0.32 at a Phred quality greater than 20 and a minimum length of $100 \mathrm{bp}$ (78). Paired reads were aligned to RefSeq genome NC_017518.1 (Neisseria meningitidis NZ-05/33), and variants, single nucleotide variants (SNPs) and small insertions and deletions (indels), were identified using Freebayes 1.0 and GATK 3.3.0 UnifiedGenotyper tool with ploidy set to $1(79,80)$. For Freebayes workflow, reads were aligned using Bowtie 2.1.0, with settings to allow for local alignments (options: -nondeterministic, -local) and converted to sorted and indexed BAM files using SAMtools-0.1.19-44428cd (options: - $-\mathrm{b}-\mathrm{q} 20)(81,82)$. For GATK workflow, reads were aligned using bwa mem and converted using Picard-tools-1.119 (Broad Institute). GATK variants were filtered by GATK hardfilter setting. Freebayes variants were filtered by vcffilter within the vcflib toolkit (83). Variants were kept if they were found by both Freebayes and UnifiedGenotyper and had a quality of $>30$, an alternate frequency of $>70 \%$, a depth of $>10$, and at least 1 alternate read in forward and reverse direction. Variant annotation and effects were predicted by SnpEff (19). Common variations among groups of isolates were obtained using the isec function in bcftools (84). Select variants were confirmed by PCR and Sanger sequencing. De novo assemblies were carried out using SPAdes 3.5.0 (85), and contigs greater than 500 bp were kept and annotated with Prokka 1.12 (86). Functional change prediction was determined using the PROVEAN protein webtool (http://provean.jcvi.org/seq_submit .php). Predicted amino acid variants were compared to the NZ-05/33 reference protein sequence and were determined to be deleterious if the PROVEAN score was less than -2.5 .

Analysis of relatedness among household isolates. Using the published New Zealand epidemic strain type (RefSeq genome NC_017518.1; N. meningitidis NZ-05/33, New Zealand epidemic strain type, 2005) as a reference genome, we identified genetic differences (deletions [dels], indels, and SNPs) between the isolates and the reference strain $(87,88)$. Core SNPs were identified using Snippy and Snippy-core (v 3.1) (87). Maximum-likelihood phylogeny was generated using PhyML with the HKY substitution model with bootstrapping $(200 \times)(89,90)$. To investigate the origin of a highly variable $5-k b$ region, the draft genome from isolate NZ97/019 was aligned to the reference genome, NZ-05/33, and the genomic region corresponding to NZ-05/33 bp 185000 to 190142 was extracted and searched against the Neisseria multilocus sequence typing website (https:/pubmlst.org/neisseria/) (22). The resulting alleles were then used to identify the species of isolates that they were associated with.

Cell culture and infection. Bronchial respiratory epithelial cells (16HBE140-, abbreviated as $16 \mathrm{HBE}$ [27]) were routinely cultured in M199 medium supplemented with $10 \%$ inactivated fetal calf serum (FCS). For infection experiments, $16 \mathrm{HBE}$ cells were suspended at $6 \times 10^{4} \mathrm{cells} / \mathrm{ml}$ and cultured in 6 - or 24 -well plates until confluent. Prior to initiating infection, cells were washed with phosphate-buffered saline (PBS) and incubated in serum-free M199 for 16 to $24 \mathrm{~h}$.

Enumeration of cell-associated and intracellular bacteria. The number of $16 \mathrm{HBE}$ cell-associated bacteria (i.e., adherent and intracellular) was quantified using a method previously described (47). Briefly, confluent, serum-starved cells in 6-well plates were infected with $\mathrm{N}$. meningitidis at a multiplicity of infection (MOI) of $\sim 10$. After $4 \mathrm{~h}$, cells were washed three times with warm PBS to remove non-cellassociated bacteria and lysed with $1 \%$ saponin in PBS. Dilutions of the lysate were plated out; input bacteria were also enumerated by plating a series of dilutions. To determine the number of intracellular 
bacteria, the same method was used, but $100 \mu \mathrm{g} / \mathrm{ml}$ gentamicin was added for $1 \mathrm{~h}$ and then removed by washing prior to addition of saponin, to kill extracellular bacteria. All isolates were assayed in parallel with three replicates per isolate. Experiments were performed at least twice. Statistical significance was tested using an unpaired $t$ test.

TNF- $\alpha$ secretion from 16HBE cells. Confluent, serum-starved 16HBE cells in 24-well plates were infected with $N$. meningitidis at an $\mathrm{MOI}$ of $\sim 10$. After $24 \mathrm{~h}$, cell culture medium was collected and centrifuged to remove bacteria. Aliquots of cleared medium were stored at $-80^{\circ} \mathrm{C}$ until ready for use. A commercially available ELISA kit was used to determine TNF- $\alpha$ levels, according to the manufacturer's instructions (R\&D Systems, USA). All isolates were assayed in parallel with three replicates per isolate. Experiments were performed at least twice. Statistical significance was tested using an unpaired $t$ test.

RNA-seq sample preparation. Total RNA was isolated from mid-log-phase $N$. meningitidis bacteria that were exposed to $16 \mathrm{HBE}$ cells. $N$. meningitidis was scraped from fresh plates and resuspended at an optical density at $600 \mathrm{~nm}\left(\mathrm{OD}_{600}\right)$ of 0.25 in M199 medium supplemented with $10 \% \mathrm{FCS}$. This bacterial suspension ( $1 \mathrm{ml}$ ) was added to 24 -well plates with confluent $16 \mathrm{HBE}$ cells, to activate expression of genes induced by host cell factors. After $2 \mathrm{~h}$, the bacterial suspension $(900 \mu \mathrm{l})$ was combined with $100 \mu \mathrm{l}$ of stop solution, $5 \%$ buffer-equilibrated phenol $(\mathrm{pH} 7.4)$ in ethanol. Total RNA was then purified essentially as described by Gaynor et al. (91). Briefly, after the bacterial suspension was combined with stop solution, the cells were collected by centrifugation for $5 \mathrm{~min}$ at room temperature. The pellets were frozen at $-80^{\circ} \mathrm{C}$ until needed and then briefly thawed at room temperature and resuspended in $50 \mu \mathrm{l}$ of 0.4- $\mathrm{mg} / \mathrm{ml}$ lysozyme (Sigma) in $10 \mathrm{mM}$ Tris, pH 8.0, $1 \mathrm{mM}$ EDTA, and incubated for $5 \mathrm{~min}$. Cells were lysed by adding $950 \mu \mathrm{l}$ of Trizol reagent (Thermo Fisher, USA) and vortexing, followed by the addition of $200 \mu \mathrm{l}$ of chloroform. After centrifugation at $14,000 \times g$ for $15 \mathrm{~min}$, the top phase was combined, in a new tube, with an equivalent volume of $70 \%$ ethanol. This solution was then applied to an RNeasy Mini column (Qiagen) and washed according to the manufacturer's instructions. Samples were treated with an on-column RNase-free DNase kit (Qiagen). RNA concentration and quality were assessed with a 2100 Bioanalyzer instrument (Agilent Technologies); only samples with an RNA integrity number of $\geq 8$ were used for subsequent steps. rRNA was removed using a MICROBExpress bacterial mRNA enrichment kit (Thermo Fisher, USA), according to the manufacturer's instructions. The Bioanalyzer was used again to check the RNA following the mRNA enrichment step. Double-stranded cDNA was generated from $3 \mu \mathrm{g}$ of enriched bacterial mRNA using the SuperScript double-stranded cDNA synthesis kit (Thermo Fisher, USA). The quantity of cDNA was determined with the Quant-iT PicoGreen dsDNA assay kit (Thermo Fisher, USA), while the quality was checked by PCR of a housekeeping meningococcal gene (aroE). Double-stranded CDNA ( $\geq 700 \mathrm{ng} /$ sample) was sequenced by Ambry Genetics (CA, USA). TruSeq 2- by 100-bp paired-end libraries were generated, indexed, and sequenced on a HiSeq lane, producing 26 GB of compressed sequence data.

Analysis of transcriptome sequencing data. Bowtie2 version 2.1.0 (82) was used for mapping quality-trimmed sequences to targets. A Bowtie 2 index was built for Silva large-subunit and smallsubunit rRNA sequences (version r111). This index was used to prefilter reads with Bowtie 2 to exclude rRNA sequences. The NZ-05/33 genome was downloaded from NCBI (http://www.ncbi.nlm.nih.gov/ nuccore/385856165) and extended by 209 bp to the end of the sequence to account for circular sequence. The NZ-05/33 transcriptome GTF file was downloaded from Ensembl (http://bacteria.ensembl .org/info/data/ftp/index.html) (92), converted to gff3 format, and altered to make identifiers (IDs) unique. Transcripts were extracted and converted to FASTA format using a custom script, extending $51 \mathrm{bp}$ from the gene limits. A local Bowtie2 mapping (-k 2 --local --no-unal -no-mixed) was carried out for non-rRNA sequence against the extended $N$. meningitidis NZ-05/33 genome for all samples. Raw counts for each Bowtie/transcript mapping were generated using SAMtools. DESeq (31) was used to normalize the count data and produce differential expression probabilities, using an "all-versus-all" comparison of each isolate against each other isolate. Two sets of differentially expressed genes (for any comparison) were produced: a strict set with a DESeq adjusted probability of $<0.05$ and a relaxed set with either a DESeq adjusted probability of $<0.1$ or a fold change of $>2 \times$. DESeq was used to create a variance-stabilized transformation of the transcript counts, and heat maps of differentially expressed genes were created from these normalized expression values. Gene ontology assessments were performed by UniProt (32). Predicted subcellular localization was determined using PSORTb analysis, and functional predictions were checked with NeMeSys (https://www.genoscope.cns.fr/agc/microscope/collabprojects/nemesys .php) $(33,34)$. The Venn diagram was generated with BioVenn (93).

Analysis of transcript variants. The raw fastq files from RNA-seq were cleaned for low-quality segments, stretches of N's, and inclusion of adaptor sequence. Overlapping read pairs were collapsed into single reads, using AdapterRemoval, v. 1.5.4 (94). The cleaned-up reads were then mapped to the reference genome (http://www.ncbi.nlm.nih.gov/nuccore/CP002424) using Bowtie2 with default parameters (82). The BAM files were processed using SAMtools (-q $25-Q 0)$ to generate mpileup output (81). This output was used to create high-confidence genotypes for each strain using SNPest v. 1.0 (minimum depth of 10 reads, minimum posterior probability of 0.999 , minimum support for indel of $90 \%$ ) (95). The reference EMBL file was processed using an in-house script to extract all coding sequence (CDS) regions, including protein ID, start and end coordinates, strand, DNA sequence, and the encoded protein sequence. A custom program that reads the list of CDS regions and a list of high-quality genotypes (SNPs and indels) was implemented. If one or more predicted mutations were in a CDS, the corresponding DNA sequence was changed, and the mutated protein sequence was generated. A new FASTA file was generated for each strain containing every mutated protein relative to the reference. These files were then compared to the reference proteins. Orthologous proteins were identified by identifying the best reciprocal matches in a pairwise proteome comparison, performed with phmmer from the HMMER3.0 
package, and then the bit scores for individual domains in NZ97/192 were subtracted from the NZCM238 scores to produce a delta-bit score (DBS) (96-98). Genes with DBS values in the top $1 \%$ were flagged as potentially functionally important and further inspected for mapping error.

Sanger sequencing of selected variants. Selected variants were confirmed by PCR and Sanger sequencing. PCR assays were carried out with platinum Taq high-fidelity DNA polymerase (Thermo Fisher, USA) and purified genomic DNA. Primers are listed in Table S4 in the supplemental material.

qRT-PCR. Quantitative reverse transcriptase PCR (qRT-PCR) primers and probes were designed with the RealTimeDesign software (Biosearch Technologies, Inc.) and were generated by Life Technologies. Real-time primers were labeled at the 5' end with 6-FAM (6-fluorescein amidite) fluorescent dye and were coupled with BHQ-1 quencher (black hole quencher 1). PCR specificity was determined by agarose gel electrophoresis and sequencing of products. For simplicity, all PCR assays were carried out using $250 \mathrm{nM}$ probe and $200 \mathrm{nM}$ primer (Table S3), using TaqMan universal master mix II with uracil- $\mathrm{N}$-glycosylase (UNG; Thermo Fisher, USA). The following cycling program was used: $52^{\circ} \mathrm{C}$ for $2 \mathrm{~min}$ ( 1 cycle), $95^{\circ} \mathrm{C}$ for $10 \mathrm{~min}$ ( $1 \mathrm{cycle}$ ), and $95^{\circ} \mathrm{C}$ for $10 \mathrm{~s}$ and $58^{\circ} \mathrm{C}$ for $1 \mathrm{~min}$ ( $\left.45 \mathrm{cycles}\right)$. Reaction efficiency was determined using a standard curve with different concentrations of genomic DNA. The meningococcal genes aroE and ctrA were used as controls as RNA-seq analysis indicated that their expression did not significantly differ among the isolates. All assays were performed in parallel with three technical replicates per sample. Statistical significance was determined using an unpaired $t$ test.

Hydrogen peroxide sensitivity assay and measurement of intracellular glutathione levels. Fresh cultures of $N$. meningitidis were scraped from CBA plates and resuspended in brain heart infusion $(\mathrm{BHI})$ broth (Oxoid). Bacteria were incubated at $37^{\circ} \mathrm{C}$ in a $5 \% \mathrm{CO}_{2}$ incubator until the $\mathrm{OD}_{600}$ reached 0.6 . Cultures were split into three aliquots of $1 \mathrm{ml}$ each. Hydrogen peroxide (Sigma-Aldrich) was added to two of the cultures, at 2 and $5 \mathrm{mM}$ final concentrations, while the third culture was not exposed. Cultures were incubated at $37^{\circ} \mathrm{C}$ in a $5 \% \mathrm{CO}_{2}$ incubator for $20 \mathrm{~min}$. To remove hydrogen peroxide, the bacteria were pelleted at $400 \times g$ for $10 \mathrm{~min}$. The supernatant was discarded, and the pellets were resuspended in $1 \mathrm{ml} \mathrm{BHI}$ broth. Viable bacteria were enumerated by plating dilutions onto CBA plates. Following overnight growth, bacteria were scraped from the CBA plates (separate from those used to count colonies) and resuspended in PBS at an $\mathrm{OD}_{600}$ of 1.0. Bacteria were pelleted by centrifugation at $1,610 \times$ $g$ for $10 \mathrm{~min}$, and the supernatant was discarded. The bacterial pellet was deproteinized by suspension in 3 volumes of $5 \%$-sulfosalicylic acid solution and then lysed with two freeze-thaw cycles, alternating between liquid nitrogen and a $37^{\circ} \mathrm{C}$ water bath. Samples were placed at $4^{\circ} \mathrm{C}$ for 5 min and then centrifuged for $10 \mathrm{~min}$ at top speed in a microcentrifuge. Glutathione concentrations were assayed using a glutathione assay kit (Sigma-Aldrich). Experiments were performed three times, in triplicate.

Analysis of pilin locus. The pilus genomic region from NZ97/192, encoding NMBNZ0533_0019 (the major pilin PilE) and the silent PilS antigenic variation region, was deleted and replaced by the AphA3 kanamycin resistance cassette by natural recombination, essentially as described previously but in a different host isolate (47). PCR of the pilE/pils locus was carried out using primers pilEF1-HindIII and pilER1-BamHI (47).

Electron microscopy of Neisseria meningitidis. Bacteria and type 4 pili were visualized by transmission electron microscopy. Fresh cultures of bacteria on CBA plates were scraped into a $2 \%$ glutaraldehyde solution in PBS. The bacteria were then processed for negative staining essentially as described by Burghardt and Droleskey (99) and imaged at the Otago Centre for Electron Microscopy.

Accession number(s). The whole-genome sequencing and transcriptome sequencing reads are available through the EMBL Nucleotide Sequence Database (ENA; https://www.ebi.ac.uk/ena) under study accession numbers PRJEB23076 and PRJEB23077, respectively.

SUPPLEMENTAL MATERIAL

Supplemental material for this article may be found at https://doi.org/10.1128/

mSystems.00127-17.

TABLE S1, XLSX file, $0.1 \mathrm{MB}$.

TABLE S2, XLSX file, $0.1 \mathrm{MB}$.

TABLE S3, DOCX file, $0.1 \mathrm{MB}$.

TABLE S4, DOCX file, $0.02 \mathrm{MB}$.

\section{REFERENCES}

1. Stephens DS, Greenwood B, Brandtzaeg P. 2007. Epidemic meningitis, meningococcaemia, and Neisseria meningitidis. Lancet 369:2196-2210. https://doi.org/10.1016/50140-6736(07)61016-2.

2. Caugant DA, Froholm LO, Bovre K, Holten E, Frasch CE, Mocca LF, Zollinger WD, Selander RK. 1986. Intercontinental spread of a genetically distinctive complex of clones of Neisseria meningitidis causing epidemic disease. Proc Natl Acad Sci U S A 83:4927-4931. https://doi.org/10.1073/ pnas.83.13.4927.

3. Caugant DA, Mocca LF, Frasch CE, Froholm LO, Zollinger WD, Selander RK 1987. Genetic structure of Neisseria meningitidis populations in relation to serogroup, serotype, and outer membrane protein pattern. J Bacterio 169:2781-2792. https://doi.org/10.1128/jb.169.6.2781-2792.1987.
4. Dyet K, Devoy A, McDowell R, Martin D. 2005. New Zealand's epidemic of meningococcal disease described using molecular analysis: implications for vaccine delivery. Vaccine 23:2228-2230. https://doi.org/10 .1016/j.vaccine.2005.01.050.

5. Dyet KH, Martin DR. 2006. Clonal analysis of the serogroup B meningococci causing New Zealand's epidemic. Epidemiol Infect 134:377-383. https://doi.org/10.1017/S0950268805004954.

6. Devoy AF, Dyet KH, Martin DR. 2005. Stability of PorA during a meningococcal disease epidemic. J Clin Microbiol 43:832-837. https://doi.org/ 10.1128/JCM.43.2.832-837.2005.

7. O'Hallahan J, Lennon D, Oster P, Lane R, Reid S, Mulholland K, Stewart J, Penney L, Percival T, Martin D. 2005. From secondary prevention to 
primary prevention: a unique strategy that gives hope to a country ravaged by meningococcal disease. Vaccine 23:2197-2201. https://doi .org/10.1016/j.vaccine.2005.01.061.

8. Oster P, Lennon D, O'Hallahan J, Mulholland K, Reid S, Martin D. 2005. MeNZB: a safe and highly immunogenic tailor-made vaccine against the New Zealand Neisseria meningitidis serogroup B disease epidemic strain. Vaccine 23:2191-2196. https://doi.org/10.1016/j.vaccine.2005.01.063.

9. Kelly C, Arnold R, Galloway Y, O'Hallahan J. 2007. A prospective study of the effectiveness of the New Zealand meningococcal B vaccine. Am J Epidemiol 166:817-823. https://doi.org/10.1093/aje/kwm147.

10. Loring BJ, Turner N, Petousis-Harris H. 2008. MeNZB vaccine and epidemic control: when do you stop vaccinating? Vaccine 26:5899-5904. https://doi.org/10.1016/j.vaccine.2008.08.062.

11. Lopez L, Sherwood J. 2014. The epidemiology of meningococcal disease in New Zealand in 2013. Institute of Environmental Science and Research (ESR), Wellington, New Zealand.

12. Simmons G, Martin D, Stewart J, Jones N, Calder L, Bremner D. 2001. Carriage of Neisseria meningitidis among household contacts of patients with meningococcal disease in New Zealand. Eur J Clin Microbiol Infect Dis 20:237-242. https://doi.org/10.1007/PL00011260.

13. Joseph B, Schneiker-Bekel S, Schramm-Glück A, Blom J, Claus H, Linke B, Schwarz RF, Becker A, Goesmann A, Frosch M, Schoen C. 2010. Comparative genome biology of a serogroup $\mathrm{B}$ carriage and disease strain supports a polygenic nature of meningococcal virulence. J Bacteriol 192:5363-5377. https://doi.org/10.1128/JB.00883-10.

14. Stephens DS. 2009. Biology and pathogenesis of the evolutionarily successful, obligate human bacterium Neisseria meningitidis. Vaccine 27(Suppl 2):B71-B77. https://doi.org/10.1016/j.vaccine.2009.04.070.

15. Grifantini R, Bartolini E, Muzzi A, Draghi M, Frigimelica E, Berger J, Ratti G, Petracca R, Galli G, Agnusdei M, Giuliani MM, Santini L, Brunelli B, Tettelin H, Rappuoli R, Randazzo F, Grandi G. 2002. Previously unrecognized vaccine candidates against group $B$ meningococcus identified by DNA microarrays. Nat Biotechnol 20:914-921. https://doi.org/10.1038/ nbt728.

16. Olcén P, Kjellander J, Danielsson D, Lindquist BL. 1981. Epidemiology of Neisseria meningitidis; prevalence and symptoms from the upper respiratory tract in family members to patients with meningococcal disease. Scand J Infect Dis 13:105-109. https://doi.org/10.3109/inf.1981.13.issue -2.05 .

17. Cooke RP, Riordan T, Jones DM, Painter MJ. 1989. Secondary cases of meningococcal infection among close family and household contacts in England and Wales, 1984-7. BMJ 298:555-558. https:/doi.org/10.1136/ bmj.298.6673.555.

18. Baker M, McNicholas A, Garrett N, Jones N, Stewart J, Koberstein V, Lennon D. 2000. Household crowding a major risk factor for epidemic meningococcal disease in Auckland children. Pediatr Infect Dis J 19: 983-990. https://doi.org/10.1097/00006454-200010000-00009.

19. Cingolani P, Platts A, Wang LL, Coon M, Nguyen T, Wang L, Land SJ, Lu $X$, Ruden DM. 2012. A program for annotating and predicting the effects of single nucleotide polymorphisms, SnpEff. Fly 6:80-92. https://doi.org/ 10.4161/fly.19695.

20. Choi Y, Sims GE, Murphy S, Miller JR, Chan AP. 2012. Predicting the functional effect of amino acid substitutions and indels. PLoS One 7:e46688. https://doi.org/10.1371/journal.pone.0046688.

21. Choi Y, Chan AP. 2015. PROVEAN web server: a tool to predict the functional effect of amino acid substitutions and indels. Bioinformatics 31:2745-2747. https://doi.org/10.1093/bioinformatics/btv195.

22. Jolley KA, Maiden MC. 2010. BIGSdb: scalable analysis of bacterial genome variation at the population level. BMC Bioinformatics 11:595. https://doi.org/10.1186/1471-2105-11-595.

23. Bennett JS, Jolley KA, Earle SG, Corton C, Bentley SD, Parkhill J, Maiden MC. 2012. A genomic approach to bacterial taxonomy: an examination and proposed reclassification of species within the genus Neisseria. Microbiology 158:1570-1580. https://doi.org/10.1099/mic.0.056077-0.

24. Berger U. 1985. First isolation of Neisseria polysacchareae species nova in the Federal Republic of Germany. Eur J Clin Microbiol 4:431-433. https:// doi.org/10.1007/BF02148705.

25. Beddek AJ, Li MS, Kroll JS, Jordan TW, Martin DR. 2009. Evidence for capsule switching between carried and disease-causing Neisseria meningitidis strains. Infect Immun 77:2989-2994. https://doi.org/10.1128/IAI $.00181-09$.

26. Caugant DA, Bøvre K, Gaustad P, Bryn K, Holten E, Høiby EA, Frøholm LO. 1986. Multilocus genotypes determined by enzyme electrophoresis of

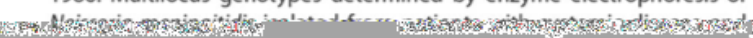
msystems.asmorg 20 November/December 2017 - Volume 2 issue 6 reo 127 ij from healthy carriers. J Gen Microbiol 132:641-652. https://doi.org/10 1099/00221287-132-3-641.

27. Cozens AL, Yezzi MJ, Kunzelmann K, Ohrui T, Chin L, Eng K, Finkbeiner WE, Widdicombe JH, Gruenert DC. 1994. CFTR expression and chloride secretion in polarized immortal human bronchial epithelial cells. Am J Respir Cell Mol Biol 10:38-47. https://doi.org/10.1165/ajrcmb.10.1 .7507342 .

28. Waage A, Halstensen A, Espevik T. 1987. Association between tumour necrosis factor in serum and fatal outcome in patients with meningococcal disease. Lancet i:355-357. https://doi.org/10.1016/50140-6736(87) 91728-4.

29. Waage A, Halstensen A, Shalaby R, Brandtzaeg P, Kierulf P, Espevik T. 1989. Local production of tumor necrosis factor alpha, interleukin 1, and interleukin 6 in meningococcal meningitis. Relation to the inflammatory response. J Exp Med 170:1859-1867. https://doi.org/10.1084/jem.170.6 .1859.

30. Ramsey KH, Schneider H, Cross AS, Boslego JW, Hoover DL, Staley TL, Kuschner RA, Deal CD. 1995. Inflammatory cytokines produced in response to experimental human gonorrhea. J Infect Dis 172:186-191. https://doi.org/10.1093/infdis/172.1.186.

31. Anders S, Huber W. 2010. Differential expression analysis for sequence count data. Genome Biol 11:R106. https://doi.org/10.1186/gb-2010-11 $-10-r 106$

32. UniProt Consortium. 2015. UniProt: a hub for protein information. Nucleic Acids Res 43:D204-D212. https://doi.org/10.1093/nar/gku989.

33. Rusniok C, Vallenet D, Floquet S, Ewles $\mathrm{H}$, Mouzé-Soulama C, Brown D, Lajus A, Buchrieser C, Médigue C, Glaser P, Pelicic V. 2009. NeMeSys: a biological resource for narrowing the gap between sequence and function in the human pathogen Neisseria meningitidis. Genome Biol 10: R110. https://doi.org/10.1186/gb-2009-10-10-r110.

34. Yu NY, Wagner JR, Laird MR, Melli G, Rey S, Lo R, Dao P, Sahinalp SC, Ester M, Foster U, Brinkman FS. 2010. PSORTb 3.0: improved protein subcelIular localization prediction with refined localization subcategories and predictive capabilities for all prokaryotes. Bioinformatics 26:1608-1615. https://doi.org/10.1093/bioinformatics/btq249.

35. Virji M, Makepeace K, Moxon ER. 1994. Distinct mechanisms of interactions of Opc-expressing meningococci at apical and basolateral surfaces of human endothelial cells; the role of integrins in apical interactions. Mol Microbiol 14:173-184. https://doi.org/10.1111/j.1365-2958.1994.tb01277.x.

36. Virji M, Makepeace K, Peak IRA, Ferguson DJP, Jennings MP, Moxon ER. 1995. Opc- and pilus-dependent interactions of meningococci with human endothelial cells: molecular mechanisms and modulation by surface polysaccharides. Mol Microbiol 18:741-754. https://doi.org/10 .1111/j.1365-2958.1995.mmi_18040741.x.

37. Unkmeir A, Latsch K, Dietrich G, Wintermeyer E, Schinke B, Schwender S, Kim KS, Eigenthaler M, Frosch M. 2002. Fibronectin mediates Opcdependent internalization of Neisseria meningitidis in human brain microvascular endothelial cells. Mol Microbiol 46:933-946. https://doi.org/ 10.1046/j.1365-2958.2002.03222.x.

38. de Vries FP, Cole R, Dankert J, Frosch M, van Putten JP. 1998. Neisseria meningitidis producing the Opc adhesin binds epithelial cell proteoglycan receptors. Mol Microbiol 27:1203-1212. https://doi.org/10.1046/j $.1365-2958.1998 .00763 . x$.

39. Massari P, Ho Y, Wetzler LM. 2000. Neisseria meningitidis porin PorB interacts with mitochondria and protects cells from apoptosis. Proc Natl Acad Sci U S A 97:9070-9075. https://doi.org/10.1073/pnas.97.16.9070.

40. Massari P, King CA, Ho AY, Wetzler LM. 2003. Neisserial PorB is translocated to the mitochondria of HeLa cells infected with Neisseria meningitidis and protects cells from apoptosis. Cell Microbiol 5:99-109. https:// doi.org/10.1046/j.1462-5822.2003.00257.x.

41. Massari P, Gunawardana J, Liu X, Wetzler LM. 2010. Meningococcal porin PorB prevents cellular apoptosis in a toll-like receptor 2 - and NFkappaB-independent manner. Infect Immun 78:994-1003. https://doi .org/10.1128/IAI.00156-09.

42. Siena E, D'Aurizio R, Riley D, Tettelin H, Guidotti S, Torricelli G, Moxon ER, Medini D. 2016. In-silico prediction and deep-DNA sequencing validation indicate phase variation in 115 Neisseria meningitidis genes. BMC Genomics 17:843. https://doi.org/10.1186/s12864-016-3185-1.

43. Klughammer J, Dittrich $M$, Blom J, Mitesser V, Vogel U, Frosch $M$, Goesmann A, Müller T, Schoen C. 2017. Comparative genome sequencing reveals within-host genetic changes in Neisseria meningitidis during invasive disease. PLoS One 12:e0169892. https://doi.org/10.1371/journal .pone. 0169892 . 
lence in Neisseria meningitidis. Front Cell Infect Microbiol 4:114. https:// doi.org/10.3389/fcimb.2014.00114.

45. Carmel-Harel O, Storz G. 2000. Roles of the glutathione- and thioredoxindependent reduction systems in the Escherichia coli and Saccharomyces cerevisiae responses to oxidative stress. Annu Rev Microbiol 54: 439-461. https://doi.org/10.1146/annurev.micro.54.1.439.

46. Taha MK. 2000. Neisseria meningitidis induces the expression of the TNF-alpha gene in endothelial cells. Cytokine 12:21-25. https://doi.org/ 10.1006/cyto.1999.0506.

47. Ren X, MacKichan JK. 2014. Disease-associated Neisseria meningitidis isolates inhibit wound repair in respiratory epithelial cells in a type IV pilus-independent manner. Infect Immun 82:5023-5034. https://doi.org/ 10.1128/IAI.02001-14.

48. Alam MT, Read TD, Petit RA, III, Boyle-Vavra S, Miller LG, Eells SJ, Daum RS, David MZ. 2015. Transmission and microevolution of USA300 MRSA in U.S. households: evidence from whole-genome sequencing. mBio 6:e00054-15. https://doi.org/10.1128/mBio.00054-15.

49. Hammerschmidt $S$, Müller $A$, Sillmann $H$, Mühlenhoff $M$, Borrow R, Fox $A$, van Putten J, Zollinger WD, Gerardy-Schahn R, Frosch M. 1996. Capsule phase variation in Neisseria meningitidis serogroup B by slipped-strand mispairing in the polysialyltransferase gene (siaD): correlation with bacterial invasion and the outbreak of meningococcal disease. Mol Microbiol 20:1211-1220. https://doi.org/10.1111/j.1365-2958.1996.tb02641.x

50. Turnbull AL, Surette MG. 2008. L-Cysteine is required for induced antibiotic resistance in actively swarming Salmonella enterica serova Typhimurium. Microbiology 154:3410-3419. https://doi.org/10.1099/ mic. $0.2008 / 020347-0$.

51. Hullo MF, Martin-Verstraete I, Soutourina O. 2010. Complex phenotypes of a mutant inactivated for CymR, the global regulator of cysteine metabolism in Bacillus subtilis. FEMS Microbiol Lett 309:201-207. https:// doi.org/10.1111/j.1574-6968.2010.02043.x.

52. Soutourina O, Dubrac S, Poupel O, Msadek T, Martin-Verstraete I. 2010. The pleiotropic CymR regulator of Staphylococcus aureus plays an important role in virulence and stress response. PLoS Pathog 6:e1000894. https://doi.org/10.1371/journal.ppat.1000894.

53. Bogdan JA, Nazario-Larrieu J, Sarwar J, Alexander P, Blake MS, 2001. Bordetella pertussis autoregulates pertussis toxin production through the metabolism of cysteine. Infect Immun 69:6823-6830. https://doi.org/10 .1128/IAl.69.11.6823-6830.2001.

54. Lestrate P, Delrue RM, Danese I, Didembourg C, Taminiau B, Mertens P, De Bolle X, Tibor A, Tang CM, Letesson JJ. 2000. Identification and characterization of in vivo attenuated mutants of Brucella melitensis. Mo Microbiol 38:543-551. https://doi.org/10.1046/j.1365-2958.2000.02150.x.

55. Senaratne RH, De Silva AD, Williams SJ, Mougous JD, Reader JR, Zhang T, Chan S, Sidders B, Lee DH, Chan J, Bertozzi CR, Riley LW. 2006. 5'-Adenosinephosphosulphate reductase (CysH) protects Mycobacterium tuberculosis against free radicals during chronic infection phase in mice. Mol Microbiol 59:1744-1753. https://doi.org/10.1111/j.1365-2958 2006.05075.x.

56. Port JL, DeVoe IW, Archibald FS. 1984. Sulphur acquisition by Neisseric meningitidis. Can J Microbiol 30:1453-1457. https://doi.org/10.1139/m84 -232 .

57. van de Waterbeemd B, Zomer G, van den ljssel J, van Keulen L, Eppink MH, van der Ley P, van der Pol LA. 2013. Cysteine depletion causes oxidative stress and triggers outer membrane vesicle release by Neisseric meningitidis; implications for vaccine development. PLoS One 8:e54314. https://doi.org/10.1371/journal.pone.0054314.

58. Dietrich G, Kurz S, Hübner C, Aepinus C, Theiss S, Guckenberger M, Panzner U, Weber J, Frosch M. 2003. Transcriptome analysis of Neisseria meningitidis during infection. J Bacteriol 185:155-164. https://doi.org/10 1128/JB.185.1.155-164.2003.

59. Wishart DS, Jewison T, Guo AC, Wilson M, Knox C, Liu Y, Djoumbou Y, Mandal R, Aziat F, Dong E, Bouatra S, Sinelnikov I, Arndt D, Xia J, Liu P, Yallou F, Bjorndahl T, Perez-Pineiro R, Eisner R, Allen F, Neveu V, Greiner R, Scalbert A. 2013. HMDB 3.0-the Human Metabolome Database in 2013. Nucleic Acids Res 41:D801-D807. https://doi.org/10.1093/nar/ gks1065.

60. Mendum TA, Newcombe J, Mannan AA, Kierzek AM, McFadden J. 2011. Interrogation of global mutagenesis data with a genome scale model of Neisseria meningitidis to assess gene fitness in vitro and in sera. Genome Biol 12:R127. https://doi.org/10.1186/gb-2011-12-12-r127.

61. Segal E, Billyard E, So M, Storzbach S, Meyer TF. 1985. Role of chromosomal rearrangement in $N$. gonorrhoeae pilus phase variation. Cell 40: 293-300. https://doi.org/10.1016/0092-8674(85)90143-6.
62. Swanson J, Bergström S, Robbins K, Barrera O, Corwin D, Koomey JM. 1986. Gene conversion involving the pilin structural gene correlates with pilus ${ }^{+}$in equilibrium with pilus ${ }^{-}$changes in Neisseria gonorrhoeae. Cell 47:267-276. https://doi.org/10.1016/0092-8674(86)90449-6.

63. Seifert HS, Wright CJ, Jerse AE, Cohen MS, Cannon JG. 1994. Multiple gonococcal pilin antigenic variants are produced during experimental human infections. J Clin Invest 93:2744-2749. https://doi.org/10.1172/ JCI117290.

64. Swanson J, Robbins K, Barrera O, Corwin D, Boslego J, Ciak J, Blake M, Koomey JM. 1987. Gonococcal pilin variants in experimental gonorrhea. J Exp Med 165:1344-1357. https://doi.org/10.1084/jem.165.5.1344.

65. Omer H, Rose G, Jolley KA, Frapy E, Zahar JR, Maiden MC, Bentley SD, Tinsley CR, Nassif X, Bille E. 2011. Genotypic and phenotypic modifications of Neisseria meningitidis after an accidental human passage. PLoS One 6:e17145. https://doi.org/10.1371/journal.pone.0017145.

66. Virji M, Makepeace K, Ferguson DJ, Achtman M, Moxon ER. 1993. Meningococcal Opa and Opc proteins: their role in colonization and invasion of human epithelial and endothelial cells. Mol Microbiol 10: 499-510. https://doi.org/10.1111/1.1365-2958.1993.tb00922x.

67. Melican K, Michea Veloso P, Martin T, Bruneval P, Duménil G. 2013. Adhesion of Neisseria meningitidis to dermal vessels leads to local vascular damage and purpura in a humanized mouse model. PLoS Pathog 9:e1003139. https://doi.org/10.1371/journal.ppat.1003139.

68. Join-Lambert $O$, Lecuyer $H$, Miller F, Lelievre L, Jamet A, Furio L, Schmitt A, Pelissier P, Fraitag S, Coureuil M, Nassif X. 2013. Meningococcal interaction to microvasculature triggers the tissular lesions of purpura fulminans. J Infect Dis 208:1590-1597. https://doi.org/10.1093/infdis/ jit301.

69. Bernard SC, Simpson N, Join-Lambert O, Federici C, Laran-Chich MP, Maïssa N, Bouzinba-Ségard H, Morand PC, Chretien F, Taouji S, Chevet E, Janel S, Lafont F, Coureuil M, Segura A, Niedergang F, Marullo S, Couraud PO, Nassif X, Bourdoulous S. 2014. Pathogenic Neisseria meningitidis utilizes CD147 for vascular colonization. Nat Med 20:725-731. https:// doi.org/10.1038/nm.3563.

70. Naumann M, Wessler S, Bartsch C, Wieland B, Meyer TF. 1997. Neisseria gonorrhoeae epithelial cell interaction leads to the activation of the transcription factors nuclear factor kappaB and activator protein 1 and the induction of inflammatory cytokines. J Exp Med 186:247-258. https://doi.org/10.1084/jem.186.2.247.

71. Johnson MB, Criss AK. 2013. Neisseria gonorrhoeae phagosomes delay fusion with primary granules to enhance bacterial survival inside human neutrophils. Cell Microbiol 15:1323-1340. https:/doi.org/10.1111/cmi .12117.

72. Smirnov A, Daily KP, Criss AK. 2014. Assembly of NADPH oxidase in human neutrophils is modulated by the opacity-associated protein expression state of Neisseria gonorrhoeae. Infect Immun 82:1036-1044. https://doi.org/10.1128/1Al.00881-13.

73. Criss AK, Katz BZ, Seifert HS. 2009. Resistance of Neisseria gonorrhoeae to non-oxidative killing by adherent human polymorphonuclear leucocytes. Cell Microbiol 11:1074-1087. https://doi.org/10.1111/j.1462-5822 2009.01308.x.

74. Chen A, Seifert HS. 2011. Neisseria gonorrhoeae-mediated inhibition of apoptotic signaling in polymorphonuclear leukocytes. Infect Immun 79:4447-4458. https://doi.org/10.1128/lAl.01267-10.

75. Jolley KA, Kalmusova J, Feil EJ, Gupta S, Musilek M, Kriz P, Maiden MC 2000. Carried meningococci in the Czech Republic: a diverse recombining population. J Clin Microbiol 38:4492-4498. https://doi.org/10.1128/ JCM.40.9.3549-3550.2002.

76. Bygraves JA, Urwin R, Fox AJ, Gray SJ, Russell JE, Feavers IM, Maiden MC. 1999. Population genetic and evolutionary approaches to analysis of Neisseria meningitidis isolates belonging to the ET-5 complex. J Bacteriol 181:5551-5556.

77. Maiden MC, Bygraves JA, Feil E, Morelli G, Russell JE, Urwin R, Zhang Q, Zhou J, Zurth K, Caugant DA, Feavers IM, Achtman M, Spratt BG. 1998. Multilocus sequence typing: a portable approach to the identification of clones within populations of pathogenic microorganisms. Proc Natl Acad Sci U S A 95:3140-3145. https://doi.org/10.1073/pnas.95.6.3140.

78. Bolger AM, Lohse M, Usadel B. 2014. Trimmomatic: a flexible trimmer for Illumina sequence data. Bioinformatics 30:2114-2120. https://doi.org/10 .1093/bioinformatics/btu170.

79. Garrison E, Marth G. 2012. Haplotype-based variant detection from short-read sequencing. arXiv arXiv:1207.3907v2 [q-bio.GN]. https://arxiv .org/abs/1207.3907v2.

80. DePristo MA, Banks E, Poplin R, Garimella KV, Maguire JR, Hartl C, 
Philippakis AA, del Angel G, Rivas MA, Hanna M, McKenna A, Fennell TJ, Kernytsky AM, Sivachenko AY, Cibulskis K, Gabriel SB, Altshuler D, Daly MJ. 2011. A framework for variation discovery and genotyping using next-generation DNA sequencing data. Nat Genet 43:491-498. https:// doi.org/10.1038/ng.806.

81. Li H, Handsaker B, Wysoker A, Fennell T, Ruan J, Homer N, Marth G, Abecasis G, Durbin R, 1000 Genome Project Data Processing Subgroup. 2009. The sequence alignment/map format and SAMtools. Bioinformatics 25:2078-2079. https://doi.org/10.1093/bioinformatics/btp352.

82. Langmead B, Salzberg SL. 2012. Fast gapped-read alignment with Bowtie 2. Nat Methods 9:357-359. https://doi.org/10.1038/nmeth. 1923.

83. Garrison E. 2013. A simple C++ library for parsing and manipulating VCF files. https://github.com/ekg/vcflib.

84. Li H. 2011. A statistical framework for SNP calling, mutation discovery, association mapping and population genetical parameter estimation from sequencing data. Bioinformatics 27:2987-2993. https://doi.org/10 .1093/bioinformatics/btr509.

85. Bankevich A, Nurk S, Antipov D, Gurevich AA, Dvorkin M, Kulikov AS, Lesin VM, Nikolenko SI, Pham S, Prjibelski AD, Pyshkin AV, Sirotkin AV, Vyahhi N, Tesler G, Alekseyev MA, Pevzner PA. 2012. SPAdes: a new genome assembly algorithm and its applications to single-cell sequencing. J Comput Biol 19:455-477. https://doi.org/10.1089/cmb.2012.0021.

86. Seemann T. 2014. Prokka: rapid prokaryotic genome annotation. Bioinformatics 30:2068-2069. https://doi.org/10.1093/bioinformatics/btu153.

87. Seemann T. 2014. Snippy. Rapid haploid variant calling and core SNP phylogeny. https://github.com/tseemann/snippy.

88. Budroni S, Siena E, Dunning Hotopp JC, Seib KL, Serruto D, Nofroni C, Comanducci M, Riley DR, Daugherty SC, Angiuoli SV, Covacci A, Pizza M, Rappuoli R, Moxon ER, Tettelin H, Medini D. 2011. Neisseria meningitidis is structured in clades associated with restriction modification systems that modulate homologous recombination. Proc Natl Acad Sci U S A 108:4494-4499. https://doi.org/10.1073/pnas.1019751108.

89. Tamura K, Dudley J, Nei M, Kumar S. 2007. MEGA4: Molecular Evolutionary Genetics Analysis (MEGA) software version 4.0. Mol Biol Evol 24: 1596-1599. https://doi.org/10.1093/molbev/msm092.

90. Guindon S, Dufayard JF, Lefort V, Anisimova M, Hordijk W, Gascuel O. 2010. New algorithms and methods to estimate maximum-likelihood phylogenies: assessing the performance of PhyML 3.0. Syst Biol 59: 307-321. https://doi.org/10.1093/sysbio/syq010.
91. Gaynor EC, Cawthraw S, Manning G, MacKichan JK, Falkow S, Newell DG. 2004. The genome-sequenced variant of Campylobacter jejuni NCTC 11168 and the original clonal clinical isolate differ markedly in colonization, gene expression, and virulence-associated phenotypes. J Bacteriol 186:503-517. https://doi.org/10.1128/JB.186.2.503-517.2004.

92. Kersey PJ, Allen JE, Armean I, Boddu S, Bolt BJ, Carvalho-Silva D, Christensen M, Davis P, Falin U, Grabmueller C, Humphrey J, Kerhornou A, Khobova J, Aranganathan NK, Langridge N, Lowy E, McDowall MD, Maheswari U, Nuhn M, Ong CK, Overduin B, Paulini M, Pedro H, Perry E, Spudich G, Tapanari E, Walts B, Williams G, Tello-Ruiz M, Stein J, Wei S, Ware D, Bolser DM, Howe KL, Kulesha E, Lawson D, Maslen G, Staines DM. 2016. Ensembl Genomes 2016: more genomes, more complexity. Nucleic Acids Res 44:D574-D580. https://doi.org/10.1093/nar/gkv1209.

93. Hulsen T, de Vlieg J, Alkema W. 2008. BioVenn - a web application for the comparison and visualization of biological lists using areaproportional Venn diagrams. BMC Genomics 9:488. https://doi.org/10 1186/1471-2164-9-488.

94. Lindgreen S. 2012. AdapterRemoval: easy cleaning of next-generation sequencing reads. BMC Res Notes 5:337. https://doi.org/10.1186/1756 -0500-5-337.

95. Lindgreen S, Krogh A, Pedersen JS. 2014. SNPest: a probabilistic graphical model for estimating genotypes. BMC Res Notes 7:698. https://doi .org/10.1186/1756-0500-7-698.

96. Finn RD, Bateman A, Clements J, Coggill P, Eberhardt RY, Eddy SR, Heger A, Hetherington K, Holm L, Mistry J, Sonnhammer EL, Tate J, Punta M. 2014. Pfam: the protein families database. Nucleic Acids Res 42: D222-D230. https://doi.org/10.1093/nar/gkt1223.

97. Finn RD, Clements J, Eddy SR. 2011. HMMER web server: interactive sequence similarity searching. Nucleic Acids Res 39:W29-W37. https:// doi.org/10.1093/nar/gkr367.

98. Wheeler NE, Barquist L, Kingsley RA, Gardner PP. 2016. A profile-based method for identifying functional divergence of orthologous genes in bacterial genomes. Bioinformatics 32:3566-3574. https://doi.org/10 .1093/bioinformatics/btw518.

99. Burghardt RC, Droleskey R. 2006. Transmission electron microscopy. Curr Protoc Microbiol Chapter 2:Unit 2B.1. https://doi.org/10.1002/978047 1729259.mc02b01s03. 